Supporting Information for:

\title{
Insertion of Alkylidene Carbenes into B-H Bonds
}

Ji-Min Yang ${ }^{\#}$, Feng-Kai Guo\#, Yu-Tao Zhao, Qiao Zhang, Ming-Yao Huang,

Mao-Lin Li, Shou-Fei Zhu*, and Qi-Lin Zhou*

State Key Laboratory and Institute of Elemento-Organic Chemistry, College of

Chemistry, Nankai University, Tianjin 300071, China

Email:sfzhu@nankai.edu.cn; qlzhou@nankai.edu.cn

\# These authors contributed equally to this work.

CONTENTS:

1. General Information....................................................2

2. Preparation of Alkenyl Triflates .....................................................2

3. Typical Procedures of B-H Bond Insertion Reactions and Additional

Studies on Reaction Conditions............................................................21

4. Analytical Data of Insertion Products ............................................23

5. X-ray Diffraction Analysis of Product 5q ........................................34

6. Transformations of the Products ...................................................36

7. Kinetic Isotope Effect Experiments ...........................................42

8. Computational Methods ......................................................................44

9. NMR Spectra of New Compounds ...........................................67

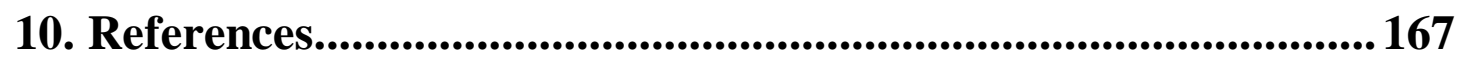




\section{General Information}

All reactions and manipulations were performed using standard Schlenk techniques. All solvents were purified and dried using standard procedures. ${ }^{1}$ Trimethylamineborane adduct was purchased form $J \& K$ and the other borane adducts were prepared according to the literature procedures. ${ }^{2}$ Melting points were measured on a RY-I apparatus and uncorrected. NMR spectra were recorded with a Bruker AV 400 spectrometer at $400 \mathrm{MHz}\left({ }^{1} \mathrm{H}\right.$ NMR), $101 \mathrm{MHz}\left({ }^{13} \mathrm{C}\right.$ NMR $), 128 \mathrm{MHz}\left({ }^{11} \mathrm{~B} \mathrm{NMR}\right)$. Chemical shifts (values) were reported in ppm down field from internal $\mathrm{Me}_{4} \mathrm{Si}\left({ }^{1} \mathrm{H}\right.$ and ${ }^{13} \mathrm{C}$ NMR). High Resolution Mass Spectra (HRMS) were recorded on an IonSpec FTICR mass spectrometer with Electron Spray Ionization (ESI) resource.

\section{Preparation of Alkenyl Triflates}

The alkenyl triflates 1 were prepared according to the published methods. ${ }^{3}$

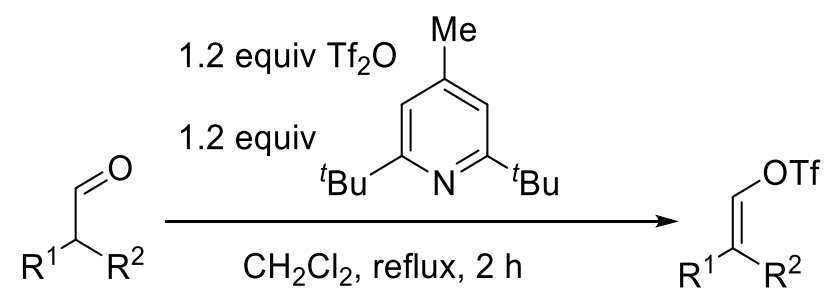

Typical procedure for synthesis of substrates 1 : Into a $250 \mathrm{~mL}$ round-bottom flask equipped with a reflux condenser and a stirrer, was added dry $\mathrm{CH}_{2} \mathrm{Cl}_{2}(100 \mathrm{~mL})$, aldehyde (30 mmol) and 2,6-di-tert-butyl-4-methylpyridine (7.4 g, $36 \mathrm{mmol}$ ) under an argon atmosphere. $\mathrm{Tf}_{2} \mathrm{O}(10.1 \mathrm{~g}, 36 \mathrm{mmol})$ was added dropwise and the mixture was stirred for $2 \mathrm{~h}$ at $50{ }^{\circ} \mathrm{C}$. Then the solvent was removed on evaporator and the residue combined with petroleum ether. The solid pyridinium triflate was filtered off and the filtrate was washed with $1 \mathrm{~N}$ hydrochloric acid $(100 \mathrm{~mL})$, brine $(50 \mathrm{~mL})$ and dried over $\mathrm{MgSO}_{4}$. After filtration and removal of volatiles, the residue was purified with silica gel chromatography using hexane as an eluent.

The following is the characterization data for the new compounds $\mathbf{1}$. 
(4-phenylcyclohexylidene)methyl trifluoromethanesulfonate (1a)

$\quad 61 \%$ yield. Colorless oil. ${ }^{1} \mathrm{H}$ NMR $\left(400 \mathrm{MHz}, \mathrm{CDCl}_{3}\right) \delta 7.30(\mathrm{t}, J$
$=7.4 \mathrm{~Hz}, 2 \mathrm{H}), 7.25-7.16(\mathrm{~m}, 3 \mathrm{H}), 6.45(\mathrm{~s}, 1 \mathrm{H}), 2.94(\mathrm{~d}, J=13.4$ $\mathrm{Hz}, 1 \mathrm{H}), 2.71(\mathrm{t}, J=12.1 \mathrm{~Hz}, 1 \mathrm{H}), 2.34(\mathrm{~d}, J=13.7 \mathrm{~Hz}, 1 \mathrm{H}), 2.16$ (t, $J=13.6 \mathrm{~Hz}, 1 \mathrm{H})$, $2.10-1.91(\mathrm{~m}, 3 \mathrm{H}), 1.60-1.48(\mathrm{~m}, 2 \mathrm{H}) .{ }^{13} \mathrm{C} \mathrm{NMR}\left(101 \mathrm{MHz}, \mathrm{CDCl}_{3}\right) \delta 145.68,132.45$, 128.51, 128.07, 126.72, 126.38, 118.70 (q, $J=322.2 \mathrm{~Hz}$ ), 43.87, 34.68, 33.61, 29.75, 25.96. HRMS (ESI) Calcd for $\left[\mathrm{C}_{14} \mathrm{H}_{15} \mathrm{~F}_{3} \mathrm{O}_{3} \mathrm{SNa}, \mathrm{M}+\mathrm{Na}\right]^{+}$: 343.0586; Found: 343.0604 .

\section{2-hexyloct-1-en-1-yl trifluoromethanesulfonate (1g)}

OTf $\quad 57 \%$ yield. Colorless oil. ${ }^{1} \mathrm{H}$ NMR $\left(400 \mathrm{MHz}, \mathrm{CDCl}_{3}\right) \delta 6.39(\mathrm{~s}, 1 \mathrm{H})$, ${ }^{n} \mathrm{C}_{6} \mathrm{H}_{13}{ }_{{ }^{n} \mathrm{C}_{6} \mathrm{H}_{13}} 2.14(\mathrm{dd}, J=8.9,6.5 \mathrm{~Hz}, 2 \mathrm{H}), 2.00(\mathrm{td}, J=7.5,1.3 \mathrm{~Hz}, 2 \mathrm{H}), 1.46-$ $1.35(\mathrm{~m}, 4 \mathrm{H}), 1.34-1.23(\mathrm{~m}, 12 \mathrm{H}), 0.97-0.79(\mathrm{~m}, 6 \mathrm{H}) .{ }^{13} \mathrm{C} \mathrm{NMR}$ $\left(101 \mathrm{MHz}, \mathrm{CDCl}_{3}\right) \delta 133.62,130.74,118.65(\mathrm{q}, J=322.2 \mathrm{~Hz}), 31.55,31.53,30.79$, 29.05, 28.76, 27.27, 27.13, 27.03, 22.55, 22.54, 13.97. HRMS (ESI) Calcd for $\left[\mathrm{C}_{15} \mathrm{H}_{31} \mathrm{~F}_{3} \mathrm{NO}_{3} \mathrm{~S}, \mathrm{M}+\mathrm{NH}_{4}\right]^{+}:$362.1971; Found: 362.3237.

3-(4-(tert-butyl)phenyl)-2-methylprop-1-en-1-yl trifluoromethanesulfonate (1h)

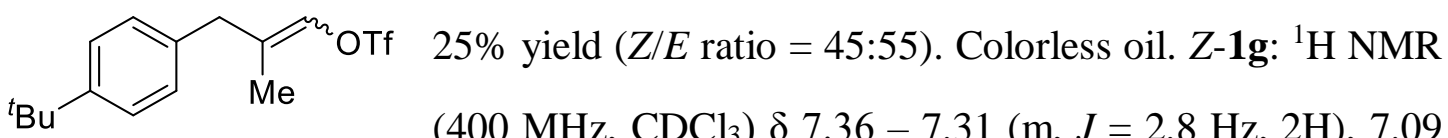
$(\mathrm{d}, J=2.1 \mathrm{~Hz}, 2 \mathrm{H}), 6.52-6.49(\mathrm{~m}, 1 \mathrm{H}), 3.46(\mathrm{~s}, 2 \mathrm{H}), 1.69(\mathrm{~d}, J=1.5 \mathrm{~Hz}, 3 \mathrm{H}), 1.31(\mathrm{~s}$, 9H). ${ }^{13} \mathrm{C}$ NMR $\left(101 \mathrm{MHz}, \mathrm{CDCl}_{3}\right) \delta 149.75,134.11,130.57,128.65,128.54,125.71$, $118.81(\mathrm{q}, J=322.2 \mathrm{~Hz}), 35.08,34.55,31.47,16.90 . E-1 \mathrm{~g}:{ }^{1} \mathrm{H} \mathrm{NMR}\left(400 \mathrm{MHz}, \mathrm{CDCl}_{3}\right)$ $\delta 7.36-7.31(\mathrm{~m}, J=2.8 \mathrm{~Hz}, 2 \mathrm{H}), 7.07(\mathrm{~d}, J=2.1 \mathrm{~Hz}, 2 \mathrm{H}), 6.54-6.52(\mathrm{~m}, 1 \mathrm{H}), 3.27$ (s, 2H), 1.69 (d, $J=1.5 \mathrm{~Hz}, 3 \mathrm{H}), 1.31$ (s, 9H). ${ }^{13} \mathrm{C}$ NMR (101 MHz, $\left.\mathrm{CDCl}_{3}\right) \delta$ 149.97, 134.03, 131.90, 129.78, 128.51, 125.71, 118.82 (q, $J=322.2 \mathrm{~Hz}), 39.09,34.58,31.47$, 13.82. The configuration of $\mathbf{1 g}$ was inferred by that of (3-(Benzyloxy)-2methylallyl)benzene $^{4}$ and 4,4,5,5-Tetramethyl-2-(2-methyl-3-phenylprop-1-en-1-yl)1,3,2-dioxaborolane ${ }^{5}$. HRMS (ESI) Calcd for $\left[\mathrm{C}_{15} \mathrm{H}_{23} \mathrm{~F}_{3} \mathrm{NO}_{3} \mathrm{~S}, \mathrm{M}+\mathrm{NH}_{4}\right]^{+}$: 354.1345; Found: 354.1338. 
The alkenyl triflates 4 were prepared according to the following procedure.

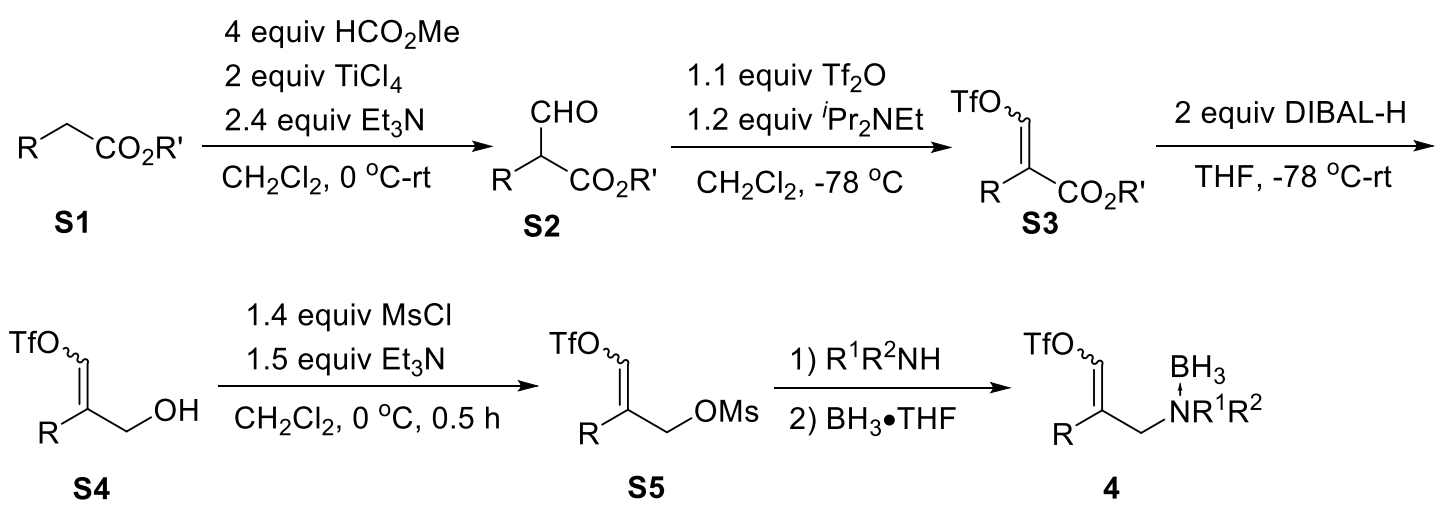

Under an argon atmosphere, ester $\mathbf{S 1}$ (11.5 mmol), methyl formate (2.76 g, $46.0 \mathrm{mmol})$ and dry $\mathrm{CH}_{2} \mathrm{Cl}_{2}(20 \mathrm{~mL})$ were introduced into a Schlenk flask. The solution was cooled to $0{ }^{\circ} \mathrm{C}$ and $\mathrm{TiCl}_{4}(4.32 \mathrm{~g}, 23.0 \mathrm{mmol})$ followed by triethylamine $(2.79 \mathrm{~g}, 27.6 \mathrm{mmol})$ were added dropwise through the syringe. The reaction mixture was stirred at $0{ }^{\circ} \mathrm{C}$ for $0.5 \mathrm{~h}$ and at room temperature for an extra hour. The mixture was poured onto cold water to quench the reaction and the aqueous layer was extracted with $\mathrm{CH}_{2} \mathrm{Cl}_{2}(3 \times 50$ $\mathrm{mL})$. The combined organic layer was washed with water, brine and dried over $\mathrm{Na}_{2} \mathrm{SO}_{4}$. Removal of solvent under vacuum afforded the formyl ester $\mathbf{S 2}$ that was carried to the next step without further purification.

To a solution of formyl ester $\mathbf{S 2}(11.5 \mathrm{mmol})$ in dry $\mathrm{CH}_{2} \mathrm{Cl}_{2}(15 \mathrm{~mL})$ was subsequently added ${ }^{i} \operatorname{Pr}_{2} \mathrm{NEt}(1.78 \mathrm{~g}, 13.8 \mathrm{mmol})$ and $\mathrm{Tf}_{2} \mathrm{O}(3.57 \mathrm{~g}, 12.7 \mathrm{mmol})$ at $-78{ }^{\circ} \mathrm{C}$ under a nitrogen atmosphere. The reaction mixture was stirred at $-78{ }^{\circ} \mathrm{C}$ for $30 \mathrm{~min}$. After warming to room temperature, the organic layer was concentrated under vacuum. The product was purified by silica gel column $(\mathrm{PE} / \mathrm{EA}=10: 1)$ to afford $\mathbf{S 3}$.

Under an argon atmosphere, $\mathbf{S 3}(5.0 \mathrm{mmol})$ and dry THF $(20 \mathrm{~mL})$ were introduced into a Schlenk flask. The solution was cooled to $-78{ }^{\circ} \mathrm{C}$ and DIBAL-H (10.0 mmol, $1.0 \mathrm{M}$ in hexanes) was added dropwise through the syringe. Then the reaction mixture was warmed to room temperature and stirred for $2 \mathrm{~h}$. The mixture was poured onto $2 \mathrm{~N}$ hydrochloric acid $(20 \mathrm{~mL})$ to quench the reaction and the aqueous layer was extracted with $\mathrm{Et}_{2} \mathrm{O}(3 \times 25 \mathrm{~mL})$. The combined organic layer was washed with water, brine and dried over $\mathrm{Na}_{2} \mathrm{SO}_{4}$. Removal of solvent under vacuum afforded the alcohol $\mathbf{S 4}$ that was carried to the next step without further purification. 
To a solution of the compound $\mathbf{S 4}(5.0 \mathrm{mmol})$ and $\mathrm{Et}_{3} \mathrm{~N}(759 \mathrm{mg}, 7.5 \mathrm{mmol})$ in $\mathrm{CH}_{2} \mathrm{Cl}_{2}$ $(150 \mathrm{~mL})$ at $0{ }^{\circ} \mathrm{C}$, methanesulfonyl chloride $(802 \mathrm{mg}, 7.0 \mathrm{mmol})$ was added dropwise. After stirring at $0{ }^{\circ} \mathrm{C}$ for $0.5 \mathrm{~h}$, saturated $\mathrm{NH}_{4} \mathrm{Cl}(50 \mathrm{~mL})$ was added, the two phases were separated, and the aqueous layer was extracted with $\mathrm{CH}_{2} \mathrm{Cl}_{2}(3 \times 50 \mathrm{~mL})$. The combined organic layer was washed with brine, dried over anhydrous $\mathrm{MgSO}_{4}$ and concentrated in vacuo to afford the product $\mathbf{S 5}$ which was pure enough for the next step. To a solution of the compound S5 $(5.0 \mathrm{mmol})$ in THF $(20 \mathrm{~mL}), \mathrm{R}^{1} \mathrm{R}^{2} \mathrm{NH}(20.0 \mathrm{mmol})$ was added, and the mixture was stirred at $45{ }^{\circ} \mathrm{C}$ for $12 \mathrm{~h}$. Then the reaction mixture was concentrated in vacuo, and filtered by column chromatography $(\mathrm{PE} / \mathrm{EA}=5: 1)$. Removal of solvent under vacuum afforded the amine that was carried to the next step without further purification.

To a solution of the amine $\mathbf{S 5}(1.0 \mathrm{mmol})$ in THF $(30 \mathrm{~mL}), \mathrm{BH}_{3} \cdot \mathrm{THF}(1.5 \mathrm{mmol}, 1.0$ $\mathrm{M}$ in THF) was added at $0{ }^{\circ} \mathrm{C}$, and the mixture was stirred at this temperature for $2 \mathrm{~h}$. Then evaporation and column chromatography $(\mathrm{PE} / \mathrm{EA}=15: 1)$ gave the desired product 4 .

The analytical data of the compounds S3 and $\mathbf{4}$ were listed as following.

\section{methyl-2-benzyl-3-(((trifluoromethyl)sulfonyl)oxy)acrylate (S3a)}

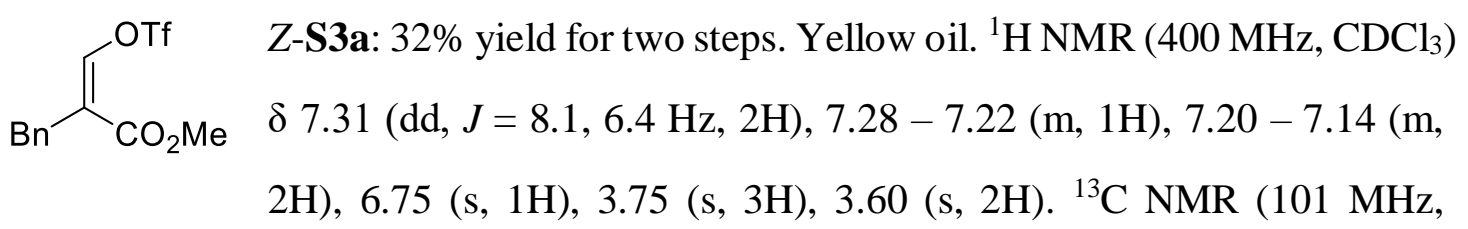
$\left.\mathrm{CDCl}_{3}\right) \delta 164.05,139.17,136.32,128.93,128.74,127.29,123.93,118.62(\mathrm{q}, J=322.2$ $\mathrm{Hz}), 52.47,35.25$.

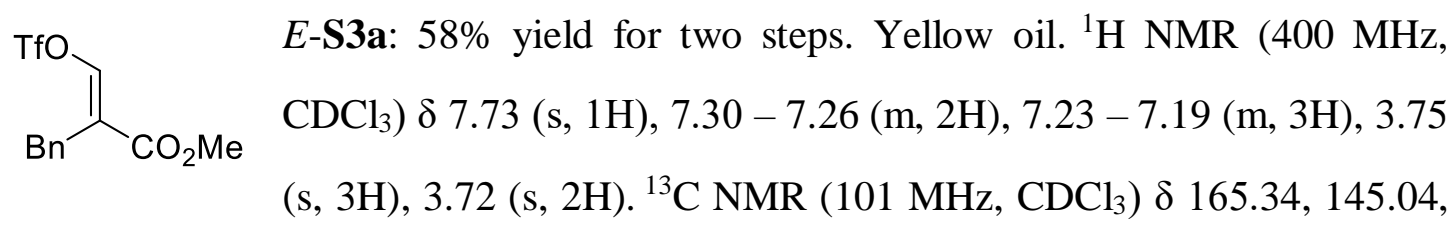
$137.27,128.76,128.61,126.90,122.86,118.53$ (q, $J=322.2 \mathrm{~Hz}), 52.56,30.55$. The configuration of (S3a) was confirmed by the NOESY NMR. HRMS (ESI) Calcd for $\left[\mathrm{C}_{12} \mathrm{H}_{11} \mathrm{~F}_{3} \mathrm{NaO}_{5} \mathrm{~S}, \mathrm{M}+\mathrm{Na}\right]^{+}:$347.0171; Found: 347.0175. 
methyl (E)-2-(4-chlorobenzyl)-3-(((trifluoromethyl)sulfonyl)oxy)acrylate (S3n)

$85 \%$ yield for two steps, only $(E)$-S3n was obtained. Yellow $\mathrm{CO}_{2} \mathrm{Me}$ oil. ${ }^{1} \mathrm{H}$ NMR $\left(400 \mathrm{MHz}, \mathrm{CDCl}_{3}\right) \delta 7.73(\mathrm{~s}, 1 \mathrm{H}), 7.25(\mathrm{~d}, J=$ $8.2 \mathrm{~Hz}, 2 \mathrm{H}), 7.15$ (d, $J=8.2 \mathrm{~Hz}, 2 \mathrm{H}), 3.77$ (s, 3H), 3.68 (s, 2H). ${ }^{13} \mathrm{C}$ NMR (101 MHz, $\left.\mathrm{CDCl}_{3}\right) \delta 165.07,145.13,135.64,132.69,129.88,128.78,122.26,118.39$ (q, $J=321.0$ $\mathrm{Hz}$ ), 52.56, 29.83. HRMS (ESI) Calcd for $\left[\mathrm{C}_{12} \mathrm{H}_{10} \mathrm{ClF}_{3} \mathrm{NaO}_{5} \mathrm{~S}, \mathrm{M}+\mathrm{Na}\right]^{+}$: 380.9782 ; Found: 380.9755.

methyl 2-(4-(trifluoromethyl)benzyl)-3-(((trifluoromethyl)sulfonyl)oxy)acrylate (S30)

83\% yield for two steps, $Z / E=51: 49$, the configuration of $\mathrm{CO}_{2} \mathrm{Me}$ S3o was inferred from S3a. Yellow oil. Z-S3o: ${ }^{1} \mathrm{H}$ NMR $\left(400 \mathrm{MHz}, \mathrm{CDCl}_{3}\right) \delta 7.59-7.54(\mathrm{~m}, 2 \mathrm{H}), 7.34-7.30(\mathrm{~m}, 2 \mathrm{H}), 6.87(\mathrm{~s}, 1 \mathrm{H}), 3.78(\mathrm{~s}$, 3H), $3.66(\mathrm{~s}, 2 \mathrm{H}) .{ }^{13} \mathrm{C} \mathrm{NMR}\left(101 \mathrm{MHz}, \mathrm{CDCl}_{3}\right) \delta 165.13,145.56,140.72,129.88$, 129.54, 129.04, 125.75 (q, $J=3.5 \mathrm{~Hz}), 122.83,118.53$ (q, $J=322.2 \mathrm{~Hz}), 52.66,35.11$. E-S3o: ${ }^{1} \mathrm{H}$ NMR (400 MHz, $\left.\mathrm{CDCl}_{3}\right) \delta 7.77(\mathrm{~s}, 1 \mathrm{H}), 7.59-7.54(\mathrm{~m}, 2 \mathrm{H}), 7.34-7.30$ (m, 2H), $3.78(\mathrm{~s}, 2 \mathrm{H}), 3.76(\mathrm{~s}, 3 \mathrm{H}) .{ }^{13} \mathrm{C} \mathrm{NMR}\left(101 \mathrm{MHz}, \mathrm{CDCl}_{3}\right) \delta$ 163.73, 141.36, $139.62,130.13,129.21,128.98,125.89$ (q, $J=3.5 \mathrm{~Hz}), 121.89,118.65$ (q, $J=322.2$ $\mathrm{Hz}$ ), 52.76, 30.34. HRMS (ESI) Calcd for $\left[\mathrm{C}_{13} \mathrm{H}_{10} \mathrm{~F}_{6} \mathrm{NaO}_{5} \mathrm{~S}, \mathrm{M}+\mathrm{Na}\right]^{+}: 415.0045$; Found: 415.0048.

methyl 2-(4-methoxybenzyl)-3-(((trifluoromethyl)sulfonyl)oxy)acrylate (S3p) $82 \%$ yield for two step, $Z / E=29: 71$, the configuration of $\mathrm{CO}_{2} \mathrm{Me}$ S3p was inferred from S3a. Yellow oil. Z-S3p: ${ }^{1} \mathrm{H}$ NMR $\left(400 \mathrm{MHz}, \mathrm{CDCl}_{3}\right) \delta 7.15-6.90(\mathrm{~m}, 2 \mathrm{H}), 6.85-6.80(\mathrm{~m}, 2 \mathrm{H}), 6.71(\mathrm{~s}, 1 \mathrm{H}), 3.79(\mathrm{~s}$, 3H), 3.76 (s, 3H), 3.54 (s, 2H). ${ }^{13} \mathrm{C} \mathrm{NMR}\left(101 \mathrm{MHz}, \mathrm{CDCl}_{3}\right) \delta 164.12,158.82,138.89$, 129.82, 128.11, 124.39, 118.61 (q, $J=322.2 \mathrm{~Hz}), 114.31,55.29,52.42,34.45 . E-\mathbf{S 3 p}$ : ${ }^{1} \mathrm{H}$ NMR (400 MHz, $\left.\mathrm{CDCl}_{3}\right) \delta 7.70(\mathrm{~s}, 1 \mathrm{H}), 7.15-6.90(\mathrm{~m}, 2 \mathrm{H}), 6.85-6.80(\mathrm{~m}, 2 \mathrm{H})$, $3.79(\mathrm{~s}, 3 \mathrm{H}), 3.76$ (s, 3H), 3.65 (s, 2H). ${ }^{13} \mathrm{C} \mathrm{NMR}\left(101 \mathrm{MHz}, \mathrm{CDCl}_{3}\right) \delta 165.36,158.56$, 144.71, 129.66, 129.24, 123.25, 118.51 (q, J = 322.2 Hz), 114.12, 55.29, 52.52, 29.68. 
HRMS (ESI) Calcd for $\left[\mathrm{C}_{13} \mathrm{H}_{13} \mathrm{~F}_{3} \mathrm{NaO}_{6} \mathrm{~S}, \mathrm{M}+\mathrm{Na}\right]^{+}$: 377.0277; Found: 377.0280 .

methyl 2-(4-fluorobenzyl)-3-(((trifluoromethyl)sulfonyl)oxy)acrylate (S3q)<smiles>CC(=O)C(=COc1ccc(F)cc1)Cc1ccccc1</smiles>
$86 \%$ yield for two steps, $Z / E=53: 47$, the configuration of $\mathbf{S 3 q}$ was inferred from S3a. Yellow oil. Z-S3q: ${ }^{1} \mathrm{H}$ NMR (400 MHz, $\left.\mathrm{CDCl}_{3}\right) \delta 7.20-7.13(\mathrm{~m}, 2 \mathrm{H}), 7.02-6.94(\mathrm{~m}, 2 \mathrm{H}), 6.77(\mathrm{~s}$, 1H), $3.76(\mathrm{~s}, 3 \mathrm{H}), 3.58(\mathrm{~s}, 2 \mathrm{H}) .{ }^{13} \mathrm{C} \mathrm{NMR}\left(101 \mathrm{MHz}, \mathrm{CDCl}_{3}\right) \delta 165.27,163.21(\mathrm{~d}, J=$ $21.6 \mathrm{~Hz}), 145.08,132.98(\mathrm{~d}, J=3.23 \mathrm{~Hz}), 130.30$ (d, $J=8.1 \mathrm{~Hz}), 122.74,118.63$ (q, $J$ $=322.2 \mathrm{~Hz}), 115.59(\mathrm{~d}, J=21.2 \mathrm{~Hz}), 52.54,34.55 . E-\mathbf{S 3 q}:{ }^{1} \mathrm{H} \mathrm{NMR}\left(400 \mathrm{MHz}, \mathrm{CDCl}_{3}\right)$ $\delta 7.72(\mathrm{~s}, 1 \mathrm{H}), 7.20-7.13(\mathrm{~m}, 2 \mathrm{H}), 7.02-6.94(\mathrm{~m}, 2 \mathrm{H}), 3.77(\mathrm{~s}, 3 \mathrm{H}), 3.69(\mathrm{~s}, 2 \mathrm{H}) .{ }^{13} \mathrm{C}$ $\operatorname{NMR}\left(101 \mathrm{MHz}, \mathrm{CDCl}_{3}\right) \delta 163.94,160.77$ (d, $\left.J=21.2 \mathrm{~Hz}\right), 139.16,132.07$ (d, $J=3.23$ $\mathrm{Hz}), 130.16(\mathrm{~d}, J=8.1 \mathrm{~Hz}), 123.78,118.52$ (q, $J=322.2 \mathrm{~Hz}), 115.83$ (d, $J=21.2 \mathrm{~Hz})$, 52.65, 29.80. HRMS (ESI) Calcd for $\left[\mathrm{C}_{12} \mathrm{H}_{10} \mathrm{~F}_{4} \mathrm{NaO}_{5} \mathrm{~S}, \mathrm{M}+\mathrm{Na}\right]^{+}: 365.0077$; Found: 365.0081 .

\section{methyl 2-methyl-3-(((trifluoromethyl)sulfonyl)oxy)acrylate (S3r)}

Me ${ }_{\mathrm{CO}_{2} \mathrm{Me}}^{73 \% \text { yield for two steps, } \mathrm{Z} / \mathrm{i}=88: 12 \text {, the configuration of } \mathbf{S 3 r} \text { was }}$ NMR (101 MHz, $\left.\mathrm{CDCl}_{3}\right) \delta 164.54,137.87,119.97,118.63$ (q, $J=322.2 \mathrm{~Hz}$ ), 52.47, 15.05. E-S3r: ${ }^{1} \mathrm{H}$ NMR (400 MHz, $\left.\mathrm{CDCl}_{3}\right) \delta 7.63(\mathrm{~d}, J=1.2 \mathrm{~Hz}, 1 \mathrm{H}), 3.80(\mathrm{~s}, 3 \mathrm{H}), 1.93$ $(\mathrm{d}, J=1.6 \mathrm{~Hz}, 3 \mathrm{H}) .{ }^{13} \mathrm{C} \mathrm{NMR}\left(101 \mathrm{MHz}, \mathrm{CDCl}_{3}\right) \delta 165.87,144.70,120.08,118.63$ (q, $J=322.2 \mathrm{~Hz}$ ), 52.56, 10.36. HRMS (ESI) Calcd for $\left[\mathrm{C}_{6} \mathrm{H}_{7} \mathrm{~F}_{3} \mathrm{NaO}_{5} \mathrm{~S}, \mathrm{M}+\mathrm{Na}\right]^{+}$: 270.9858; Found: 270.9863.

\section{methyl 2-((((trifluoromethyl)sulfonyl)oxy)methylene)hexanoate (S3s)}

$73 \%$ yield for two steps, $\mathrm{ZlE}=45: 55$, the configuration of $\mathbf{S 3 s}$ was
${ }^{n} \mathrm{Bu} \mathrm{CO}_{2} \mathrm{Me}$ inferred from S3a. Yellow oil. Z-S3s: ${ }^{1} \mathrm{H} \mathrm{NMR}\left(400 \mathrm{MHz}, \mathrm{CDCl}_{3}\right) \delta$ $6.73(\mathrm{~s}, 1 \mathrm{H}), 3.82(\mathrm{~s}, 3 \mathrm{H}), 2.33-2.24(\mathrm{~m}, 2 \mathrm{H}), 1.50-1.29(\mathrm{~m}, 4 \mathrm{H}), 0.92(\mathrm{t}, J=7.2 \mathrm{~Hz}$, $3 \mathrm{H}) .{ }^{13} \mathrm{C} \mathrm{NMR}\left(101 \mathrm{MHz}, \mathrm{CDCl}_{3}\right) \delta 164.63,137.46,124.68,118.64(\mathrm{q}, J=322.2 \mathrm{~Hz})$, 
52.42, 30.33, 29.22, 22.11, 13.79. E-S3s: ${ }^{1} \mathrm{H}$ NMR (400 MHz, $\left.\mathrm{CDCl}_{3}\right) \delta 7.63(\mathrm{~s}, 1 \mathrm{H})$, $3.80(\mathrm{~s}, 3 \mathrm{H}), 2.38(\mathrm{t}, J=8.0 \mathrm{~Hz}, 2 \mathrm{H}), 1.50-1.29(\mathrm{~m}, 4 \mathrm{H}), 0.92(\mathrm{t}, J=7.2 \mathrm{~Hz}, 3 \mathrm{H}) .{ }^{13} \mathrm{C}$ NMR (101 MHz, $\left.\mathrm{CDCl}_{3}\right) \delta 165.70,144.59,124.24,118.56$ (q, $\left.J=322.2 \mathrm{~Hz}\right), 52.46$, 30.36, 24.59, 22.43, 13.82. HRMS (ESI) Calcd for $\left[\mathrm{C}_{9} \mathrm{H}_{14} \mathrm{~F}_{3} \mathrm{O}_{5} \mathrm{~S}, \mathrm{M}+\mathrm{H}\right]^{+}: 291.0509$; Found: 291.0507.

\section{ethyl 3-methyl-2-((((trifluoromethyl)sulfonyl)oxy)methylene)butanoate (S3t)}

$\mathrm{TfO}_{2} \quad 73 \%$ yield for two steps, $Z / E=44: 56$, the configuration of S3t was ${ }^{i} \mathrm{Pr}{ }_{\mathrm{CO}_{2} \mathrm{Et}}$ inferred from S3a. Yellow oil. Z-S3t: ${ }^{1} \mathrm{H} \mathrm{NMR}\left(400 \mathrm{MHz}, \mathrm{CDCl}_{3}\right) \delta$ $6.63(\mathrm{~s}, 1 \mathrm{H}), 4.29(\mathrm{q}, J=8.0 \mathrm{~Hz}, 2 \mathrm{H}), 2.82-2.68(\mathrm{~m}, 1 \mathrm{H}), 1.32(\mathrm{t}, J=7.2,3 \mathrm{H}), 1.14$ $(\mathrm{d}, J=6.9 \mathrm{~Hz}, 6 \mathrm{H}) .{ }^{13} \mathrm{C} \mathrm{NMR}\left(101 \mathrm{MHz}, \mathrm{CDCl}_{3}\right) \delta 164.65,135.92,131.48,118.71$ (q, $J=322.2 \mathrm{~Hz}), 61.73,29.38,21.11,14.16 . E-S 3 t:{ }^{1} \mathrm{H}$ NMR $\left(400 \mathrm{MHz}, \mathrm{CDCl}_{3}\right) \delta 7.51$ $(\mathrm{s}, 1 \mathrm{H}), 4.24(\mathrm{q}, J=8.0 \mathrm{~Hz}, 2 \mathrm{H}), 2.98-3.13(\mathrm{~m}, 1 \mathrm{H}), 1.32(\mathrm{t}, J=7.2 \mathrm{~Hz}, 3 \mathrm{H}), 1.21(\mathrm{~d}$, $J=7.0 \mathrm{~Hz}, 6 \mathrm{H}) .{ }^{13} \mathrm{C} \mathrm{NMR}\left(101 \mathrm{MHz}, \mathrm{CDCl}_{3}\right) \delta 164.89,143.56,129.20,118.76$ (q, $J=$ 322.2 Hz), 61.44, 26.14, 20.33, 14.26. HRMS (ESI) Calcd for $\left[\mathrm{C}_{9} \mathrm{H}_{14} \mathrm{~F}_{3} \mathrm{O}_{5} \mathrm{~S}, \mathrm{M}+\mathrm{H}\right]^{+}$: 291.0509; Found: 291.0504.

\section{methyl 2-cyclohexyl-3-(((trifluoromethyl)sulfonyl)oxy)acrylate (S3u)}

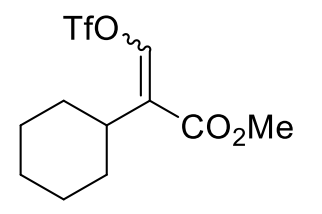

$89 \%$ yield for two steps, $Z / E=24: 76$, the configuration of $\mathbf{S 3} \mathbf{u}$ was inferred from S3a. Yellow oil. Z-S3u: ${ }^{1} \mathrm{H}$ NMR (400 MHz, $\left.\mathrm{CDCl}_{3}\right)$ $\delta 6.61(\mathrm{~s}, 1 \mathrm{H}), 3.82(\mathrm{~s}, 3 \mathrm{H}), 2.46-2.34(\mathrm{~m}, 1 \mathrm{H}), 1.90-1.52(\mathrm{~m}$, 7H), 1.35 - $1.14(\mathrm{~m}, 3 \mathrm{H}) .{ }^{13} \mathrm{C}$ NMR (101 MHz, $\left.\mathrm{CDCl}_{3}\right) \delta 165.21,136.38,130.62$, $118.62(\mathrm{q}, J=322.2 \mathrm{~Hz}), 52.38,38.72,31.67,26.24,25.81 . E-S 3 u:{ }^{1} \mathrm{H}$ NMR $(400 \mathrm{MHz}$, $\left.\mathrm{CDCl}_{3}\right) \delta 7.54(\mathrm{~s}, 1 \mathrm{H}), 3.77(\mathrm{~s}, 3 \mathrm{H}), 2.68(\mathrm{tt}, J=12.0,3.5 \mathrm{~Hz}, 1 \mathrm{H}), 1.90-1.52(\mathrm{~m}, 7 \mathrm{H})$, $1.35-1.14(\mathrm{~m}, 3 \mathrm{H}) .{ }^{13} \mathrm{C} \mathrm{NMR}\left(101 \mathrm{MHz}, \mathrm{CDCl}_{3}\right) \delta 165.54,143.91,128.05,118.57$ (q, $J=322.2 \mathrm{~Hz})$, 52.29, 36.80, 30.04, 26.63, 25.77. HRMS (ESI) Calcd for $\left[\mathrm{C}_{11} \mathrm{H}_{15} \mathrm{~F}_{3} \mathrm{NaO}_{5} \mathrm{~S}, \mathrm{M}+\mathrm{Na}\right]^{+}:$339.0484; Found: 339.0488 . 


\section{methyl (E)-2-phenyl-3-(((trifluoromethyl)sulfonyl)oxy)acrylate (S3v)}

TfO $91 \%$ yield for two steps, only $(E)-\mathbf{S 3 v}$ obtained, the configuration of

$\mathrm{Ph} \mathrm{CO}_{2} \mathrm{Me} \mathbf{S 3 v}$ was inferred from S3a. Yellow oil. ${ }^{1} \mathrm{H} \mathrm{NMR}\left(400 \mathrm{MHz}, \mathrm{CDCl}_{3}\right) \delta$ $7.86(\mathrm{~s}, 1 \mathrm{H}), 7.45-7.35(\mathrm{~m}, 3 \mathrm{H}), 7.30-7.25(\mathrm{~m}, 2 \mathrm{H}), 3.81$ (s, 3H). ${ }^{13} \mathrm{C}$ NMR $(101$ $\left.\mathrm{MHz}, \mathrm{CDCl}_{3}\right) \delta 165.18,144.33,129.53,129.18,129.14,128.43,124.24,118.46(\mathrm{q}, J=$ 322.2 Hz), 52.84. HRMS (ESI) Calcd for $\left[\mathrm{C}_{11} \mathrm{H}_{13} \mathrm{~F}_{3} \mathrm{NO}_{5} \mathrm{~S}, \mathrm{M}+\mathrm{NH}_{4}\right]^{+}:$328.0461; Found: 328.0460.

methyl (E)-2-(4-methoxyphenyl)-3-(((trifluoromethyl)sulfonyl)oxy)acrylate (S3w)<smiles>COc1ccc(/C(=C\O)C(=O)O[Na])cc1</smiles>
$89 \%$ yield for two steps, only (E)-S3w obtained, the configuration of S3w was inferred from S3a. Yellow oil. ${ }^{1} \mathrm{H}$ NMR (400 MHz, $\left.\mathrm{CDCl}_{3}\right) \delta 7.82(\mathrm{~s}, 1 \mathrm{H}), 7.25-7.20(\mathrm{~m}, 2 \mathrm{H})$, $6.95-6.91(\mathrm{~m}, 2 \mathrm{H}), 3.81(\mathrm{~s}, 6 \mathrm{H}) .{ }^{13} \mathrm{C} \mathrm{NMR}\left(101 \mathrm{MHz}, \mathrm{CDCl}_{3}\right) \delta 165.41,160.15,143.69$, 130.95, 123.80, 121.17, $118.33(\mathrm{q}, J=322.2 \mathrm{~Hz}), 113.84,55.24,52.73$. HRMS (ESI) Calcd for $\left[\mathrm{C}_{12} \mathrm{H}_{11} \mathrm{~F}_{3} \mathrm{NaO}_{6} \mathrm{~S}, \mathrm{M}+\mathrm{Na}\right]^{+}$: 363.0121; Found: 363.0123 .

methyl (E)-2-(4-chlorophenyl)-3-(((trifluoromethyl)sulfonyl)oxy)acrylate (S3x) $90 \%$ yield for two steps, only $(E)-\mathbf{S 3 x}$ obtained, the
configuration of $\mathbf{S 3 x}$ was inferred from S3a. Yellow oil. ${ }^{1} \mathrm{H}$
$\mathrm{NMR}\left(400 \mathrm{MHz}, \mathrm{CDCl}_{3}\right) \delta 7.87(\mathrm{~s}, 1 \mathrm{H}), 7.41-7.36(\mathrm{~m}, 2 \mathrm{H})$, $7.30-7.21(\mathrm{~m}, 2 \mathrm{H}), 3.83$ (s, 3H). ${ }^{13} \mathrm{C} \mathrm{NMR}\left(101 \mathrm{MHz}, \mathrm{CDCl}_{3}\right) \delta 164.86,144.73,135.41$, 131.04, 128.83, 127.59, 123.08, 118.43 (q, $J=322.2 \mathrm{~Hz}$ ), 53.01. HRMS (ESI) Calcd for $\left[\mathrm{C}_{11} \mathrm{H}_{8} \mathrm{ClF}_{3} \mathrm{NaO}_{5} \mathrm{~S}, \mathrm{M}+\mathrm{Na}\right]^{+}: 366.9625$; Found: 366.9629 .

methyl 2-(4-(trifluoromethyl)phenyl)-3-(((trifluoromethyl)sulfonyl)oxy)acrylate (S3y)<smiles>COC(=O)/C(=C/O)c1ccc(C(F)(F)F)cc1</smiles>
$85 \%$ yield for two steps. $Z / E=85: 15$, the configuration of $\mathbf{S 3 y}$ was inferred from S3a. Yellow oil. Z-S3y: ${ }^{1} \mathrm{H}$ NMR (400 MHz, $\left.\mathrm{CDCl}_{3}\right) \delta 7.66(\mathrm{~d}, J=8.2 \mathrm{~Hz}, 2 \mathrm{H}), 7.49(\mathrm{~d}, J=8.1 \mathrm{~Hz}, 2 \mathrm{H})$, $7.12(\mathrm{~s}, 1 \mathrm{H}), 3.90(\mathrm{~s}, 3 \mathrm{H}) \cdot{ }^{13} \mathrm{C}$ NMR $\left(101 \mathrm{MHz}, \mathrm{CDCl}_{3}\right) \delta$ 
164.59, 139.36, 134.81, 131.85 (q, $J=33.1 \mathrm{~Hz}), 128.75,125.98$ (q, $J=4.0 \mathrm{~Hz}), 125.03$, 122.53, 118.65 (q, $J=322.2 \mathrm{~Hz})$, 53.04. E-S3y: ${ }^{1} \mathrm{H}$ NMR $\left(400 \mathrm{MHz}, \mathrm{CDCl}_{3}\right) \delta 7.93$ (s, 1H), $7.69(\mathrm{~d}, J=8.1 \mathrm{~Hz}, 2 \mathrm{H}), 7.41(\mathrm{~d}, J=8.1 \mathrm{~Hz}, 2 \mathrm{H}), 3.85(\mathrm{~s}, 3 \mathrm{H}) .{ }^{13} \mathrm{C}$ NMR $(101$ $\left.\mathrm{MHz}, \mathrm{CDCl}_{3}\right) \delta 163.67,145.24,132.89,131.85(\mathrm{q}, J=33.1 \mathrm{~Hz}), 130.14,125.48(\mathrm{q}, J$ $=4.0 \mathrm{~Hz}), 125.24,122.87,118.22(\mathrm{q}, J=322.2 \mathrm{~Hz}), 53.08$. HRMS (ESI) Calcd for $\left[\mathrm{C}_{12} \mathrm{H}_{12} \mathrm{~F}_{6} \mathrm{NO}_{5} \mathrm{~S}, \mathrm{M}+\mathrm{NH}_{4}\right]^{+}:$396.0335; Found: 396.0309 .

(E)-2-benzyl-3-(dimethylaminoborane)prop-1-en-1-yl trifluoromethanesulfonate (4a)

$\begin{array}{ll}\text { TfO } & \begin{array}{l}\mathrm{BH}_{3} \\ \mathrm{NMe}_{2}\end{array} \quad \mathbf{4 a} \text { was confirmed by the NOESY NMR. White solid, mp: } 81-83^{\circ} \mathrm{C} \text {. }\end{array}$ ${ }^{1} \mathrm{H}$ NMR (400 MHz, $\left.\mathrm{CDCl}_{3}\right) \delta 7.37-7.22(\mathrm{~m}, 3 \mathrm{H}), 7.17$ (d, J=7.1 Hz, 2H), 6.82 (s, 1H), 3.87 (s, 2H), 3.23 (s, 2H), $2.60(\mathrm{~s}, 6 \mathrm{H}) .{ }^{13} \mathrm{C} \mathrm{NMR}\left(101 \mathrm{MHz}, \mathrm{CDCl}_{3}\right) \delta 138.83$, 136.50, 129.06, 128.99, 127.22, 124.34, 118.61 (q, $J=322.2 \mathrm{~Hz}), 62.31,52.06,34.72$. HRMS (ESI) Calcd for $\left[\mathrm{C}_{13} \mathrm{H}_{19} \mathrm{BF}_{3} \mathrm{NNaO}_{3} \mathrm{~S}, \mathrm{M}+\mathrm{Na}\right]^{+}$: 360.1023; Found: 360.1026.

\section{(E)-2-benzyl-3-(butyl(methyl)aminoborane)prop-1-en-1-yl}

\section{trifluoromethanesulfonate (4b)}

TfO $\quad \mathrm{BH}_{3} \quad 62 \%$ yield for four steps. White solid, mp: $88-90{ }^{\circ} \mathrm{C} .{ }^{1} \mathrm{H}$ NMR

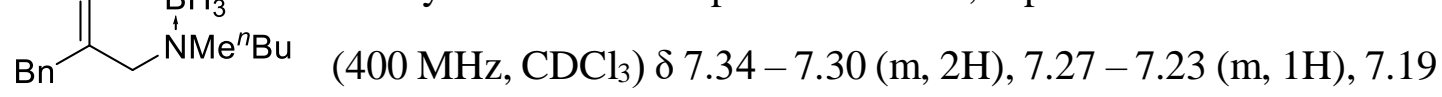
$-7.17(\mathrm{~m}, 2 \mathrm{H}), 6.80(\mathrm{~s}, 1 \mathrm{H}), 3.89$ (s, 2H), $3.20(\mathrm{dd}, J=33.2,32.8 \mathrm{~Hz}, 2 \mathrm{H}), 2.77-2.72$ (m, 2H), $2.53(\mathrm{~s}, 3 \mathrm{H}), 1.68-1.57(\mathrm{~m}, 2 \mathrm{H}), 1.33-1.24(\mathrm{~m}, 2 \mathrm{H}), 0.94(\mathrm{t}, J=7.2 \mathrm{~Hz}, 3 \mathrm{H})$. ${ }^{13} \mathrm{C} \mathrm{NMR}\left(101 \mathrm{MHz}, \mathrm{CDCl}_{3}\right) \delta 138.69,136.78,129.03,129.00,127.16,124.46,118.63$ (q, $J=322.2 \mathrm{~Hz}), 62.99,59.55,49.36,34.80,25.58,20.45,13.85$. HRMS (ESI) Calcd for $\left[\mathrm{C}_{16} \mathrm{H}_{25} \mathrm{BF}_{3} \mathrm{NNaO}_{3} \mathrm{~S}, \mathrm{M}+\mathrm{Na}\right]^{+}:$402.1493; Found: 402.1497.

\section{(E)-2-benzyl-3-(benzyl(methyl)aminoborane)prop-1-en-1-yl}

\section{trifluoromethanesulfonate $(4 \mathrm{c})$}

$56 \%$ yield for four steps. White solid, mp: $107-109{ }^{\circ} \mathrm{C} .{ }^{1} \mathrm{H}$ NMR
$\begin{aligned} & \mathrm{Bn}_{4}^{4} \mathrm{H}_{3} \\ & \mathrm{NMeBn}\end{aligned}$ 
$-7.13(\mathrm{~m}, 2 \mathrm{H}), 6.67(\mathrm{~s}, 1 \mathrm{H}), 4.09-4.03(\mathrm{~m}, 2 \mathrm{H}), 3.97(\mathrm{~d}, J=13.2 \mathrm{~Hz}, 1 \mathrm{H}), 3.85(\mathrm{~d}, J$ $=14.8 \mathrm{~Hz}, 1 \mathrm{H}), 3.27(\mathrm{~d}, J=12.8 \mathrm{~Hz}, 1 \mathrm{H}), 2.96(\mathrm{~d}, J=12.8 \mathrm{~Hz}, 1 \mathrm{H}), 2.49(\mathrm{~s}, 3 \mathrm{H}) .{ }^{13} \mathrm{C}$ NMR (101 MHz, $\left.\mathrm{CDCl}_{3}\right) \delta 138.40,137.13,132.65,130.52,129.49,129.17,128.98$, 128.67, 127.05, 124.66, 118.62 (q, $J=322.2 \mathrm{~Hz}), 68.86,59.12,47.05,35.03$. HRMS (ESI) Calcd for $\left[\mathrm{C}_{19} \mathrm{H}_{23} \mathrm{BF}_{3} \mathrm{NNaO}_{3} \mathrm{~S}, \mathrm{M}+\mathrm{Na}\right]^{+}$: 436.1341 ; Found: 436.1336.

\section{2-benzyl-3-(azetidinborane-1-yl)prop-1-en-1-yl trifluoromethanesulfonate (4d)}<smiles>[B]C1(C/C(=C\[OH2+])Cc2ccccc2)CCC1</smiles>

Z-4d: $29 \%$ yield for four steps. White solid, mp: $81-83{ }^{\circ} \mathrm{C} .{ }^{1} \mathrm{H}$ NMR $\left(400 \mathrm{MHz}, \mathrm{CDCl}_{3}\right) \delta 7.38-7.28(\mathrm{~m}, 2 \mathrm{H}), 7.31-7.21(\mathrm{~m}, 1 \mathrm{H}), 7.20-$ $7.11(\mathrm{~m}, 2 \mathrm{H}), 6.85(\mathrm{~s}, 1 \mathrm{H}), 3.77(\mathrm{~s}, 2 \mathrm{H}), 3.74-3.64(\mathrm{~m}, 2 \mathrm{H}), 3.64-$ $3.54(\mathrm{~m}, 2 \mathrm{H}), 3.24(\mathrm{~s}, 2 \mathrm{H}), 2.85-2.63(\mathrm{~m}, 1 \mathrm{H}), 2.09-1.95(\mathrm{~m}, 1 \mathrm{H}) .{ }^{13} \mathrm{C}$ NMR $(101$ $\left.\mathrm{MHz}, \mathrm{CDCl}_{3}\right) \delta 137.19,136.50,129.10,128.92,127.17,124.75,118.64(\mathrm{q}, J=322.2$ $\mathrm{Hz}), 62.74,62.12,33.38,14.88$.

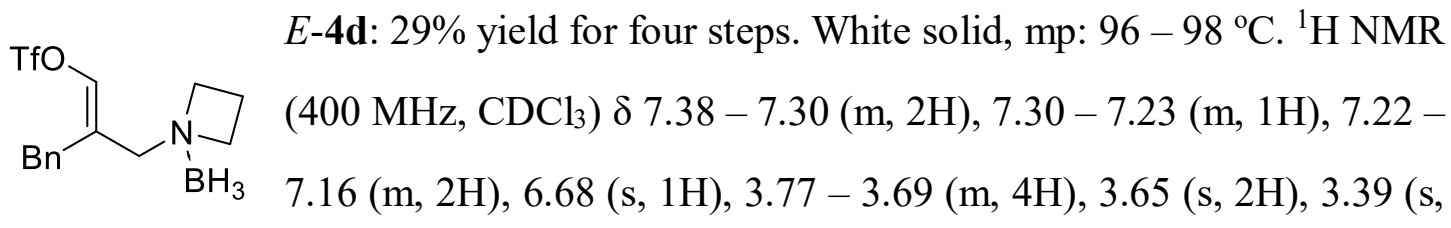
$2 \mathrm{H}), 2.82-2.62(\mathrm{~m}, 1 \mathrm{H}), 2.10-2.00(\mathrm{~m}, 1 \mathrm{H}) .{ }^{13} \mathrm{C} \mathrm{NMR}\left(101 \mathrm{MHz}, \mathrm{CDCl}_{3}\right) \delta 136.61$, $136.47,129.18,129.05,127.34,124.91,118.61$ (q, $J=322.2 \mathrm{~Hz}), 62.81,58.75,37.75$, 14.96. HRMS (ESI) Calcd for $\left[\mathrm{C}_{14} \mathrm{H}_{18} \mathrm{BF}_{3} \mathrm{NO}_{3} \mathrm{~S}, \mathrm{M}-\mathrm{H}\right]^{+}:$348.1047; Found: 348.1040.

\section{2-benzyl-3-(pyrrolidinborane-1-yl)prop-1-en-1-yl trifluoromethanesulfonate (4e)}<smiles>[B][N+]1(C/C(=C\[OH2+])Cc2ccccc2)CCCC1</smiles>

Z-4e: $34 \%$ yield for four steps. White solid, mp: $60-62{ }^{\circ} \mathrm{C} .{ }^{1} \mathrm{H}$ NMR $\left(400 \mathrm{MHz}, \mathrm{CDCl}_{3}\right) \delta 7.36-7.29(\mathrm{~m}, 2 \mathrm{H}), 7.28-7.24(\mathrm{~m}, 1 \mathrm{H}), 7.22-$ $7.16(\mathrm{~m}, 2 \mathrm{H}), 6.85(\mathrm{~s}, 1 \mathrm{H}), 3.94(\mathrm{~s}, 2 \mathrm{H}), 3.23(\mathrm{~s}, 2 \mathrm{H}), 3.33-3.05(\mathrm{~m}$, 2H), $2.82-2.63(\mathrm{~m}, 2 \mathrm{H}), 2.45-2.14(\mathrm{~m}, 2 \mathrm{H}), 1.94-1.80(\mathrm{~m}, 2 \mathrm{H}) .{ }^{13} \mathrm{C}$ NMR $(101$ $\left.\mathrm{MHz}, \mathrm{CDCl}_{3}\right) \delta 138.14,136.91,129.05,128.98,127.06,125.42,118.62$ (q, $J=322.2$ Hz), 61.81, 61.11, 34.59, 22.51.

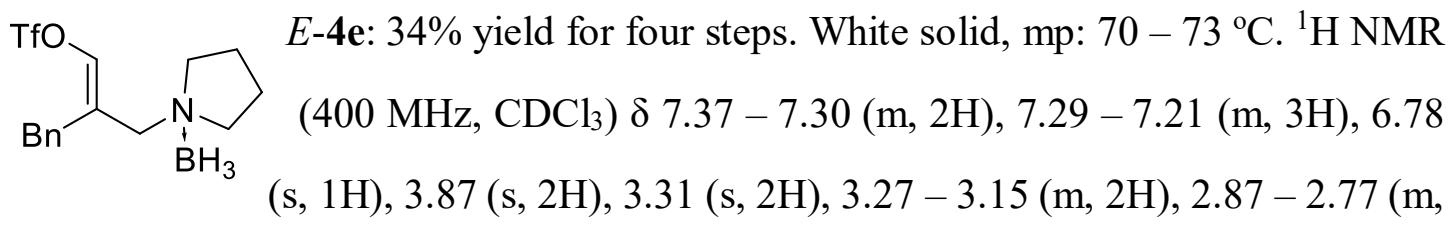


2H), $2.25-2.16(\mathrm{~m}, 2 \mathrm{H}), 1.99-1.90(\mathrm{~m}, 2 \mathrm{H}) .{ }^{13} \mathrm{C} \mathrm{NMR}\left(101 \mathrm{MHz}, \mathrm{CDCl}_{3}\right) \delta 137.15$, 136.97, 129.38, 128.95, 127.24, 125.68, 118.58 (q, $J=322.2 \mathrm{~Hz}), 62.32,56.62,39.06$, 22.55. HRMS (ESI) Calcd for $\left[\mathrm{C}_{15} \mathrm{H}_{20} \mathrm{BF}_{3} \mathrm{NO}_{3} \mathrm{~S}, \mathrm{M}-\mathrm{H}\right]^{+}$: 362.1204 ; Found: 362.1208 .

(Z)-2-benzyl-3-(piperidinborane-1-yl)prop-1-en-1-yl trifluoromethanesulfonate (4f)<smiles>[B][N+]1(C/C(=C\[OH+])Cc2ccccc2)CCCCC1</smiles>
$79 \%$ yield for four steps. White solid, mp: $85-88{ }^{\circ} \mathrm{C} .{ }^{1} \mathrm{H}$ NMR (400 $\left.\mathrm{MHz}, \mathrm{CDCl}_{3}\right) \delta 7.43-7.28(\mathrm{~m}, 2 \mathrm{H}), 7.28-7.24(\mathrm{~m}, 1 \mathrm{H}), 7.23-7.13$ $(\mathrm{m}, 2 \mathrm{H}), 6.80(\mathrm{~s}, 1 \mathrm{H}), 3.96(\mathrm{~s}, 2 \mathrm{H}), 3.21(\mathrm{~s}, 2 \mathrm{H}), 3.10-2.91(\mathrm{~m}, 2 \mathrm{H})$, $2.81-2.61(\mathrm{~m}, 2 \mathrm{H}), 2.07-1.89(\mathrm{~m}, 2 \mathrm{H}), 1.64-1.45(\mathrm{~m}, 4 \mathrm{H}) .{ }^{13} \mathrm{C}$ NMR $(101 \mathrm{MHz}$, $\left.\mathrm{CDCl}_{3}\right) \delta 138.37,137.18,129.14,128.97,127.06,124.47,118.66(\mathrm{q}, J=322.2 \mathrm{~Hz})$, 59.02, 35.38, 22.79, 20.74. HRMS (ESI) Calcd for $\left[\mathrm{C}_{16} \mathrm{H}_{23} \mathrm{BF}_{3} \mathrm{KNO}_{3} \mathrm{~S}, \mathrm{M}+\mathrm{K}\right]^{+}$: 416.1075; Found: 416.1070.

\section{2-benzyl-3-(azepanborane-1-yl)prop-1-en-1-yl trifluoromethanesulfonate (4g)}<smiles>[B][N+]1(C/C(Br)=C\[OH2+])CCCCCC1</smiles>

Z-4g: $30 \%$ yield for four steps. White solid, mp: $95-97{ }^{\circ} \mathrm{C} .{ }^{1} \mathrm{H}$ NMR $\left(400 \mathrm{MHz} \mathrm{CDCl}_{3}\right) \delta 7.31(\mathrm{dd}, J=8.0,6.4 \mathrm{~Hz}, 2 \mathrm{H}), 7.28-7.24(\mathrm{~m}$, 1H), $7.22-7.16(\mathrm{~m}, 2 \mathrm{H}), 6.79(\mathrm{~s}, 1 \mathrm{H}), 3.95(\mathrm{~s}, 2 \mathrm{H}), 3.16(\mathrm{~s}, 2 \mathrm{H})$, $3.11(\mathrm{dd}, J=13.9,8.6 \mathrm{~Hz}, 2 \mathrm{H}), 2.83(\mathrm{dd}, J=13.8,8.8 \mathrm{~Hz}, 2 \mathrm{H}), 1.93-1.80(\mathrm{~m}, 2 \mathrm{H})$, $1.68-1.50(\mathrm{~m}, 6 \mathrm{H}) .{ }^{13} \mathrm{C} \mathrm{NMR}\left(101 \mathrm{MHz}, \mathrm{CDCl}_{3}\right) \delta 138.81,137.16,129.16,128.98$, 127.07, 124.82, $118.66(\mathrm{q}, J=322.2 \mathrm{~Hz}), 61.21,60.43,35.05,29.24,22.41$.<smiles>[B][N+]1(C/C(Br)=C\O)CCCCCC1</smiles>

E-4g: $27 \%$ yield for four steps. White solid, mp: $100-102{ }^{\circ} \mathrm{C} .{ }^{1} \mathrm{H}$ NMR (400 MHz, $\left.\mathrm{CDCl}_{3}\right) \delta 7.32(\mathrm{dd}, J=8.0,6.4 \mathrm{~Hz}, 2 \mathrm{H}), 7.30-$ $7.20(\mathrm{~m}, 3 \mathrm{H}), 6.79(\mathrm{~s}, 1 \mathrm{H}), 3.90(\mathrm{~s}, 2 \mathrm{H}), 3.21(\mathrm{~s}, 2 \mathrm{H}), 3.09(\mathrm{dd}, J=$ 13.8, 8.1 Hz, 2H), $2.84(\mathrm{dd}, J=13.8,8.9 \mathrm{~Hz}, 2 \mathrm{H}), 1.94-1.80(\mathrm{~m}, 2 \mathrm{H}), 1.69-1.50$ (m, $6 \mathrm{H}) .{ }^{13} \mathrm{C} \mathrm{NMR}\left(101 \mathrm{MHz}, \mathrm{CDCl}_{3}\right) \delta 137.71,137.20,129.42,128.90,127.18,125.55$, $118.62(\mathrm{q}, J=322.2 \mathrm{~Hz}), 61.59,56.31,39.26,29.53,22.42$. HRMS (ESI) Calcd for $\left[\mathrm{C}_{17} \mathrm{H}_{25} \mathrm{BF}_{3} \mathrm{NNaO}_{3} \mathrm{~S}, \mathrm{M}+\mathrm{Na}\right]^{+}:$414.1493; Found: 414.1486. 
(Z)-2-benzyl-3-(morpholinoborane-1-yl)prop-1-en-1-yl trifluoromethanesulfonate

(4h)<smiles>[B][N+]1(C/C(Br)=C\[OH2+])CCOCC1</smiles>

$74 \%$ for four steps. White solid, mp: $52-55{ }^{\circ} \mathrm{C} .{ }^{1} \mathrm{H} \mathrm{NMR}(400 \mathrm{MHz}$, $\left.\mathrm{CDCl}_{3}\right) \delta 7.32(\mathrm{t}, J=7.4 \mathrm{~Hz}, 2 \mathrm{H}), 7.26(\mathrm{t}, J=7.3 \mathrm{~Hz}, 1 \mathrm{H}), 7.19(\mathrm{~d}, J$ $=7.5 \mathrm{~Hz}, 2 \mathrm{H}), 6.85(\mathrm{~s}, 1 \mathrm{H}), 4.26(\mathrm{t}, J=11.4 \mathrm{~Hz}, 2 \mathrm{H}), 3.95(\mathrm{~s}, 2 \mathrm{H})$, $3.69(\mathrm{dt}, J=12.8,3.4 \mathrm{~Hz}, 2 \mathrm{H}), 3.25(\mathrm{~s}, 2 \mathrm{H}), 2.96(\mathrm{~d}, J=11.9 \mathrm{~Hz}, 2 \mathrm{H}), 2.70$ (dt, $J=$ 12.8, $2.8 \mathrm{~Hz}, 2 \mathrm{H}) .{ }^{13} \mathrm{C} \mathrm{NMR}\left(101 \mathrm{MHz}, \mathrm{CDCl}_{3}\right) \delta$ 138.93, 136.81, 129.11, 129.07, 127.21, 123.44, 118.65 (q, $J=322.2 \mathrm{~Hz}), 64.60,61.92,58.35,35.32$. HRMS (ESI) Calcd for $\left[\mathrm{C}_{15} \mathrm{H}_{20} \mathrm{BF}_{3} \mathrm{NO}_{4} \mathrm{~S}, \mathrm{M}-\mathrm{H}\right]^{+}:$378.1153; Found: 378.1158.

(Z)-2-benzyl-3-(benzylaminoborane)prop-1-en-1-yl trifluoromethanesulfonate (4i) $\begin{array}{ll}\begin{array}{l}\mathrm{BH}_{4}^{4} \\ \mathrm{NHBn}\end{array} & 53 \% \text { yield for four steps. White solid, mp: } 71-73{ }^{\circ} \mathrm{C} .{ }^{1} \mathrm{H} \text { NMR }(400 \\ \left.\mathrm{MHz}, \mathrm{CDCl}_{3}\right) \delta 7.34-7.23(\mathrm{~m}, 6 \mathrm{H}), 7.13-7.04(\mathrm{~m}, 4 \mathrm{H}), 6.60(\mathrm{~s}, 1 \mathrm{H}),\end{array}$ $4.21(\mathrm{dd}, J=13.8,3.5 \mathrm{~Hz}, 1 \mathrm{H}), 3.89(\mathrm{~s}, 1 \mathrm{H}), 3.53(\mathrm{dd}, J=13.7,9.2 \mathrm{~Hz}, 1 \mathrm{H}), 3.49(\mathrm{~d}, J$ $=5.5 \mathrm{~Hz}, 2 \mathrm{H}), 3.30(\mathrm{dd}, J=13.0,2.8 \mathrm{~Hz}, 1 \mathrm{H}), 3.13(\mathrm{dd}, J=12.9,9.7 \mathrm{~Hz}, 1 \mathrm{H}) .{ }^{13} \mathrm{C}$ NMR (101 MHz, $\left.\mathrm{CDCl}_{3}\right) \delta 135.59,135.56,133.30,129.21,129.15,129.00,128.84$, 127.52, 125.49, 118.43 (q, $J=322.2 \mathrm{~Hz}$ ), 60.59, 49.20, 37.26. HRMS (ESI) Calcd for $\left[\mathrm{C}_{18} \mathrm{H}_{21} \mathrm{BF}_{3} \mathrm{KNO}_{3} \mathrm{~S}, \mathrm{M}+\mathrm{K}\right]^{+}$: 438.0919; Found: 438.0913.

(Z)-2-benzyl-3-((S)-benzylaminoborane)

prop-1-en-1-yl trifluoromethanesulfonate $(\mathbf{4 j})$<smiles>C[C](c1ccccc1)N(C)[C@@H](C)N(C)Cc1ccccc1</smiles>
$67 \%$ for four steps. White solid, mp: $82-85^{\circ} \mathrm{C} .{ }^{1} \mathrm{H}$ NMR $(400 \mathrm{MHz}$, $\left.\mathrm{CDCl}_{3}\right) \delta 7.35-7.25(\mathrm{~m}, 6 \mathrm{H}), 7.09-7.02(\mathrm{~m}, 2 \mathrm{H}), 6.95-6.87(\mathrm{~m}$, 2H), $6.67(\mathrm{~s}, 1 \mathrm{H}), 3.73(\mathrm{p}, J=7.0 \mathrm{~Hz}, 1 \mathrm{H}), 3.65(\mathrm{~d}, J=15.7 \mathrm{~Hz}$, 1H), 3.47 (d, $J=15.7 \mathrm{~Hz}, 1 \mathrm{H}), 3.33(\mathrm{~s}, 1 \mathrm{H}), 3.15$ (dd, $J=13.4,3.5 \mathrm{~Hz}, 1 \mathrm{H}), 3.03$ (dd, $J=13.3,8.6 \mathrm{~Hz}, 1 \mathrm{H}), 1.50(\mathrm{~d}, J=6.8 \mathrm{~Hz}, 3 \mathrm{H}) .{ }^{13} \mathrm{C} \mathrm{NMR}\left(101 \mathrm{MHz}, \mathrm{CDCl}_{3}\right) \delta 138.94$, $136.24,135.66,129.50,129.18,128.91,128.86,127.75,127.49,124.30,118.61$ (q, $J=$ 322.2 Hz), 64.42, 53.99, 32.63, 19.24. HRMS (ESI) Calcd for $\left[\mathrm{C}_{19} \mathrm{H}_{23} \mathrm{BF}_{3} \mathrm{KNO}_{3} \mathrm{~S}, \mathrm{M}+\right.$ $\mathrm{K}]^{+}$: 452.1075; Found: 452.1066 . 
(Z)-2-benzyl- 3-((3S,4R)-3-((benzo[d][1,3]dioxol-5-yloxy)methyl)-4-(4-

fluorophenyl)piperidin-1-yl aminoborane)prop-1-en-1-yl

trifluoromethanesulfonate $(4 k)$

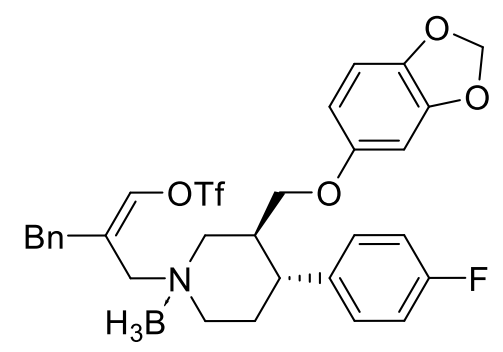

$61 \%$ yield for four steps. White solid, mp: $51-53{ }^{\circ} \mathrm{C}$. ${ }^{1} \mathrm{H} \mathrm{NMR}\left(400 \mathrm{MHz}, \mathrm{CDCl}_{3}\right) \delta 7.35-7.28(\mathrm{~m}, 2 \mathrm{H}), 7.28$ $-7.20(\mathrm{~m}, 3 \mathrm{H}), 7.17$ (ddd, $J=9.5,5.8,3.3 \mathrm{~Hz}, 2 \mathrm{H}), 6.98$ (td, $J=8.7,2.1 \mathrm{~Hz}, 2 \mathrm{H}), 6.72(\mathrm{~s}, 1 \mathrm{H}), 6.62(\mathrm{dd}, J=8.5$, $1.3 \mathrm{~Hz}, 1 \mathrm{H}), 6.58(\mathrm{~s}, 1 \mathrm{H}), 6.33$ (dd, $J=4.2,2.5 \mathrm{~Hz}, 1 \mathrm{H})$, 6.12 (ddd, $J=8.5,4.1,2.5 \mathrm{~Hz}, 1 \mathrm{H}), 5.86(\mathrm{~s}, 2 \mathrm{H}), 3.60(\mathrm{~s}, 1 \mathrm{H}), 3.58-3.52(\mathrm{~m}, 1 \mathrm{H})$, $3.45(\mathrm{~s}, 1 \mathrm{H}), 3.44-3.37(\mathrm{~m}, 1 \mathrm{H}), 3.14-3.05(\mathrm{~m}, 1 \mathrm{H}), 3.00(\mathrm{~s}, 1 \mathrm{H}), 2.88-2.80(\mathrm{~m}, 2 \mathrm{H})$, $2.51-2.35(\mathrm{~m}, 1 \mathrm{H}), 2.25-2.11(\mathrm{~m}, 1 \mathrm{H}), 2.11-1.92(\mathrm{~m}, 2 \mathrm{H}), 1.86-1.71(\mathrm{~m}, 2 \mathrm{H}) .{ }^{13} \mathrm{C}$ NMR (101 MHz, $\left.\mathrm{CDCl}_{3}\right) \delta 163.12,162.88,160.69,160.45,154.48,154.18,148.31$, 148.27, 142.02, 141.71, 139.79 (d, $J=3.2 \mathrm{~Hz}), 138.35$ (d, $J=3.2 \mathrm{~Hz}), 138.03,137.63$, $136.91,133.48,132.90,131.19,129.37,129.22$, 129.15, 129.09, 129.03, 128.97, $128.89,128.71,127.35,126.92,126.76,123.97,118.68$ (q, $J=322.2 \mathrm{~Hz}), 118.57$ (q, $J$ = 322.2 Hz), 115.87, 115.66, 115.64, 115.43, 107.99, 107.96, 105.83, 105.74, 101.28, 101.21, 98.17, 98.10, 69.74, 68.59, 62.60, 62.11, 59.68, 57.55, 53.88, 53.76, 44.17, 42.33, 39.75, 37.85, 36.25, 34.62, 29.84, 29.24. HRMS (ESI) Calcd for $\left[\mathrm{C}_{30} \mathrm{H}_{32} \mathrm{BF}_{4} \mathrm{NNaO}_{6} \mathrm{~S}, \mathrm{M}+\mathrm{Na}\right]^{+}:$644.1872; Found: 644.1869 .

\section{(Z)-2-benzyl-3-((3-(10,11-dihydro-5H-dibenzo[ $a, d][7]$ annulen-5-}

ylidene)propyl)(methyl)aminoborane)prop-1-en-1-yl trifluoromethanesulfonate (4I)<smiles></smiles>
$55 \%$ for four steps. White solid, mp: $104-107^{\circ} \mathrm{C}$. ${ }^{1} \mathrm{H}$ NMR $\left(400 \mathrm{MHz}, \mathrm{CDCl}_{3}\right) \delta 7.32-7.26(\mathrm{~m}, 2 \mathrm{H}), 7.26$ $-7.21(\mathrm{~m}, 2 \mathrm{H}), 7.21-7.17(\mathrm{~m}, 2 \mathrm{H}), 7.16-7.12(\mathrm{~m}, 3 \mathrm{H})$, $7.10-7.07(\mathrm{~m}, 2 \mathrm{H}), 7.03(\mathrm{t}, J=7.1 \mathrm{~Hz}, 2 \mathrm{H}), 6.72(\mathrm{~s}$,

1H), $5.70(\mathrm{t}, J=7.3 \mathrm{~Hz}, 1 \mathrm{H}), 3.90-3.68(\mathrm{~m}, 2 \mathrm{H}), 3.40-3.19(\mathrm{~m}, 2 \mathrm{H}), 3.19-3.06(\mathrm{~m}$, $1 \mathrm{H}), 3.06-2.80(\mathrm{~m}, 3 \mathrm{H}), 2.80-2.61(\mathrm{~m}, 2 \mathrm{H}), 2.59-2.44(\mathrm{~m}, 2 \mathrm{H}), 2.39(\mathrm{~d}, J=14.5$ $\mathrm{Hz}, 3 \mathrm{H}) .{ }^{13} \mathrm{C} \mathrm{NMR}\left(101 \mathrm{MHz}, \mathrm{CDCl}_{3}\right) \delta 146.04,140.51,139.34,139.22,138.65$, 
$137.11,136.67,136.66,130.20,128.98,128.95,128.51,128.39,128.00,127.72$, $127.55,127.12,126.22,126.08,125.68,118.60$ (q, $J=322.2 \mathrm{~Hz}), 62.58,62.27,60.32$, 59.35, 49.30, 48.51, 34.77, 33.73, 32.02, 24.14, 24.00. HRMS (ESI) Calcd for $\left[\mathrm{C}_{30} \mathrm{H}_{33} \mathrm{BF}_{3} \mathrm{NNaO}_{3} \mathrm{~S}, \mathrm{M}+\mathrm{Na}\right]^{+}$: 578.2119; Found: 578.2102.

\section{(Z)-2-benzyl-3-(methyl(3-phenyl-3-(4-}

(trifluoromethyl)phenoxy)propyl)aminoborane)prop-1-en-1-yl

trifluoromethanesulfonate $(4 \mathrm{~m})$

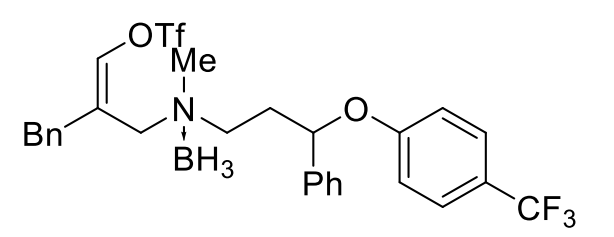

$58 \%$ yield for four steps. Colorless oil. ${ }^{1} \mathrm{H}$ NMR $\left(400 \mathrm{MHz}, \mathrm{CDCl}_{3}\right) \delta 7.43(\mathrm{~d}, J=8.4 \mathrm{~Hz}, 2 \mathrm{H}), 7.39$ $-7.21(\mathrm{~m}, 8 \mathrm{H}), 7.15(\mathrm{~d}, J=7.3 \mathrm{~Hz}, 2 \mathrm{H}), 6.87$ (d, $J$ $=8.4 \mathrm{~Hz}, 2 \mathrm{H}), 6.82(\mathrm{~d}, J=6.9 \mathrm{~Hz}, 1 \mathrm{H}), 5.20(\mathrm{t}, J$

$=6.3 \mathrm{~Hz}, 1 \mathrm{H}), 3.87(\mathrm{~s}, 2 \mathrm{H}), 3.32-3.14(\mathrm{~m}, 2 \mathrm{H}), 3.13-2.80(\mathrm{~m}, 2 \mathrm{H}), 2.54(\mathrm{~d}, J=13.5$ $\mathrm{Hz}, 3 \mathrm{H}), 2.42-2.29$ (m, 2H). ${ }^{13} \mathrm{C} \mathrm{NMR}\left(101 \mathrm{MHz}, \mathrm{CDCl}_{3}\right) \delta 160.08,139.79,139.00$, $138.94,136.56,136.51,129.20,129.12,128.99$, 128.95, 128.51, 127.29, 127.27, $127.00(\mathrm{q}, J=3.4 \mathrm{~Hz}), 125.71,124.13,123.48(\mathrm{q}, J=33.3 \mathrm{~Hz}), 118.65(\mathrm{q}, J=322.2$ Hz), 115.83, 78.59, 78.56, 61.03, 59.90, 59.84, 59.64, 50.14, 49.36, 34.82, 34.71, 33.15, 33.04. HRMS (ESI) Calcd for $\left[\mathrm{C}_{28} \mathrm{H}_{30} \mathrm{BF}_{6} \mathrm{NNaO}_{4} \mathrm{~S}, \mathrm{M}+\mathrm{Na}\right]^{+}$: 624.1785; Found: 624.1774 .

\section{(E)-3-(benzyl(methyl)aminoborane)-2-(4-chlorobenzyl)prop-1-en-1-yl}

\section{trifluoromethanesulfonate $(4 n)$}<smiles>[B]N(C/C(=C/O[Ga]c1ccc(Cl)cc1)Cc1ccccc1)Cc1ccccc1</smiles>
$49 \%$ yield for four steps. White solid, mp: $94-98{ }^{\circ} \mathrm{C} .{ }^{1} \mathrm{H}$

NMR (400 MHz, $\left.\mathrm{CDCl}_{3}\right) \delta 7.47-7.32(\mathrm{~m}, 3 \mathrm{H}), 7.26(\mathrm{~d}, J$ $=8.3 \mathrm{~Hz}, 4 \mathrm{H}), 7.07(\mathrm{~d}, J=8.2 \mathrm{~Hz}, 2 \mathrm{H}), 6.65(\mathrm{~s}, 1 \mathrm{H}), 4.06(\mathrm{t}, J=13.2 \mathrm{~Hz}, 2 \mathrm{H}), 3.98(\mathrm{~d}$, $J=13.4 \mathrm{~Hz}, 1 \mathrm{H}), 3.84(\mathrm{~d}, J=14.9 \mathrm{~Hz}, 1 \mathrm{H}), 3.22(\mathrm{~d}, J=12.8 \mathrm{~Hz}, 1 \mathrm{H}), 2.92(\mathrm{~d}, J=12.8$ $\mathrm{Hz}, 1 \mathrm{H}), 2.49$ (s, 3H). ${ }^{13} \mathrm{C} \mathrm{NMR}\left(101 \mathrm{MHz}, \mathrm{CDCl}_{3}\right) \delta 138.48,135.67,132.96,132.64$, 130.56, 130.40, 129.59, 129.12, 128.75, 124.42, 118.61 (q, $J=322.2$ Hz), 69.06, 58.86, 47.05, 34.32. HRMS (ESI) Calcd for $\left[\mathrm{C}_{19} \mathrm{H}_{22} \mathrm{BClF}_{3} \mathrm{NNaO}_{3} \mathrm{~S}, \mathrm{M}+\mathrm{Na}\right]^{+}$: 470.0946; Found: 470.0952. 


\section{3-(benzyl(methyl)aminoborane)-2-(4-(trifluoromethyl)benzyl)prop-1-en-1-yl}

\section{trifluoromethanesulfonate (4o)}

$\mathrm{F}_{3} \mathrm{C}$

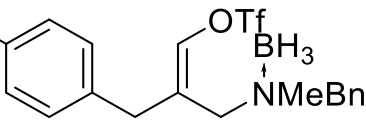

Z-4o: $20 \%$ yield for four steps, white solid, mp: $102-$

$104{ }^{\circ} \mathrm{C} .{ }^{1} \mathrm{H}$ NMR $\left(400 \mathrm{MHz}, \mathrm{CDCl}_{3}\right) \delta 7.57(\mathrm{~d}, J=8.0$ $\mathrm{Hz}, 2 \mathrm{H}), 7.42-7.29(\mathrm{~m}, 7 \mathrm{H}), 6.76(\mathrm{~s}, 1 \mathrm{H}), 4.16(\mathrm{~d}, J=14.8 \mathrm{~Hz}, 1 \mathrm{H}), 4.03$ (q, $J=13.2$ Hz, 2H), $3.74(\mathrm{~d}, J=15.2 \mathrm{~Hz}, 1 \mathrm{H}), 3.31(\mathrm{~d}, J=12.0 \mathrm{~Hz}, 1 \mathrm{H}), 3.02(\mathrm{~d}, J=12.0 \mathrm{~Hz}, 1 \mathrm{H})$, $2.55(\mathrm{~s}, 3 \mathrm{H}) .{ }^{13} \mathrm{C} \mathrm{NMR}\left(101 \mathrm{MHz}, \mathrm{CDCl}_{3}\right) \delta 141.21(\mathrm{~d}, J=1.2 \mathrm{~Hz}), 137.55,132.60$, $130.41,129.77,129.54,128.54,125.88(\mathrm{q}, J=3.9 \mathrm{~Hz}), 124.53,118.40(\mathrm{q}, J=321.8$ Hz), 69.62, 55.11, 47.90, 38.80.<smiles>[B]N(CC(=CO)Cc1ccc(C(F)(F)F)cc1)Cc1ccccc1</smiles>
E-4o: $20 \%$ yield for four steps, white solid, mp: $106-$ $108{ }^{\circ} \mathrm{C} .{ }^{1} \mathrm{H}$ NMR $\left(400 \mathrm{MHz}, \mathrm{CDCl}_{3}\right) \delta 7.55(\mathrm{~d}, J=8.0$ $\mathrm{Hz}, 2 \mathrm{H}), 7.42-7.35$ (m, 3H), $7.28-7.25$ (m, 4H), $6.68(\mathrm{~s}, 1 \mathrm{H}), 4.19-3.93(\mathrm{~m}, 4 \mathrm{H})$, $3.21(\mathrm{~d}, J=12.8 \mathrm{~Hz}, 1 \mathrm{H}), 2.92(\mathrm{~d}, J=12.4 \mathrm{~Hz}, 1 \mathrm{H}), 2.51(\mathrm{~s}, 3 \mathrm{H}) .{ }^{13} \mathrm{C} \mathrm{NMR}(101 \mathrm{MHz}$, $\left.\mathrm{CDCl}_{3}\right) \delta 141.36(\mathrm{~d}, J=1.5 \mathrm{~Hz}), 138.76,132.63,130.32,129.62,129.51,128.75$, $125.90(\mathrm{q}, J=3.7 \mathrm{~Hz}), 123.91,118.59$ (q, $J=322.2 \mathrm{~Hz}), 69.11,58.93,47.10,34.81$. HRMS (ESI) Calcd for $\left[\mathrm{C}_{20} \mathrm{H}_{22} \mathrm{BF}_{6} \mathrm{NNaO}_{3} \mathrm{~S}, \mathrm{M}+\mathrm{Na}\right]^{+}:$504.1210; Found: 504.1215.

\section{(E)-3-(benzyl(methyl)aminoborane)-2-(4-methoxybenzyl)prop-1-en-1-yl trifluoromethanesulfonate $(4 p)$}

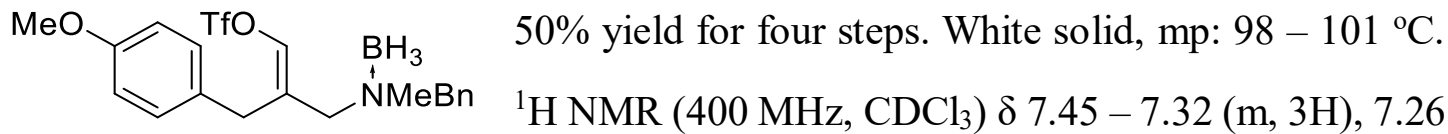
$(\mathrm{dd}, J=6.6,0.9 \mathrm{~Hz}, 2 \mathrm{H}), 7.10-7.04(\mathrm{~m}, 2 \mathrm{H}), 6.88-6.79(\mathrm{~m}, 2 \mathrm{H}), 6.64(\mathrm{~s}, 1 \mathrm{H}), 4.07$ (d, $J=13.4 \mathrm{~Hz}, 1 \mathrm{H}), 4.03-3.93(\mathrm{~m}, 2 \mathrm{H}), 3.80$ (s, 3H), 3.79 (d, $J=11.6 \mathrm{~Hz}, 1 \mathrm{H}), 3.26$ $(\mathrm{d}, J=12.8 \mathrm{~Hz}, 1 \mathrm{H}), 2.94(\mathrm{~d}, J=12.8 \mathrm{~Hz}, 1 \mathrm{H}), 2.48(\mathrm{~s}, 3 \mathrm{H}) .{ }^{13} \mathrm{C}$ NMR $(101 \mathrm{MHz}$, $\left.\mathrm{CDCl}_{3}\right) \delta 158.64,138.10,132.66,130.54,130.22,129.46,129.00,128.64,125.14$, $118.62(\mathrm{q}, J=322.2 \mathrm{~Hz}), 114.34,68.84,58.96,55.37,47.02,34.08$. HRMS (ESI) Calcd for $\left[\mathrm{C}_{20} \mathrm{H}_{25} \mathrm{BF}_{3} \mathrm{NNaO}_{4} \mathrm{~S}, \mathrm{M}+\mathrm{Na}\right]^{+}$: 466.1442; Found: 466.1447. 


\section{3-(benzyl(methyl)aminoborane)-2-(4-fluorobenzyl)prop-1-en-1-yl}

\section{trifluoromethanesulfonate $(\mathbf{4 q})$}<smiles>[BH3-]N(C/C(=C\[OH2+])Cc1ccc(F)cc1)Cc1ccccc1</smiles>

Z-4q: $26 \%$ yield for four steps, White solid, mp: $74-76$

${ }^{\circ} \mathrm{C} .{ }^{1} \mathrm{H}$ NMR $\left(400 \mathrm{MHz}, \mathrm{CDCl}_{3}\right) \delta 7.43-7.35(\mathrm{~m}, 3 \mathrm{H})$, $7.31-7.29(\mathrm{~m}, 2 \mathrm{H}), 7.18-7.15(\mathrm{~m}, 2 \mathrm{H}), 7.02-6.98(\mathrm{~m}, 2 \mathrm{H}), 6.71(\mathrm{~s}, 1 \mathrm{H}), 4.08-3.98$ (m, 3H), $3.68(\mathrm{~d}, J=15.6 \mathrm{~Hz}, 1 \mathrm{H}), 3.30(\mathrm{~d}, J=12.4 \mathrm{~Hz}, 1 \mathrm{H}), 3.06(\mathrm{~d}, J=12.0 \mathrm{~Hz}, 1 \mathrm{H})$, $2.54(\mathrm{~s}, 3 \mathrm{H}) .{ }^{13} \mathrm{C} \mathrm{NMR}\left(101 \mathrm{MHz}, \mathrm{CDCl}_{3}\right) \delta 162.06(\mathrm{~d}, J=245.7 \mathrm{~Hz}), 137.27,132.64$ $(\mathrm{d}, J=3.4 \mathrm{~Hz}), 132.61,130.94(\mathrm{~d}, J=8.1 \mathrm{~Hz}), 130.50,129.51,128.54,125.27,118.41$ $(\mathrm{q}, J=322.2 \mathrm{~Hz}), 115.82(\mathrm{~d}, J=21.4 \mathrm{~Hz}), 69.59,55.23,47.88,38.32$.

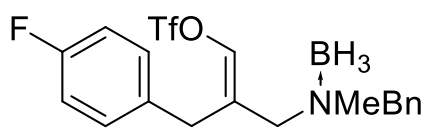

E-4q: $16 \%$ yield for four steps, white solid, mp: $85-87^{\circ} \mathrm{C}$. ${ }^{1} \mathrm{H}$ NMR (400 MHz, $\left.\mathrm{CDCl}_{3}\right) \delta 7.44-7.35$ (m, 3H), $7.28-$ $7.25(\mathrm{~m}, 2 \mathrm{H}), 7.13-7.08(\mathrm{~m}, 2 \mathrm{H}), 7.01-6.96(\mathrm{~m}, 2 \mathrm{H}), 6.64(\mathrm{~s}, 1 \mathrm{H}), 4.10-3.97(\mathrm{~m}$, $3 \mathrm{H}), 3.84(\mathrm{~d}, J=14.8 \mathrm{~Hz}, 1 \mathrm{H}), 3.23(\mathrm{~d}, J=12.8 \mathrm{~Hz}, 1 \mathrm{H}), 2.92(\mathrm{~d}, J=12.8 \mathrm{~Hz}, 1 \mathrm{H})$, $2.49(\mathrm{~s}, 3 \mathrm{H}) .{ }^{13} \mathrm{C} \mathrm{NMR}\left(101 \mathrm{MHz}, \mathrm{CDCl}_{3}\right) \delta 161.93(\mathrm{~d}, J=245.6 \mathrm{~Hz}), 138.32,132.83$ $(\mathrm{d}, J=3.2 \mathrm{~Hz}), 132.63,130.70(\mathrm{~d}, J=7.9 \mathrm{~Hz}), 130.42,129.55,128.71,124.72,118.60$ (q, $J=322 \mathrm{~Hz}), 115.81(\mathrm{~d}, J=21.3 \mathrm{~Hz}), 69.02,58.87,47.08,34.14$. HRMS (ESI) Calcd for $\left[\mathrm{C}_{19} \mathrm{H}_{22} \mathrm{BF}_{4} \mathrm{NNaO}_{3} \mathrm{~S}, \mathrm{M}+\mathrm{Na}\right]^{+}$: 454.1242; Found: 454.1246 .

\section{(Z)-3-(benzyl(methyl)aminoborane)-2-methylprop-1-en-1-yl}

\section{trifluoromethanesulfonate $(4 r)$}

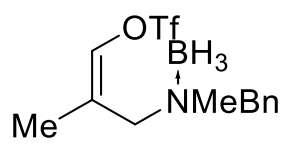

$35 \%$ yield for four steps. White solid, mp: $62-64{ }^{\circ} \mathrm{C} .{ }^{1} \mathrm{H}$ NMR $\left(400 \mathrm{MHz}, \mathrm{CDCl}_{3}\right) \delta 7.48-7.39(\mathrm{~m}, 3 \mathrm{H}), 7.39-7.28(\mathrm{~m}, 2 \mathrm{H}), 6.57$ (s, 1H), $4.07(\mathrm{~d}, J=13.3 \mathrm{~Hz}, 1 \mathrm{H}), 4.02(\mathrm{~d}, J=13.3 \mathrm{~Hz}, 1 \mathrm{H}), 3.37$ (d, $J=12.7 \mathrm{~Hz}, 1 \mathrm{H}), 3.14(\mathrm{~d}, J=12.7 \mathrm{~Hz}, 1 \mathrm{H}), 2.49$ (s, 3H), 2.04 (d, $J=1.4 \mathrm{~Hz}, 3 \mathrm{H})$. ${ }^{13} \mathrm{C} \mathrm{NMR}\left(101 \mathrm{MHz}, \mathrm{CDCl}_{3}\right) \delta 138.32,132.68,130.66,129.53,128.66,122.08,118.62$ $(\mathrm{q}, \quad J=322.2 \mathrm{~Hz}), 68.67,63.18$, 47.17, 16.91. HRMS (ESI) Calcd for $\left[\mathrm{C}_{13} \mathrm{H}_{19} \mathrm{BF}_{3} \mathrm{NNaO}_{3} \mathrm{~S}, \mathrm{M}+\mathrm{Na}\right]^{+}:$360.1023; Found: 360.1027. 


\section{(Z)-2-((benzyl(methyl)aminoborane)methyl)hex-1-en-1-yl}

\section{trifluoromethanesulfonate $(4 \mathrm{~s})$}

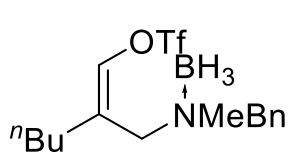

$26 \%$ yield for four steps. White solid, mp: $89-91{ }^{\circ} \mathrm{C} .{ }^{1} \mathrm{H}$ NMR $(400$ $\left.\mathrm{MHz}, \mathrm{CDCl}_{3}\right) \delta 7.48-7.39(\mathrm{~m}, 3 \mathrm{H}), 7.37(\mathrm{~d}, J=5.8 \mathrm{~Hz}, 2 \mathrm{H}), 6.55$ (s, 1H), $4.08(\mathrm{~d}, J=13.2 \mathrm{~Hz}, 1 \mathrm{H}), 4.01(\mathrm{~d}, J=13.3 \mathrm{~Hz}, 1 \mathrm{H}), 3.40$ $(\mathrm{d}, J=12.8 \mathrm{~Hz}, 1 \mathrm{H}), 3.11(\mathrm{~d}, J=12.8 \mathrm{~Hz}, 1 \mathrm{H}), 2.71-2.56(\mathrm{~m}, 1 \mathrm{H}), 2.47(\mathrm{~s}, 3 \mathrm{H}), 2.45$ - $2.36(\mathrm{~m}, 1 \mathrm{H}), 1.50-1.22(\mathrm{~m}, 4 \mathrm{H}), 0.91(\mathrm{t}, J=6.6 \mathrm{~Hz}, 3 \mathrm{H}) .{ }^{13} \mathrm{C}$ NMR (101 MHz, $\left.\mathrm{CDCl}_{3}\right) \delta 138.20,132.69,130.75,129.49,128.64,126.16,118.62(\mathrm{q}, J=322.2 \mathrm{~Hz})$, 68.52, 60.32, 46.88, 29.98, 29.30, 22.51, 13.83. HRMS (ESI) Calcd for $\left[\mathrm{C}_{16} \mathrm{H}_{25} \mathrm{BF}_{3} \mathrm{NNaO}_{3} \mathrm{~S}, \mathrm{M}+\mathrm{Na}\right]^{+}:$402.1493; Found: 402.1498.

\section{(Z)-2-((benzyl(methyl)aminoborane)methyl)-3-methylbut-1-en-1-yl}

\section{trifluoromethanesulfonate $(4 \mathrm{t})$}

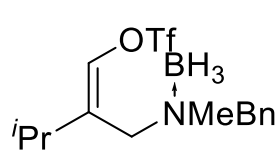

$29 \%$ yield for four steps. White solid, mp: $49-53{ }^{\circ} \mathrm{C} .{ }^{1} \mathrm{H}$ NMR $\left(400 \mathrm{MHz}, \mathrm{CDCl}_{3}\right) \delta 7.48-7.39(\mathrm{~m}, 3 \mathrm{H}), 7.39-7.28(\mathrm{~m}, 2 \mathrm{H})$, $6.58(\mathrm{~s}, 1 \mathrm{H}), 4.08(\mathrm{~d}, J=13.3 \mathrm{~Hz}, 1 \mathrm{H}), 4.02(\mathrm{~d}, J=13.3 \mathrm{~Hz}, 1 \mathrm{H})$, $3.36(\mathrm{~d}, J=12.8 \mathrm{~Hz}, 1 \mathrm{H}), 3.12(\mathrm{~d}, J=12.8 \mathrm{~Hz}, 1 \mathrm{H}), 2.89-2.78(\mathrm{~m}, 1 \mathrm{H}), 2.47(\mathrm{~s}, 3 \mathrm{H})$, $1.24(\mathrm{~d}, J=7.0 \mathrm{~Hz}, 3 \mathrm{H}), 1.16(\mathrm{~d}, J=7.1 \mathrm{~Hz}, 3 \mathrm{H}) .{ }^{13} \mathrm{C} \mathrm{NMR}\left(101 \mathrm{MHz}, \mathrm{CDCl}_{3}\right) \delta 138.48$, $132.72,130.72,129.72,129.56,128.70,118.61(\mathrm{q}, J=322.2 \mathrm{~Hz}), 68.63,62.34,46.80$, 32.25, 20.71, 20.19. HRMS (ESI) Calcd for $\left[\mathrm{C}_{15} \mathrm{H}_{23} \mathrm{BF}_{3} \mathrm{NNaO}_{3} \mathrm{~S}, \mathrm{M}+\mathrm{Na}\right]^{+}$: 388.1336; Found: 388.1341.

\section{(E)-3-(benzyl(methyl)aminoborane)-2-cyclohexylprop-1-en-1-yl}

\section{trifluoromethanesulfonate $(4 \mathrm{u})$}

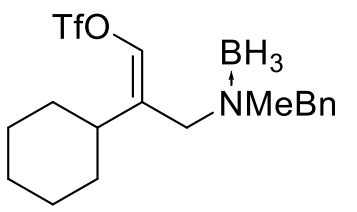

$54 \%$ yield for four steps. Colorless oil. ${ }^{1} \mathrm{H}$ NMR (400 MHz, $\left.\mathrm{CDCl}_{3}\right) \delta 7.46-7.39(\mathrm{~m}, 3 \mathrm{H}), 7.39-7.34(\mathrm{~m}, 2 \mathrm{H}), 6.62(\mathrm{~s}, 1 \mathrm{H})$, $4.08(\mathrm{~d}, J=13.1 \mathrm{~Hz}, 1 \mathrm{H}), 4.02(\mathrm{~d}, J=13.3 \mathrm{~Hz}, 1 \mathrm{H}), 3.48(\mathrm{~d}, J=$

$12.1 \mathrm{~Hz}, 1 \mathrm{H}), 3.27(\mathrm{~d}, J=12.2 \mathrm{~Hz}, 1 \mathrm{H}), 2.78(\mathrm{t}, J=11.8 \mathrm{~Hz}, 1 \mathrm{H}), 2.48(\mathrm{~s}, 3 \mathrm{H}), 1.95(\mathrm{~d}$, $J=11.5 \mathrm{~Hz}, 2 \mathrm{H}), 1.81-1.70(\mathrm{~m}, 3 \mathrm{H}), 1.37-1.26(\mathrm{~m}, 2 \mathrm{H}), 1.24-1.03(\mathrm{~m}, 2 \mathrm{H}), 0.99$ $-0.79(\mathrm{~m}, 1 \mathrm{H}) .{ }^{13} \mathrm{C} \mathrm{NMR}\left(101 \mathrm{MHz}, \mathrm{CDCl}_{3}\right) \delta 137.39,132.63,131.17,130.77,129.45$, 
$128.51,118.42$ (q, $J=322.2 \mathrm{~Hz}), 69.24,56.53,47.19,41.68,34.53,31.23,26.75,26.58$, 26.13. HRMS (ESI) Calcd for $\left[\mathrm{C}_{18} \mathrm{H}_{27} \mathrm{BF}_{3} \mathrm{NNaO}_{3} \mathrm{~S}, \mathrm{M}+\mathrm{Na}\right]^{+}$: 428.1649; Found: 428.1653.

(E)-3-(dimethylaminoborane)-2-phenylprop-1-en-1-yl trifluoromethanesulfonate (4v)<smiles>CN(C)CC(=CO)c1ccccc1</smiles>
$61 \%$ yield for four steps. White solid, mp: $69-72{ }^{\circ} \mathrm{C} .{ }^{1} \mathrm{H}$ NMR (400 $\left.\mathrm{MHz}, \mathrm{CDCl}_{3}\right) \delta 7.52-7.38(\mathrm{~m}, 3 \mathrm{H}), 7.37-7.29(\mathrm{~m}, 2 \mathrm{H}), 6.94(\mathrm{~s}$,

$1 \mathrm{H}), 3.86(\mathrm{~s}, 2 \mathrm{H}), 2.39(\mathrm{~s}, 6 \mathrm{H}) .{ }^{13} \mathrm{C} \mathrm{NMR}\left(101 \mathrm{MHz}, \mathrm{CDCl}_{3}\right) \delta 137.62$, 133.10, 129.43, 129.27, 128.05, 124.49, 118.53 (q, $J=322.2 \mathrm{~Hz}), 64.56,51.20$. HRMS (ESI) Calcd for $\left[\mathrm{C}_{12} \mathrm{H}_{17} \mathrm{BF}_{3} \mathrm{NNaO}_{3} \mathrm{~S}, \mathrm{M}+\mathrm{Na}\right]^{+}: 346.0867$; Found: 346.0870 .

\section{(E)-3-(dimethylaminoborane)-2-(4-methoxyphenyl)prop-1-en-1-yl} trifluoromethanesulfonate $(4 \mathrm{w})$

$67 \%$ yield for four steps. White solid, mp: $101-103{ }^{\circ} \mathrm{C} .{ }^{1} \mathrm{H}$
$\mathrm{N}=8.4 \mathrm{~Hz}, 2 \mathrm{H}), 6.89(\mathrm{~s}, 1 \mathrm{H}), 3.84(\mathrm{~s}, 3 \mathrm{H}), 3.83(\mathrm{~s}, 2 \mathrm{H}), 2.39$ $(\mathrm{s}, 6 \mathrm{H}) .{ }^{13} \mathrm{C} \mathrm{NMR}\left(101 \mathrm{MHz}, \mathrm{CDCl}_{3}\right) \delta 160.24,136.96,129.38,124.96,124.15,118.51$ $(\mathrm{q}, \quad J=322.2 \mathrm{~Hz}), 114.64,64.36,55.43,51.10$. HRMS (ESI) Calcd for $\left[\mathrm{C}_{13} \mathrm{H}_{19} \mathrm{BF}_{3} \mathrm{NNaO}_{4} \mathrm{~S}, \mathrm{M}+\mathrm{Na}\right]^{+}$: 376.0972; Found: 376.0976.

\section{(E)-2-(4-chlorophenyl)-3-(dimethylaminoborane)prop-1-en-1-yl}

trifluoromethanesulfonate $(4 x)$

$60 \%$ yield for four steps. White solid, mp: $106-108{ }^{\circ} \mathrm{C} .{ }^{1} \mathrm{H}$
$=8.8 \mathrm{~Hz}, 2 \mathrm{H}), 6.96(\mathrm{~s}, 1 \mathrm{H}), 3.82(\mathrm{~s}, 2 \mathrm{H}), 2.41(\mathrm{~s}, 6 \mathrm{H}) .{ }^{13} \mathrm{C}$ NMR (101 MHz, $\left.\mathrm{CDCl}_{3}\right) \delta 138.05,135.44,131.52,129.53,129.37,123.13,118.43$ (q, $J=322.2 \mathrm{~Hz}), 64.40,51.38$. HRMS (ESI) Calcd for $\left[\mathrm{C}_{12} \mathrm{H}_{16} \mathrm{BClF}_{3} \mathrm{NNaO}_{3} \mathrm{~S}, \mathrm{M}+\mathrm{Na}\right]^{+}$: 380.0477; Found: 380.0480 . 
(Z)-3-(dimethylaminoborane)-2-(4-(trifluoromethyl)phenyl)prop-1-en-1-yl

trifluoromethanesulfonate $(4 y)$

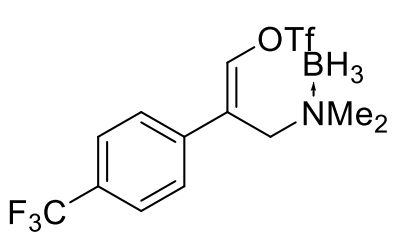

$31 \%$ yield for four steps. White solid, mp: $65-67{ }^{\circ} \mathrm{C} .{ }^{1} \mathrm{H}$ NMR (400 MHz, $\left.\mathrm{CDCl}_{3}\right) \delta 7.71(\mathrm{~d}, J=8.0 \mathrm{~Hz}, 2 \mathrm{H}), 7.50$ (d, $J=8.4 \mathrm{~Hz}, 2 \mathrm{H}), 7.09(\mathrm{~s}, 1 \mathrm{H}), 4.09(\mathrm{~s}, 2 \mathrm{H}), 2.47(\mathrm{~s}, 6 \mathrm{H}) .{ }^{13} \mathrm{C}$ NMR $\left(101 \mathrm{MHz}, \mathrm{CDCl}_{3}\right) \delta 139.55,139.24(\mathrm{q}, J=1.3 \mathrm{~Hz})$,

$131.58(\mathrm{q}, J=33.1 \mathrm{~Hz}), 127.48,126.58(\mathrm{q}, J=3.7 \mathrm{~Hz}), 125.09,123.61,122.39,118.52$ (q, $J=322.2 \mathrm{~Hz}$ ), 58.75, 51.62. HRMS (ESI) Calcd for $\left[\mathrm{C}_{13} \mathrm{H}_{16} \mathrm{BF}_{6} \mathrm{NNaO}_{3} \mathrm{~S}, \mathrm{M}+\mathrm{Na}\right]^{+}$: 414.0740; Found: 414.0744. E-4y was too little to be separated. 


\section{Typical Procedures of B-H Bond Insertion Reactions and Additional Studies on Reaction Conditions}

\subsection{Intermolecular}<smiles>C[13CH2]OC=C1CCC(c2ccccc2)CC1</smiles>

Under an argon atmosphere, trimethylamine-borane adduct $\mathbf{2 a}(87.5 \mathrm{mg}, 1.2 \mathrm{mmol})$ and ${ }^{t} \mathrm{BuOK}$ (40.4 mg, $\left.0.36 \mathrm{mmol}\right)$ were introduced into a Schlenk tube. An ice bath was used to cool the reaction system to $0^{\circ} \mathrm{C}$. Then $\mathrm{CH}_{2} \mathrm{Cl}_{2}(3 \mathrm{~mL})$ was injected into the Schlenk tube in one portion. Alkenyl triflate 1a $(96.1 \mathrm{mg}, 0.3 \mathrm{mmol})$ was then added into this reaction mixture in one portion. The resulting mixture was stirred at $0{ }^{\circ} \mathrm{C}$ for 2 h. The product was purified by flash column chromatography on silica gel (petroleum ether, PE/ethyl acetate, $\left.\mathrm{EA} / \mathrm{Et}_{3} \mathrm{~N}=15: 1: 0.05, v / v / v\right)$.

\subsection{Intramolecular}

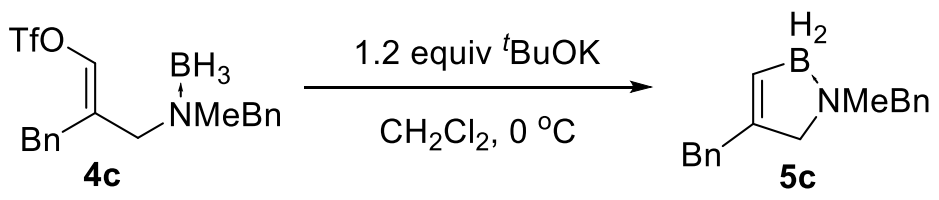

Under an argon atmosphere, ${ }^{t} \mathrm{BuOK}(40.4 \mathrm{mg}, 0.36 \mathrm{mmol})$ was introduced into a $25 \mathrm{~mL}$ Schlenk tube. An ice bath was used to cool the reaction system to $0^{\circ} \mathrm{C}$. Then $2 \mathrm{~mL}$ $\mathrm{CH}_{2} \mathrm{Cl}_{2}$ was injected into the Schlenk tube in one portion. To this reaction mixture a solution of $4 \mathbf{c}(124.0 \mathrm{mg}, 0.3 \mathrm{mmol})$ in $1 \mathrm{~mL}$ of $\mathrm{CH}_{2} \mathrm{Cl}_{2}$ was then added in one portion. The resulting mixture was stirred at $0{ }^{\circ} \mathrm{C}$ for $2 \mathrm{~h}$. The product was purified by flash column chromatography on silica gel $\left(\mathrm{PE} / \mathrm{EA} / \mathrm{Et}_{3} \mathrm{~N}=15: 1: 0.05, v / v / v\right)$. 


\subsection{Temperature effect}

Table S1. Studies on temperature effect ${ }^{a}$

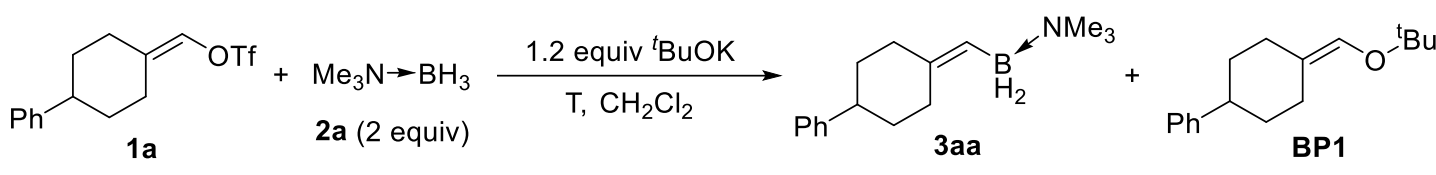

\begin{tabular}{llllll}
\hline entry & $\mathrm{T}\left({ }^{\circ} \mathrm{C}\right)$ & time $(\mathrm{h})$ & conv. $(\%)$ & yield $(\%)^{b}$ & BP1 $(\%)^{c}$ \\
\hline 1 & 0 & 2 & $\sim 95$ & 70 & $\sim 5$ \\
2 & $\operatorname{rt}(20)$ & 2 & $\sim 95$ & 66 & $\sim 5$ \\
3 & 40 & 2 & $\sim 85$ & 55 & $\sim 5$ \\
4 & -40 & 10 & $\sim 40$ & $\sim 35$ & $\sim 4$ \\
\hline
\end{tabular}

${ }^{a}$ Reaction conditions: $1 \mathbf{a}(0.3 \mathrm{mmol}), \mathbf{2 a}(1.2 \mathrm{mmol}),{ }^{t} \mathrm{BuOK}(0.36 \mathrm{mmol}), 3 \mathrm{~mL} \mathrm{CH}_{2} \mathrm{Cl}_{2}$, $0{ }^{\circ} \mathrm{C} .{ }^{b}$ Isolated yield. ${ }^{c}$ Determined by ${ }^{1} \mathrm{H}$ NMR using 1,3,5-trimethoxybenzene as an internal standard.

\subsection{Byproducts of the reactions with $2 \mathrm{~d}-2 \mathrm{f}$}

Table S2. Byproducts of the reactions with $2 \mathrm{~d}-2 \mathrm{f}^{a}$

\begin{tabular}{|c|c|c|c|c|c|c|}
\hline entry & $X$ & $\begin{array}{l}\text { conv. } \\
(\%)^{b}\end{array}$ & $\begin{array}{c}\text { yield of } 3 \\
(\%)^{b}\end{array}$ & $\begin{array}{c}\text { yield of } \\
\text { BP1 }(\%)^{b}\end{array}$ & $\begin{array}{c}\text { yield of } \\
\text { BP2 }(\%)^{b}\end{array}$ & $\begin{array}{c}\text { yield of } \\
\text { BP3 }(\%)^{b}\end{array}$ \\
\hline 1 & $\begin{array}{c}{ }^{n} \mathrm{Bu}_{3} \mathrm{~N} \\
(\mathbf{2 d})\end{array}$ & $>95$ & $<5$ & $\sim 30$ & $\sim 20$ & N.D. ${ }^{c}$ \\
\hline 2 & $\begin{array}{c}\mathrm{Me}_{2} \mathrm{NH} \\
(\mathbf{2 e})\end{array}$ & $\sim 10$ & N.D. & N.D. & $\sim 5$ & $\sim 65$ \\
\hline 3 & $\begin{array}{c}\text { Pyridine } \\
(\mathbf{2 f})\end{array}$ & $\sim 90 \%$ & N.D. & $\sim 35$ & $\sim 25$ & N.D. \\
\hline
\end{tabular}

${ }^{a}$ Reaction conditions: 1a $(0.3 \mathrm{mmol}), 2(1.2 \mathrm{mmol}),{ }^{t} \mathrm{BuOK}(0.36 \mathrm{mmol}), 3 \mathrm{~mL} \mathrm{CH}_{2} \mathrm{Cl}_{2}$, $0{ }^{\circ} \mathrm{C} .{ }^{b}$ Determined by ${ }^{1} \mathrm{H}$ NMR using 1,3,5-trimethoxybenzene as an internal standard. ${ }^{c}$ Not detected. 


\section{Analytical Data of Insertion Products}

(4-(trimethylamine-boranylmethylene)cyclohexyl)benzene (3aa)

$77 \%$ yield. White solid, mp: $51-54{ }^{\circ} \mathrm{C} .{ }^{1} \mathrm{H} \mathrm{NMR}(400 \mathrm{MHz}$,
$\left.\mathrm{CDCl}_{3}\right) \delta 7.30-7.25(\mathrm{~m}, 2 \mathrm{H}), 7.23-7.14(\mathrm{~m}, 3 \mathrm{H}), 5.41(\mathrm{t}$, $J=5.9 \mathrm{~Hz}, 1 \mathrm{H}), 3.15(\mathrm{dt}, J=13.3,2.4 \mathrm{~Hz}, 1 \mathrm{H}), 2.69$ (tt, $J=12.2,3.4 \mathrm{~Hz}, 1 \mathrm{H}), 2.55$ (s, 9H), $2.43-2.34(\mathrm{~m}, 2 \mathrm{H}), 2.03-1.89(\mathrm{~m}, 3 \mathrm{H}), 1.67-1.45(\mathrm{~m}, 2 \mathrm{H}) .{ }^{13} \mathrm{C}$ NMR $(101$ $\left.\mathrm{MHz}, \mathrm{CDCl}_{3}\right) \delta 148.83,147.86,128.33,127.01,125.82,51.80,45.15,40.71,36.59$, 35.95, 31.32. HRMS (ESI) Calcd for $\left[\mathrm{C}_{16} \mathrm{H}_{27} \mathrm{BN}, \mathrm{M}+\mathrm{H}\right]^{+}: 244.2231$; Found: 244.2236.

\section{(4-(tributylphosphane-boranylmethylene)cyclohexyl)benzene (3ab)}

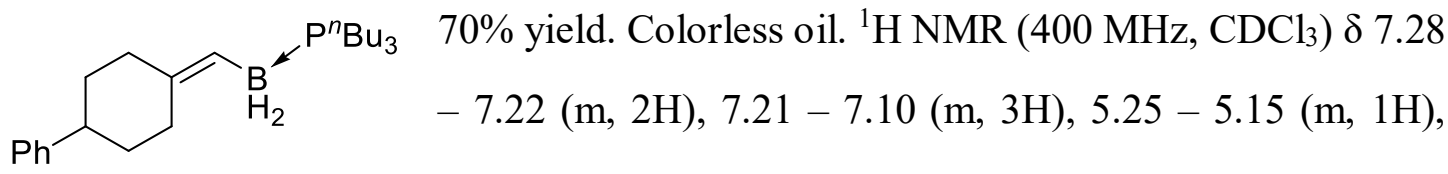
$3.15-3.04(\mathrm{~m}, 1 \mathrm{H}), 2.72-2.60(\mathrm{~m}, 1 \mathrm{H}), 2.39-2.22(\mathrm{~m}, 2 \mathrm{H})$, $1.99-1.80(\mathrm{~m}, 3 \mathrm{H}), 1.64-1.30(\mathrm{~m}, 20 \mathrm{H}), 1.00-0.84(\mathrm{~m}, 9 \mathrm{H}) .{ }^{13} \mathrm{C}$ NMR $(101 \mathrm{MHz}$, $\left.\mathrm{CDCl}_{3}\right) \delta 147.74,146.50(\mathrm{~d}, J=16.1 \mathrm{~Hz}), 128.09,126.75,125.55,45.08,40.36(\mathrm{~d}, J=$ $3.3 \mathrm{~Hz}), 36.47(\mathrm{~d}, J=4.0 \mathrm{~Hz}), 35.68(\mathrm{~d}, J=2.9 \mathrm{~Hz}), 31.24,27.82,24.61(\mathrm{~d}, J=2.2 \mathrm{~Hz})$, 24.55 (d, $J=2.7 \mathrm{~Hz}), 24.49,24.36,24.23,22.89,22.55,20.93$ (d, $J=31.8 \mathrm{~Hz}), 13.57$. ${ }^{11} \mathrm{~B}$ NMR $\left(128 \mathrm{MHz}, \mathrm{CDCl}_{3}\right) \delta-32.21$. HRMS (ESI) Calcd for $\left[\mathrm{C}_{25} \mathrm{H}_{44} \mathrm{BNaP}, \mathrm{M}+\mathrm{Na}\right]^{+}$: 409.3166; Found: 409.3170.

\section{(1,3-dimethyl-1H-imidazol-3-ium-2-yl)((4-}

phenylcyclohexylidene)methyl)dihydroborate (3ac)

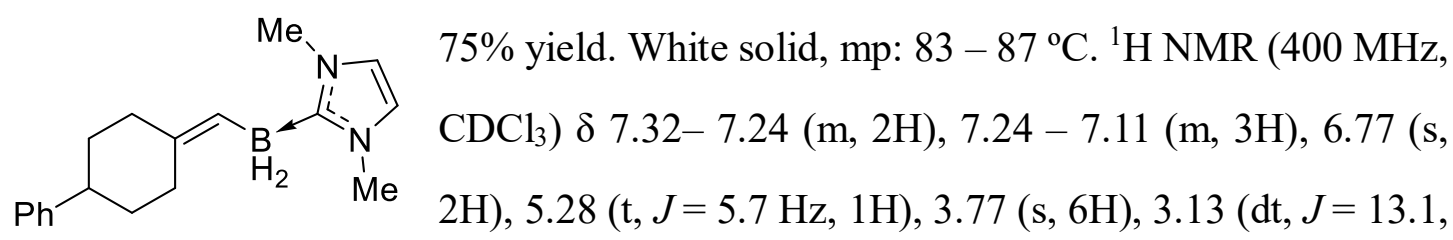
$3.8 \mathrm{~Hz}, 1 \mathrm{H}), 2.67(\mathrm{tt}, J=12.1,3.3 \mathrm{~Hz}, 1 \mathrm{H}), 2.37-2.25(\mathrm{~m}, 2 \mathrm{H}), 1.98-1.86(\mathrm{~m}, 3 \mathrm{H})$, 1.61-1.35 (m, 2H). ${ }^{13} \mathrm{C}$ NMR (101 MHz, $\left.\mathrm{CDCl}_{3}\right) \delta 148.05,143.76,128.25,126.96$, $125.69,120.12,45.21,40.40,36.49,36.01,35.95,31.24 .{ }^{11} \mathrm{~B}$ NMR (128 MHz, $\left.\mathrm{CDCl}_{3}\right)$ 
$\delta$-30.02 (t, $J=83.5 \mathrm{~Hz})$. HRMS (ESI) Calcd for $\left[\mathrm{C}_{18} \mathrm{H}_{25} \mathrm{BN}_{2} \mathrm{Na}, \mathrm{M}+\mathrm{Na}\right]^{+}$: 303.2003 ; Found: 303.2005.

\section{(trimethylamine-boranylmethylene)cyclopentane (3ba)}

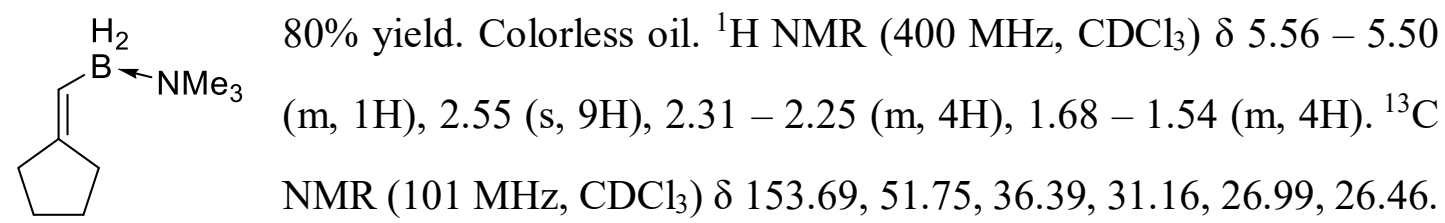

HRMS (ESI) Calcd for $\left[\mathrm{C}_{9} \mathrm{H}_{21} \mathrm{BN}, \mathrm{M}+\mathrm{H}\right]^{+}$: 154.1762; Found: 154.1751.

\section{(trimethylamine-boranylmethylene)cyclohexane (3ca)}

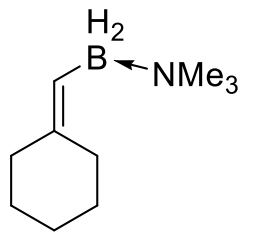

$81 \%$ yield. Colorless oil. ${ }^{1} \mathrm{H}$ NMR $\left(400 \mathrm{MHz}, \mathrm{CDCl}_{3}\right) \delta 5.31$ (t, $J=6.0$

$\mathrm{Hz}, 1 \mathrm{H}), 2.54(\mathrm{~s}, 9 \mathrm{H}), 2.35(\mathrm{t}, J=5.8 \mathrm{~Hz}, 2 \mathrm{H}), 2.17(\mathrm{t}, J=5.8 \mathrm{~Hz}, 2 \mathrm{H})$, $1.55-1.52(\mathrm{~m}, 4 \mathrm{H}), 1.49-1.45(\mathrm{~m}, 2 \mathrm{H}) .{ }^{13} \mathrm{C} \mathrm{NMR}\left(101 \mathrm{MHz}, \mathrm{CDCl}_{3}\right)$

$\delta 150.42,51.76,41.12,31.68,29.43,28.60,27.36 . H R M S$ (ESI) Calcd for $\left[\mathrm{C}_{10} \mathrm{H}_{23} \mathrm{BN}, \mathrm{M}+\mathrm{H}\right]^{+}$: 168.1918; Found: 168.1919 .

(trimethylamine-boranylmethylene) cycloheptane (3da)

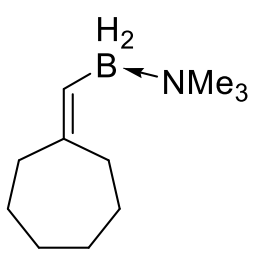

$73 \%$ yield. Colorless oil. ${ }^{1} \mathrm{H}$ NMR (400 $\left.\mathrm{MHz}, \mathrm{CDCl}_{3}\right) \delta 5.39$ (t, $J=$ $5.5 \mathrm{~Hz}, 1 \mathrm{H}), 2.55(\mathrm{~s}, 9 \mathrm{H}), 2.41(\mathrm{t}, J=6.1 \mathrm{~Hz}, 2 \mathrm{H}), 2.30(\mathrm{t}, J=6.1 \mathrm{~Hz}$, 2H), $1.58-1.54(\mathrm{~m}, 4 \mathrm{H}), 1.52-1.48(\mathrm{~m}, 4 \mathrm{H}) .{ }^{13} \mathrm{C}$ NMR $(101 \mathrm{MHz}$, $\left.\mathrm{CDCl}_{3}\right) \delta 150.88,51.79,41.47,32.60,29.95,29.54,28.09$. HRMS (ESI) Calcd for $\left[\mathrm{C}_{11} \mathrm{H}_{24} \mathrm{BNNa}, \mathrm{M}+\mathrm{Na}\right]^{+}$: 204.1894; Found: 204.1896.

\section{1-(trimethylamine-boranyl)-2-methylprop-1-ene (3ea)}<smiles>C[B]C=C(C)C</smiles>

$63 \%$ yield. Colorless oil. ${ }^{1} \mathrm{H}$ NMR (400 MHz, $\left.\mathrm{CDCl}_{3}\right) \delta 5.50-5.34$ (m, 1H), $2.54(\mathrm{~s}, 9 \mathrm{H}), 1.81(\mathrm{~s}, 3 \mathrm{H}), 1.76(\mathrm{~s}, 3 \mathrm{H}) .{ }^{13} \mathrm{C} \mathrm{NMR}(101 \mathrm{MHz}$, $\left.\mathrm{CDCl}_{3}\right) \delta 141.68,51.72,29.28,20.66$. HRMS (ESI) Calcd for $\left[\mathrm{C}_{17} \mathrm{H}_{18} \mathrm{BNNa}, \mathrm{M}+\mathrm{Na}\right]^{+}:$150.1425; Found: 150.1432. 


\section{3-(trimethylamine-boranylmethylene)pentane (3fa)}<smiles>CCC(=CB[NH3+])CC</smiles>

$79 \%$ yield. Colorless oil. ${ }^{1} \mathrm{H}$ NMR $\left(400 \mathrm{MHz}, \mathrm{CDCl}_{3}\right) \delta 5.32(\mathrm{t}, J=$ $5.7 \mathrm{~Hz}, 1 \mathrm{H}), 2.52(\mathrm{~s}, 9 \mathrm{H}), 2.21(\mathrm{q}, J=7.5 \mathrm{~Hz}, 2 \mathrm{H}), 2.05(\mathrm{q}, J=7.4$ $\mathrm{Hz}, 2 \mathrm{H}), 0.99$ (t, $J=7.5 \mathrm{~Hz}, 3 \mathrm{H}), 0.93$ (t, $J=7.5 \mathrm{~Hz}, 3 \mathrm{H}) .{ }^{13} \mathrm{C} \mathrm{NMR}$ $\left(101 \mathrm{MHz}, \mathrm{CDCl}_{3}\right) \delta 152.71,51.74,31.39,25.89,13.89,13.36$. HRMS (ESI) Calcd for $\left[\mathrm{C}_{9} \mathrm{H}_{21} \mathrm{BN}, \mathrm{M}-\mathrm{H}\right]^{+}$: 154.1762; Found: 154.1748.

\section{7-(trimethylamine-boranylmethylene)tridecane (3ga)}

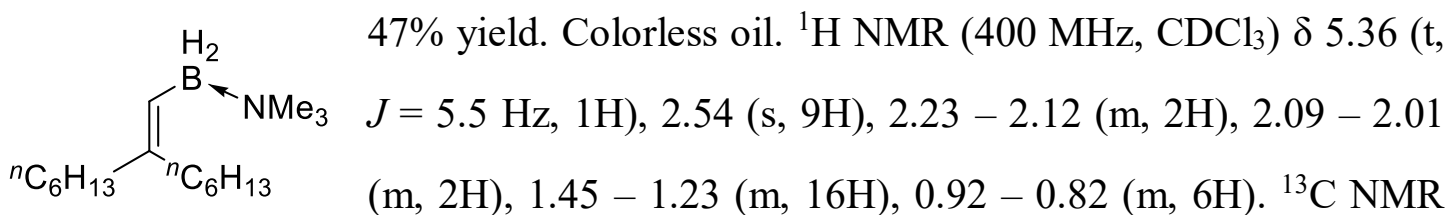
$\left(101 \mathrm{MHz}, \mathrm{CDCl}_{3}\right) \delta 150.18,51.84,39.80,33.08,32.15,32.09,30.00,29.59,29.49$, 28.92, 22.90, 14.31. HRMS (ESI) Calcd for $\left[\mathrm{C}_{17} \mathrm{H}_{39} \mathrm{BN}, \mathrm{M}+\mathrm{H}\right]^{+}:$268.3170; Found: 268.3179 .

\section{1-(3-(trimethylamine-boranyl)-2-methylallyl)-4-(tert-butyl)benzene (3ha)}

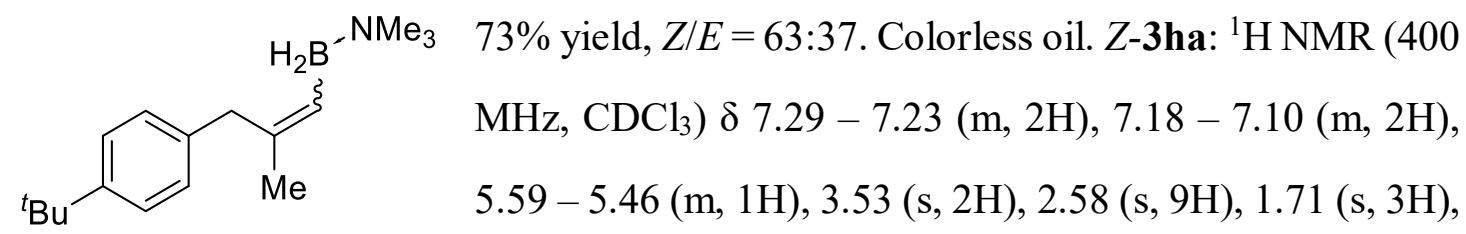
1.29 (s, 9H). ${ }^{13} \mathrm{C}$ NMR (101 MHz, $\left.\mathrm{CDCl}_{3}\right) \delta 147.86,143.52,139.40,128.46,124.93$, 51.93, 40.26, 34.34, 31.55, 26.53. E-3ha: ${ }^{1} \mathrm{H}$ NMR (400 MHz, $\left.\mathrm{CDCl}_{3}\right) \delta 7.29-7.23$ (m, 2H), $7.18-7.10(\mathrm{~m}, 2 \mathrm{H}), 5.59-5.46(\mathrm{~m}, 1 \mathrm{H}), 3.36(\mathrm{~s}, 2 \mathrm{H}), 2.54(\mathrm{~s}, 9 \mathrm{H}), 1.70(\mathrm{~s}$, $3 \mathrm{H}), 1.30(\mathrm{~s}, 9 \mathrm{H}) .{ }^{13} \mathrm{C}$ NMR $\left(101 \mathrm{MHz}, \mathrm{CDCl}_{3}\right) \delta 148.19,144.50,138.80,128.52$, $124.96,51.80,49.40,34.39,31.55,18.76$. The configuration of 3ha was inferred by that of 4,4,5,5-Tetramethyl-2-(2-methyl-3-phenylprop-1-en-1-yl)-1,3,2-dioxaborolane ${ }^{5}$. HRMS (ESI) Calcd for $\left[\mathrm{C}_{17} \mathrm{H}_{30} \mathrm{BNNa}, \mathrm{M}+\mathrm{Na}\right]^{+}: 282.2364$; Found: 282.2368.

\section{4-benzyl-1,1-dimethyl-2,5-dihydro-1 $H$-1 $\lambda^{4}, 2$-azaborole (5a)}

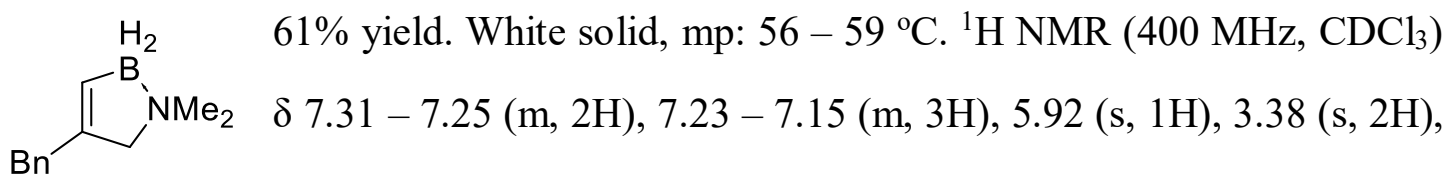


$3.30(\mathrm{~s}, 2 \mathrm{H}), 2.63(\mathrm{~s}, 6 \mathrm{H}) .{ }^{13} \mathrm{C} \mathrm{NMR}\left(101 \mathrm{MHz}, \mathrm{CDCl}_{3}\right) \delta 139.78,135.39,128.77$, 128.45, 126.09, 71.07, 51.79, 39.55. HRMS (ESI) Calcd for $\left[\mathrm{C}_{12} \mathrm{H}_{17} \mathrm{BN}, \mathrm{M}-\mathrm{H}\right]^{+}$: 186.1449; Found: 186.1454.

\section{4-benzyl-1-butyl-1-methyl-2,5-dihydro- $1 H$-1 $\lambda^{4}, 2$-azaborole (5b)}

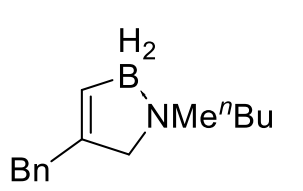

$73 \%$ yield. Colorless oil. ${ }^{1} \mathrm{H}$ NMR $\left(400 \mathrm{MHz}, \mathrm{CDCl}_{3}\right) \delta 7.31-7.23$ $(\mathrm{m}, 2 \mathrm{H}), 7.22-7.14(\mathrm{~m}, 3 \mathrm{H}), 5.91(\mathrm{~s}, 1 \mathrm{H}), 3.48-3.30(\mathrm{~m}, 3 \mathrm{H}), 3.07$ $(\mathrm{d}, J=13.9 \mathrm{~Hz}, 1 \mathrm{H}), 2.89-2.72(\mathrm{~m}, 2 \mathrm{H}), 2.50(\mathrm{~s}, 3 \mathrm{H}), 1.71-1.56$ (m, 1H), $1.51-1.39(\mathrm{~m}, 1 \mathrm{H}), 1.33-1.18(\mathrm{~m}, 2 \mathrm{H}), 0.90(\mathrm{dd}, J=7.8,6.9 \mathrm{~Hz}, 3 \mathrm{H}) .{ }^{13} \mathrm{C}$ NMR (101 MHz, $\left.\mathrm{CDCl}_{3}\right) \delta 139.83,134.91,128.75,128.39,126.01,69.35,62.98,48.40$, 39.55, 26.73, 20.55, 13.86. HRMS (ESI) Calcd for $\left[\mathrm{C}_{15} \mathrm{H}_{24} \mathrm{BNNa}, \mathrm{M}+\mathrm{Na}\right]^{+}$: 252.1894; Found: 252.1897.

\section{1,4-dibenzyl-1-methyl-2,5-dihydro- $1 H$-1 $1 \lambda^{4}, 2$-azaborole (5c)}<smiles></smiles>
75\% yield. White solid, mp: $98-103{ }^{\circ} \mathrm{C} .{ }^{1} \mathrm{H} \mathrm{NMR}\left(400 \mathrm{MHz}, \mathrm{CDCl}_{3}\right)$ $\delta 7.35-7.18(\mathrm{~m}, 8 \mathrm{H}), 7.03(\mathrm{~d}, J=7.0 \mathrm{~Hz}, 2 \mathrm{H}), 6.02(\mathrm{~s}, 1 \mathrm{H}), 3.97(\mathrm{dd}$, $J=39.8,13.4 \mathrm{~Hz}, 2 \mathrm{H}), 3.58(\mathrm{~d}, J=13.9 \mathrm{~Hz}, 1 \mathrm{H}), 3.39(\mathrm{dd}, J=27.8$, $14.6 \mathrm{~Hz}, 2 \mathrm{H}), 2.93(\mathrm{~d}, J=13.9 \mathrm{~Hz}, 1 \mathrm{H}), 2.45$ (s, 3H). ${ }^{13} \mathrm{C} \mathrm{NMR}\left(101 \mathrm{MHz}, \mathrm{CDCl}_{3}\right) \delta$ $139.91,135.45,132.11,131.85,128.95,128.88,128.55,128.51,126.14,66.28,65.04$, 47.39, 39.74. HRMS (ESI) Calcd for $\left[\mathrm{C}_{18} \mathrm{H}_{22} \mathrm{BNNa}, \mathrm{M}+\mathrm{Na}\right]^{+}$: 286.1738; Found: 286.1740 .

\section{7-benzyl-4 $\lambda^{4}$-aza-5-boraspiro[3.4]oct-6-ene (5d)}

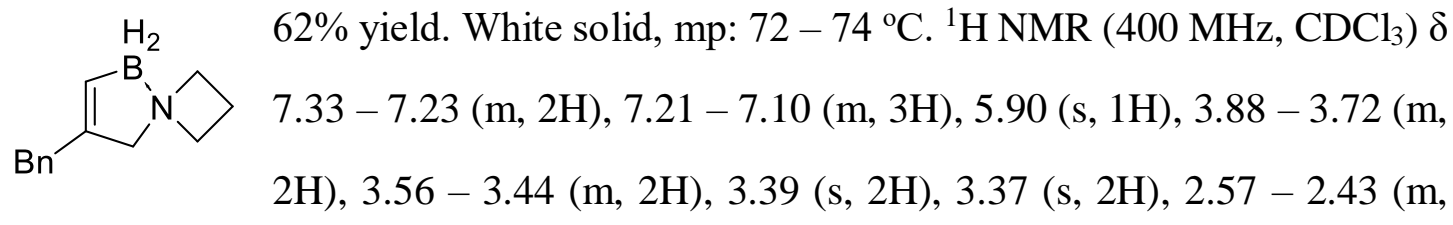

1H), $2.11-1.98(\mathrm{~m}, 1 \mathrm{H}) .{ }^{13} \mathrm{C}$ NMR $\left(101 \mathrm{MHz}, \mathrm{CDCl}_{3}\right) \delta$ 139.97, 136.05, 128.70, 128.41, 126.04, 70.48, 60.61, 39.48, 15.74. HRMS (ESI) Calcd for $\left[\mathrm{C}_{13} \mathrm{H}_{18} \mathrm{BNNa}, \mathrm{M}+\right.$ $\mathrm{Na}^{+}:$222.1425; Found: 222.1445. 


\section{3-benzyl-5 $\lambda^{4}$-aza-1-boraspiro[4.4]non-2-ene (5e)}

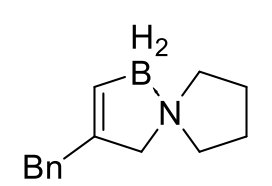

$86 \%$ yield from $Z-4 \mathbf{e}, 83 \%$ yield from $E$-4e. Colorless oil. ${ }^{1} \mathrm{H}$ NMR $\left(400 \mathrm{MHz}, \mathrm{CDCl}_{3}\right) \delta 7.31-7.23(\mathrm{~m}, 2 \mathrm{H}), 7.22-7.15(\mathrm{~m}, 3 \mathrm{H}), 5.91(\mathrm{~s}$,

$1 \mathrm{H}), 3.40(\mathrm{~s}, 2 \mathrm{H}), 3.38(\mathrm{~s}, 2 \mathrm{H}), 3.31-3.22(\mathrm{~m}, 2 \mathrm{H}), 2.75-2.67(\mathrm{~m}$, 2H), $2.14-2.00(\mathrm{~m}, 2 \mathrm{H}), 1.91-1.78(\mathrm{~m}, 2 \mathrm{H}) .{ }^{13} \mathrm{C} \mathrm{NMR}\left(101 \mathrm{MHz}, \mathrm{CDCl}_{3}\right) \delta 139.95$, 135.77, 128.74, 128.41, 126.02, 68.24, 61.20, 39.53, 22.47. HRMS (ESI) Calcd for $\left[\mathrm{C}_{14} \mathrm{H}_{19} \mathrm{BN}, \mathrm{M}-\mathrm{H}\right]^{+}:$212.1605; Found: 212.1611.

\section{3-benzyl-5 $\lambda^{4}$-aza-1-boraspiro[4.5]dec-2-ene (5f)}<smiles>BrC1=CBN2CCCCC12</smiles>

$76 \%$ yield. White solid, mp: $54-56{ }^{\circ} \mathrm{C} .{ }^{1} \mathrm{H} \mathrm{NMR}\left(400 \mathrm{MHz}, \mathrm{CDCl}_{3}\right)$ $\delta 7.32-7.24(\mathrm{~m}, 2 \mathrm{H}), 7.19(\mathrm{~d}, J=6.8 \mathrm{~Hz}, 3 \mathrm{H}), 5.89(\mathrm{~s}, 1 \mathrm{H}), 3.38(\mathrm{~s}$, 2H), $3.31(\mathrm{~s}, 2 \mathrm{H}), 3.12-3.01(\mathrm{~m}, 2 \mathrm{H}), 2.72-2.63(\mathrm{~m}, 2 \mathrm{H}), 1.94-$ $1.80(\mathrm{~m}, 2 \mathrm{H}), 1.61-1.38(\mathrm{~m}, 4 \mathrm{H}) .{ }^{13} \mathrm{C} \mathrm{NMR}\left(101 \mathrm{MHz}, \mathrm{CDCl}_{3}\right) \delta$ 140.02, 134.91, 128.80, 128.44, 126.04, 68.56, 59.20, 39.61, 23.01, 22.20. HRMS (ESI) Calcd for $\left[\mathrm{C}_{15} \mathrm{H}_{21} \mathrm{BN}, \mathrm{M}-\mathrm{H}\right]^{+}$: 226.1762; Found: 226.1767.

\section{3-benzyl-5 $\lambda^{4}$-aza-1-boraspiro[4.6]undec-2-ene (5g)}<smiles>BrC1=C[B]N2CCCCCC12</smiles>

$65 \%$ yield. Colorless oil. ${ }^{1} \mathrm{H}$ NMR $\left(400 \mathrm{MHz}, \mathrm{CDCl}_{3}\right) \delta 7.38-7.27$ $(\mathrm{m}, 2 \mathrm{H}), 7.22-7.14(\mathrm{~m}, 3 \mathrm{H}), 5.91(\mathrm{~s}, 1 \mathrm{H}), 3.37(\mathrm{~s}, 2 \mathrm{H}), 3.31(\mathrm{~s}, 2 \mathrm{H})$, $3.23(\mathrm{dd}, J=13.7,8.9 \mathrm{~Hz}, 2 \mathrm{H}), 2.87(\mathrm{dd}, J=13.6,8.3 \mathrm{~Hz}, 2 \mathrm{H}), 1.75$ $(\mathrm{dt}, J=15.8,8.0 \mathrm{~Hz}, 2 \mathrm{H}), 1.65-1.51(\mathrm{~m}, 6 \mathrm{H}) .{ }^{13} \mathrm{C} \mathrm{NMR}\left(101 \mathrm{MHz}, \mathrm{CDCl}_{3}\right) \delta 139.92$, 135.43, 128.77, 128.39, 126.01, 69.45, 61.90, 39.66, 28.10, 23.46. HRMS (ESI) Calcd for $\left[\mathrm{C}_{16} \mathrm{H}_{23} \mathrm{BN}, \mathrm{M}-\mathrm{H}\right]^{+}:$240.1918; Found: 240.1916.

\section{3-benzyl-8-oxa-5 $\lambda^{4}$-aza-1-boraspiro[4.5]dec-2-ene (5h)}<smiles></smiles>

$65 \%$ yield. Colorless oil. ${ }^{1} \mathrm{H}$ NMR (400 $\left.\mathrm{MHz}, \mathrm{CDCl}_{3}\right) \delta 7.32-7.26$ (m, 2H), $7.23-7.13(\mathrm{~m}, 3 \mathrm{H}), 5.89(\mathrm{~s}, 1 \mathrm{H}), 4.05$ (ddd, $J=12.4,9.8$, $2.4 \mathrm{~Hz}, 2 \mathrm{H}), 3.70(\mathrm{dt}, J=12.7,3.4 \mathrm{~Hz}, 2 \mathrm{H}), 3.44-3.31(\mathrm{~m}, 4 \mathrm{H})$, $3.17-3.07(\mathrm{~m}, 2 \mathrm{H}), 2.73(\mathrm{ddd}, J=12.7,9.9,3.4 \mathrm{~Hz}, 2 \mathrm{H}) .{ }^{13} \mathrm{C}$ NMR (101 MHz, Chloroform-d) $\delta 139.73,134.52,128.76,128.52,126.20,63.63,58.44,39.48$. HRMS 
(ESI) Calcd for $\left[\mathrm{C}_{14} \mathrm{H}_{19} \mathrm{BNO}, \mathrm{M}-\mathrm{H}\right]^{+}:$228.1554; Found: 228.1555.

\section{1,4-dibenzyl-2,5-dihydro-1H-1,2-azaborole (5i)}

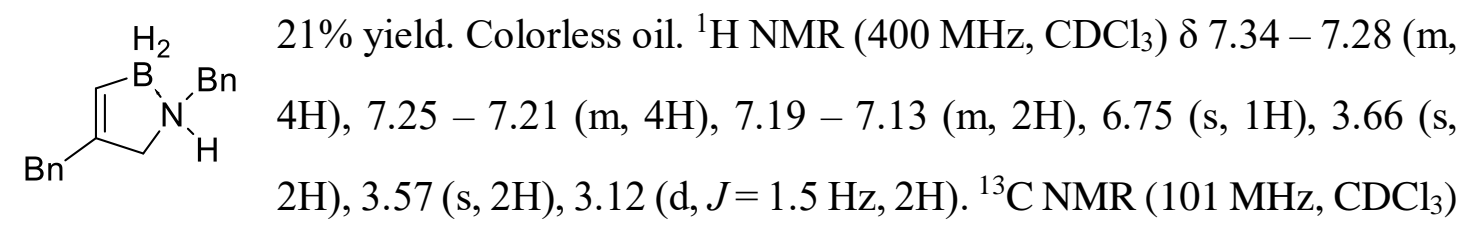
$\delta$ 136.53, 135.28, 133.24, 129.36, 129.30, 129.16, 129.08, 129.01, 128.64, 127.58, 123.60, 60.12, 53.68, 32.78. HRMS (ESI) Calcd for $\left[\mathrm{C}_{17} \mathrm{H}_{21} \mathrm{BN}, \mathrm{M}+\mathrm{H}\right]^{+}$: 250.1762; Found: 250.1773.

\section{(S)-4-benzyl-1-(1-phenylethyl)-2,5-dihydro- $1 H$-1 $\lambda^{4}, 2$-azaborole $(5 \mathrm{j})$}

41\% yield, 95:5 dr. Colorless oil. ${ }^{1} \mathrm{H}$ NMR $\left(400 \mathrm{MHz}, \mathrm{C}_{6} \mathrm{D}_{6}\right) \delta 7.18$
$\mathrm{Hn}$ $1 \mathrm{H}), 3.35(\mathrm{~d}, \mathrm{~J}=14.3 \mathrm{~Hz}, 1 \mathrm{H}), 3.29(\mathrm{q}, \mathrm{J}=6.6 \mathrm{~Hz}, 1 \mathrm{H}), 3.17$ (d, J = $14.3 \mathrm{~Hz}, 1 \mathrm{H}), 2.59$ (dd, J = 14.7, $1.6 \mathrm{~Hz}, 1 \mathrm{H}), 2.51$ (dd, J = 14.6, $1.3 \mathrm{~Hz}, 1 \mathrm{H}), 1.03$ (d, $\mathrm{J}=6.6 \mathrm{~Hz}, 3 \mathrm{H}), 0.74(\mathrm{~b}, 1 \mathrm{H}) .{ }^{13} \mathrm{C} \mathrm{NMR}\left(101 \mathrm{MHz}, \mathrm{C}_{6} \mathrm{D}_{6}\right) \delta 132.89,131.46,129.03$, 128.87, 128.77, 127.38, 126.89, 126.86, 57.67, 46.91, 32.54, 24.46. HRMS (ESI) Calcd for $\left[\mathrm{C}_{18} \mathrm{H}_{26} \mathrm{BN}_{2}, \mathrm{M}+\mathrm{NH}_{4}\right]^{+}: 281.2184$; Found: 281.2178 . The major configuration of 5j is suggested a trans-configuration with $\mathrm{H} 2$ and $\mathrm{H} 6$ based on the 2D NMR spetroscopy and density functional theory calculations calculations.

(7S,8R)-7-((benzo[d][1,3]dioxol-5-yloxy)methyl)-3-benzyl-8-(4-fluorophenyl)-5 $\lambda^{4}$ aza-1-boraspiro[4.5]dec-2-ene (5k)

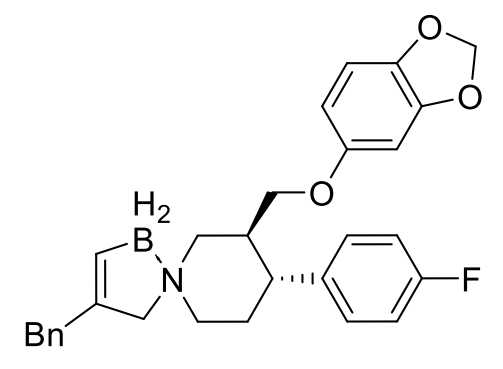

60\% yield, 50:50 dr. Colorless oil. ${ }^{1} \mathrm{H}$ NMR (400 MHz, $\left.\mathrm{CDCl}_{3}\right) \delta 7.28(\mathrm{td}, J=7.7,2.3 \mathrm{~Hz}, 2 \mathrm{H}), 7.18(\mathrm{ddd}, J=$ $11.5,6.8,2.7 \mathrm{~Hz}, 5 \mathrm{H}), 6.96(\mathrm{dt}, J=8.8,4.5 \mathrm{~Hz}, 2 \mathrm{H}), 6.62$ $(\mathrm{dd}, J=8.4,2.5 \mathrm{~Hz}, 1 \mathrm{H}), 6.33(\mathrm{~d}, J=2.6 \mathrm{~Hz}, 1 \mathrm{H}), 6.11$ $(\mathrm{d}, J=8.6 \mathrm{~Hz}, 1 \mathrm{H}), 5.90(\mathrm{~s}, 1 \mathrm{H}), 5.87(\mathrm{~d}, J=2.4 \mathrm{~Hz}, 2 \mathrm{H})$, $3.58-3.45(\mathrm{~m}, 2 \mathrm{H}), 3.42(\mathrm{~s}, 4 \mathrm{H}), 3.39-3.33(\mathrm{~m}, 1 \mathrm{H}), 2.73-2.67(\mathrm{~m}, 2 \mathrm{H}), 2.60-2.47$ $(\mathrm{m}, 2 \mathrm{H}), 1.79-1.69(\mathrm{~m}, 1 \mathrm{H}), 1.33-1.21(\mathrm{~m}, 1 \mathrm{H}), 0.91-0.83(\mathrm{~m}, 1 \mathrm{H}) .{ }^{13} \mathrm{C} \mathrm{NMR}(101$ 
$\left.\mathrm{MHz}, \mathrm{CDCl}_{3}\right) \delta 163.01,160.57,154.21,148.29,141.92,139.78,138.48$ (d, J=3.3 Hz), 135.05, 129.08 (d, $J=7.9 \mathrm{~Hz}), 128.83,128.47,126.12,115.75,115.54,107.98,105.67$, 101.25, 98.06, 73.40, 68.59, 61.98, 59.22, 42.26, 39.52, 39.38, 30.59. HRMS (ESI) Calcd for $\left[\mathrm{C}_{29} \mathrm{H}_{32} \mathrm{BFNO}_{3}, \mathrm{M}+\mathrm{H}\right]^{+}:$472.2459; Found: 472.2451.

\section{4-benzyl-1-(3-(10,11-dihydro-5H-dibenzo[a,d][7]annulen-5-ylidene)propyl)-1-} methyl-2,5-dihydro-1H-1 $\lambda^{4}, 2$-azaborole (5l)<smiles>CN1C[B]C=C(Br)C1</smiles>
$44 \%$ yield. Colorless oil. ${ }^{1} \mathrm{H}$ NMR (400 $\left.\mathrm{MHz}, \mathrm{CDCl}_{3}\right) \delta$ $7.34-7.06(\mathrm{~m}, 12 \mathrm{H}), 7.03(\mathrm{~d}, J=6.9 \mathrm{~Hz}, 1 \mathrm{H}), 5.88(\mathrm{~s}$, $1 \mathrm{H}), 5.71(\mathrm{t}, J=7.5 \mathrm{~Hz}, 1 \mathrm{H}), 3.49-3.19(\mathrm{~m}, 5 \mathrm{H}), 3.05-$ $2.86(\mathrm{~m}, 3 \mathrm{H}), 2.82-2.69(\mathrm{~m}, 2 \mathrm{H}), 2.55-2.44(\mathrm{~m}, 1 \mathrm{H})$, $2.42-2.30(\mathrm{~m}, 4 \mathrm{H}) .{ }^{13} \mathrm{C} \mathrm{NMR}\left(101 \mathrm{MHz}, \mathrm{CDCl}_{3}\right) \delta 145.59,140.73,139.80,139.57$, $139.30,137.16,134.90,130.22,128.79,128.58,128.46,128.34,127.90,127.47$, 126.43, 126.22, 126.10, 126.04, 69.31 (d, $J=48.1 \mathrm{~Hz}), 62.32,48.38(\mathrm{~d}, J=36.3 \mathrm{~Hz})$, 39.54, 33.83, 32.10, 25.28. HRMS (ESI) Calcd for $\left[\mathrm{C}_{29} \mathrm{H}_{32} \mathrm{BNNa}, \mathrm{M}+\mathrm{Na}\right]^{+}$: 428.2526; Found: 428.2519 .

\section{4-benzyl-1-methyl-1-(3-phenyl-3-(4-(trifluoromethyl)phenoxy)propyl)-2,5- dihydro- $1 H-1 \lambda^{4}, 2$-azaborole $(5 \mathrm{~m})$}<smiles></smiles>

$67 \%$ yield, 52:48 dr. Colorless oil. ${ }^{1} \mathrm{H}$ NMR (400 $\left.\mathrm{MHz}, \mathrm{CDCl}_{3}\right) \delta 7.42(\mathrm{~d}, J=8.4 \mathrm{~Hz}, 2 \mathrm{H}), 7.36-7.23$ (m, 7H), $7.21-7.09(\mathrm{~m}, 3 \mathrm{H}), 6.84(\mathrm{dd}, J=8.8,2.4$ $\mathrm{Hz}, 2 \mathrm{H}), 5.91$ (s, 1H), 5.14 (ddd, $J=21.4,8.3,4.4 \mathrm{~Hz}, 1 \mathrm{H}), 3.54-3.27$ (m, 3H), 3.18 $-2.83(\mathrm{~m}, 3 \mathrm{H}), 2.52(\mathrm{~d}, J=8.0 \mathrm{~Hz}, 3 \mathrm{H}), 2.41-2.04(\mathrm{~m}, 2 \mathrm{H}) .{ }^{13} \mathrm{C}$ NMR $(101 \mathrm{MHz}$, $\left.\mathrm{CDCl}_{3}\right) \delta 160.21,140.10,139.90,139.78,139.75,134.91,129.09,128.80,128.50$, 128.38, 128.34, 126.94 (q, $J=3.4 \mathrm{~Hz}), 126.14,125.81,125.73,115.79,78.77$, 78.49, 70.39, 69.54, 60.01, 59.56, 49.15, 48.69, 39.58, 34.30, 33.83. HRMS (ESI) Calcd for $\left[\mathrm{C}_{27} \mathrm{H}_{29} \mathrm{BF}_{3} \mathrm{NNaO}, \mathrm{M}+\mathrm{Na}\right]^{+}:$474.2187; Found: 474.2185. 


\section{1-benzyl-4-(4-chlorobenzyl)-1-methyl-2,5-dihydro- $1 H$-1 $\lambda^{4}, 2$-azaborole (5n)}

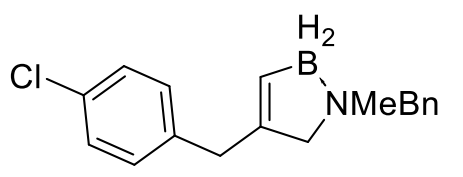

$64 \%$ yield. White solid, mp: $110-111{ }^{\circ} \mathrm{C} .{ }^{1} \mathrm{H}$ NMR (400

$\left.\mathrm{MHz}, \mathrm{CDCl}_{3}\right) \delta 7.39-7.29(\mathrm{~m}, 3 \mathrm{H}), 7.28-7.24(\mathrm{~m}, 2 \mathrm{H})$, $7.13(\mathrm{~d}, J=8.3 \mathrm{~Hz}, 2 \mathrm{H}), 7.10-7.05(\mathrm{~m}, 2 \mathrm{H}), 6.00(\mathrm{~s}, 1 \mathrm{H})$, $3.99(\mathrm{dd}, J=32.1,13.4 \mathrm{~Hz}, 2 \mathrm{H}), 3.58(\mathrm{~d}, J=13.8 \mathrm{~Hz}, 1 \mathrm{H}), 3.41-3.27(\mathrm{~m}, 2 \mathrm{H}), 2.90$ $(\mathrm{d}, J=13.9 \mathrm{~Hz}, 1 \mathrm{H}), 2.46$ (s, 3H). ${ }^{13} \mathrm{C} \mathrm{NMR}\left(101 \mathrm{MHz}, \mathrm{CDCl}_{3}\right) \delta 138.41,134.85$, $132.09,131.93,131.81,130.33,129.04,128.66,128.61,66.40,65.24,47.49,39.04$. HRMS (ESI) Calcd for [ $\left.\mathrm{C}_{18} \mathrm{H}_{21} \mathrm{BCINNa}, \mathrm{M}+\mathrm{Na}\right]^{+}: 320.1348$; Found: 320.1353.

\section{1-benzyl-1-methyl-4-(4-(trifluoromethyl)benzyl)-2,5-dihydro-1H-1 $\lambda^{4}, 2$-azaborole} (5o)<smiles>FC(F)(F)c1ccc(CC2=C[B]N(Cc3ccccc3)C2)cc1</smiles>
$83 \%$ yield. White solid, mp: $105-108{ }^{\circ} \mathrm{C} .{ }^{1} \mathrm{H}$ NMR (400 $\left.\mathrm{MHz} \mathrm{CDCl}_{3}\right) \delta 7.56(\mathrm{~d}, J=8.1 \mathrm{~Hz}, 2 \mathrm{H}), 7.38-7.26(\mathrm{~m}$, $5 \mathrm{H}), 7.07(\mathrm{~d}, J=7.0 \mathrm{~Hz}, 2 \mathrm{H}), 6.03(\mathrm{~s}, 1 \mathrm{H}), 4.03(\mathrm{~d}, J=$ $13.4 \mathrm{~Hz}, 1 \mathrm{H}), 3.95$ (d, $J=13.4 \mathrm{~Hz}, 1 \mathrm{H}), 3.59$ (d, $J=13.9 \mathrm{~Hz}, 1 \mathrm{H}), 3.43$ (q, $J=14.8 \mathrm{~Hz}$, 2H), $2.92(\mathrm{~d}, J=13.9 \mathrm{~Hz}, 1 \mathrm{H}), 2.47(\mathrm{~s}, 3 \mathrm{H}) .{ }^{13} \mathrm{C} \mathrm{NMR}\left(101 \mathrm{MHz}, \mathrm{CDCl}_{3}\right) \delta$ 144.13, 134.12, 132.06, 131.77, 129.29, 129.06, 128.59, 125.48 (q, $J=3.8 \mathrm{~Hz}), 66.50,65.28$, 47.54, 39.46. HRMS (ESI) Calcd for $\left[\mathrm{C}_{19} \mathrm{H}_{21} \mathrm{BF}_{3} \mathrm{NNa}, \mathrm{M}+\mathrm{Na}\right]^{+}$: 354.1611; Found: 354.1617 .

\section{1-benzyl-4-(4-methoxybenzyl)-1-methyl-2,5-dihydro- $1 H$-1 $\lambda^{4}, 2$-azaborole (5p)}

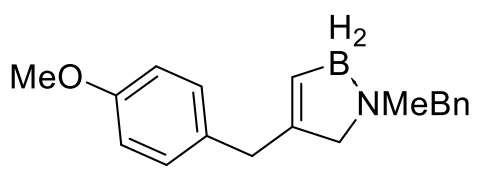

$71 \%$ yield. White solid, mp: $106-108^{\circ} \mathrm{C} .{ }^{1} \mathrm{H}$ NMR (400

$\left.\mathrm{MHz}, \mathrm{CDCl}_{3}\right) \delta 7.37-7.26(\mathrm{~m}, 3 \mathrm{H}), 7.12(\mathrm{~d}, J=8.6 \mathrm{~Hz}$, 2H), $7.09-7.03(\mathrm{~m}, 2 \mathrm{H}), 6.87-6.80(\mathrm{~m}, 2 \mathrm{H}), 5.98(\mathrm{~s}$, 1H), $3.98(\mathrm{dd}, J=39.8,13.4 \mathrm{~Hz}, 2 \mathrm{H}), 3.79$ (s, 3H), 3.58 (d, $J=13.9 \mathrm{~Hz}, 1 \mathrm{H}), 3.33$ (dd, $J=24.1,14.8 \mathrm{~Hz}, 2 \mathrm{H}), 2.92(\mathrm{~d}, J=13.9 \mathrm{~Hz}, 1 \mathrm{H}), 2.45$ (s, 3H). ${ }^{13} \mathrm{C}$ NMR (101 MHz, $\left.\mathrm{CDCl}_{3}\right) \delta 158.08,135.96,132.18,131.97,131.87,129.86,128.91,128.52,113.91$, 66.35, 65.09, 55.40, 47.39, 38.80. HRMS (ESI) Calcd for $\left[\mathrm{C}_{19} \mathrm{H}_{24} \mathrm{BNNaO}, \mathrm{M}+\mathrm{Na}\right]^{+}$: 316.1843; Found: 316.1849. 


\section{1-benzyl-4-(4-fluorobenzyl)-1-methyl-2,5-dihydro- $1 H$-1 $\lambda^{4}, 2$-azaborole $(5 q)$}

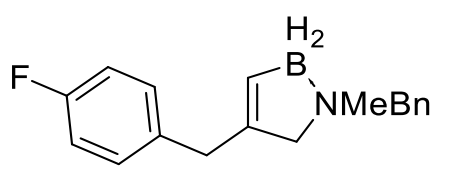

$71 \%$ yield. White solid, mp: $114-116{ }^{\circ} \mathrm{C} .{ }^{1} \mathrm{H}$ NMR (400

$\left.\mathrm{MHz}, \mathrm{CDCl}_{3}\right) \delta 7.41-7.27(\mathrm{~m}, 3 \mathrm{H}), 7.20-7.13(\mathrm{~m}, 2 \mathrm{H})$, $7.10(\mathrm{~d}, J=7.0 \mathrm{~Hz}, 2 \mathrm{H}), 6.98(\mathrm{t}, J=8.6 \mathrm{~Hz}, 2 \mathrm{H}), 5.99(\mathrm{~s}$, $1 \mathrm{H}), 4.00(\mathrm{dd}, J=32.9,13.4 \mathrm{~Hz}, 2 \mathrm{H}), 3.59(\mathrm{~d}, J=13.8 \mathrm{~Hz}, 1 \mathrm{H}), 3.41-3.27(\mathrm{~m}, 2 \mathrm{H})$, $2.91(\mathrm{~d}, J=13.8 \mathrm{~Hz}, 1 \mathrm{H}), 2.46(\mathrm{~s}, 3 \mathrm{H}) .{ }^{13} \mathrm{C} \mathrm{NMR}\left(101 \mathrm{MHz}, \mathrm{CDCl}_{3}\right) \delta 135.39(\mathrm{~d}, J=$ $21.1 \mathrm{~Hz}), 132.18,131.82,130.35,130.28,129.02,128.60,115.27(\mathrm{~d}, J=36.1 \mathrm{~Hz}), 66.58$, 65.29, 47.50, 38.84. HRMS (ESI) Calcd for $\left[\mathrm{C}_{18} \mathrm{H}_{20} \mathrm{BFN}, \mathrm{M}-\mathrm{H}\right]^{+}: 280.1667$; Found: 280.1667.

\section{1-benzyl-1,4-dimethyl-2,5-dihydro- $1 H-1 \lambda^{4}, 2$-azaborole (5r)}<smiles>CC1=C[B]N(Cc2ccccc2)C1</smiles>

$61 \%$ yield. Colorless oil. ${ }^{1} \mathrm{H}$ NMR $\left(400 \mathrm{MHz}, \mathrm{CDCl}_{3}\right) \delta 7.45-7.33$ $(\mathrm{m}, 5 \mathrm{H}), 5.84(\mathrm{~s}, 1 \mathrm{H}), 4.09(\mathrm{dd}, J=41.9,13.4 \mathrm{~Hz}, 2 \mathrm{H}), 3.72(\mathrm{~d}, J=$ $13.9 \mathrm{~Hz}, 1 \mathrm{H}), 2.95(\mathrm{~d}, J=13.9 \mathrm{~Hz}, 1 \mathrm{H}), 2.52(\mathrm{~s}, 3 \mathrm{H}), 1.72(\mathrm{~s}, 3 \mathrm{H})$.

${ }^{13} \mathrm{C} \mathrm{NMR}\left(101 \mathrm{MHz}, \mathrm{CDCl}_{3}\right) \delta 132.50,132.22,131.78,129.04,128.65,69.28,65.72$, 47.79, 18.23. HRMS (ESI) Calcd for $\left[\mathrm{C}_{12} \mathrm{H}_{17} \mathrm{BN}, \mathrm{M}-\mathrm{H}\right]^{+}:$186.1449; Found: 186.1452.

\section{1-benzyl-4-butyl-1-methyl-2,5-dihydro- $1 H-1 \lambda^{4}, 2$-azaborole $(5 \mathrm{~s})$}<smiles>CC(C)(C)C1=C[B]N(Cc2ccccc2)C1</smiles>
$68 \%$ yield. White solid, mp: $42-45{ }^{\circ} \mathrm{C} .{ }^{1} \mathrm{H}$ NMR (400 MHz, $\mathrm{CDCl}_{3}$ ) $\delta 7.44-7.34(\mathrm{~m}, 5 \mathrm{H}), 5.85(\mathrm{~s}, 1 \mathrm{H}), 4.08(\mathrm{dd}, J=38.1,13.4 \mathrm{~Hz}, 2 \mathrm{H})$, $3.73(\mathrm{~d}, J=13.8 \mathrm{~Hz}, 1 \mathrm{H}), 2.99(\mathrm{~d}, J=13.7 \mathrm{~Hz}, 1 \mathrm{H}), 2.51(\mathrm{~s}, 3 \mathrm{H}), 2.10$ $-1.91(\mathrm{~m}, 2 \mathrm{H}), 1.48-1.37(\mathrm{~m}, 2 \mathrm{H}), 1.32(\mathrm{dq}, J=14.2,7.0 \mathrm{~Hz}, 2 \mathrm{H}), 0.90(\mathrm{t}, J=7.2 \mathrm{~Hz}$, $3 \mathrm{H}) .{ }^{13} \mathrm{C} \mathrm{NMR}\left(101 \mathrm{MHz}, \mathrm{CDCl}_{3}\right) \delta 136.98,132.60,131.79,129.03,128.66,68.37$, 65.74, 47.67, 32.09, 30.02, 29.84, 22.74, 14.13. HRMS (ESI) Calcd for $\left[\mathrm{C}_{15} \mathrm{H}_{24} \mathrm{BNNa}\right.$, $\mathrm{M}+\mathrm{Na}]^{+}:$252.1894; Found: 252.1899.

\section{1-benzyl-4-isopropyl-1-methyl-2,5-dihydro- $1 H$ - $1 \lambda^{4}, 2$-azaborole (5t)}

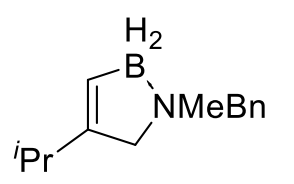

$51 \%$ yield. White solid, mp: $65-69{ }^{\circ} \mathrm{C} .{ }^{1} \mathrm{H}$ NMR (400 MHz, $\mathrm{CDCl}_{3}$ ) $\delta 7.44-7.39(\mathrm{~m}, 3 \mathrm{H}), 7.39-7.35(\mathrm{~m}, 2 \mathrm{H}), 5.85(\mathrm{~s}, 1 \mathrm{H}), 4.14(\mathrm{~d}, J=$ $13.4 \mathrm{~Hz}, 1 \mathrm{H}), 4.06(\mathrm{~d}, J=13.4 \mathrm{~Hz}, 1 \mathrm{H}), 3.77(\mathrm{~d}, J=13.6 \mathrm{~Hz}, 1 \mathrm{H})$, 
$3.06(\mathrm{~d}, J=13.6 \mathrm{~Hz}, 1 \mathrm{H}), 2.50(\mathrm{~s}, 3 \mathrm{H}), 2.30-2.18(\mathrm{~m}, 1 \mathrm{H}), 1.04(\mathrm{~d}, J=6.8 \mathrm{~Hz}, 3 \mathrm{H})$, $1.02(\mathrm{~d}, J=6.8 \mathrm{~Hz}, 3 \mathrm{H}) .{ }^{13} \mathrm{C} \mathrm{NMR}\left(101 \mathrm{MHz}, \mathrm{CDCl}_{3}\right) \delta 142.95,132.59,131.79,129.03$, 128.66, 67.12, 65.70, 47.43, 30.65, 21.44, 21.40. HRMS (ESI) Calcd for $\left[\mathrm{C}_{14} \mathrm{H}_{22} \mathrm{BNNa}\right.$, $\mathrm{M}+\mathrm{Na}]^{+}:$238.1738; Found: 238.1743.

\section{1-benzyl-4-cyclohexyl-1-methyl-2,5-dihydro- $1 H-1 \lambda^{4}, 2$-azaborole (5u)}<smiles>CC(C)CN1BCC(C2CCCCC2)=C1</smiles>

$50 \%$ yield. White solid, m.p.: $116-119{ }^{\circ} \mathrm{C} .{ }^{1} \mathrm{H}$ NMR $(400 \mathrm{MHz}$, $\left.\mathrm{CDCl}_{3}\right) \delta 7.47-7.35(\mathrm{~m}, 5 \mathrm{H}), 5.86(\mathrm{~s}, 1 \mathrm{H}), 4.12(\mathrm{dd}, J=32.1$, $13.4 \mathrm{~Hz}, 2 \mathrm{H}), 3.79$ (d, $J=13.6 \mathrm{~Hz}, 1 \mathrm{H}), 3.07$ (d, $J=13.6 \mathrm{~Hz}, 1 \mathrm{H})$, $2.51(\mathrm{~s}, 3 \mathrm{H}), 1.91(\mathrm{t}, J=11.2 \mathrm{~Hz}, 1 \mathrm{H}), 1.84-1.72(\mathrm{~m}, 4 \mathrm{H}), 1.71-1.64(\mathrm{~m}, 1 \mathrm{H}), 1.34$ -1.09 (m, 5H). ${ }^{13} \mathrm{C}$ NMR (101 MHz, $\left.\mathrm{CDCl}_{3}\right) \delta 142.28,132.55,131.76,128.99,128.63$, 67.12, 65.64, 47.40, 40.69, 31.94, 31.91, 26.58, 26.56, 26.52. HRMS (ESI) Calcd for $\left[\mathrm{C}_{17} \mathrm{H}_{25} \mathrm{BN}, \mathrm{M}-\mathrm{H}\right]^{+}:$254.2075; Found: 254.2073.

\section{1,1-dimethyl-4-phenyl-2,5-dihydro- $1 H$-1 $1 \lambda^{4}, 2$-azaborole (5v)}

$\underset{B}{\mathrm{H}_{2}} \quad 40 \%$ yield. White solid, m.p.: $83-86{ }^{\circ} \mathrm{C} .{ }^{1} \mathrm{H}$ NMR $\left(400 \mathrm{MHz}, \mathrm{CDCl}_{3}\right)$ $\mathrm{NMe}_{2}$

$\delta 7.35-7.27(\mathrm{~m}, 4 \mathrm{H}), 7.21-7.14(\mathrm{~m}, 1 \mathrm{H}), 6.79(\mathrm{~s}, 1 \mathrm{H}), 3.91(\mathrm{~s}, 2 \mathrm{H})$, $2.79(\mathrm{~s}, 6 \mathrm{H}) .{ }^{13} \mathrm{C} \mathrm{NMR}\left(101 \mathrm{MHz}, \mathrm{CDCl}_{3}\right) \delta 136.97,134.83,128.48$, 126.42, 124.56, 70.35, 52.15. HRMS (ESI) Calcd for $\left[\mathrm{C}_{11} \mathrm{H}_{16} \mathrm{BNNa}, \mathrm{M}+\mathrm{Na}\right]^{+}$: 196.1268; Found: 196.1273.

\section{4-(4-methoxyphenyl)-1,1-dimethyl-2,5-dihydro-1H-1 $\lambda^{4}, 2$-azaborole (5w)}<smiles>COc1ccc(C2=C[B]N(C)C2)cc1</smiles>

$28 \%$ yield. White solid, m.p.: $98-101{ }^{\circ} \mathrm{C} .{ }^{1} \mathrm{H}$ NMR $(400 \mathrm{MHz}$, $\left.\mathrm{CDCl}_{3}\right) \delta 7.25(\mathrm{~d}, J=7.9 \mathrm{~Hz}, 2 \mathrm{H}), 6.84(\mathrm{~d}, J=7.9 \mathrm{~Hz}, 2 \mathrm{H})$, $6.61(\mathrm{~s}, 1 \mathrm{H}), 3.88(\mathrm{~s}, 2 \mathrm{H}), 3.79(\mathrm{~s}, 3 \mathrm{H}), 2.79(\mathrm{~s}, 6 \mathrm{H}) .{ }^{13} \mathrm{C} \mathrm{NMR}$ $\left(101 \mathrm{MHz}, \mathrm{CDCl}_{3}\right) \delta 158.31,134.33,130.04,125.70,113.87$, 


\section{4-(4-chlorophenyl)-1,1-dimethyl-2,5-dihydro-1H-1 $\lambda^{4}, 2$-azaborole $(5 x)$}<smiles>CN1[B]CC(c2ccc(Cl)cc2)=C1</smiles>

$42 \%$ yield. White solid, m.p.: $99-102{ }^{\circ} \mathrm{C} .{ }^{1} \mathrm{H}$ NMR (400 MHz, $\left.\mathrm{CDCl}_{3}\right) \delta 7.31-7.18(\mathrm{~m}, 4 \mathrm{H}), 6.79(\mathrm{~s}, 1 \mathrm{H}), 3.89(\mathrm{~s}, 2 \mathrm{H}), 2.80$ (s, 6H). ${ }^{13} \mathrm{C}$ NMR (101 MHz, $\left.\mathrm{CDCl}_{3}\right) \delta 135.42,133.67,131.98$, 128.58, 125.81, 70.24, 52.22. HRMS (ESI) Calcd for

$\left[\mathrm{C}_{11} \mathrm{H}_{15} \mathrm{BClNNa}, \mathrm{M}+\mathrm{Na}\right]^{+}: 230.0878$; Found: 230.0883 .

\section{4-(4-trifluoromethy)-1,1-dimethyl-2,5-dihydro- $1 H-1 \lambda^{4}, 2$-azaborole (5y)}<smiles>CB1C=C(c2ccc(C(F)(F)F)cc2)CN1</smiles>

$52 \%$ yield. Colorless oil. ${ }^{1} \mathrm{H}$ NMR $\left(400 \mathrm{MHz}, \mathrm{CDCl}_{3}\right) \delta 7.71$ $(\mathrm{d}, J=8.1 \mathrm{~Hz}, 2 \mathrm{H}), 7.49(\mathrm{~d}, J=8.1 \mathrm{~Hz}, 2 \mathrm{H}), 7.08(\mathrm{~s}, 1 \mathrm{H}), 4.09$ (s, 2H), 2.47 (s, 6H). $\left.{ }^{13} \mathrm{C} \mathrm{NMR} \mathrm{(101} \mathrm{MHz,} \mathrm{CDCl}_{3}\right) \delta$ 139.57, $139.26,131.68(\mathrm{q}, J=33.3 \mathrm{~Hz}), 127.46,126.63(\mathrm{q}, J=3.1 \mathrm{~Hz})$,

124.65, 123.60, 122.38, 120.14, 116.95, 58.79, 51.66. HRMS (ESI) Calcd for $\left[\mathrm{C}_{12} \mathrm{H}_{15} \mathrm{BF}_{3} \mathrm{NNa}, \mathrm{M}+\mathrm{Na}\right]^{+}:$264.1147; Found: 264.1152. 


\section{X-ray Diffraction Analysis of Product 5q}

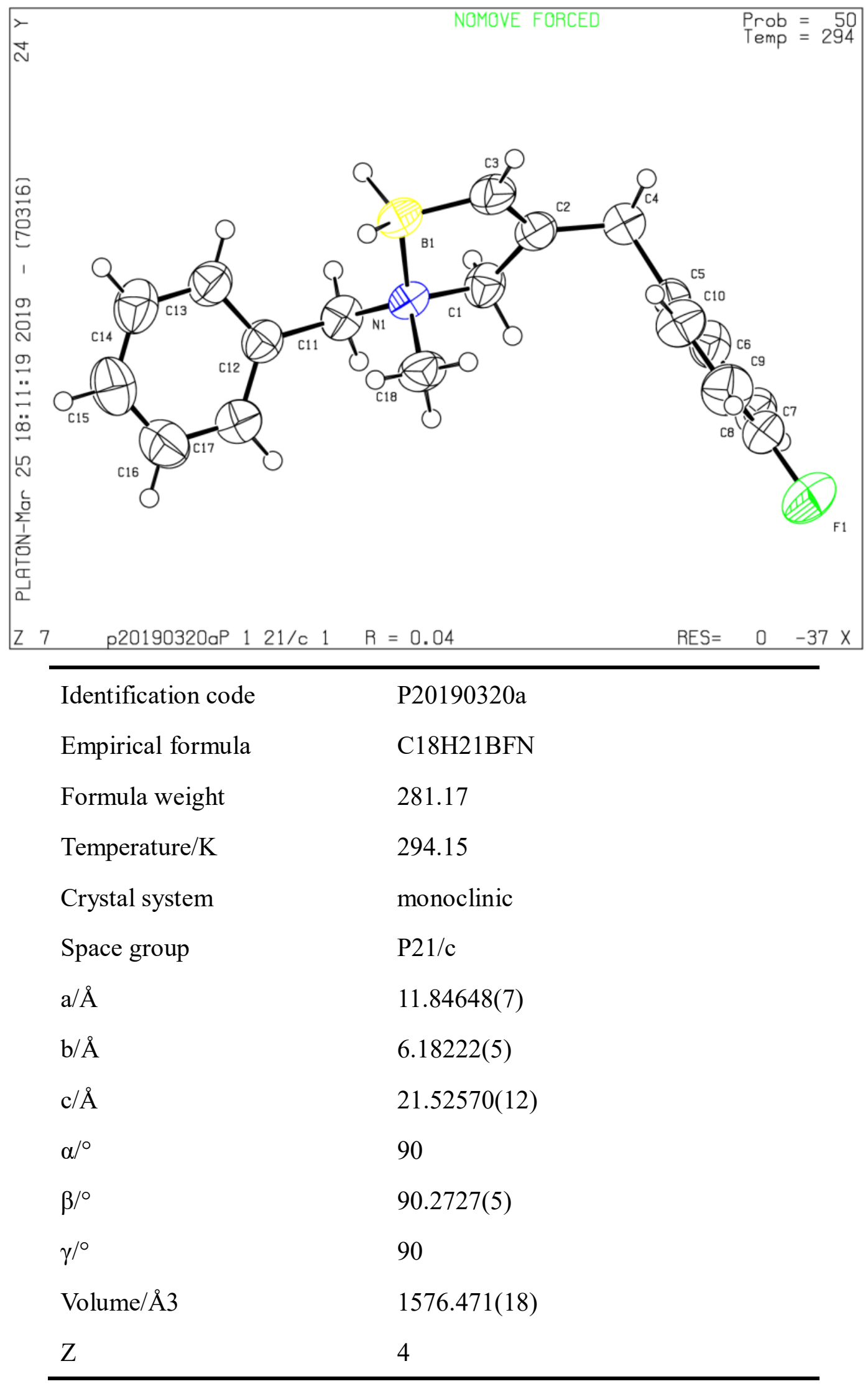




\begin{tabular}{ll}
\hline$\rho c a l c g / \mathrm{cm} 3$ & 1.185 \\
$\mu / \mathrm{mm}-1$ & 0.595 \\
$\mathrm{~F}(000)$ & 600.0 \\
Crystal size/mm3 & $0.36 \times 0.24 \times 0.22$ \\
Radiation & $\mathrm{CuK} \alpha(\lambda=1.54184)$ \\
$2 \Theta$ range for data collection/ ${ }^{\circ}$ & 7.462 to 158.722 \\
Index ranges & $-15 \leq \mathrm{h} \leq 14,-6 \leq \mathrm{k} \leq 7,-27 \leq 1 \leq 27$ \\
Reflections collected & 19103 \\
Independent reflections & $3323[\mathrm{Rint}=0.0290, \mathrm{Rsigma}=0.0181]$ \\
Data/restraints/parameters & $3323 / 0 / 200$ \\
Goodness-of-fit on F2 & 1.063 \\
Final R indexes [I $>=2 \sigma(\mathrm{I})]$ & $\mathrm{R} 1=0.0413, \mathrm{wR} 2=0.1148$ \\
Final R indexes [all data] & $\mathrm{R} 1=0.0434, \mathrm{wR} 2=0.1167$ \\
Largest diff. peak/hole $/ \mathrm{e} \AA-3$ & $0.18 /-0.13$ \\
\hline
\end{tabular}




\section{Transformations of the Products}

\subsection{Synthesis of (4-benzylidenecyclohexyl)benzene (6)}

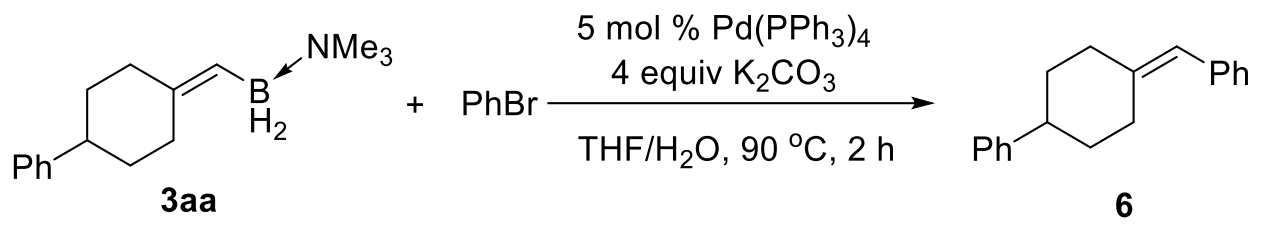

Under an argon atmosphere, 3aa (48.6 mg, $0.2 \mathrm{mmol}), \mathrm{K}_{2} \mathrm{CO}_{3}(110.5 \mathrm{mg}, 0.8 \mathrm{mmol})$ and $\mathrm{Pd}\left(\mathrm{PPh}_{3}\right)_{4}(11.5 \mathrm{mg}, 0.01 \mathrm{mmol})$ were introduced into a $25 \mathrm{~mL}$ Schlenk tube. Then THF (2 mL), PhBr (37.7 mg, $0.24 \mathrm{mmol})$ and water $(0.5 \mathrm{~mL})$ were sequentially injected into the Schlenk tube. The resulting mixture was stirred at $90{ }^{\circ} \mathrm{C}$ for $2 \mathrm{~h}$. After cooling to room temperature, the reaction was diluted by water, extracted with ether. The organic layer was concentrated under vacuum. The product was purified by silica gel column (PE) to afford $\mathbf{6}(41.2 \mathrm{mg}, 0.166 \mathrm{mmol}, 83 \%)$ as a colorless oil. Compound 6 was confirmed by comparing the spectra data with this reported in the literature. ${ }^{6}{ }^{1} \mathrm{H}$ NMR $\left(400 \mathrm{MHz}, \mathrm{CDCl}_{3}\right) \delta 7.34-7.15(\mathrm{~m}, 10 \mathrm{H}), 6.31(\mathrm{~s}, 1 \mathrm{H}), 3.03(\mathrm{ddt}, \mathrm{J}=13.6,4.2,2.4$ $\mathrm{Hz}, 1 \mathrm{H}), 2.75(\mathrm{tt}, \mathrm{J}=12.1,3.5 \mathrm{~Hz}, 1 \mathrm{H}), 2.54-2.33(\mathrm{~m}, 2 \mathrm{H}), 2.11-2.00(\mathrm{~m}, 2 \mathrm{H}), 2.00$ $-1.92(\mathrm{~m}, 1 \mathrm{H}), 1.65(\mathrm{qd}, \mathrm{J}=12.7,4.3 \mathrm{~Hz}, 1 \mathrm{H}), 1.53(\mathrm{qd}, \mathrm{J}=12.8,3.9 \mathrm{~Hz}, 1 \mathrm{H}) .{ }^{13} \mathrm{C}$ NMR $\left(101 \mathrm{MHz} \mathrm{CDCl}_{3}\right) \delta 141.97,138.29,129.05,128.47,128.18,126.94,126.13$, $126.07,122.88,44.60,37.49,35.88,35.13,29.27$.

\subsection{Synthesis of (4-(3-methylbut-2-en-1-ylidene)cyclohexyl)benzene (7)}<smiles>CNBC=C1CCC(c2ccccc2)CC1</smiles>

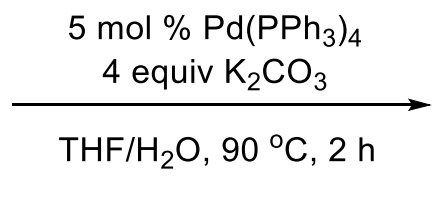<smiles>CC(C)=CC=C1CCC(c2ccccc2)CC1</smiles>

Under an argon atmosphere, 3aa (48.6 mg, $0.2 \mathrm{mmol}), \mathrm{K}_{2} \mathrm{CO}_{3}(110.5 \mathrm{mg}, 0.8 \mathrm{mmol})$ and $\mathrm{Pd}\left(\mathrm{PPh}_{3}\right)_{4}(11.5 \mathrm{mg}, 0.01 \mathrm{mmol})$ were introduced into a $25 \mathrm{~mL}$ Schlenk tube. Then THF (2 mL), 1-bromo-2-methylprop-1-ene $(54.0 \mathrm{mg}, 0.4 \mathrm{mmol})$ and water $(0.5 \mathrm{~mL})$ were sequentially injected into the Schlenk tube. The resulting mixture was stirred at $90{ }^{\circ} \mathrm{C}$ for $2 \mathrm{~h}$. After cooling to room temperature, the reaction was diluted by water, 
extracted with ether. The organic layer was concentrated under vacuum. The product was purified by silica gel column (PE) to afford compound 7 (36.2 mg, $0.16 \mathrm{mmol}$, 90\%) as a colorless oil. ${ }^{1} \mathrm{H}$ NMR $\left(400 \mathrm{MHz}, \mathrm{CDCl}_{3}\right) \delta 7.32-7.25(\mathrm{~m}, 2 \mathrm{H}), 7.25-7.13$ (m, 3H), 6.07 (d, $J=11.5 \mathrm{~Hz}, 1 \mathrm{H}), 6.01$ (d, $J=11.4 \mathrm{~Hz}, 1 \mathrm{H}), 2.98-2.87$ (m, 1H), 2.70 (tt, $J=12.3,3.4 \mathrm{~Hz}, 1 \mathrm{H}), 2.43-2.33(\mathrm{~m}, 1 \mathrm{H}), 2.33-2.22(\mathrm{~m}, 1 \mathrm{H}), 2.04-1.89(\mathrm{~m}, 3 \mathrm{H})$, $1.81(\mathrm{~s}, 3 \mathrm{H}), 1.77(\mathrm{~s}, 3 \mathrm{H}), 1.64-1.53(\mathrm{~m}, 1 \mathrm{H}), 1.53-1.42(\mathrm{~m}, 1 \mathrm{H}) .{ }^{13} \mathrm{C}$ NMR $(101$ $\left.\mathrm{MHz}, \mathrm{CDCl}_{3}\right) \delta 147.05,139.11,133.40,128.47,126.98,126.10,120.51,118.89,44.95$, 37.46, 36.04, 35.11, 28.81, 26.52, 18.22. HRMS (ESI) Calcd for $\left[\mathrm{C}_{17} \mathrm{H}_{21}, \mathrm{M}-\mathrm{H}\right]^{+}$: 225.1638; Found: 225.1638.

\subsection{Synthesis of 1-((4-phenylcyclohexylidene)methyl)-1H-imidazole (8)}

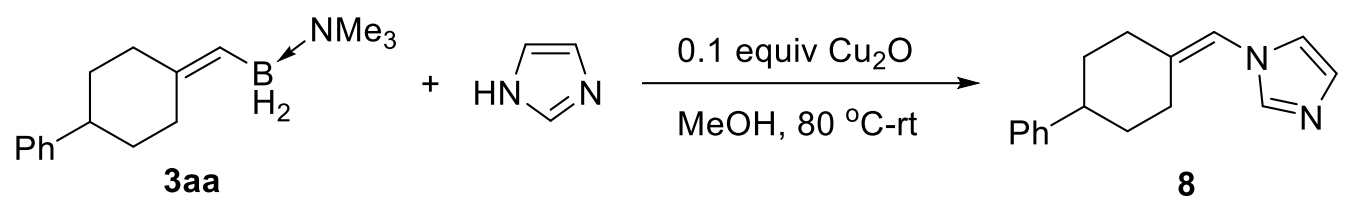

Into a dry Schlenk tube, was added 3aa $(48.6 \mathrm{mg}, 0.2 \mathrm{mmol})$ and dry $\mathrm{MeOH}(2 \mathrm{~mL})$, then stirred at $80{ }^{\circ} \mathrm{C}$ for $1 \mathrm{~h}$. After cooling to room temperature, $\mathrm{Cu}_{2} \mathrm{O}(2.8 \mathrm{mg}, 0.02$ mmol) and $1 H$-imidazole $(16.3 \mathrm{mg}, 0.24 \mathrm{mmol})$ were introduced to the reaction mixture. The resulting mixture was stirred under an air atmosphere at room temperature for $5 \mathrm{~h}$. The product was purified by flash column chromatography on silica gel (PE/EA $=1: 1$, $v / v)$ to afford 8 (32.8 $\mathrm{mg}, 0.138 \mathrm{mmol}, 69 \%)$ as a white solid, mp: $83-85{ }^{\circ} \mathrm{C} .{ }^{1} \mathrm{H}$ NMR $\left(400 \mathrm{MHz} \mathrm{CDCl}_{3}\right) \delta 7.40(\mathrm{~s}, 1 \mathrm{H}), 7.27-7.19(\mathrm{~m}, 2 \mathrm{H}), 7.16-7.09(\mathrm{~m}, 3 \mathrm{H}), 7.02(\mathrm{~s}, 1 \mathrm{H})$, $6.84(\mathrm{~s}, 1 \mathrm{H}), 6.42(\mathrm{~s}, 1 \mathrm{H}), 2.68(\mathrm{tt}, J=12.1,3.3 \mathrm{~Hz}, 1 \mathrm{H}), 2.57$ (dt, $J=14.7,3.6 \mathrm{~Hz}, 1 \mathrm{H})$, 2.47-2.38 (m, 1H), 2.30-2.16 (m, 1H), 2.04-1.86(m, 3H), $1.54(\mathrm{td}, J=12.7,4.1 \mathrm{~Hz}$, 1H), 1.49-1.34 (m, 1H). ${ }^{13} \mathrm{C} \mathrm{NMR}\left(101 \mathrm{MHz}, \mathrm{CDCl}_{3}\right) \delta 145.97,140.20,137.55,129.11$, 128.56, 126.82, 126.38, 119.88, 116.22, 44.13, 35.14, 34.52, 33.26, 27.95. HRMS (ESI) Calcd for $\left[\mathrm{C}_{16} \mathrm{H}_{19} \mathrm{~N}_{2}, \mathrm{M}+\mathrm{H}\right]^{+}:$239.1543; Found: 239.1545 . 
6.4. Synthesis of (4-(chloromethylene)cyclohexyl)benzene (9a) and (4(bromomethylene)cyclohexyl)benzene (9b)

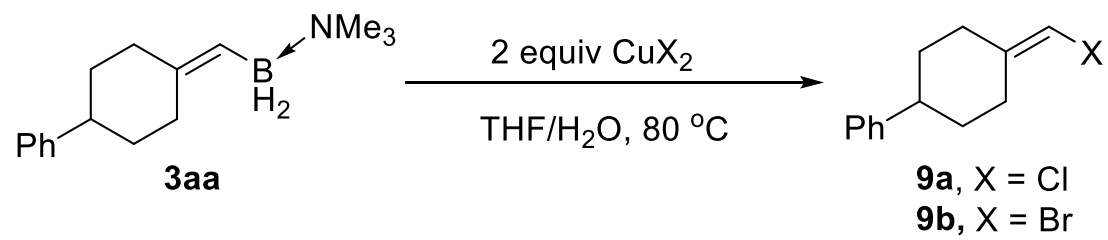

Under an argon atmosphere, 3aa $(48.6 \mathrm{mg}, 0.2 \mathrm{mmol})$ was introduced into a $25 \mathrm{~mL}$ Schlenk tube. Then THF $(1.5 \mathrm{~mL})$ and water $(0.5 \mathrm{~mL})$ were injected into the Schlenk tube. After stirred at $80^{\circ} \mathrm{C}$ for $1 \mathrm{~h}, \mathrm{CuCl}_{2}$ or $\mathrm{CuBr}_{2}(0.4 \mathrm{mmol}, 2$ equiv) were introduced to the resulting mixture. After $8 \mathrm{~h}$ at $80^{\circ} \mathrm{C}$, the resulting mixture was extracted with EtOAc $(5 \mathrm{~mL})$ and the organic layer was dried over $\mathrm{MgSO}_{4}$. After filtration and evaporation of all volatiles, the residue was purified by column chromatography (PE). 9a (30.0 mg, $0.145 \mathrm{mmol}, 73 \%)$. Colorless oil. ${ }^{1} \mathrm{H}$ NMR (400 MHz, $\left.\mathrm{CDCl}_{3}\right) \delta 7.33-$ $7.25(\mathrm{~m}, 2 \mathrm{H}), 7.24-7.15(\mathrm{~m}, 3 \mathrm{H}), 5.84(\mathrm{t}, J=2.1 \mathrm{~Hz}, 1 \mathrm{H}), 3.10-3.01(\mathrm{~m}, 1 \mathrm{H}), 2.69$ $(\mathrm{tt}, J=12.2,3.5 \mathrm{~Hz}, 1 \mathrm{H}), 2.47-2.37(\mathrm{~m}, 1 \mathrm{H}), 2.25-2.12(\mathrm{~m}, 1 \mathrm{H}), 2.07-1.86(\mathrm{~m}, 3 \mathrm{H})$, $1.61-1.40(\mathrm{~m}, 2 \mathrm{H}) .{ }^{13} \mathrm{C} \mathrm{NMR}\left(101 \mathrm{MHz}, \mathrm{CDCl}_{3}\right) \delta 146.39,140.97,128.55,126.92$, 126.30, 109.31, 44.33, 35.18, 34.00, 33.95, 28.46. HRMS (ESI) Calcd for $\left[\mathrm{C}_{13} \mathrm{H}_{15} \mathrm{CINa}\right.$, $\mathrm{M}+\mathrm{Na}]^{+}:$229.0760; Found: 229.0766.

9b (40.9 mg, $0.163 \mathrm{mmol}, 82 \%$ ). 9b was confirmed by comparing the spectra data with those reported in the literature. ${ }^{7}$ Colorless oil. ${ }^{1} \mathrm{H}$ NMR $\left(400 \mathrm{MHz}, \mathrm{CDCl}_{3}\right) \delta$ $7.35-7.29(\mathrm{~m}, 2 \mathrm{H}), 7.25-7.19(\mathrm{~m}, 3 \mathrm{H}), 5.95(\mathrm{t}, J=1.9 \mathrm{~Hz}, 1 \mathrm{H}), 3.11-3.01(\mathrm{~m}$, 1H), 2.73 (tt, $J=12.3,3.4 \mathrm{~Hz}, 1 \mathrm{H}), 2.52(\mathrm{ddt}, J=13.6,4.1,2.3 \mathrm{~Hz}, 1 \mathrm{H}), 2.29-2.17$ (m, 1H), $2.08-1.93(\mathrm{~m}, 3 \mathrm{H}), 1.62-1.50(\mathrm{~m}, 2 \mathrm{H}) .{ }^{13} \mathrm{C} \mathrm{NMR}\left(101 \mathrm{MHz}, \mathrm{CDCl}_{3}\right) \delta$ $146.25,143.99,128.56,126.93,126.32,98.50,44.23,35.40,35.16,33.97,31.06$. 


\subsection{Synthesis of 4,4,5,5-tetramethyl-2-((4-phenylcyclohexylidene)methyl)-1,3,2-}

dioxaborolane (10)<smiles>CN(C)C=C1CCC(c2ccccc2)CC1</smiles>

3aa

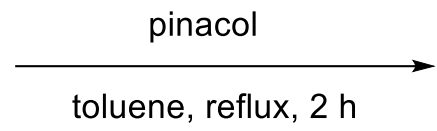

toluene, reflux, $2 \mathrm{~h}$<smiles>BrC(Br)=C1CCC(c2ccccc2)CC1</smiles>

To a solution of 3aa (48.6 mg, $0.2 \mathrm{mmol})$ in toluene $(2 \mathrm{~mL})$, pinacol (anhydrous, 236 $\mathrm{mg}, 2 \mathrm{mmol}$ ) was added under nitrogen atmosphere. The resulting mixture was heated to reflux for $2 \mathrm{~h}$. After concentration in vacuo, the residue was purified by column chromatography $(\mathrm{PE} / \mathrm{EA}=2: 1, v / v)$ to give $10(44.7 \mathrm{mg}, 0.15 \mathrm{mmol}, 75 \%)$ as a white solid, mp: $77-80{ }^{\circ} \mathrm{C} .{ }^{1} \mathrm{H}$ NMR $\left(400 \mathrm{MHz}, \mathrm{CDCl}_{3}\right) \delta 7.30-7.24(\mathrm{~m}, 2 \mathrm{H}), 7.23-7.13$ (m, 3H), $5.11(\mathrm{~s}, 1 \mathrm{H}), 3.31(\mathrm{~d}, J=13.7 \mathrm{~Hz}, 1 \mathrm{H}), 2.72(\mathrm{t}, J=12.0 \mathrm{~Hz}, 1 \mathrm{H}), 2.40-2.29$ (m Hz, 2H), $2.10-1.95(\mathrm{~m}, 3 \mathrm{H}), 1.65-1.55(\mathrm{~m}, 2 \mathrm{H}), 1.27(\mathrm{~s}, 12 \mathrm{H}) .{ }^{13} \mathrm{C}$ NMR $(101$ $\left.\mathrm{MHz}, \mathrm{CDCl}_{3}\right) \delta 165.46,146.89,128.46,126.99,126.10,82.79,44.39,39.94,36.02$, 35.62, 32.97, 25.04, 24.93. HRMS (ESI) Calcd for $\left[\mathrm{C}_{19} \mathrm{H}_{27} \mathrm{BNaO}_{2}, \mathrm{M}+\mathrm{Na}\right]^{+}: 321.1996$; Found: 321.2000 .

\subsection{Synthesis of 4-methyl-8-((4-phenylcyclohexylidene)methyl)dihydro- $4 \lambda^{4}, 8 \lambda^{4}-$}

\section{$[1,3,2]$ oxazaborolo $[2,3-b][1,3,2]$ oxazaborole-2,6(3H,5H)-dione (11)}

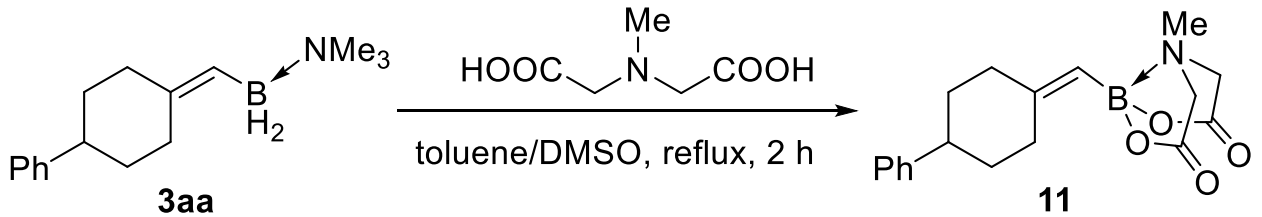

$25 \mathrm{~mL}$ Schlenk tube was charged with $3 \mathbf{a a}(48.6 \mathrm{mg}, 0.2 \mathrm{mmol}), N$-methyliminodiacetic acid (58.8 mg, $0.4 \mathrm{mmol}$ ). Then $2 \mathrm{~mL}$ solvent (toluene/DMSO = 5:1) was injected under nitrogen atmosphere. The resulting mixture was heated to reflux for $2 \mathrm{~h}$. After concentration in vacuo, the residue was purified by column chromatography $\left(\mathrm{Et}_{2} \mathrm{O} /\right.$ acetone $\left.=2: 1, v / v\right)$ to give $11(53.7 \mathrm{mg}, 0.164 \mathrm{mmol}, 82 \%)$ as a white solid, $\mathrm{mp}:$ 180-184 ${ }^{\circ} \mathrm{C} .{ }^{1} \mathrm{H}$ NMR $\left(400 \mathrm{MHz}\right.$, Acetonitrile- $\left.d_{3}\right) \delta 7.31-7.21(\mathrm{~m}, 4 \mathrm{H}), 7.17(\mathrm{tt}, J=$ $6.2,1.6 \mathrm{~Hz}, 1 \mathrm{H}), 5.01(\mathrm{~s}, 1 \mathrm{H}), 3.94(\mathrm{~d}, J=17.0 \mathrm{~Hz}, 2 \mathrm{H}), 3.78(\mathrm{dd}, J=17.0,2.1 \mathrm{~Hz}, 2 \mathrm{H})$, $2.95-2.85(\mathrm{~m}, 1 \mathrm{H}), 2.82(\mathrm{~s}, 3 \mathrm{H}), 2.75(\mathrm{tt}, J=12.1,3.4 \mathrm{~Hz}, 1 \mathrm{H}), 2.40-2.35(\mathrm{~m}, 1 \mathrm{H})$, $2.07-1.98(\mathrm{~m}, 1 \mathrm{H}), 1.96(\mathrm{~s}, 3 \mathrm{H}), 1.65-1.40(\mathrm{~m}, 2 \mathrm{H}) .{ }^{13} \mathrm{C}$ NMR $(101 \mathrm{MHz}$, 
Acetonitrile- $\left.d_{3}\right) \delta 169.24,169.19,157.37,147.73,129.07,127.55,126.69,62.11,47.29$, 44.74, 40.85, 36.67, 36.11, 31.96. HRMS (ESI) Calcd for $\left[\mathrm{C}_{18} \mathrm{H}_{22} \mathrm{BNNaO}_{4}, \mathrm{M}+\mathrm{Na}\right]^{+}$: 350.1534; Found: 350.1538.

\subsection{Synthesis of $(Z)-3-($ benzo $[d][1,3]$ dioxol-5-yl)- $N$,2-dibenzyl- $N$-methylprop-2- en-1-amine (12)}<smiles></smiles>

$5 c$

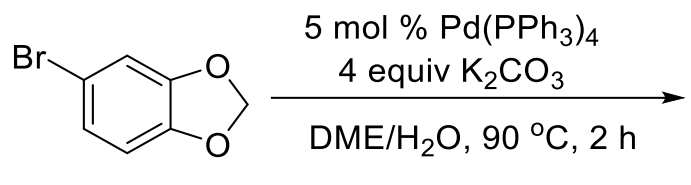<smiles>CN(Br)C/C(=C\c1ccc2c(c1)OCO2)Cc1ccccc1</smiles>

12

Under an argon atmosphere, $\mathbf{5 c}(63.2 \mathrm{mg}, 0.24 \mathrm{mmol}), \mathrm{K}_{2} \mathrm{CO}_{3}(110.5 \mathrm{mg}, 0.8 \mathrm{mmol})$ and $\mathrm{Pd}\left(\mathrm{PPh}_{3}\right)_{4}(11.5 \mathrm{mg}, 0.01 \mathrm{mmol})$ were introduced into a $25 \mathrm{~mL}$ Schlenk tube. Then DME (2 mL), 5-bromobenzo[d][1,3]dioxole (40.2 $\mathrm{mg}, 0.2 \mathrm{mmol})$ and water $(0.5 \mathrm{~mL})$ were sequentially injected into the Schlenk tube. The resulting mixture was stirred at $90{ }^{\circ} \mathrm{C}$ for $2 \mathrm{~h}$. After cooling to room temperature, the reaction was diluted by water, extracted with ether. The organic layer was concentrated under vacuum. The product was purified by silica gel column $(\mathrm{PE} / \mathrm{EA}=10: 1, v / v)$ to afford $12(60.2 \mathrm{mg}, 0.162$ mmol, 81\%) as a colorless oil. ${ }^{1} \mathrm{H}$ NMR $\left(400 \mathrm{MHz}, \mathrm{CDCl}_{3}\right) \delta 7.33-7.17(\mathrm{~m}, 10 \mathrm{H})$, $6.78-6.71(\mathrm{~m}, 2 \mathrm{H}), 6.68-6.60(\mathrm{~m}, 1 \mathrm{H}), 6.42(\mathrm{~s}, 1 \mathrm{H}), 5.92(\mathrm{~s}, 2 \mathrm{H}), 3.63(\mathrm{~d}, J=1.3 \mathrm{~Hz}$, 2H), $3.38(\mathrm{~s}, 2 \mathrm{H}), 3.05(\mathrm{~d}, J=1.1 \mathrm{~Hz}, 2 \mathrm{H}), 2.06(\mathrm{~s}, 3 \mathrm{H}) .{ }^{13} \mathrm{C} \mathrm{NMR}\left(101 \mathrm{MHz}, \mathrm{CDCl}_{3}\right)$ $\delta 147.37,146.10,140.42,140.31,139.65,131.79,129.51,129.38,128.99,128.35$, $128.29,126.93,126.07,122.81,109.67,108.02,101.00,61.89,56.00,42.36,42.09$. HRMS (ESI) Calcd for [ $\left.\mathrm{C}_{25} \mathrm{H}_{26} \mathrm{NO}_{2}, \mathrm{M}+\mathrm{H}\right]^{+}: 372.1958$; Found: 372.1962. 


\subsection{Synthesis of $(Z)-N, 2$-dibenzyl-3-(1H-indol-5-yl)- $N$-methylprop-2-en-1-amine}

(13)<smiles>COC(=O)c1cc2cc(Br)ccc2[nH]1</smiles><smiles>CN(C/C(=C\c1ccc2[nH]ccc2c1)Cc1ccccc1)Cc1ccccc1</smiles>

13

Under an argon atmosphere, 5c (63.2 mg, $0.24 \mathrm{mmol}), \mathrm{K}_{2} \mathrm{CO}_{3}(110.5 \mathrm{mg}, 0.8 \mathrm{mmol})$ and $\mathrm{Pd}\left(\mathrm{PPh}_{3}\right)_{4}(11.5 \mathrm{mg}, 0.01 \mathrm{mmol})$ were introduced into a $25 \mathrm{~mL}$ Schlenk tube. Then DME $(2 \mathrm{~mL}), 5$-bromo-1H-indole $(39.2 \mathrm{mg}, 0.2 \mathrm{mmol})$ and water $(0.5 \mathrm{~mL})$ were sequentially injected into the Schlenk tube. The resulting mixture was stirred at $90{ }^{\circ} \mathrm{C}$ for $2 \mathrm{~h}$. After cooling to room temperature, the reaction was diluted by water, extracted with ether. The organic layer was concentrated under vacuum. The product was purified by silica gel column $(\mathrm{PE} / \mathrm{EA}=5: 1, v / v)$ to afford $13(52.8 \mathrm{mg}, 0.144 \mathrm{mmol}, 72 \%)$ as a colorless oil. ${ }^{1} \mathrm{H}$ NMR (400 MHz, $\left.\mathrm{CDCl}_{3}\right) \delta 8.06(\mathrm{~s}, 1 \mathrm{H}), 7.46(\mathrm{~s}, 1 \mathrm{H}), 7.36-7.13(\mathrm{~m}$, 12H), $7.04(\mathrm{dd}, J=8.4,1.6 \mathrm{~Hz}, 1 \mathrm{H}), 6.68(\mathrm{~s}, 1 \mathrm{H}), 6.51(\mathrm{~s}, 1 \mathrm{H}), 3.69(\mathrm{~s}, 2 \mathrm{H}), 3.36$ (s, 2H), $3.14(\mathrm{~s}, 2 \mathrm{H}), 2.05(\mathrm{~s}, 3 \mathrm{H}) .{ }^{13} \mathrm{C} \mathrm{NMR}\left(101 \mathrm{MHz}, \mathrm{CDCl}_{3}\right) \delta 140.78,139.84,139.03$, $134.52,130.82,129.55,129.32,128.86,128.19,128.13,127.69,126.71,125.83$, 124.41, 123.88, 121.17, 110.41, 102.74, 61.71, 55.93, 42.21, 41.98. HRMS (ESI) Calcd for $\left[\mathrm{C}_{26} \mathrm{H}_{27} \mathrm{~N}_{2}, \mathrm{M}+\mathrm{H}\right]^{+}: 367.2169$; Found: 367.2172 . 


\section{Kinetic Isotope Effect Experiments}

\subsection{Labeling experiment using $\mathrm{BH}_{3}$ and $\mathrm{BD}_{3}$ adducts}

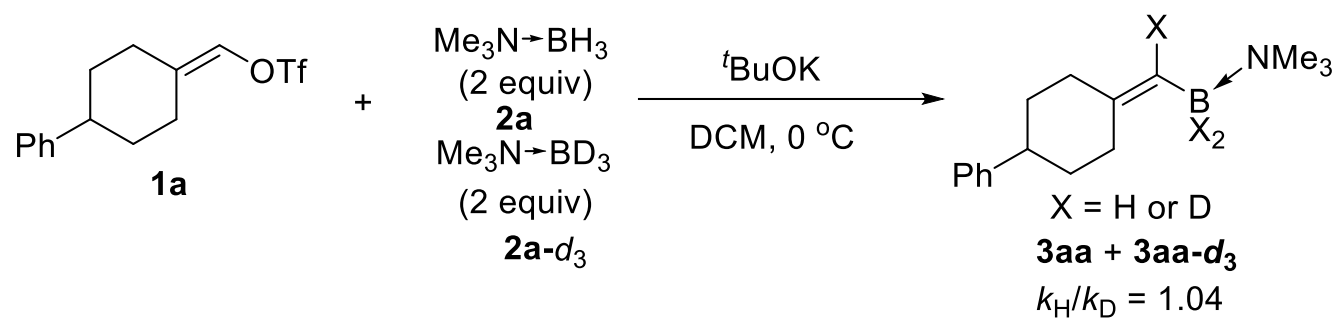

Under an argon atmosphere, 2a (43.8 mg, $0.6 \mathrm{mmol}), \mathbf{2 a}-d_{3}(45.5 \mathrm{mg}, 0.6 \mathrm{mmol})$ and ${ }^{t} \mathrm{BuOK}(40.4 \mathrm{mg}, 0.36 \mathrm{mmol})$ were introduced into a Schlenk tube. Then $\mathrm{CH}_{2} \mathrm{Cl}_{2}(3 \mathrm{~mL})$ was injected into the Schlenk tube in one portion at $0{ }^{\circ} \mathrm{C}$. Alkenyl triflate 1a $(96.1 \mathrm{mg}$, $0.3 \mathrm{mmol})$ was then added into this reaction mixture in one portion. The resulting mixture was stirred at $0{ }^{\circ} \mathrm{C}$ for $2 \mathrm{~h}$. The product was purified by flash column chromatography on silica gel $\left(\mathrm{PE} / \mathrm{EA} / \mathrm{Et}_{3} \mathrm{~N}=15: 1: 0.05, v / v / v\right)$ to afford $\mathrm{B}-\mathrm{H}$ insertion product $(55.7 \mathrm{mg}, 76 \%)$. The deuterium distribution of the product was determined by ${ }^{1} \mathrm{H}$ NMR of the crude product. ${ }^{1} \mathrm{H} \mathrm{NMR}\left(400 \mathrm{MHz}, \mathrm{CDCl}_{3}\right) \delta 7.30-7.24(\mathrm{~m}, 2 \mathrm{H}), 7.24$ $-7.19(\mathrm{~m}, 2 \mathrm{H}), 7.19-7.13(\mathrm{~m}, 1 \mathrm{H}), 5.48-5.33(\mathrm{~m}, 0.51 \mathrm{H}), 3.14(\mathrm{~d}, J=12.3 \mathrm{~Hz}, 1 \mathrm{H})$, $2.74-2.64(\mathrm{~m}, 1 \mathrm{H}), 2.55(\mathrm{~s}, 9 \mathrm{H}), 2.39-2.31(\mathrm{~m}, 2 \mathrm{H}), 2.00-1.84(\mathrm{~m}, 3 \mathrm{H}), 1.63-1.38$ $(\mathrm{m}, 2 \mathrm{H})$.

\subsection{Labeling experiment using alkenyl triflate and deuterated alkenyl triflate} (with one terminal D)

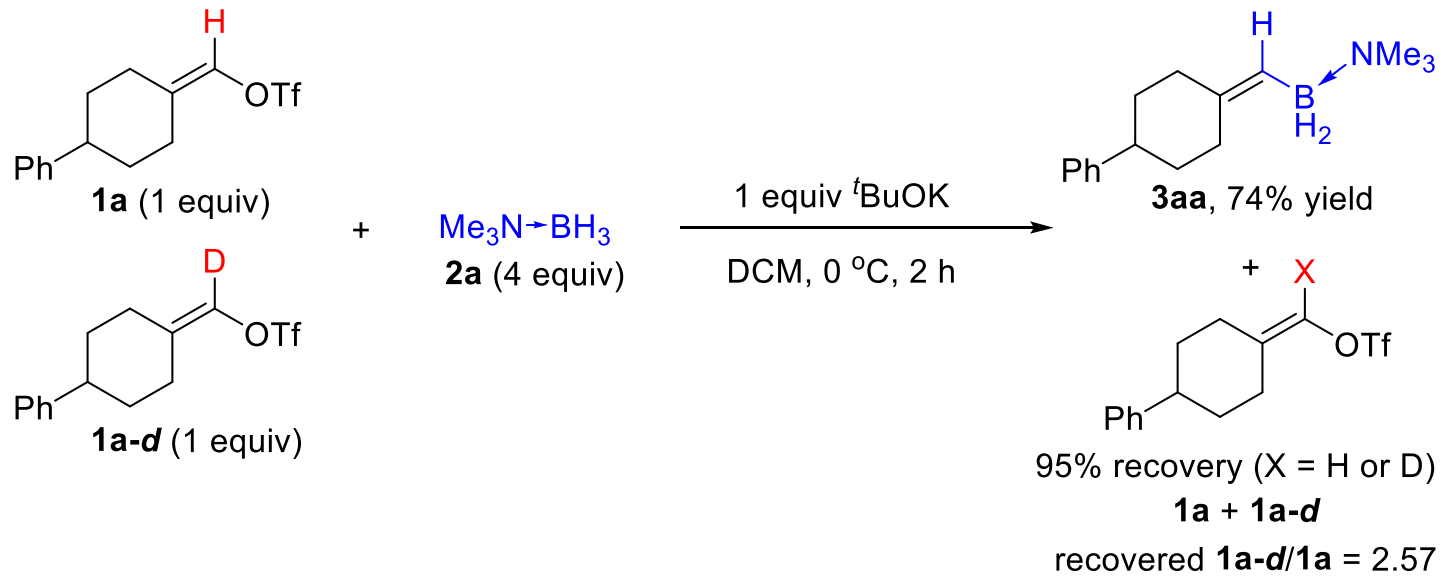


Under an argon atmosphere, $\mathbf{2 a}(87.5 \mathrm{mg}, 1.2 \mathrm{mmol})$ and ${ }^{t} \mathrm{BuOK}(33.7 \mathrm{mg}, 0.3 \mathrm{mmol})$ were introduced into a Schlenk tube. Then $\mathrm{CH}_{2} \mathrm{Cl}_{2}(2 \mathrm{~mL})$ was injected into the Schlenk tube in one portion at $0{ }^{\circ} \mathrm{C}$. Alkenyl triflate $1 \mathrm{a}(96.1 \mathrm{mg}, 0.3 \mathrm{mmol})$ and 1a-d was dissolved in $1 \mathrm{~mL} \mathrm{CH}_{2} \mathrm{Cl}_{2}$, and then was added into this reaction mixture in one portion. The resulting mixture was stirred at $0{ }^{\circ} \mathrm{C}$ for $2 \mathrm{~h}$. The remaining substrate and product were purified by flash column chromatography on silica gel $\left(\mathrm{PE} / \mathrm{EA} / \mathrm{Et}_{3} \mathrm{~N}=15: 1: 0.05\right.$, $v / v / v)$ to afford 3aa $(53.9 \mathrm{mg}, 74 \%)$ and $\mathbf{1 a}+\mathbf{1 a}-\boldsymbol{d}(91.2 \mathrm{mg}, 95 \%$ recovery). The deuterium distribution of the remaining substrate was determined by ${ }^{1} \mathrm{H}$ NMR of the crude product. ${ }^{1} \mathrm{H} \mathrm{NMR}\left(400 \mathrm{MHz}, \mathrm{CDCl}_{3}\right) \delta 7.33-7.26(\mathrm{~m}, 2 \mathrm{H}), 7.24-7.14(\mathrm{~m}, 3 \mathrm{H})$, $6.45(\mathrm{t}, J=2.1 \mathrm{~Hz}, 0.28 \mathrm{H}), 2.98-2.89(\mathrm{~m}, 1 \mathrm{H}), 2.70(\mathrm{tt}, J=12.3,3.4 \mathrm{~Hz}, 1 \mathrm{H}), 2.38-$ $2.27(\mathrm{~m}, 1 \mathrm{H}), 2.21-2.10(\mathrm{~m}, 1 \mathrm{H}), 2.10-2.01(\mathrm{~m}, 2 \mathrm{H}), 2.00-1.89(\mathrm{~m}, 1 \mathrm{H}), 1.61-$ $1.45(\mathrm{~m}, 2 \mathrm{H})$. 


\section{Computational Methods}

\section{$\underline{\text { DFT Methods }}$}

All DFT theoretical calculations have been carried out using the Gaussian 09 program package. The B3LYP ${ }^{8}$ method with $6-31+\mathrm{G}^{* 9}$ basis set has been selected for geometry optimizations and thermal energy correction calculations at $273 \mathrm{~K}$ temperature. Single-point calculations were performed at the M06-2X/6-311++G(2df, 2 p) ${ }^{10}$ level, including the D3 dispersion correction scheme developed by Grimme. ${ }^{11}$ The $\Delta \mathrm{G}_{s o l}$ and $\Delta \mathrm{H}_{s o l}$ values are retrieved from the addition of SCF energy to thermal correction of Gibbs free energy and enthalpy, respectively. All structures have been optimized considering solvent effects using the $\mathrm{SMD}^{12}$ model for DCM. Reaction paths were traced by the intrinsic reaction coordinate method for all transition states. All energetics reported throughout the text are in $\mathrm{kcal} / \mathrm{mol}$. Structures were generated using CYLview. ${ }^{13}$ The B3LYP/6-31+G(d,p) and B3LYP/6-31G(d) has been chosen for the the calculation of chemical shifts of NMR spectroscopy of $\mathbf{5 j}$ with scaling factors. ${ }^{14}$

\section{Complete Citation for Gaussian $\underline{09}$}

Gaussian 09, Revision D.01,

Frisch, M. J.; Trucks, G. W.; Schlegel, H. B.; Scuseria, G. E.; Robb, M. A.; Cheeseman, J. R.; Scalmani, G.; Barone, V.; Mennucci, B.; Petersson, G. A.; Nakatsuji, H.; Caricato, M.; Li, X.; Hratchian, H. P.; Izmaylov, A. F.; Bloino, J.; Zheng, G.; Sonnenberg, J. L.; Hada, M.; Ehara, M.; Toyota, K.; Fukuda, R.; Hasegawa, J.; Ishida, M.; Nakajima, T.; Honda, Y.; Kitao, O.; Nakai, H.; Vreven, T.; Montgomery, J. A., Jr.; Peralta, J. E.; Ogliaro, F.; Bearpark, M.; Heyd, J. J.; Brothers, E.; Kudin, K. N.; Staroverov, V. N.; Keith, T.; Kobayashi, R.; Normand, J.; Raghavachari, K.; Rendell, A.; Burant, J. C.; Iyengar, S. S.; Tomasi, J.; Cossi, M.; Rega, N.; Millam, J. M.; Klene, M.; Knox, J. E.; 
Cross, J. B.; Bakken, V.; Adamo, C.; Jaramillo, J.; Gomperts, R.; Stratmann, R. E.; Yazyev, O.; Austin, A. J.; Cammi, R.; Pomelli, C.; Ochterski, J. W.; Martin, R. L.; Morokuma, K.; Zakrzewski, V. G.; Voth, G. A.; Salvador, P.; Dannenberg, J. J.; Dapprich, S.; Daniels, A. D.; Farkas, O.; Foresman, J. B.; Ortiz, J. V.; Cioslowski, J.; Fox, D. J. Gaussian 09, Revision D.01; Gaussian, Inc.: Wallingford, CT, 2013.

Table S3. Sum of computed energies of stationary points for Scheme 5.

\begin{tabular}{ccc}
\hline Structure & $\Delta \mathrm{G}_{\text {sol }} /$ a.u. & $\Delta \mathrm{H}_{\text {sol }} /$ a.u. \\
\hline 1c & -1234.43768018 & -1234.59280718 \\
$\left({ }^{t} \text { BuOK }\right)_{4}$ & -3331.20729185 & -3331.64639185 \\
INT1 & -4565.63200799 & -4566.23902399 \\
TS1 & -4565.62320236 & -4566.22497736 \\
INT2 & -4565.63087618 & -4566.22949418 \\
BP & -4293.31821249 & -4293.77821149 \\
CB & -272.32467459 & -272.44737559 \\
2a & -200.95103399 & -200.82074199 \\
TS2 & -473.14160609 & -473.41539909 \\
3ca & -473.52681960 & -473.24898860 \\
\hline
\end{tabular}

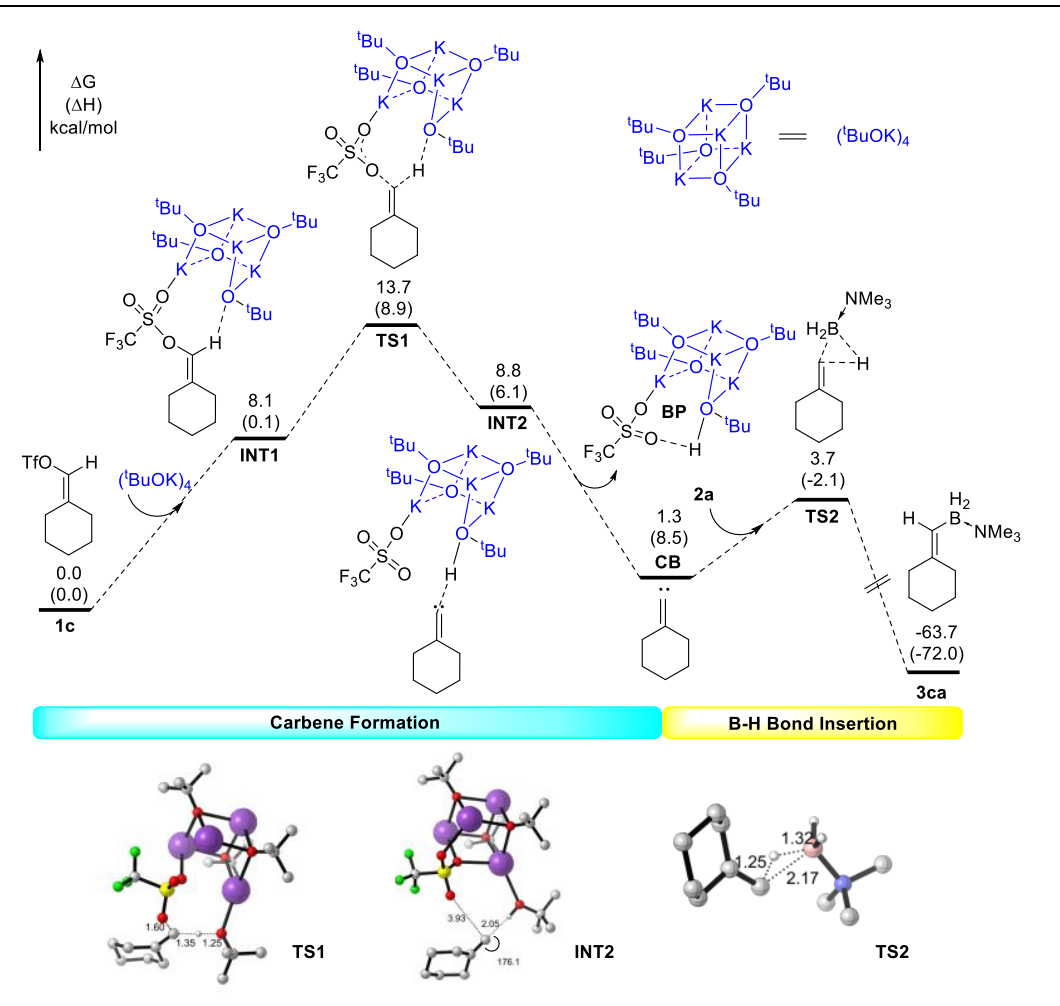


The Cartesian coordinates $(\AA)$ and energies at $273 \mathrm{~K}$ for the optimized structures

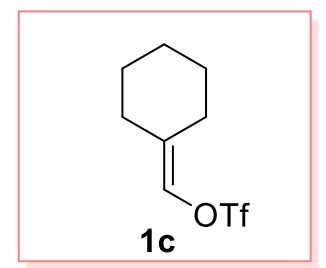

SCF energy[M062X-D3/6-311+G(2df,2p)/SMD(DCM)]: -1234.80045818 a.u.

Thermal correction to Enthalpy at $273 \mathrm{~K}: 0.207651$ a.u.

Thermal correction to Gibbs free energy at $273 \mathrm{~K}: 0.155127$ a.u.

01

$\mathrm{C}$

C

$\mathrm{C}$

$\mathrm{C}$

$\mathrm{C}$

$\mathrm{H}$

$\mathrm{H}$

$\mathrm{H}$

$\mathrm{H}$

$\mathrm{H}$

$\mathrm{H}$

$\mathrm{H}$

$\mathrm{H}$

$\mathrm{H}$

$\mathrm{H}$

C

C

$\mathrm{H}$

S

$\mathrm{O}$
$-2.14066900$

0.10021000

1.33860300

$-2.95083000$

$-1.27563900$

$-0.65854500$

$-3.75798700$

$-0.06023500$

$-1.16185100$

$-4.17321500$

0.8578830

$-0.00169100$

$-2.95700000$

1.30561800

0.82342600

$-2.60241600$

$-1.88575900$

$-1.50050400$

$-2.75182200$

$-0.45273500$

2.06962400

$-1.24313700$

0.43553300

1.86577300

$-3.14420700$

0.50720000

$-1.87590200$

$-4.64180100$

$-0.41028600$

$-1.71029300$

$-4.70926700$

1.73385000

$-0.38905300$

$-4.87937000$

0.32242500

0.65128600

$-2.30803200$

1.93897800

0.20192500

$-3.27635300$

1.91954700

1.67498300

$-3.6158250$

$-1.91499100$

$-0.05652800$

$-1.79015000$

$-0.83559200$

0.20681400

$-0.55812800$

$-1.26649800$

$-0.06440300$

$-0.27863200$

$-1.93588300$

$-0.86907900$

2.02075500

$-0.77175000$

0.19082100

2.16627600

$-1.56367200$

$-1.02151500$ 


$\begin{array}{lrrr}\mathrm{O} & 2.92012500 & -0.86933500 & 1.32237400 \\ \mathrm{C} & 2.00630200 & 1.04113300 & -0.34169600 \\ \mathrm{~F} & 3.20734900 & 1.35023000 & -0.83673000 \\ \mathrm{~F} & 1.07505500 & 1.23333700 & -1.28315600 \\ \mathrm{~F} & 1.74120800 & 1.82528400 & 0.70592200 \\ \mathrm{O} & 0.51814000 & -0.91584600 & 0.79493700\end{array}$

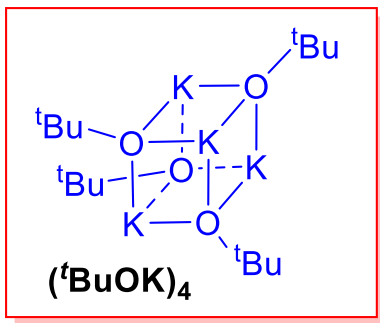

SCF energy[M062X-D3/6-311+G(2df,2p)/SMD(DCM)]: -3332.17923785 a.u.

Thermal correction to Enthalpy at $273 \mathrm{~K}: 0.532846$ a.u.

Thermal correction to Gibbs free energy at $273 \mathrm{~K}: 0.439100$ a.u.

01

$\mathrm{O}$

$\mathrm{O}$

$\mathrm{O}$

$\mathrm{O}$

K

K

K

K

$\mathrm{C}$

$\mathrm{C}$

C

$\mathrm{C}$

$\mathrm{C}$

$\mathrm{H}$

1.34918600

1.34918600

1.34918600

$\mathrm{O}$

$\begin{array}{lll}-1.34918600 & -1.34918600 & 1.34918600\end{array}$

O

$\begin{array}{lll}1.34918600 & -1.34918600 & -1.34918600\end{array}$

O

$\begin{array}{lll}-1.34918600 & 1.34918600 & -1.34918600\end{array}$

$\mathrm{K}$

$\begin{array}{lll}-1.30778900 & 1.30778900 & 1.30778900\end{array}$

$\mathrm{K}$

$1.30778900 \quad-1.30778900 \quad 1.30778900$

$\begin{array}{lll}1.30778900 & 1.30778900 & -1.30778900\end{array}$

$\mathrm{K}$

$\begin{array}{lll}-1.30778900 & -1.30778900 & -1.30778900\end{array}$

(1)

$\begin{array}{lll}2.15192200 & 2.15192200 \quad 2.15192200\end{array}$

$-2.15192200$

$-2.15192200$

2.15192200

2.15192200

$-2.15192200$

$-2.15192200$

$\mathrm{C}$

$-2.15192200$

2.15192200

$-2.15192200$

3.06130500

3.06130500

1.28378300

$\mathrm{H}$

2.44386700

3.70501100

0.64141800

3.71375400

3.71375400

1.88057400 


\begin{tabular}{|c|c|c|c|}
\hline $\mathrm{H}$ & 3.70501100 & 2.44386700 & 0.64141800 \\
\hline $\mathrm{C}$ & 3.06130500 & 1.28378300 & 3.06130500 \\
\hline $\mathrm{H}$ & 3.71375400 & 1.88057400 & 3.71375400 \\
\hline $\mathrm{H}$ & 2.44386700 & 0.64141800 & 3.70501100 \\
\hline $\mathrm{H}$ & 3.70501100 & 0.64141800 & 2.44386700 \\
\hline $\mathrm{C}$ & 1.28378300 & 3.06130500 & 3.06130500 \\
\hline $\mathrm{H}$ & 0.64141800 & 3.70501100 & 2.44386700 \\
\hline $\mathrm{H}$ & 0.64141800 & 2.44386700 & 3.70501100 \\
\hline $\mathrm{H}$ & 1.88057400 & 3.71375400 & 3.71375400 \\
\hline $\mathrm{C}$ & -1.28378300 & -3.06130500 & 3.06130500 \\
\hline $\mathrm{H}$ & -0.64141800 & -2.44386700 & 3.70501100 \\
\hline $\mathrm{H}$ & -1.88057400 & -3.71375400 & 3.71375400 \\
\hline $\mathrm{H}$ & -0.64141800 & -3.70501100 & 2.44386700 \\
\hline $\mathrm{C}$ & -3.06130500 & -3.06130500 & 1.28378300 \\
\hline $\mathrm{H}$ & -3.71375400 & -3.71375400 & 1.88057400 \\
\hline $\mathrm{H}$ & -3.70501100 & -2.44386700 & 0.64141800 \\
\hline $\mathrm{H}$ & -2.44386700 & -3.70501100 & 0.64141800 \\
\hline $\mathrm{C}$ & -3.06130500 & -1.28378300 & 3.06130500 \\
\hline $\mathrm{H}$ & -3.70501100 & -0.64141800 & 2.44386700 \\
\hline $\mathrm{H}$ & -3.71375400 & -1.88057400 & 3.71375400 \\
\hline $\mathrm{H}$ & -2.44386700 & -0.64141800 & 3.70501100 \\
\hline $\mathrm{C}$ & -3.06130500 & 3.06130500 & -1.28378300 \\
\hline $\mathrm{H}$ & -2.44386700 & 3.70501100 & -0.64141800 \\
\hline $\mathrm{H}$ & -3.71375400 & 3.71375400 & -1.88057400 \\
\hline $\mathrm{H}$ & -3.70501100 & 2.44386700 & -0.64141800 \\
\hline $\mathrm{C}$ & -1.28378300 & 3.06130500 & -3.06130500 \\
\hline $\mathrm{H}$ & -0.64141800 & 2.44386700 & -3.70501100 \\
\hline $\mathrm{H}$ & -1.88057400 & 3.71375400 & -3.71375400 \\
\hline $\mathrm{H}$ & -0.64141800 & 3.70501100 & -2.44386700 \\
\hline
\end{tabular}




$\begin{array}{lrrr}\mathrm{C} & -3.06130500 & 1.28378300 & -3.06130500 \\ \mathrm{H} & -2.44386700 & 0.64141800 & -3.70501100 \\ \mathrm{H} & -3.70501100 & 0.64141800 & -2.44386700 \\ \mathrm{H} & -3.71375400 & 1.88057400 & -3.71375400 \\ \mathrm{C} & 3.06130500 & -1.28378300 & -3.06130500 \\ \mathrm{H} & 3.71375400 & -1.88057400 & -3.71375400 \\ \mathrm{H} & 2.44386700 & -0.64141800 & -3.70501100 \\ \mathrm{H} & 3.70501100 & -0.64141800 & -2.44386700 \\ \mathrm{C} & 3.06130500 & -3.06130500 & -1.28378300 \\ \mathrm{H} & 2.44386700 & -3.70501100 & -0.64141800 \\ \mathrm{H} & 3.71375400 & -3.71375400 & -1.88057400 \\ \mathrm{H} & 3.70501100 & -2.44386700 & -0.64141800 \\ \mathrm{C} & 1.28378300 & -3.06130500 & -3.06130500 \\ \mathrm{H} & 0.64141800 & -3.70501100 & -2.44386700 \\ \mathrm{H} & 0.64141800 & -2.44386700 & -3.70501100 \\ \mathrm{H} & 1.88057400 & -3.71375400 & -3.71375400\end{array}$

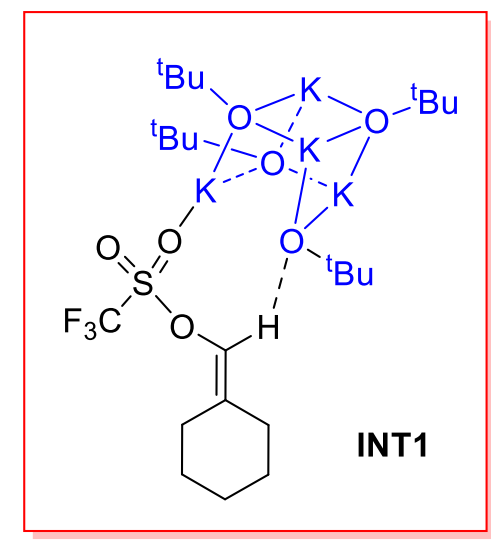

SCF energy[M062X-D3/6-311+G(2df,2p)/SMD(DCM)]: -4566.98039899 a.u.

Thermal correction to Enthalpy at $273 \mathrm{~K}: 0.741375$ a.u.

Thermal correction to Gibbs free energy at $273 \mathrm{~K}: 0.607016$ a.u.

01
$\mathrm{C}$
$-4.84318500$
1.98399800
$-0.24513700$
$\mathrm{C}$
$-2.72808000$
3.26015400
$-0.89816400$ 


\begin{tabular}{|c|c|c|c|}
\hline $\mathrm{C}$ & -2.87454000 & 4.14044700 & 0.35971500 \\
\hline $\mathrm{C}$ & -4.34212500 & 4.25692900 & 0.79811700 \\
\hline $\mathrm{C}$ & -4.97545000 & 2.87318600 & 1.01051000 \\
\hline $\mathrm{H}$ & -1.67448600 & 3.12177600 & -1.16417100 \\
\hline $\mathrm{H}$ & -5.45314000 & 2.42278500 & -1.05108600 \\
\hline $\mathrm{H}$ & -5.24252900 & 0.98396100 & -0.05555500 \\
\hline $\mathrm{H}$ & -2.28316800 & 3.70455800 & 1.17834600 \\
\hline $\mathrm{H}$ & -2.45231800 & 5.13435600 & 0.16390200 \\
\hline $\mathrm{H}$ & -4.41627100 & 4.85102300 & 1.71816700 \\
\hline $\mathrm{H}$ & -4.90878100 & 4.80139000 & 0.02734000 \\
\hline $\mathrm{H}$ & -4.48412400 & 2.37228900 & 1.85740900 \\
\hline $\mathrm{H}$ & -6.03554900 & 2.97305100 & 1.27599900 \\
\hline $\mathrm{H}$ & -3.20384400 & 3.77875900 & -1.74600500 \\
\hline $\mathrm{C}$ & -3.41068400 & 1.92047900 & -0.71792500 \\
\hline $\mathrm{C}$ & -2.72967000 & 0.80837400 & -1.00017100 \\
\hline $\mathrm{H}$ & -1.70552500 & 0.71940600 & -1.39800800 \\
\hline $\mathrm{S}$ & -2.81480800 & -1.61711400 & -0.04292600 \\
\hline $\mathrm{O}$ & -2.51471600 & -1.10443600 & 1.29620900 \\
\hline $\mathrm{O}$ & -1.82999200 & -2.42208100 & -0.75250800 \\
\hline $\mathrm{C}$ & -4.39621200 & -2.63567000 & 0.07625700 \\
\hline $\mathrm{F}$ & -4.11909200 & -3.73434800 & 0.78334500 \\
\hline $\mathrm{F}$ & -5.34676400 & -1.93529200 & 0.69918900 \\
\hline $\mathrm{F}$ & -4.81532500 & -2.97991400 & -1.14015500 \\
\hline $\mathrm{O}$ & -3.43636100 & -0.45399400 & -0.96204600 \\
\hline $\mathrm{O}$ & 3.62516500 & -0.31824800 & -0.94572700 \\
\hline $\mathrm{O}$ & 1.78544100 & 1.93500900 & 1.56210200 \\
\hline $\mathrm{O}$ & 1.32298400 & -1.74824200 & 1.70545900 \\
\hline $\mathrm{O}$ & 0.07193500 & 0.42988200 & -2.22234400 \\
\hline K & 2.06253600 & 1.80438400 & -1.13840400 \\
\hline
\end{tabular}




\begin{tabular}{|c|c|c|c|}
\hline K & 3.45061700 & -0.18697500 & 1.65495300 \\
\hline $\mathrm{K}$ & 1.27370000 & -1.71142800 & -1.03609400 \\
\hline K & -0.21700500 & 0.30376100 & 2.05513700 \\
\hline $\mathrm{C}$ & 4.80191300 & -0.62851300 & -1.61867100 \\
\hline $\mathrm{C}$ & 1.98036900 & 3.12666600 & 2.25478200 \\
\hline $\mathrm{C}$ & 1.27395500 & -2.94159200 & 2.42385400 \\
\hline $\mathrm{C}$ & -0.00733700 & 0.39983900 & -3.61668500 \\
\hline $\mathrm{C}$ & 5.22923000 & 0.53902600 & -2.54656000 \\
\hline $\mathrm{H}$ & 5.38036600 & 1.45187400 & -1.95309200 \\
\hline $\mathrm{H}$ & 6.16152100 & 0.33515500 & -3.09182000 \\
\hline $\mathrm{H}$ & 4.44403200 & 0.73560200 & -3.28870200 \\
\hline $\mathrm{C}$ & 4.61911900 & -1.90031700 & -2.48729400 \\
\hline $\mathrm{H}$ & 5.52904700 & -2.17934800 & -3.03693500 \\
\hline $\mathrm{H}$ & 4.33906500 & -2.75130900 & -1.85057500 \\
\hline $\mathrm{H}$ & 3.81986800 & -1.73926500 & -3.22280300 \\
\hline $\mathrm{C}$ & 5.95736700 & -0.89304400 & -0.61507800 \\
\hline $\mathrm{H}$ & 6.13149200 & 0.00026300 & 0.00265300 \\
\hline $\mathrm{H}$ & 5.69654400 & -1.73172300 & 0.04628200 \\
\hline $\mathrm{H}$ & 6.90693200 & -1.14309300 & -1.10819400 \\
\hline $\mathrm{C}$ & 3.25711600 & 3.04728000 & 3.13312800 \\
\hline $\mathrm{H}$ & 4.13729700 & 2.86439500 & 2.50096800 \\
\hline $\mathrm{H}$ & 3.44381500 & 3.96690800 & 3.70477000 \\
\hline $\mathrm{H}$ & 3.16730800 & 2.22047300 & 3.85193700 \\
\hline $\mathrm{C}$ & 0.77467200 & 3.42961200 & 3.18461000 \\
\hline $\mathrm{H}$ & 0.88485600 & 4.37152400 & 3.73923600 \\
\hline $\mathrm{H}$ & -0.14795100 & 3.49809100 & 2.59135500 \\
\hline $\mathrm{H}$ & 0.65589600 & 2.62572300 & 3.92607000 \\
\hline $\mathrm{C}$ & 2.14155800 & 4.31907700 & 1.27523200 \\
\hline $\mathrm{H}$ & 1.24846000 & 4.40849700 & 0.64154100 \\
\hline
\end{tabular}




\begin{tabular}{|c|c|c|c|}
\hline $\mathrm{H}$ & 2.28475600 & 5.27951300 & 1.78900100 \\
\hline $\mathrm{H}$ & 3.01555100 & 4.15989900 & 0.62772900 \\
\hline $\mathrm{C}$ & 0.38250100 & -0.99967200 & -4.16656100 \\
\hline $\mathrm{H}$ & -0.27808000 & -1.76535600 & -3.73716700 \\
\hline $\mathrm{H}$ & 0.29949300 & -1.06420100 & -5.26029500 \\
\hline $\mathrm{H}$ & 1.42329000 & -1.23850200 & -3.90629600 \\
\hline $\mathrm{C}$ & -1.43912800 & 0.71840300 & -4.12064700 \\
\hline $\mathrm{H}$ & -1.75450000 & 1.70984700 & -3.77162300 \\
\hline $\mathrm{H}$ & -1.50223600 & 0.70987200 & -5.21775500 \\
\hline $\mathrm{H}$ & -2.15419200 & -0.01953300 & -3.73713900 \\
\hline $\mathrm{C}$ & 0.95472500 & 1.44264900 & -4.25142900 \\
\hline $\mathrm{H}$ & 0.70600400 & 2.45070200 & -3.88878600 \\
\hline $\mathrm{H}$ & 1.99767400 & 1.21767200 & -3.98753300 \\
\hline $\mathrm{H}$ & 0.89552400 & 1.46243600 & -5.34842800 \\
\hline $\mathrm{C}$ & 0.14782500 & -2.89612600 & 3.49004700 \\
\hline $\mathrm{H}$ & 0.07733000 & -3.82212900 & 4.07740700 \\
\hline $\mathrm{H}$ & 0.33281900 & -2.07182400 & 4.19486500 \\
\hline $\mathrm{H}$ & -0.82338300 & -2.72919600 & 3.00698900 \\
\hline $\mathrm{C}$ & 1.00402700 & -4.14281800 & 1.48115100 \\
\hline $\mathrm{H}$ & 1.81523600 & -4.23271900 & 0.74441000 \\
\hline $\mathrm{H}$ & 0.94030200 & -5.09970300 & 2.01747700 \\
\hline $\mathrm{H}$ & 0.05896500 & -3.99364100 & 0.94340600 \\
\hline $\mathrm{C}$ & 2.61886500 & -3.19537500 & 3.15618500 \\
\hline $\mathrm{H}$ & 3.44064500 & -3.25066500 & 2.42841800 \\
\hline $\mathrm{H}$ & 2.82722900 & -2.37489500 & 3.85862600 \\
\hline $\mathrm{H}$ & 2.62256300 & -4.13109000 & 3.73207200 \\
\hline
\end{tabular}




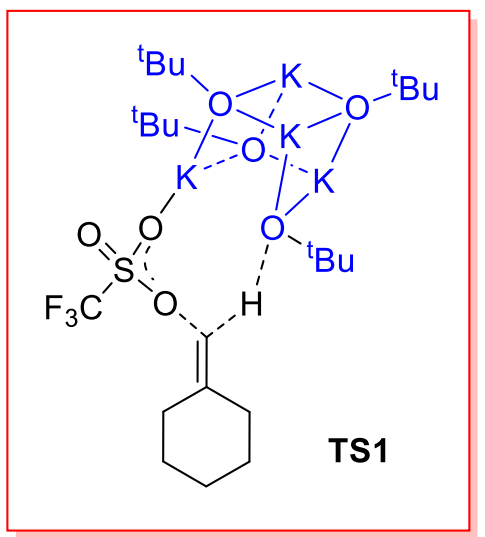

SCF energy[M062X-D3/6-311+G(2df,2p)/SMD(DCM)]: -4566.96076836 a.u.

Thermal correction to Enthalpy at $273 \mathrm{~K}: 0.735791$ a.u.

Thermal correction to Gibbs free energy at $273 \mathrm{~K}: 0.601775$ a.u.

01

$\mathrm{C}$

$\mathrm{C}$

$\mathrm{C}$

$\mathrm{C}$

$\mathrm{C}$

$\mathrm{H}$

$\mathrm{H}$

$\mathrm{H}$

$\mathrm{H}$

H

$\mathrm{H}$

H

H

H

H

C

C

$\mathrm{H}$

$\begin{array}{rrr}-5.25014300 & -1.37933400 & -0.29470200 \\ -5.12573300 & 0.64889600 & 1.24254200 \\ -5.44235700 & -0.25341000 & 2.45195700 \\ -6.27387000 & -1.47716500 & 2.03944700 \\ -5.58338000 & -2.27195400 & 0.92011600 \\ -4.48578200 & 1.48522200 & 1.54021900 \\ -6.19633800 & -1.05178200 & -0.75577100 \\ -4.71331100 & -1.94916400 & -1.05725300 \\ -4.49828800 & -0.58830900 & 2.90650300 \\ -5.97301700 & 0.32877900 & 3.21713300 \\ -6.45452900 & -2.12579300 & 2.90708900 \\ -7.26203800 & -1.14138900 & 1.68870400 \\ -4.65267100 & -2.71103500 & 1.30785500 \\ -6.21742900 & -3.10862200 & 0.59761000 \\ -6.06818700 & 1.08234100 & 0.86959600 \\ -4.47693100 & -0.14744700 & 0.12023100 \\ -3.32104500 & 0.33050700 & -0.34618500 \\ -2.64440100 & 1.50288600 & -0.31661700\end{array}$




\begin{tabular}{|c|c|c|c|}
\hline $\mathrm{S}$ & -1.38083700 & -1.12109500 & -1.61451400 \\
\hline $\mathrm{O}$ & -0.55668300 & -0.79270200 & -0.43394700 \\
\hline $\mathrm{O}$ & -0.74900800 & -0.94961200 & -2.92480800 \\
\hline $\mathrm{C}$ & -1.69610000 & -2.97277800 & -1.46437500 \\
\hline $\mathrm{F}$ & -0.52750500 & -3.62478600 & -1.59415300 \\
\hline $\mathrm{F}$ & -2.20664600 & -3.25805900 & -0.25520500 \\
\hline $\mathrm{F}$ & -2.53864800 & -3.39283300 & -2.40739700 \\
\hline $\mathrm{O}$ & -2.81180800 & -0.55566000 & -1.57729500 \\
\hline $\mathrm{O}$ & 2.67037200 & 1.71498900 & -0.98200200 \\
\hline $\mathrm{O}$ & 1.59709300 & 0.53594500 & 2.52491900 \\
\hline $\mathrm{O}$ & 2.97712600 & -1.91218600 & -0.02038400 \\
\hline $\mathrm{O}$ & -1.98480700 & 2.56946900 & -0.30181800 \\
\hline K & 0.40914300 & 2.02833600 & 0.54214800 \\
\hline K & 3.77726600 & 0.43847900 & 1.03548300 \\
\hline $\mathrm{K}$ & 2.05253700 & -0.54812000 & -2.11201100 \\
\hline K & 0.93662600 & -1.80540200 & 1.63716900 \\
\hline $\mathrm{C}$ & 3.27363200 & 2.76025600 & -1.68110400 \\
\hline $\mathrm{C}$ & 1.50351200 & 0.92285800 & 3.86082200 \\
\hline $\mathrm{C}$ & 3.77285700 & -3.04709900 & -0.16987100 \\
\hline $\mathrm{C}$ & -2.57470800 & 3.65438600 & -0.98956600 \\
\hline $\mathrm{C}$ & 2.49147500 & 4.08129900 & -1.47922300 \\
\hline $\mathrm{H}$ & 2.46480200 & 4.34337200 & -0.41225400 \\
\hline $\mathrm{H}$ & 2.93742900 & 4.92849100 & -2.01814900 \\
\hline $\mathrm{H}$ & 1.45913600 & 3.96539000 & -1.83265900 \\
\hline $\mathrm{C}$ & 3.31901800 & 2.45246900 & -3.20151900 \\
\hline $\mathrm{H}$ & 3.77552300 & 3.26101700 & -3.78833200 \\
\hline $\mathrm{H}$ & 3.90851500 & 1.54199000 & -3.38793600 \\
\hline $\mathrm{H}$ & 2.30089400 & 2.29834800 & -3.58476300 \\
\hline $\mathrm{C}$ & 4.72964700 & 2.97730200 & -1.19043100 \\
\hline
\end{tabular}




\begin{tabular}{|c|c|c|c|}
\hline $\mathrm{H}$ & 4.73278300 & 3.23326100 & -0.12109400 \\
\hline $\mathrm{H}$ & 5.31731200 & 2.05878700 & -1.33228900 \\
\hline $\mathrm{H}$ & 5.24513600 & 3.78751700 & -1.72401300 \\
\hline $\mathrm{C}$ & 2.91274600 & 1.19500600 & 4.44867400 \\
\hline $\mathrm{H}$ & 3.40445100 & 2.00186200 & 3.88705800 \\
\hline $\mathrm{H}$ & 2.88648600 & 1.49521600 & 5.50526000 \\
\hline $\mathrm{H}$ & 3.53293200 & 0.29055200 & 4.37287400 \\
\hline $\mathrm{C}$ & 0.83255900 & -0.19095600 & 4.70793700 \\
\hline $\mathrm{H}$ & 0.72992600 & 0.07762100 & 5.76809100 \\
\hline $\mathrm{H}$ & -0.17415000 & -0.40194300 & 4.32026700 \\
\hline $\mathrm{H}$ & 1.42966600 & -1.11388700 & 4.65947000 \\
\hline $\mathrm{C}$ & 0.65876500 & 2.21424500 & 4.00319200 \\
\hline $\mathrm{H}$ & -0.35375100 & 2.05132600 & 3.61025400 \\
\hline $\mathrm{H}$ & 0.56285300 & 2.54873900 & 5.04512100 \\
\hline $\mathrm{H}$ & 1.12432400 & 3.03208400 & 3.43550100 \\
\hline $\mathrm{C}$ & -2.57107200 & 3.38555900 & -2.51143400 \\
\hline $\mathrm{H}$ & -3.14692300 & 2.48124600 & -2.74172700 \\
\hline $\mathrm{H}$ & -3.00521600 & 4.22076300 & -3.07847200 \\
\hline $\mathrm{H}$ & -1.54372500 & 3.22918100 & -2.86372100 \\
\hline $\mathrm{C}$ & -4.02629600 & 3.88251100 & -0.51136200 \\
\hline $\mathrm{H}$ & -4.04212700 & 4.05502000 & 0.57188500 \\
\hline $\mathrm{H}$ & -4.48346200 & 4.75065000 & -1.00519600 \\
\hline $\mathrm{H}$ & -4.64725600 & 3.00549300 & -0.72649300 \\
\hline $\mathrm{C}$ & -1.75005300 & 4.92221300 & -0.69190300 \\
\hline $\mathrm{H}$ & -1.73578100 & 5.11707400 & 0.38815400 \\
\hline $\mathrm{H}$ & -0.71475700 & 4.79759400 & -1.03431100 \\
\hline $\mathrm{H}$ & -2.16339800 & 5.80769800 & -1.19344500 \\
\hline $\mathrm{C}$ & 3.19512800 & -4.23721300 & 0.64111500 \\
\hline $\mathrm{H}$ & 3.79177800 & -5.15394600 & 0.54124100 \\
\hline
\end{tabular}




$\begin{array}{lrrr}\mathrm{H} & 3.16071800 & -3.98471300 & 1.71116200 \\ \mathrm{H} & 2.17563100 & -4.46593900 & 0.30001100 \\ \mathrm{C} & 3.85096100 & -3.47469600 & -1.65893100 \\ \mathrm{H} & 4.29838600 & -2.67021200 & -2.26079000 \\ \mathrm{H} & 4.46062700 & -4.37502900 & -1.81394600 \\ \mathrm{H} & 2.84362700 & -3.68369700 & -2.04496300 \\ \mathrm{C} & 5.21412800 & -2.77338100 & 0.33256700 \\ \mathrm{H} & 5.65888800 & -1.94493400 & -0.23626100 \\ \mathrm{H} & 5.19521700 & -2.49461300 & 1.39561100 \\ \mathrm{H} & 5.87750600 & -3.64285400 & 0.22851700\end{array}$

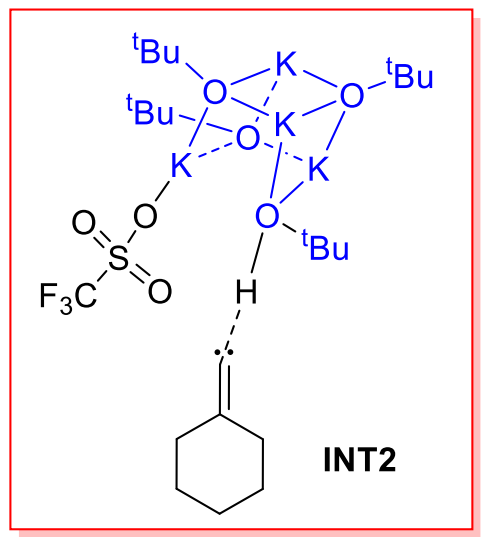

SCF energy[M062X-D3/6-311+G(2df,2p)/SMD(DCM)]: -4566.97048118 a.u.

Thermal correction to Enthalpy at 273 K: 0.740987 a.u.

Thermal correction to Gibbs free energy at $273 \mathrm{~K}: 0.598618$ a.u.

01

$\begin{array}{lrrr}\mathrm{C} & -5.40474700 & -1.36855300 & -1.25560200 \\ \mathrm{C} & -6.81119500 & -0.30405700 & 0.66205200 \\ \mathrm{C} & -6.88896300 & -1.68597400 & 1.33591600 \\ \mathrm{C} & -6.79422100 & -2.82736700 & 0.31094800 \\ \mathrm{C} & -5.51872600 & -2.72467200 & -0.53924600 \\ \mathrm{H} & -6.80139900 & 0.49801300 & 1.40716500 \\ \mathrm{H} & -6.20779900 & -1.25997600 & -1.99961000 \\ \mathrm{H} & -4.44702100 & -1.28988600 & -1.77424800\end{array}$




\begin{tabular}{|c|c|c|c|}
\hline $\mathrm{H}$ & -6.07175700 & -1.77548600 & 2.06455100 \\
\hline $\mathrm{H}$ & -7.82922500 & -1.75259300 & 1.89916000 \\
\hline $\mathrm{H}$ & -6.82272400 & -3.79454500 & 0.82853000 \\
\hline $\mathrm{H}$ & -7.67676500 & -2.79846500 & -0.34671600 \\
\hline $\mathrm{H}$ & -4.63241200 & -2.86482100 & 0.09216000 \\
\hline $\mathrm{H}$ & -5.49607800 & -3.52071500 & -1.29443400 \\
\hline $\mathrm{H}$ & -7.69356200 & -0.14663800 & 0.02472800 \\
\hline $\mathrm{C}$ & -5.57748000 & -0.23351100 & -0.23952200 \\
\hline $\mathrm{C}$ & -4.68748800 & 0.70374000 & -0.11816300 \\
\hline $\mathrm{H}$ & -3.26990600 & 2.17712700 & -0.06715100 \\
\hline $\mathrm{S}$ & -0.74613300 & -2.24313600 & -1.07521500 \\
\hline $\mathrm{O}$ & -0.10014000 & -1.20855200 & -0.19854000 \\
\hline $\mathrm{O}$ & 0.17287700 & -2.73723600 & -2.12672300 \\
\hline $\mathrm{C}$ & -0.90516200 & -3.69714100 & 0.10714100 \\
\hline $\mathrm{F}$ & 0.29362300 & -3.95708300 & 0.69395900 \\
\hline $\mathrm{F}$ & -1.77986600 & -3.42029800 & 1.09312600 \\
\hline $\mathrm{F}$ & -1.30954200 & -4.80581400 & -0.52090000 \\
\hline $\mathrm{O}$ & -2.12623500 & -1.94470100 & -1.47577300 \\
\hline $\mathrm{O}$ & 2.08052600 & 1.57408300 & -1.71920500 \\
\hline $\mathrm{O}$ & 1.56058800 & 1.22745300 & 2.07243400 \\
\hline $\mathrm{O}$ & 3.79067800 & -1.22470900 & 0.21483000 \\
\hline $\mathrm{O}$ & -2.52930800 & 2.82827700 & -0.06187900 \\
\hline $\mathrm{K}$ & -0.08345300 & 1.57730000 & -0.06212700 \\
\hline $\mathrm{K}$ & 3.62391800 & 1.43176800 & 0.42178200 \\
\hline $\mathrm{K}$ & 2.35899300 & -1.04913300 & -2.01818000 \\
\hline $\mathrm{K}$ & 1.72653400 & -1.38144300 & 1.84477500 \\
\hline $\mathrm{C}$ & 2.24152400 & 2.43845200 & -2.79927300 \\
\hline $\mathrm{C}$ & 1.43454200 & 1.88431000 & 3.29377700 \\
\hline $\mathrm{C}$ & 4.94012800 & -2.00372000 & 0.32493500 \\
\hline
\end{tabular}




\begin{tabular}{|c|c|c|c|}
\hline $\mathrm{C}$ & -3.07276500 & 4.16684800 & -0.10200200 \\
\hline $\mathrm{C}$ & 0.98976100 & 3.33422700 & -2.97950000 \\
\hline $\mathrm{H}$ & 0.82790900 & 3.93798000 & -2.07604000 \\
\hline $\mathrm{H}$ & 1.07608000 & 4.02448900 & -3.83025700 \\
\hline $\mathrm{H}$ & 0.10049200 & 2.71050100 & -3.14141100 \\
\hline $\mathrm{C}$ & 2.45862400 & 1.64465000 & -4.11566400 \\
\hline $\mathrm{H}$ & 2.57900500 & 2.29485400 & -4.99297400 \\
\hline $\mathrm{H}$ & 3.36541200 & 1.02627800 & -4.04043500 \\
\hline $\mathrm{H}$ & 1.59850000 & 0.98748000 & -4.30536800 \\
\hline $\mathrm{C}$ & 3.47162600 & 3.35984100 & -2.58483800 \\
\hline $\mathrm{H}$ & 3.33816200 & 3.95756900 & -1.67209100 \\
\hline $\mathrm{H}$ & 4.38167600 & 2.75287000 & -2.47371800 \\
\hline $\mathrm{H}$ & 3.63720800 & 4.05740600 & -3.41767800 \\
\hline $\mathrm{C}$ & 2.72215600 & 2.68682500 & 3.62073900 \\
\hline $\mathrm{H}$ & 2.90292200 & 3.44089000 & 2.84137100 \\
\hline $\mathrm{H}$ & 2.66678800 & 3.21380700 & 4.58340400 \\
\hline $\mathrm{H}$ & 3.58826100 & 2.01101100 & 3.65945700 \\
\hline $\mathrm{C}$ & 1.19522500 & 0.86830200 & 4.44252500 \\
\hline $\mathrm{H}$ & 1.07306500 & 1.34888200 & 5.42290100 \\
\hline $\mathrm{H}$ & 0.28602100 & 0.28438000 & 4.24175100 \\
\hline $\mathrm{H}$ & 2.04743300 & 0.17683900 & 4.52062600 \\
\hline $\mathrm{C}$ & 0.24170700 & 2.87314500 & 3.26618200 \\
\hline $\mathrm{H}$ & -0.69168900 & 2.33549800 & 3.05386000 \\
\hline $\mathrm{H}$ & 0.11237500 & 3.41265600 & 4.21485300 \\
\hline $\mathrm{H}$ & 0.39417400 & 3.61964100 & 2.47474500 \\
\hline $\mathrm{C}$ & -3.86967200 & 4.35665000 & -1.40251700 \\
\hline $\mathrm{H}$ & -4.71048500 & 3.65472100 & -1.44988900 \\
\hline $\mathrm{H}$ & -4.27225000 & 5.37494500 & -1.47197800 \\
\hline $\mathrm{H}$ & -3.22729000 & 4.17870100 & -2.27223500 \\
\hline
\end{tabular}




\begin{tabular}{|c|c|c|c|}
\hline $\mathrm{C}$ & -3.96839200 & 4.38973900 & 1.12747200 \\
\hline $\mathrm{H}$ & -3.39981300 & 4.22195400 & 2.04897100 \\
\hline $\mathrm{H}$ & -4.36515400 & 5.41241500 & 1.14559600 \\
\hline $\mathrm{H}$ & -4.81962400 & 3.69828900 & 1.11885100 \\
\hline $\mathrm{C}$ & -1.86591000 & 5.10780300 & -0.06544400 \\
\hline $\mathrm{H}$ & -1.28295600 & 4.95074100 & 0.84955900 \\
\hline $\mathrm{H}$ & -1.21545300 & 4.93410900 & -0.93038300 \\
\hline $\mathrm{H}$ & -2.18923300 & 6.15477100 & -0.08843000 \\
\hline $\mathrm{C}$ & 4.81224300 & -3.00856300 & 1.49998700 \\
\hline $\mathrm{H}$ & 5.70065200 & -3.64350400 & 1.61991800 \\
\hline $\mathrm{H}$ & 4.66409900 & -2.46572700 & 2.44477700 \\
\hline $\mathrm{H}$ & 3.95015100 & -3.67022400 & 1.33613300 \\
\hline $\mathrm{C}$ & 5.18445600 & -2.81218400 & -0.97626200 \\
\hline $\mathrm{H}$ & 5.31345500 & -2.12710100 & -1.82673600 \\
\hline $\mathrm{H}$ & 6.08118800 & -3.44476800 & -0.92527800 \\
\hline $\mathrm{H}$ & 4.32585800 & -3.46645200 & -1.18089900 \\
\hline $\mathrm{C}$ & 6.18179200 & -1.11065700 & 0.58188400 \\
\hline $\mathrm{H}$ & 6.30954400 & -0.39932000 & -0.24618800 \\
\hline $\mathrm{H}$ & 6.04898200 & -0.54049300 & 1.51209200 \\
\hline $\mathrm{H}$ & 7.11323500 & -1.68625800 & 0.67369800 \\
\hline
\end{tabular}

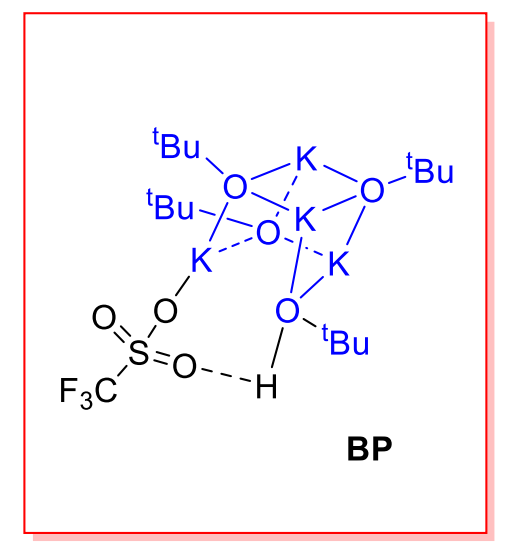

SCF energy[M062X-D3/6-311+G(2df,2p)/SMD(DCM)]: -4294.36030149 a.u.

Thermal correction to Enthalpy at 273 K: 0.582090 a.u. 
Thermal correction to Gibbs free energy at $273 \mathrm{~K}: 0.459999$ a.u.

01

$\mathrm{H}$

$3.56003500 \quad 0.30871500 \quad-0.10995700$

$\mathrm{S}$

$\begin{array}{lll}1.47705600 & 2.28424300 & -0.60833200\end{array}$

$\mathrm{O}$

$\begin{array}{lll}0.68652700 & 1.28057000 & 0.17857000\end{array}$

$\mathrm{O}$

$\begin{array}{lll}0.84959300 & 2.60969000 \quad-1.90693000\end{array}$

$\mathrm{C}$

1.27770600

3.84596300

0.41568500

F

$-0.03834100$

4.09276000

0.62048700

F

$\begin{array}{lll}1.85660900 \quad 3.69936600 & 1.62025300\end{array}$

F

1.81625100

4.90411500

$-0.19606200$

$\mathrm{O}$

2.93186200

2.03321600

$-0.61511700$

O

$-0.60015200$

$-1.82692300$

$-1.71734300$

$\mathrm{O}$

$-0.97077000$

$-1.45139800$

2.07340300

$\mathrm{O}$

$-3.07650400$

0.66949000

$-0.28138400$

$\mathrm{O}$

3.68384600

$-0.63470500$

0.12842000

K

1.10248900

$-1.50255600$

0.34062800

K

$-2.59016400$

$-1.93526300$

0.02540800

K

$-1.19260800$

0.71903700

$-2.16276500$

K

$-1.50090200$

1.10193800

1.78274900

C

$-0.37066000$

$-2.73908500$

$-2.74542800$

C

$-0.94695600$

$-2.12365000$

3.29295900

C

$-4.32463600$

1.26814300

$-0.44245100$

C

5.09503700

$-0.95174400$

0.08437000

$\mathrm{C}$

1.00353000

$-3.43556600$

$-2.56769900$

$\mathrm{H}$

1.02849700

$-3.98280700$

$-1.61430700$

$\mathrm{H}$

1.22417900

$-4.15626500$

$-3.36704600$

$\mathrm{H}$

$\begin{array}{lll}1.80660600 & -2.68647200 & -2.56029700\end{array}$

C

$-0.37657100$

$-2.02849400$

$-4.12469300$

$\mathrm{H}$

$-0.18805100$

$-2.71484400$

$-4.96146300$ 


\begin{tabular}{|c|c|c|c|}
\hline $\mathrm{H}$ & -1.35421700 & -1.55666500 & -4.30218100 \\
\hline $\mathrm{H}$ & 0.39889600 & -1.25064900 & -4.15108800 \\
\hline $\mathrm{C}$ & -1.47064500 & -3.83321000 & -2.76130200 \\
\hline $\mathrm{H}$ & -1.47695600 & -4.37441500 & -1.80434500 \\
\hline $\mathrm{H}$ & -2.45814500 & -3.37240900 & -2.90715600 \\
\hline $\mathrm{H}$ & -1.32892700 & -4.57447200 & -3.55992000 \\
\hline $\mathrm{C}$ & -2.08101700 & -3.18014800 & 3.35643700 \\
\hline $\mathrm{H}$ & -1.95442200 & -3.91621700 & 2.54981200 \\
\hline $\mathrm{H}$ & -2.10362000 & -3.73073100 & 4.30707600 \\
\hline $\mathrm{H}$ & -3.05720700 & -2.69094500 & 3.23051700 \\
\hline $\mathrm{C}$ & -1.14119600 & -1.13276600 & 4.47092600 \\
\hline $\mathrm{H}$ & -1.11923700 & -1.62346000 & 5.45354800 \\
\hline $\mathrm{H}$ & -0.34464300 & -0.37556300 & 4.45902500 \\
\hline $\mathrm{H}$ & -2.11154900 & -0.62319800 & 4.37923900 \\
\hline $\mathrm{C}$ & 0.40733400 & -2.85246400 & 3.49401700 \\
\hline $\mathrm{H}$ & 1.23256200 & -2.12730400 & 3.47377400 \\
\hline $\mathrm{H}$ & 0.46422300 & -3.39506300 & 4.44758000 \\
\hline $\mathrm{H}$ & 0.56488400 & -3.58310100 & 2.68789600 \\
\hline $\mathrm{C}$ & 5.61952700 & -0.72844700 & -1.34202800 \\
\hline $\mathrm{H}$ & 5.50264000 & 0.32052700 & -1.63958300 \\
\hline $\mathrm{H}$ & 6.68373300 & -0.98460900 & -1.41418000 \\
\hline $\mathrm{H}$ & 5.06547100 & -1.34932700 & -2.05501700 \\
\hline $\mathrm{C}$ & 5.84296500 & -0.06314400 & 1.08948800 \\
\hline $\mathrm{H}$ & 5.44115900 & -0.20584300 & 2.09895500 \\
\hline $\mathrm{H}$ & 6.91339500 & -0.30202000 & 1.10518600 \\
\hline $\mathrm{H}$ & 5.73726200 & 0.99606100 & 0.82544200 \\
\hline $\mathrm{C}$ & 5.19447600 & -2.42717500 & 0.47928900 \\
\hline $\mathrm{H}$ & 4.78566800 & -2.58565500 & 1.48423200 \\
\hline $\mathrm{H}$ & 4.63738100 & -3.05415000 & -0.22698900 \\
\hline
\end{tabular}




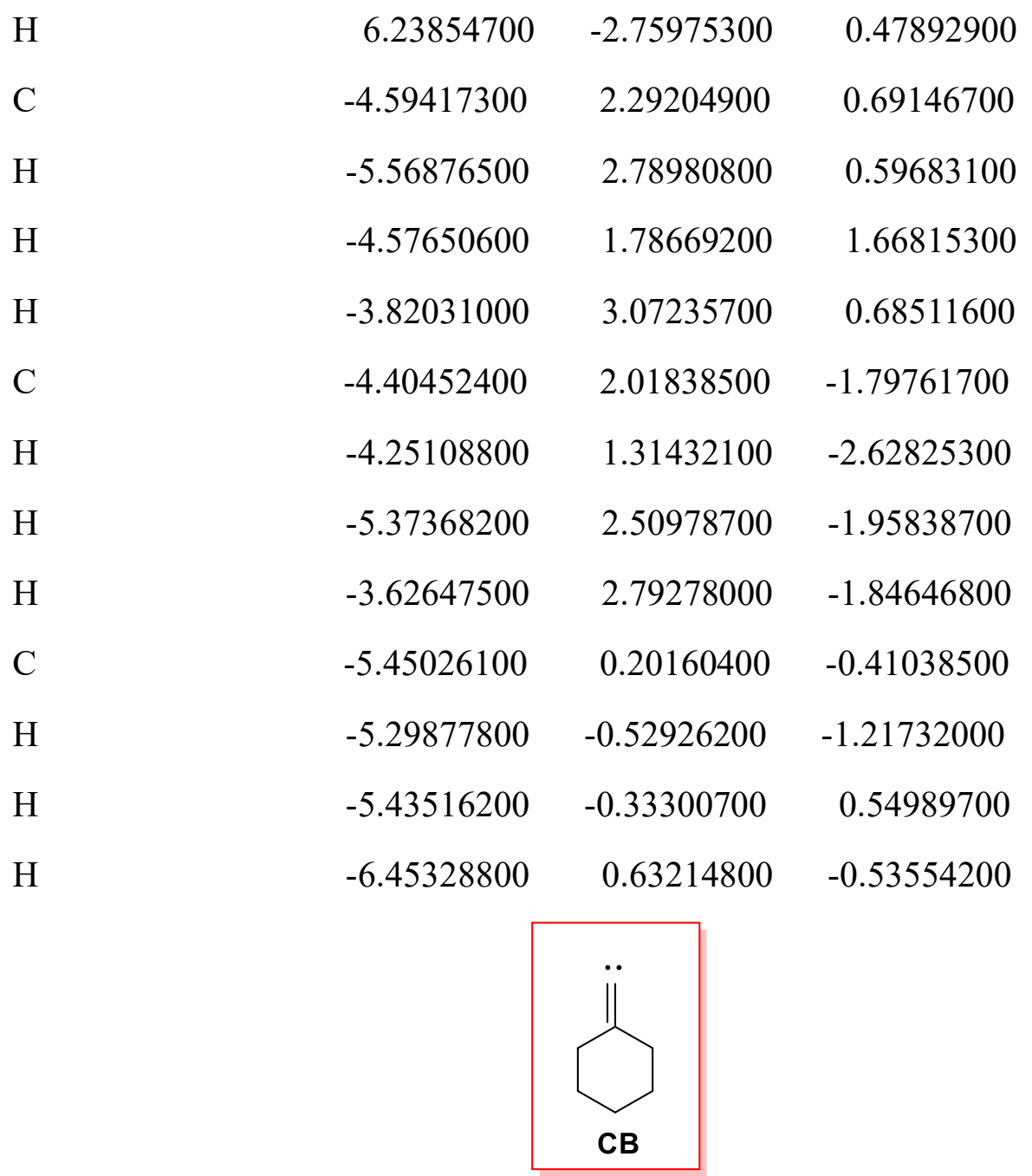

SCF energy[M062X-D3/6-311+G(2df,2p)/SMD(DCM)]: -272.60427459 a.u.

Thermal correction to Enthalpy at $273 \mathrm{~K}: 0.156899$ a.u.

Thermal correction to Gibbs free energy at $273 \mathrm{~K}: 0.122701$ a.u.

01

$\begin{array}{lrrr}\mathrm{C} & -0.47709000 & -1.29918600 & 0.37075000 \\ \mathrm{C} & -0.47709800 & 1.29918200 & 0.37075100 \\ \mathrm{C} & 0.91630700 & 1.27332000 & -0.28423700 \\ \mathrm{C} & 1.69566700 & 0.00000400 & 0.08204600 \\ \mathrm{C} & 0.91631400 & -1.27331500 & -0.28423700 \\ \mathrm{H} & -1.05319500 & 2.16799500 & 0.03578600 \\ \mathrm{H} & -0.37602100 & -1.37884500 & 1.46336400 \\ \mathrm{H} & -1.05318300 & -2.16800300 & 0.03578500\end{array}$




$\begin{array}{lrrr}\mathrm{H} & 0.79977000 & 1.33310800 & -1.37504100 \\ \mathrm{H} & 1.47620300 & 2.16510300 & 0.02727500 \\ \mathrm{H} & 2.66953300 & 0.00000700 & -0.42382000 \\ \mathrm{H} & 1.90510100 & 0.00000500 & 1.16287200 \\ \mathrm{H} & 0.79977800 & -1.33310400 & -1.37504200 \\ \mathrm{H} & 1.47621600 & -2.16509500 & 0.02727400 \\ \mathrm{H} & -0.37603000 & 1.37884100 & 1.46336500 \\ \mathrm{C} & -1.21921700 & -0.00000500 & 0.06378800 \\ \mathrm{C} & -2.39957900 & -0.00000200 & -0.49249700 \\ & & \mathrm{H}_{3} \mathrm{~B}-\mathrm{NMe}_{3} & \\ & \mathbf{2 a} & \end{array}$

SCF energy[M062X-D3/6-311+G(2df,2p)/SMD(DCM)]: -201.11326299 a.u.

Thermal correction to Enthalpy at $273 \mathrm{~K}: 0.162229$ a.u.

Thermal correction to Gibbs free energy at $273 \mathrm{~K}: 0.130292$ a.u.

01

$\begin{array}{lrrr}\text { B } & 0.00000000 & 0.00000000 & 1.66594700 \\ \mathrm{H} & 0.00000000 & -1.17047900 & 1.98868900 \\ \mathrm{H} & -1.01366500 & 0.58524000 & 1.98868900 \\ \mathrm{H} & 1.01366500 & 0.58524000 & 1.98868900 \\ \mathrm{~N} & 0.00000000 & 0.00000000 & 0.00596600 \\ \mathrm{C} & 0.00000000 & 1.40190300 & -0.49387100 \\ \mathrm{H} & -0.88762600 & 1.90957000 & -0.11250000 \\ \mathrm{H} & 0.88762600 & 1.90957000 & -0.11250000 \\ \mathrm{H} & 0.00000000 & 1.41888200 & -1.59096000 \\ \mathrm{C} & -1.21408400 & -0.70095100 & -0.49387100 \\ \mathrm{H} & -1.20992300 & -1.72349200 & -0.11250000 \\ \mathrm{H} & -2.09754900 & -0.18607800 & -0.11250000 \\ \mathrm{H} & -1.22878800 & -0.70944100 & -1.59096000 \\ \mathrm{C} & 1.21408400 & -0.70095100 & -0.49387100\end{array}$




$\begin{array}{llll}\mathrm{H} & 2.09754900 & -0.18607800 & -0.11250000 \\ \mathrm{H} & 1.20992300 & -1.72349200 & -0.11250000 \\ \mathrm{H} & 1.22878800 & -0.70944100 & -1.59096000\end{array}$

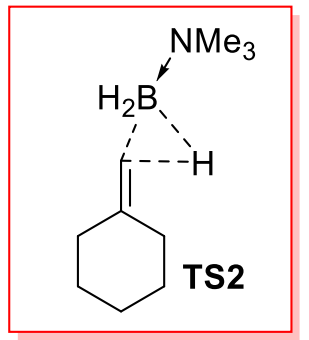

SCF energy[M062X-D3/6-311+G(2df,2p)/SMD(DCM)]: -473.73660809 a.u.

Thermal correction to Enthalpy at 273 K: 0.321209 a.u.

Thermal correction to Gibbs free energy at $273 \mathrm{~K}: 0.273793$ a.u.

01

$\mathrm{C}$

$\mathrm{C}$

$\mathrm{C}$

$\mathrm{C}$

$\mathrm{C}$

$\mathrm{H}$

$\mathrm{H}$

$\mathrm{H}$

$\mathrm{H}$

$\mathrm{H}$

H

H

H

H

H

C

C

B

$\begin{array}{rrr}-2.20989200 & -1.37514700 & -0.46560400 \\ -1.79965400 & 1.10539500 & -0.79324900 \\ -2.79925100 & 1.41979000 & 0.34097600 \\ -3.85886900 & 0.31602400 & 0.48025400 \\ -3.20868000 & -1.06299100 & 0.66831500 \\ -1.02704600 & 1.88138300 & -0.84220400 \\ -2.77381800 & -1.47696600 & -1.40850600 \\ -1.70448200 & -2.33086500 & -0.28856800 \\ -2.24597700 & 1.51449700 & 1.28664100 \\ -3.28077500 & 2.39000000 & 0.15565100 \\ -4.53071600 & 0.53973100 & 1.32031600 \\ -4.48522600 & 0.30008600 & -0.42566200 \\ -2.67517400 & -1.08575600 & 1.63010300 \\ -3.97966700 & -1.84489300 & 0.71399000 \\ -2.34560800 & 1.13608100 & -1.75191700 \\ -1.17568000 & -0.26838800 & -0.62107100 \\ 0.12514600 & -0.63501500 & -0.58126300 \\ 1.51530600 & 0.97161100 & -0.15962100\end{array}$




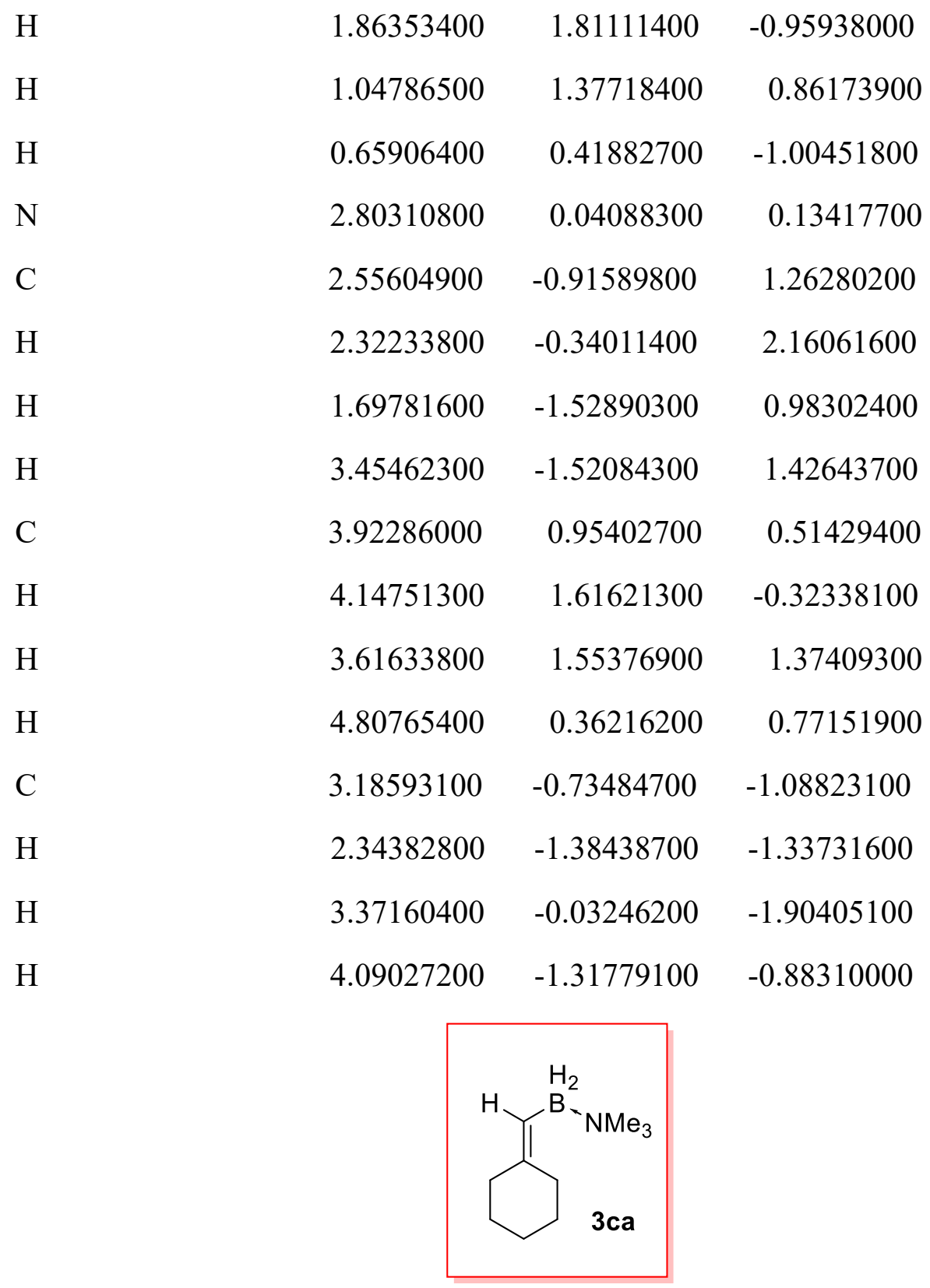

SCF energy[M062X-D3/6-311+G(2df,2p)/SMD(DCM)]: -473.85238860 a.u.

Thermal correction to Enthalpy at $273 \mathrm{~K}: 0.325569$ a.u.

Thermal correction to Gibbs free energy at $273 \mathrm{~K}: 0.277831$ a.u.

01

$\begin{array}{lrrr}\mathrm{C} & 1.41764900 & 1.08136200 & -0.38322300 \\ \mathrm{C} & 2.09036600 & -1.35258700 & -0.41091200 \\ \mathrm{C} & 3.43220300 & -1.00132600 & 0.26700900 \\ \mathrm{C} & 3.86277900 & 0.43676100 & -0.05932200 \\ \mathrm{C} & 2.75816400 & 1.44466400 & 0.29283500 \\ & & \mathrm{~S} 65 & \end{array}$




\begin{tabular}{|c|c|c|c|}
\hline $\mathrm{H}$ & 1.77313700 & -2.36439600 & -0.12844400 \\
\hline $\mathrm{H}$ & 1.54749100 & 1.18664300 & -1.47490300 \\
\hline $\mathrm{H}$ & 0.63867600 & 1.78525000 & -0.07962600 \\
\hline $\mathrm{H}$ & 3.32012800 & -1.10975500 & 1.35566100 \\
\hline $\mathrm{H}$ & 4.20866900 & -1.71325600 & -0.04556600 \\
\hline $\mathrm{H}$ & 4.79040800 & 0.68625500 & 0.47390800 \\
\hline $\mathrm{H}$ & 4.09093400 & 0.51068200 & -1.13449600 \\
\hline $\mathrm{H}$ & 2.61200200 & 1.45983900 & 1.38255300 \\
\hline $\mathrm{H}$ & 3.06101200 & 2.45933100 & -0.00067800 \\
\hline $\mathrm{H}$ & 2.25269800 & -1.36009100 & -1.50290700 \\
\hline $\mathrm{C}$ & 1.00629500 & -0.34451600 & -0.07064900 \\
\hline $\mathrm{C}$ & -0.16828500 & -0.70315100 & 0.49468500 \\
\hline $\mathrm{B}$ & -1.37815600 & 0.20536200 & 1.01823600 \\
\hline $\mathrm{H}$ & -1.81229400 & -0.18002800 & 2.08928400 \\
\hline $\mathrm{H}$ & -1.18355100 & 1.40359700 & 1.01785100 \\
\hline $\mathrm{H}$ & -0.25239400 & -1.78459000 & 0.67197900 \\
\hline $\mathrm{N}$ & -2.72750300 & 0.04961100 & 0.00111000 \\
\hline $\mathrm{C}$ & -2.41059500 & 0.56457000 & -1.35858200 \\
\hline $\mathrm{H}$ & -2.12492800 & 1.61502800 & -1.27771600 \\
\hline $\mathrm{H}$ & -1.56829800 & -0.00128800 & -1.75977400 \\
\hline $\mathrm{H}$ & -3.28174800 & 0.46443000 & -2.01808400 \\
\hline $\mathrm{C}$ & -3.84178500 & 0.84775600 & 0.57852600 \\
\hline $\mathrm{H}$ & -4.07097400 & 0.46361100 & 1.57398800 \\
\hline $\mathrm{H}$ & -3.52012100 & 1.88678300 & 0.66800500 \\
\hline $\mathrm{H}$ & -4.72973300 & 0.78337700 & -0.06282800 \\
\hline $\mathrm{C}$ & -3.15061500 & -1.37336900 & -0.09879600 \\
\hline $\mathrm{H}$ & -2.34434700 & -1.95365200 & -0.54989500 \\
\hline $\mathrm{H}$ & -3.34493600 & -1.75317800 & 0.90626000 \\
\hline $\mathrm{H}$ & -4.05559600 & -1.45966600 & -0.71295500 \\
\hline
\end{tabular}


9. NMR Spectra of New Compounds

(4-phenylcyclohexylidene)methyl trifluoromethanesulfonate (1a)
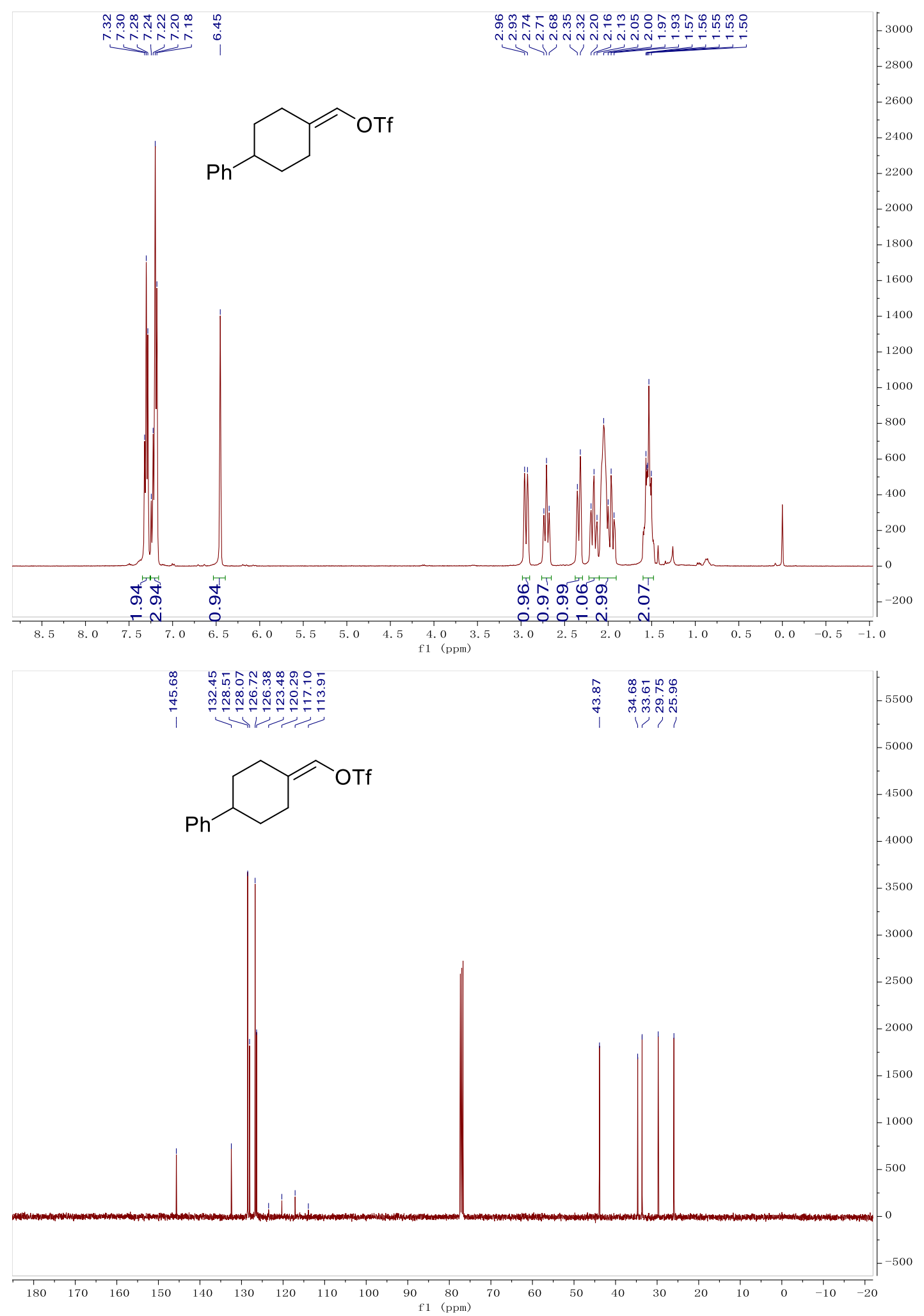
2-hexyloct-1-en-1-yl trifluoromethanesulfonate (1g)

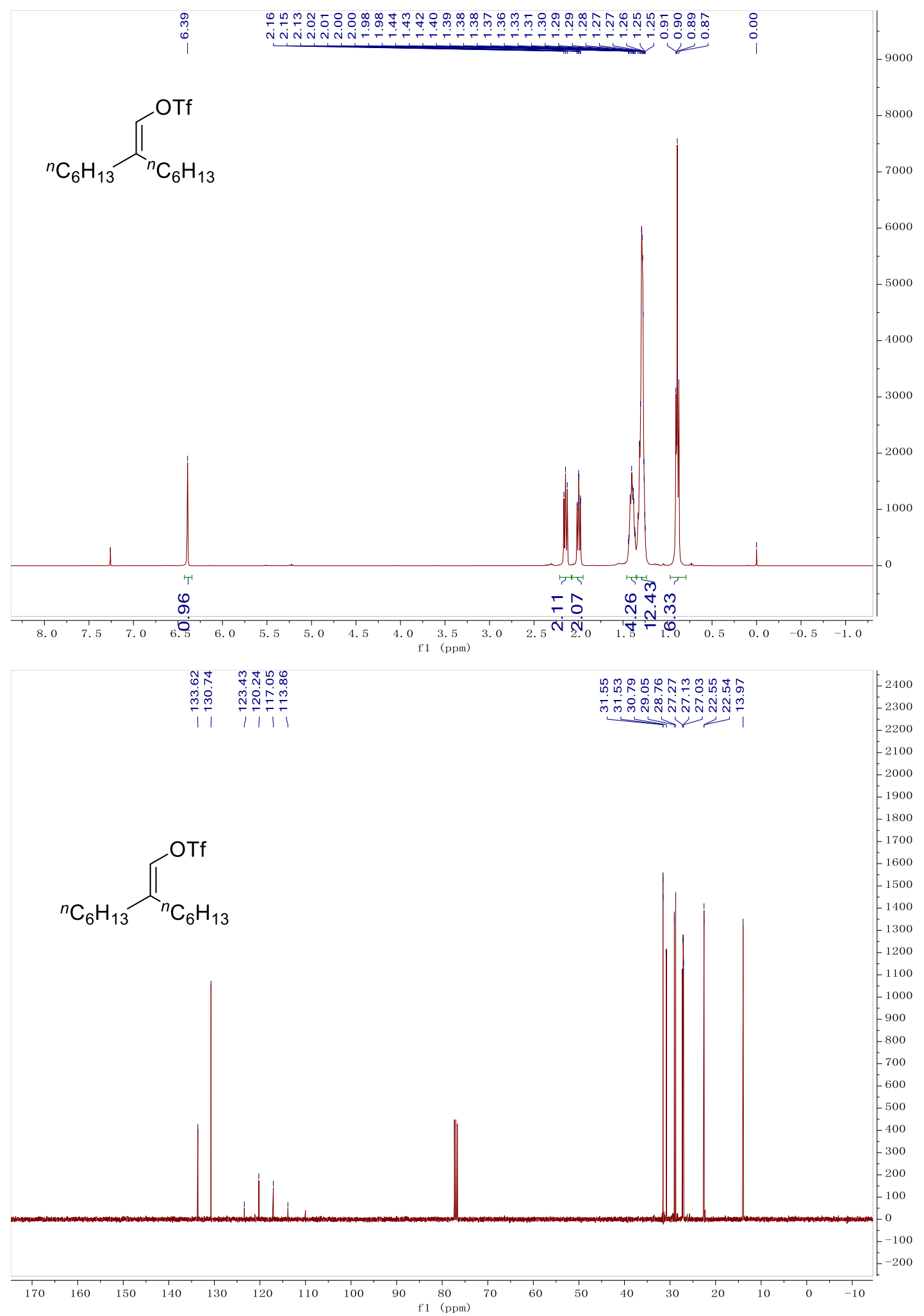




\section{3-(4-(tert-butyl)phenyl)-2-methylprop-1-en-1-yl trifluoromethanesulfonate (1h)}

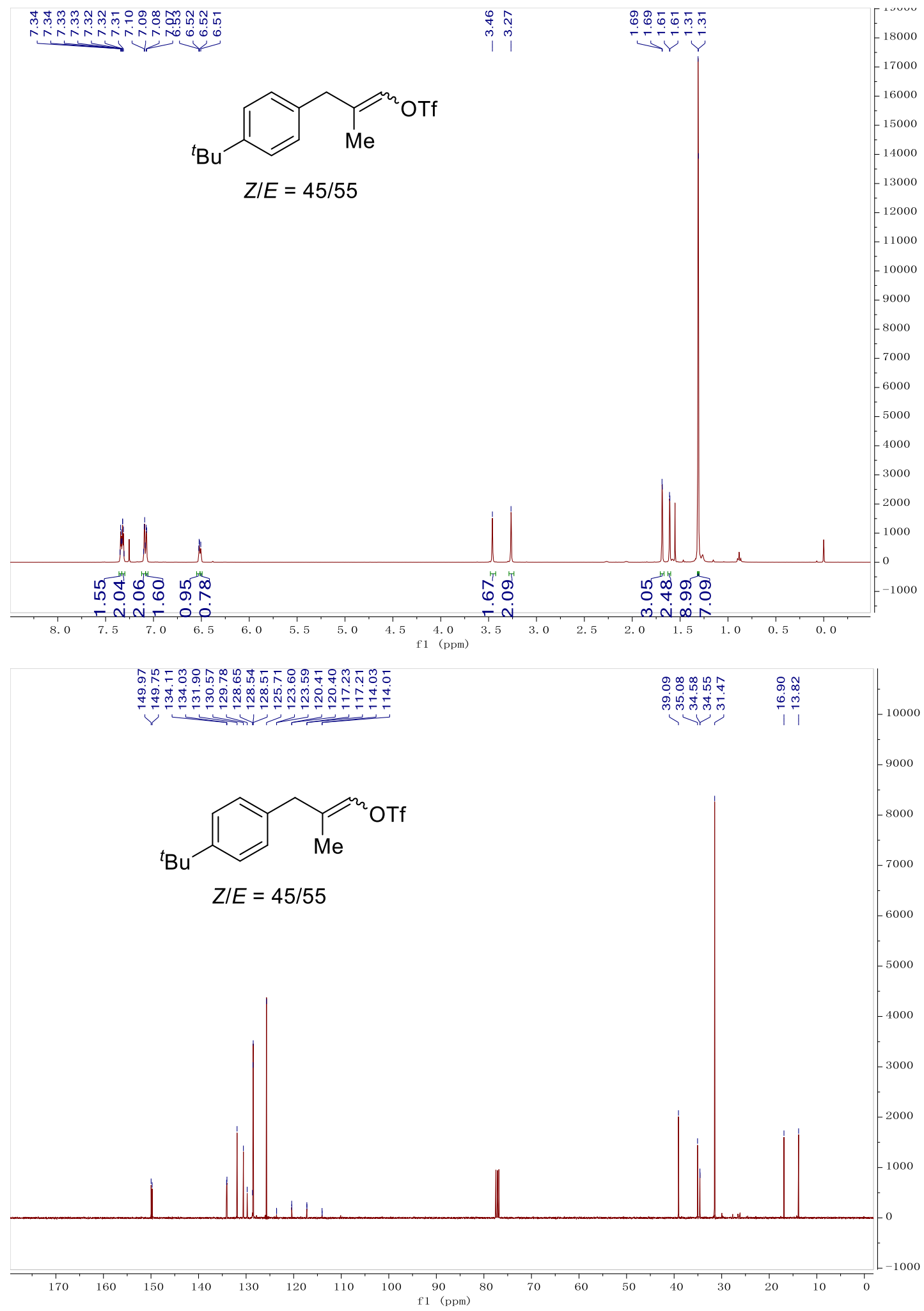


methyl (Z)-2-benzyl-3-(((trifluoromethyl)sulfonyl)oxy)acrylate $((Z)-S 3 a)$

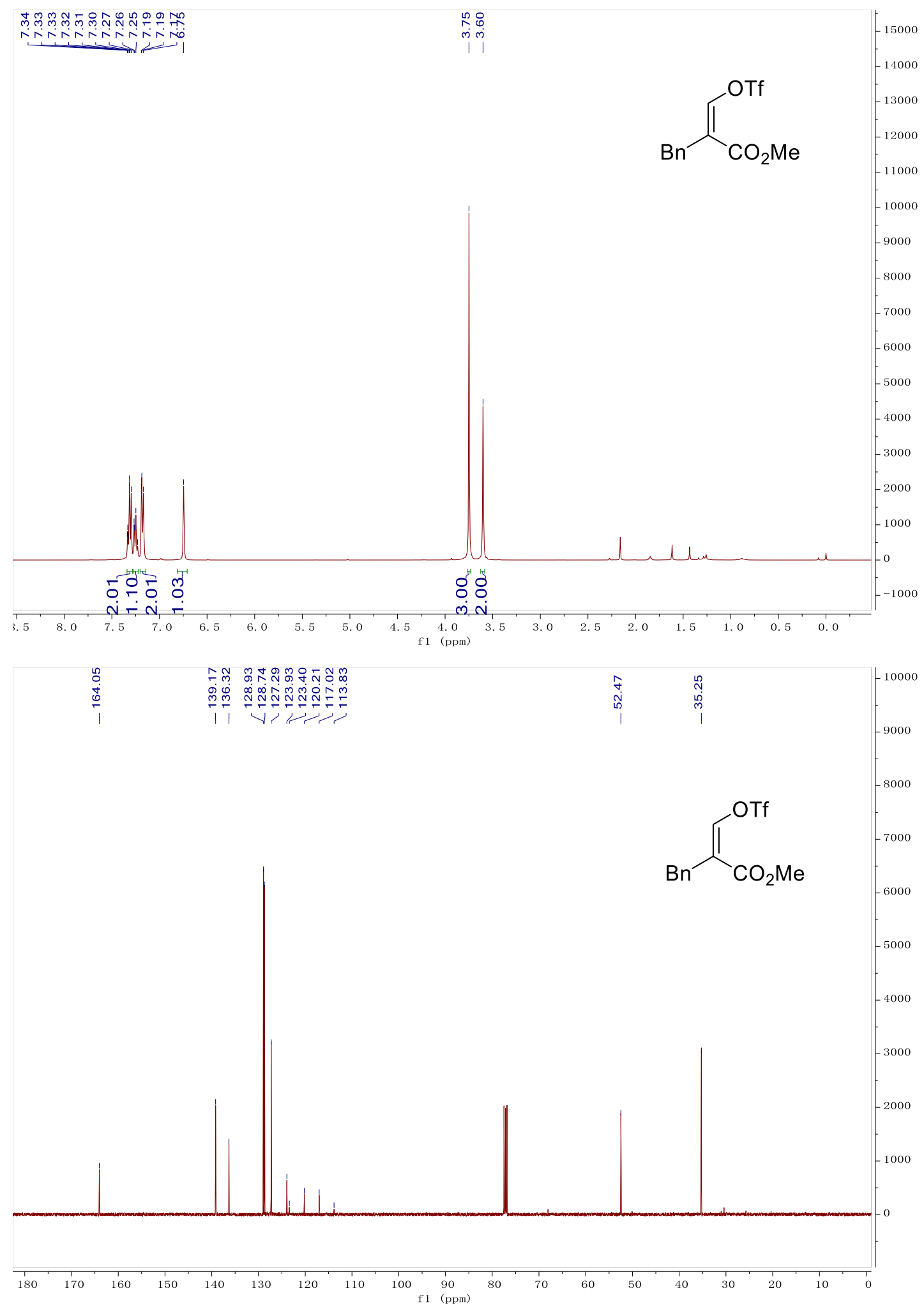



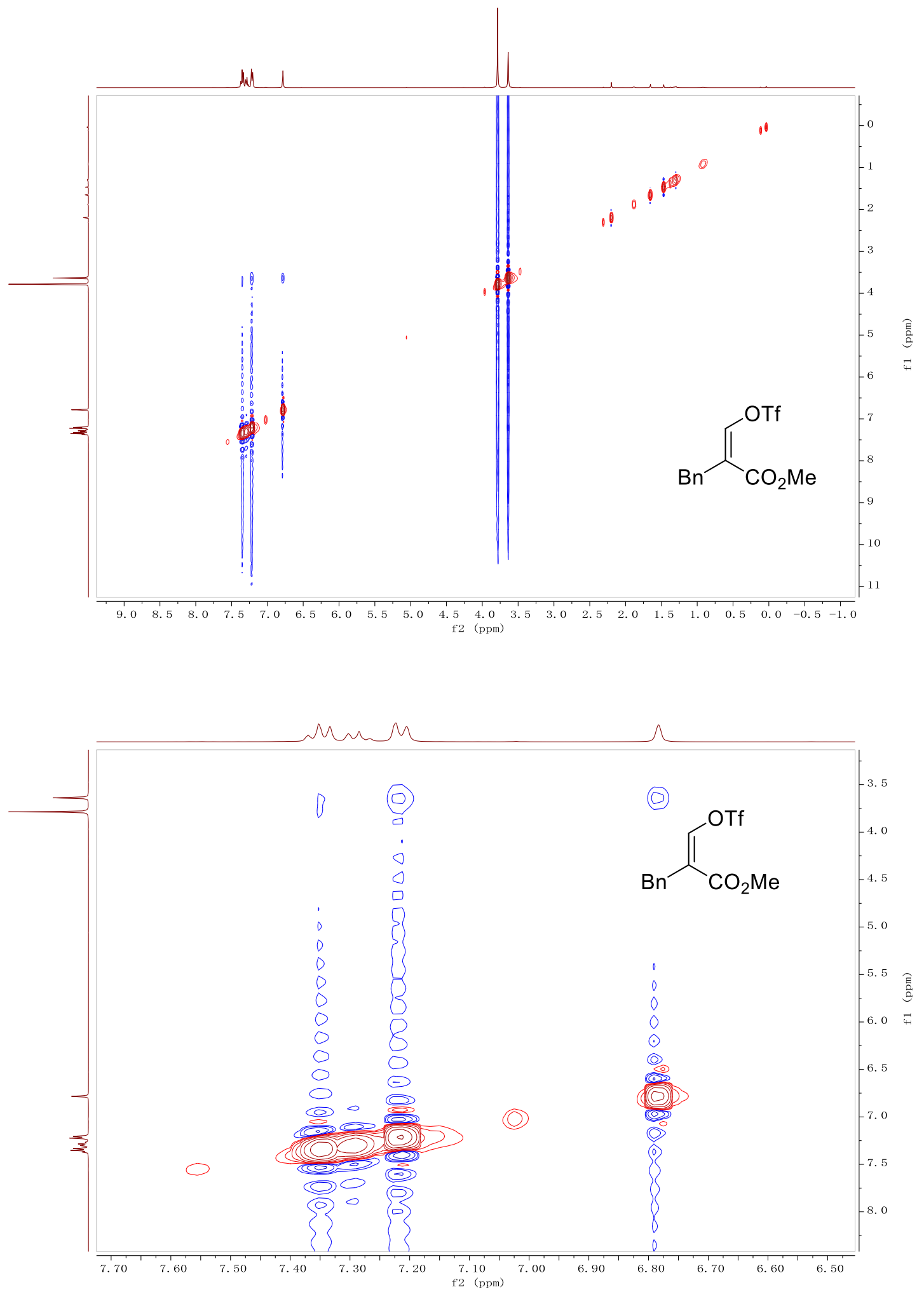
methyl $(E)$-2-benzyl-3-(((trifluoromethyl)sulfonyl)oxy)acrylate $((E)-S 3 a)$

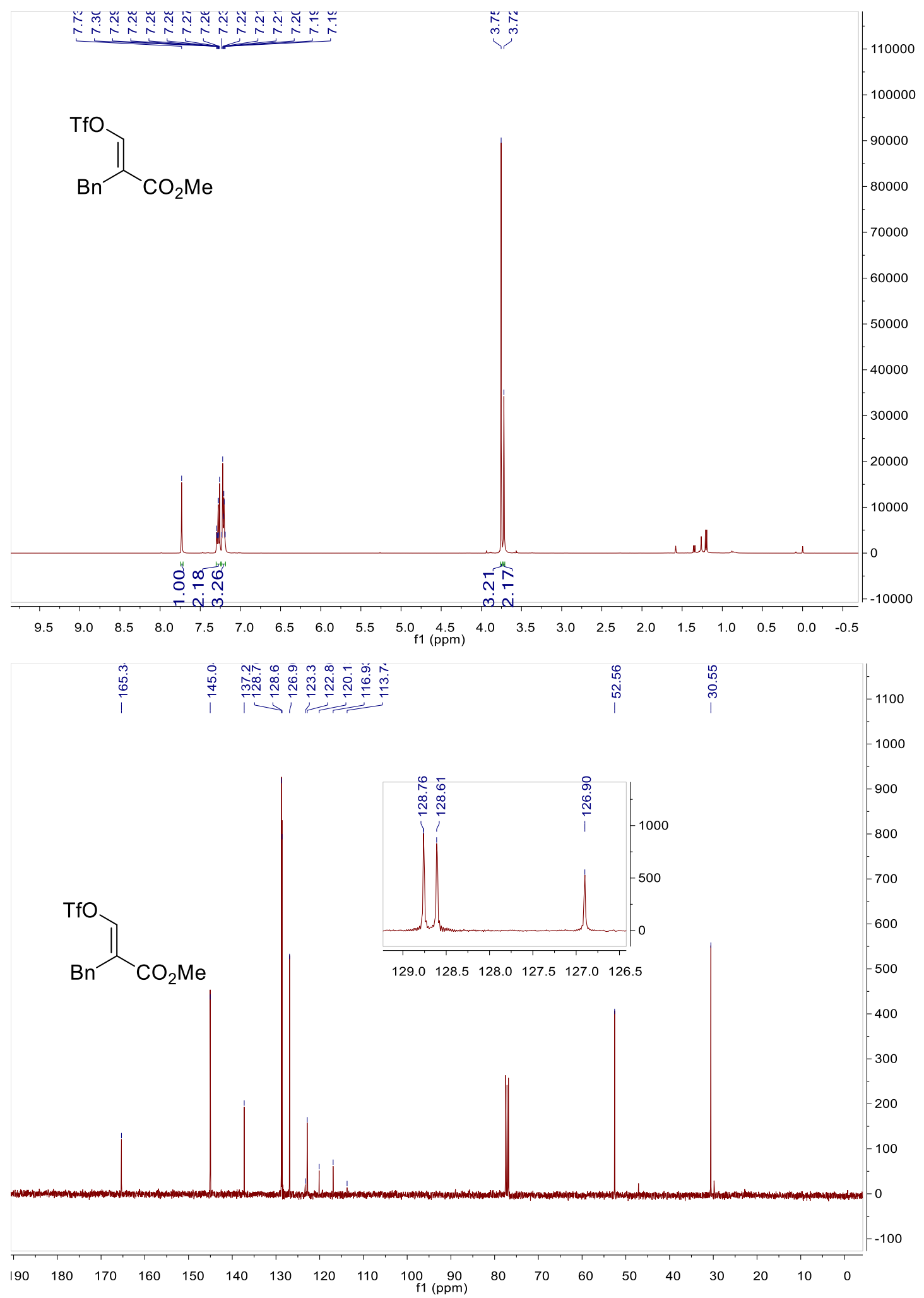



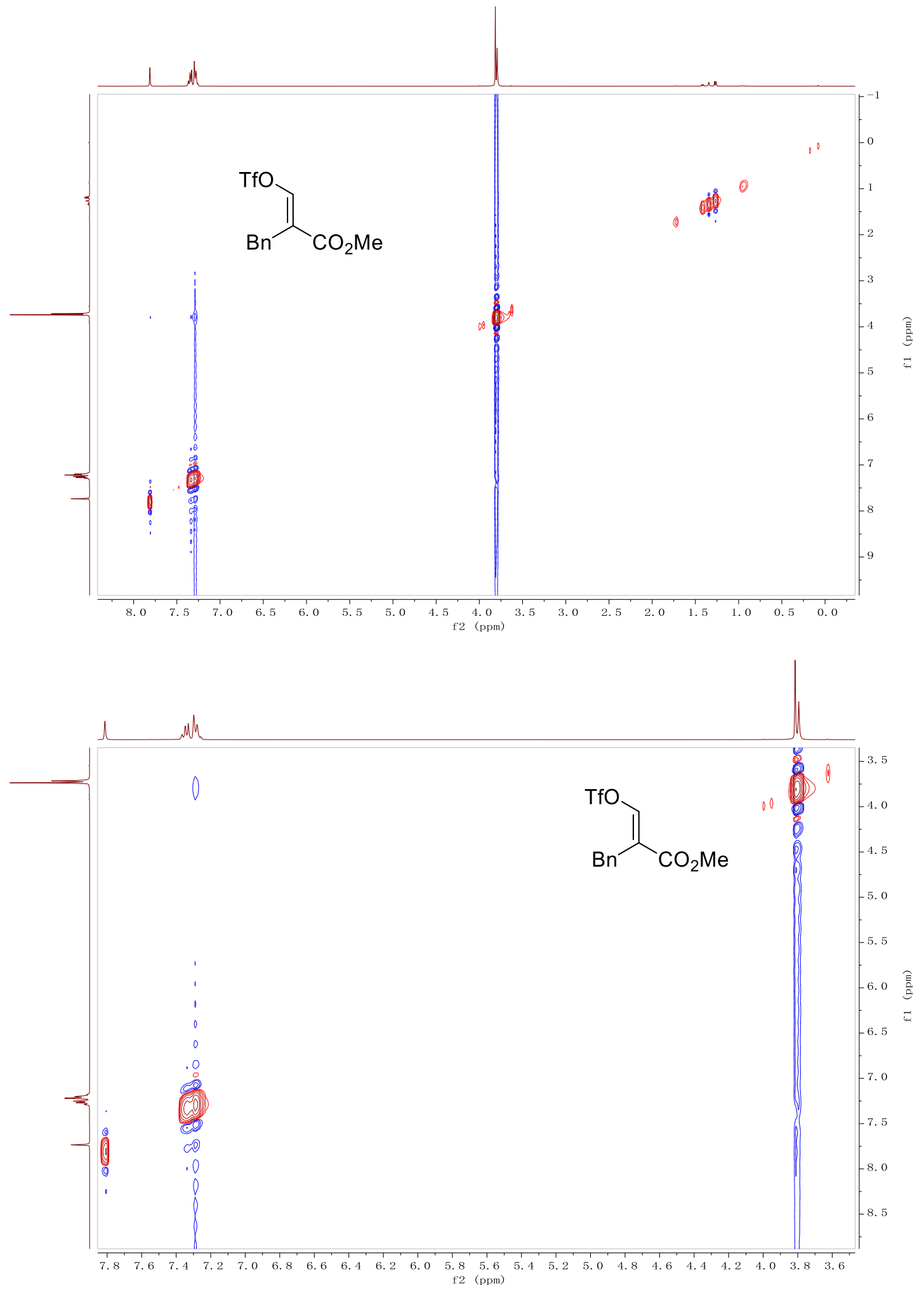
methyl $(E)-2-(4-c h l o r o b e n z y l)-3-(($ trifluoromethyl)sulfonyl)oxy)acrylate (E-S3n)

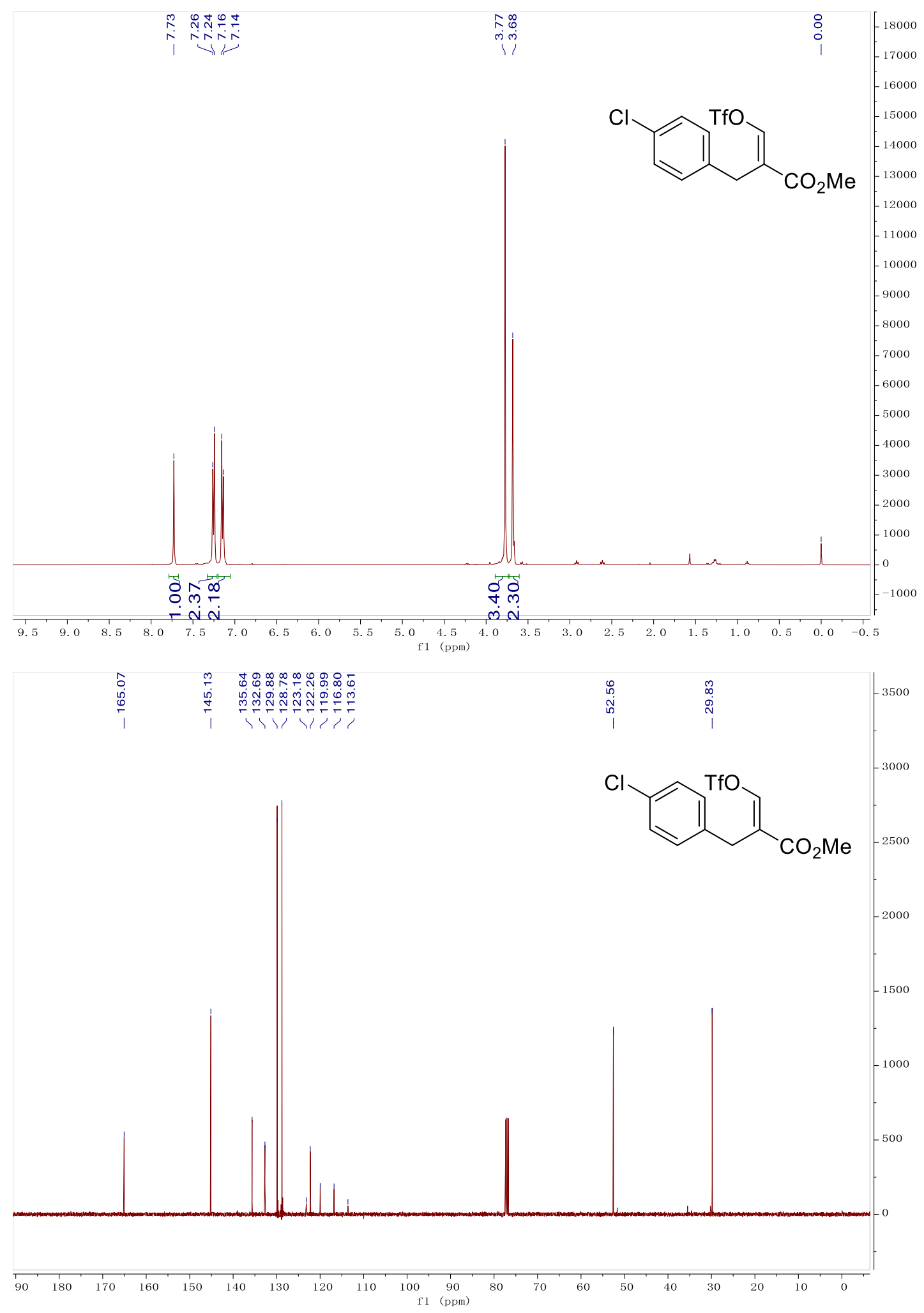



(S3o)
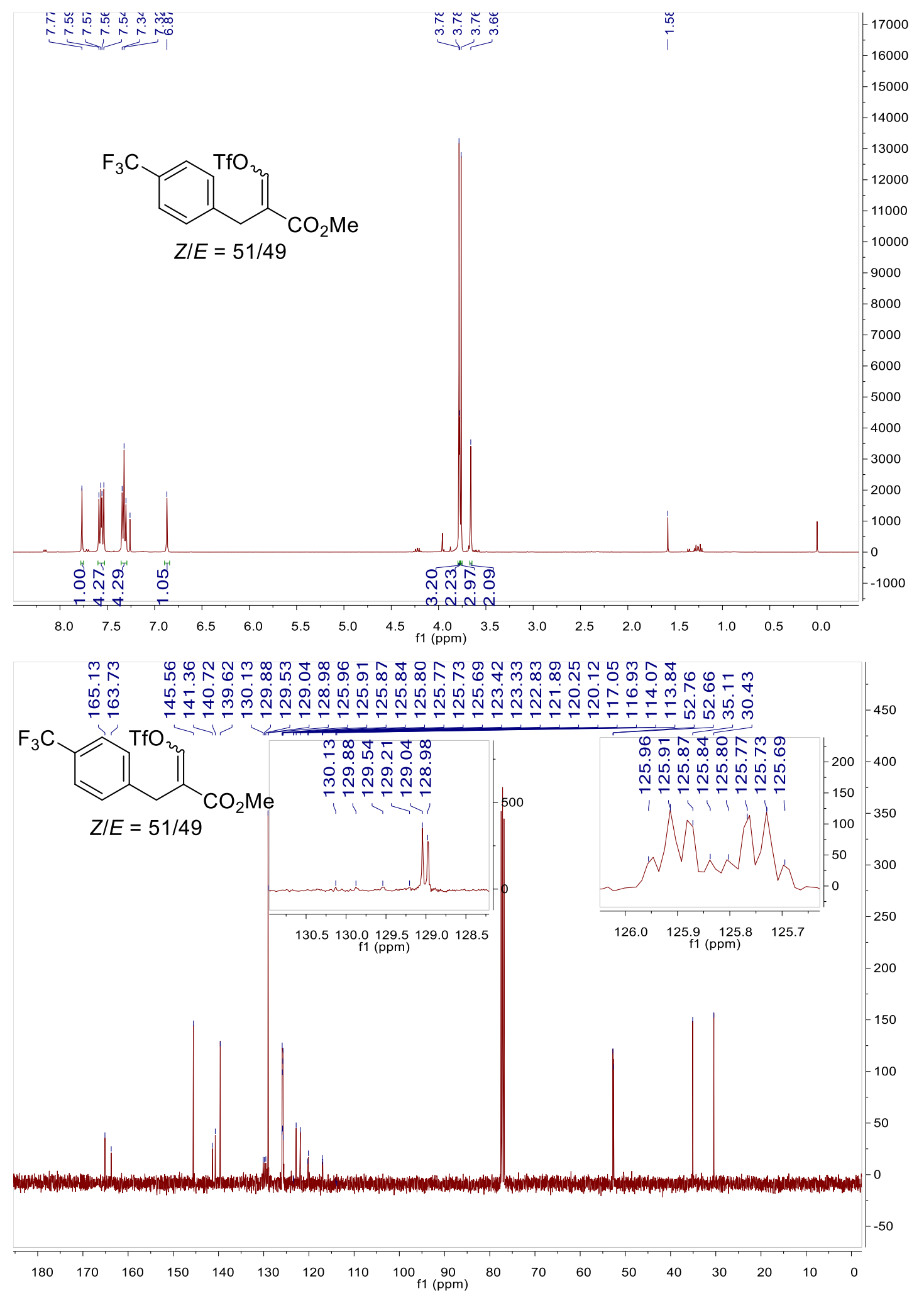
methyl 2-(4-methoxybenzyl)-3-(((trifluoromethyl)sulfonyl)oxy)acrylate (S3p)

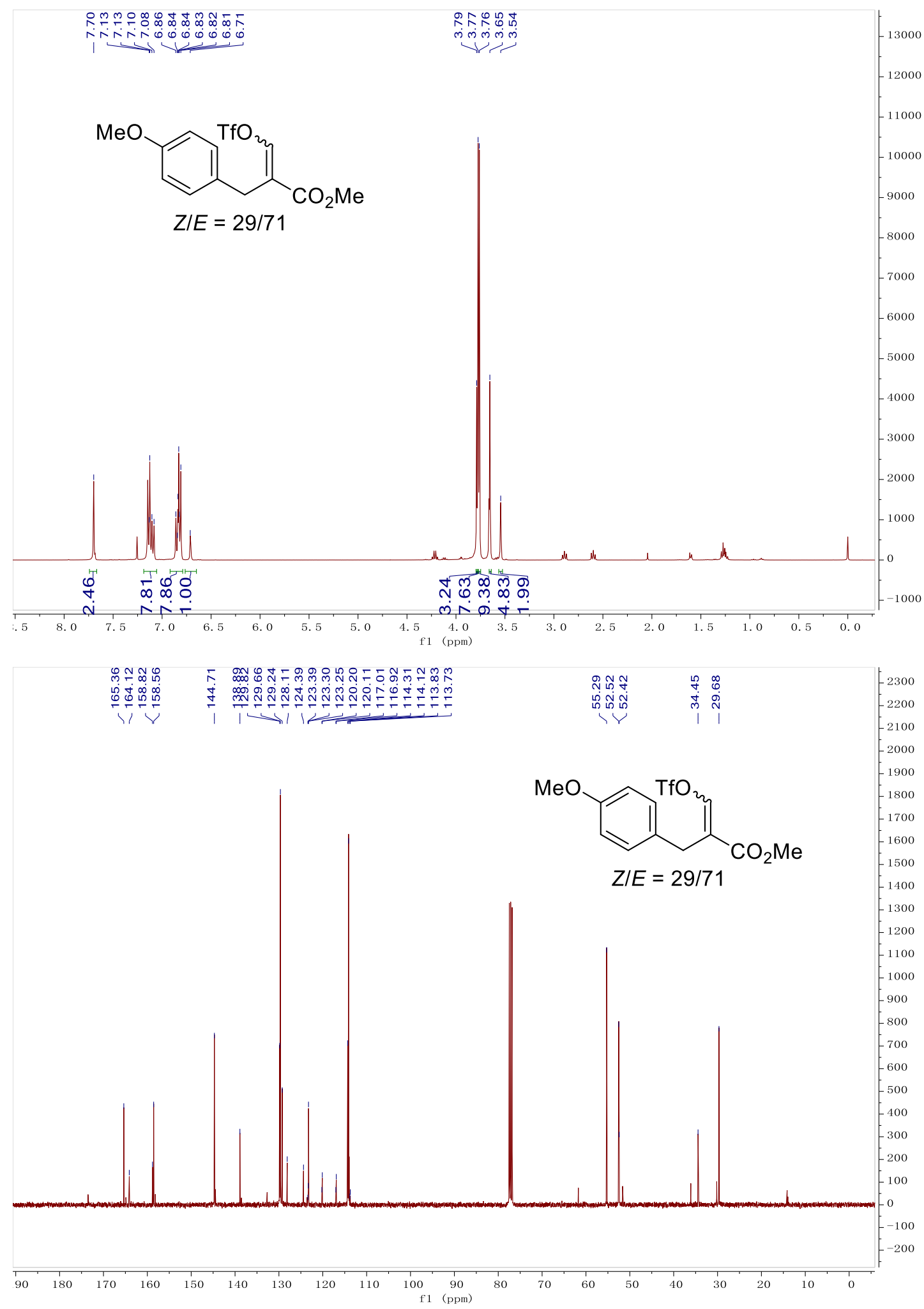


methyl 2-(4-fluorobenzyl)-3-(((trifluoromethyl)sulfonyl)oxy)acrylate (S3q)

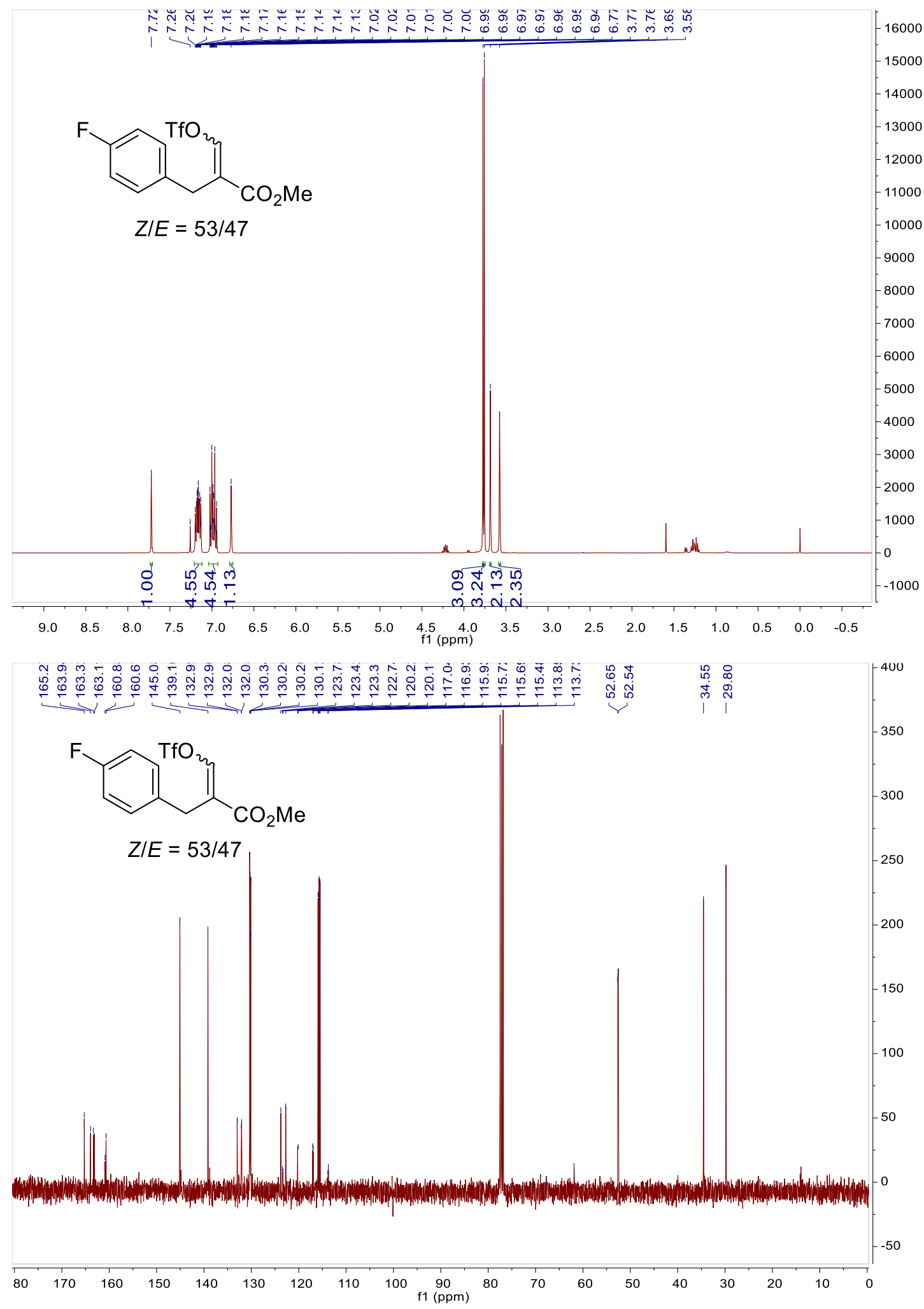


methyl 2-methyl-3-(((trifluoromethyl)sulfonyl)oxy)acrylate (S3r)

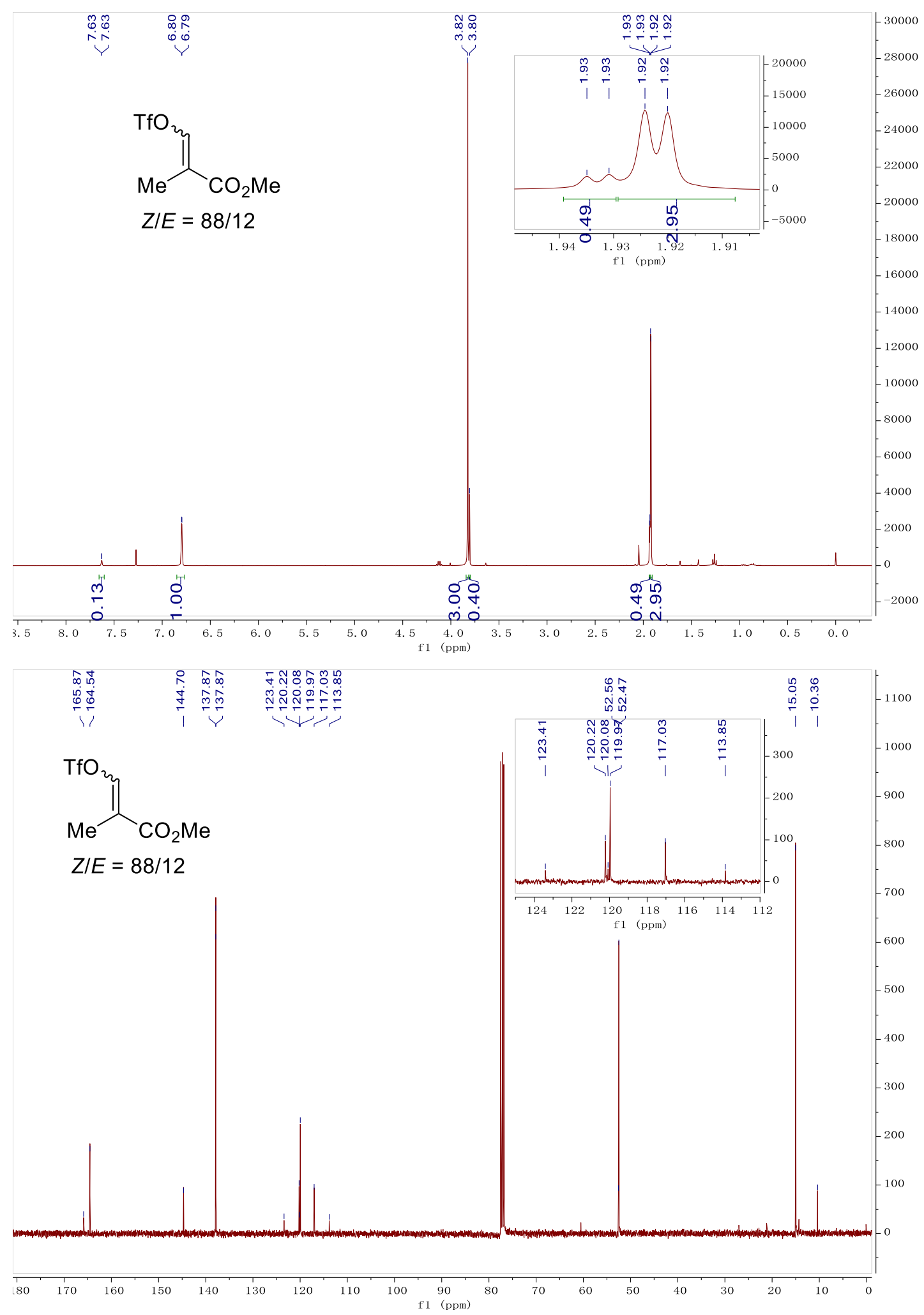


methyl 2-methyl-3-(((trifluoromethyl)sulfonyl)oxy)acrylate (S3s)

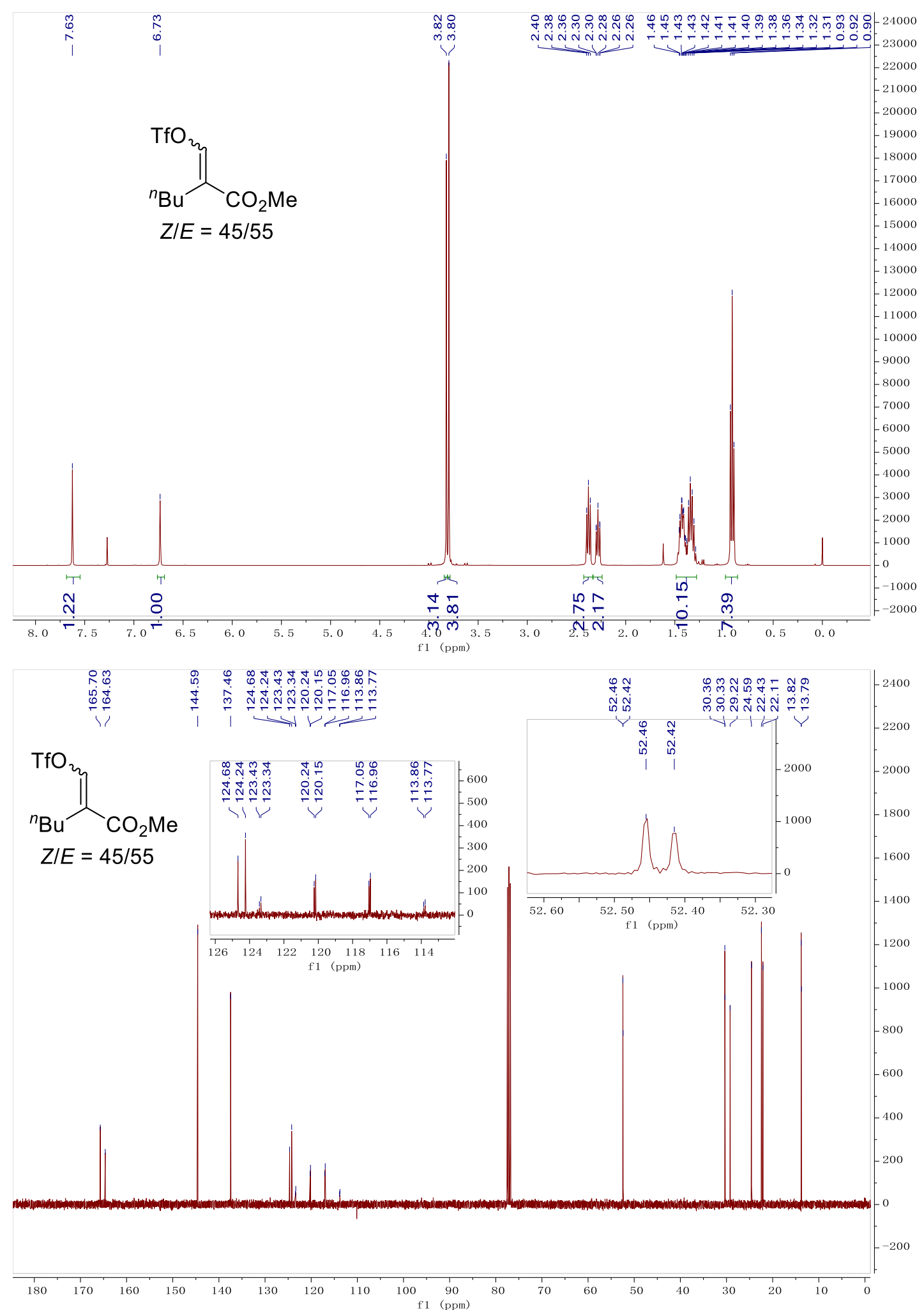


ethyl 3-methyl-2-((((trifluoromethyl)sulfonyl)oxy)methylene)butanoate (S3t)

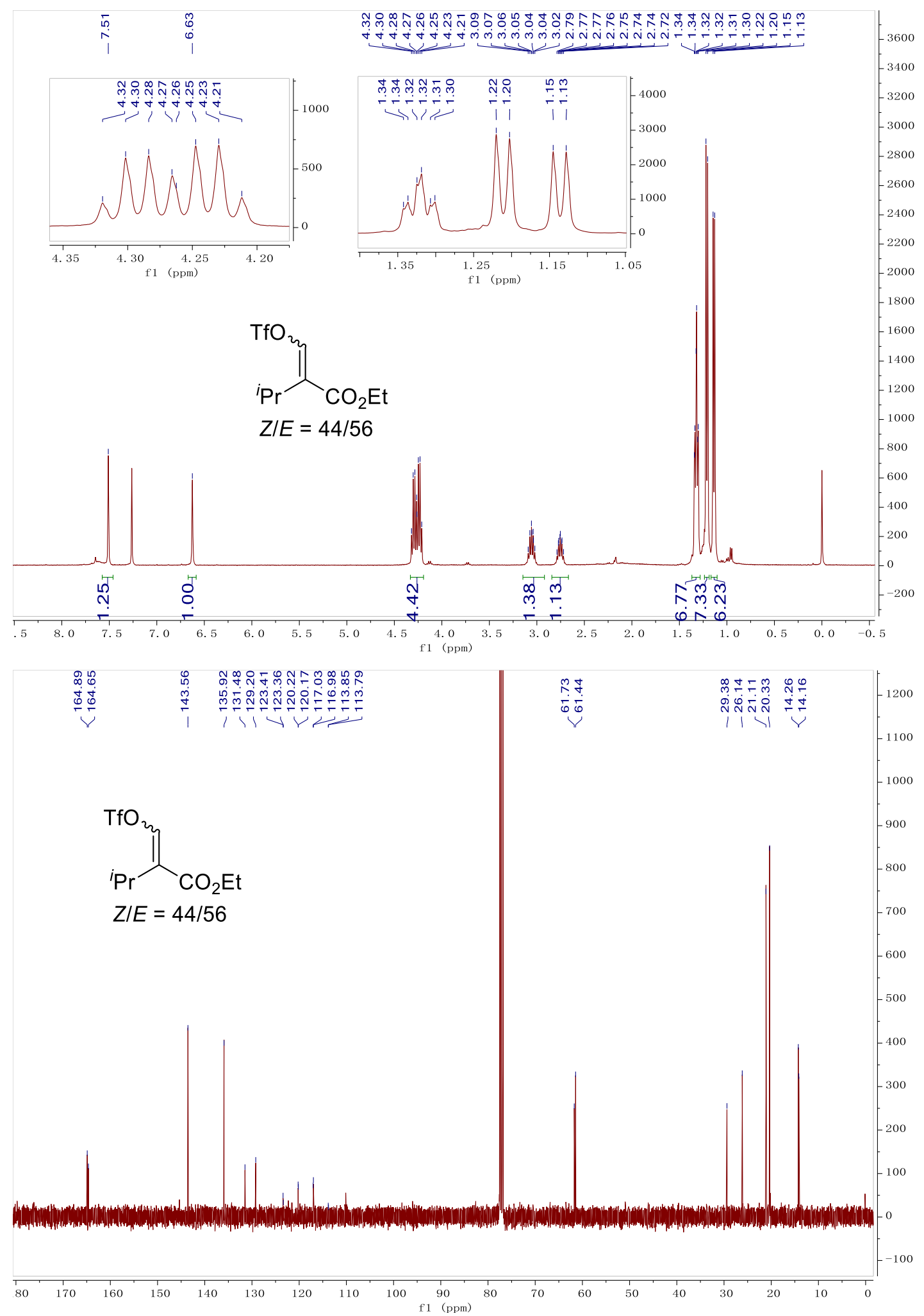


methyl 2-cyclohexyl-3-(((trifluoromethyl)sulfonyl)oxy)acrylate (S3u)

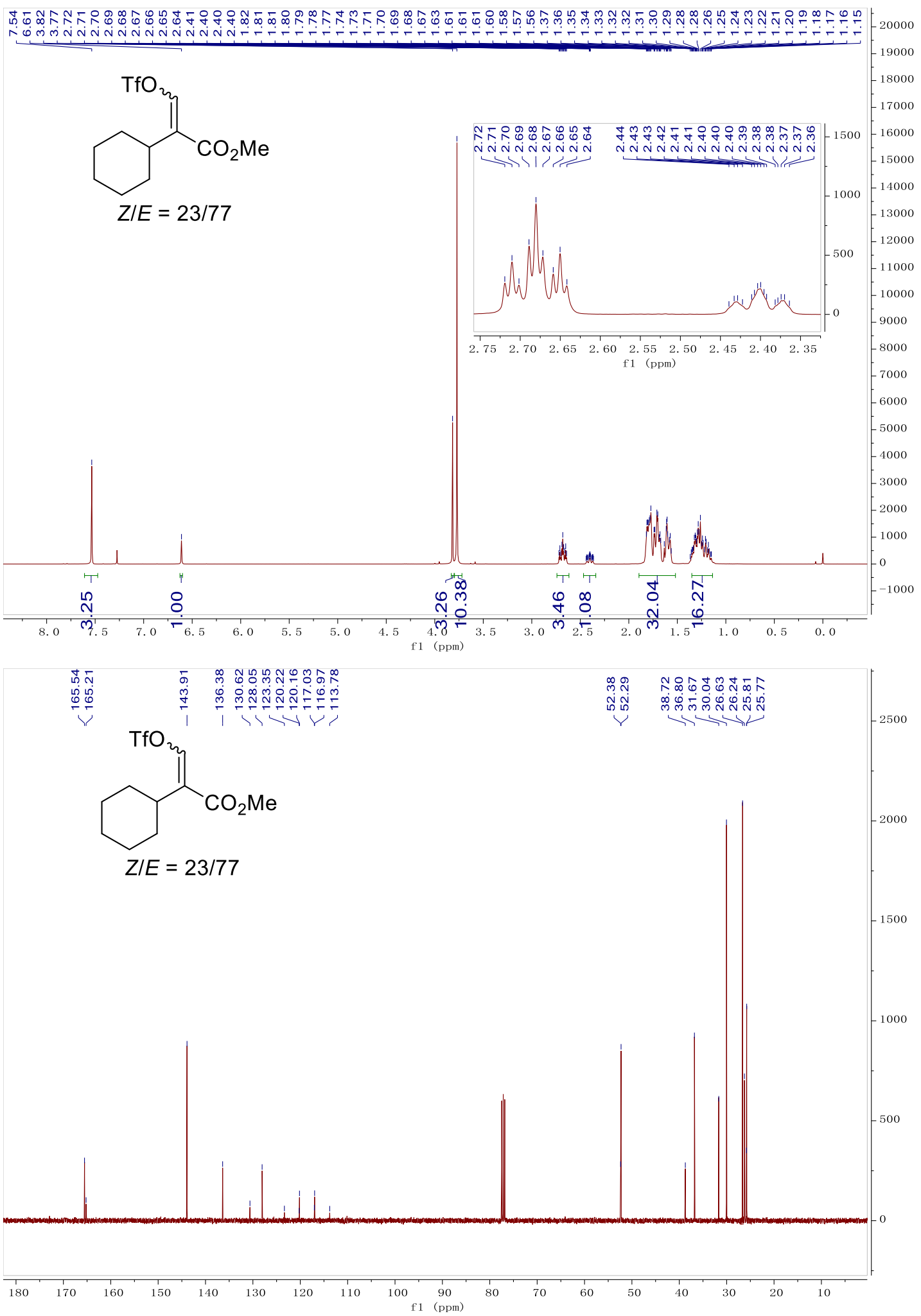


methyl (E)-2-phenyl-3-(((trifluoromethyl)sulfonyl)oxy)acrylate (S3v)

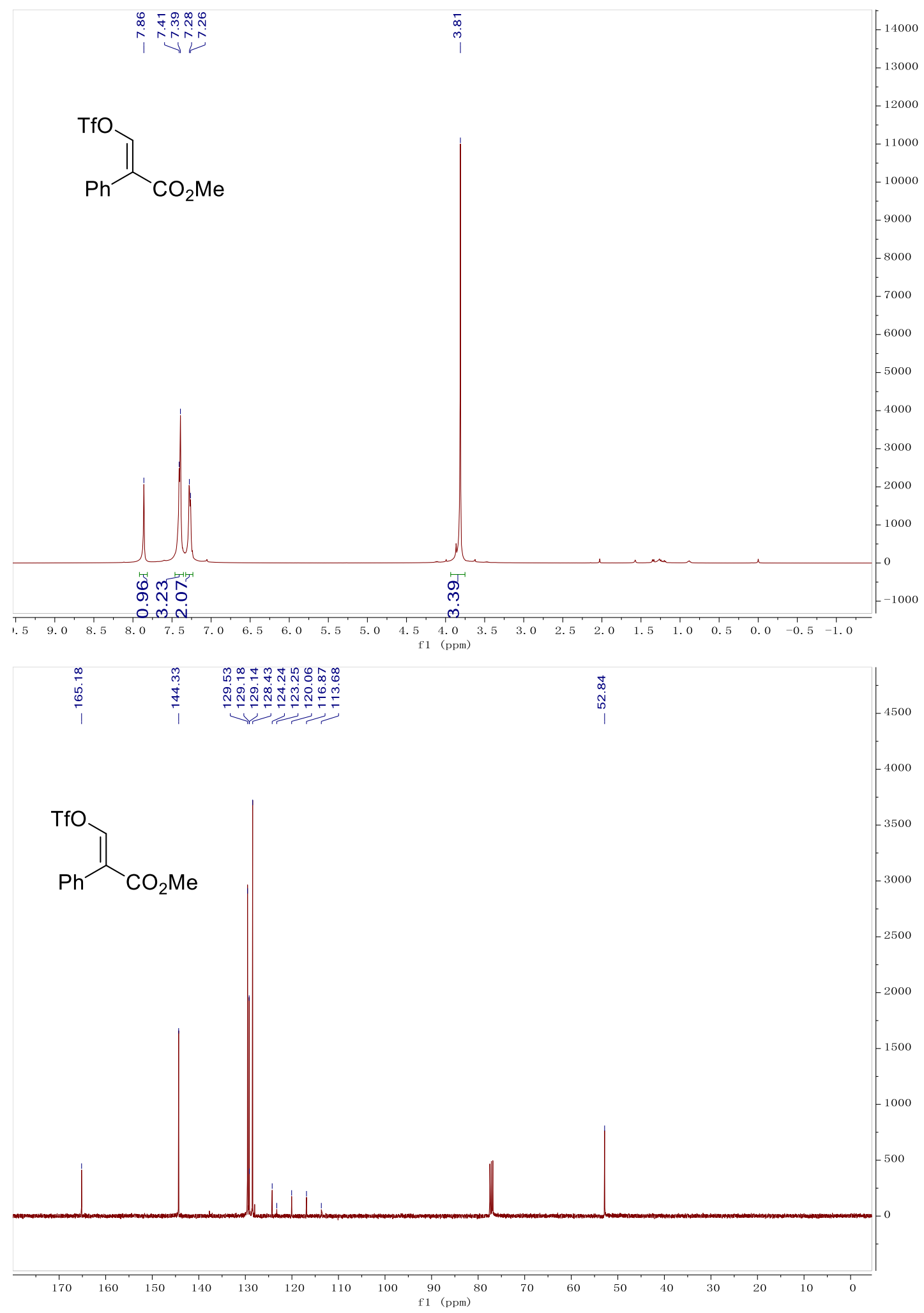


methyl (E)-2-(4-methoxyphenyl)-3-(((trifluoromethyl)sulfonyl)oxy)acrylate (S3w)

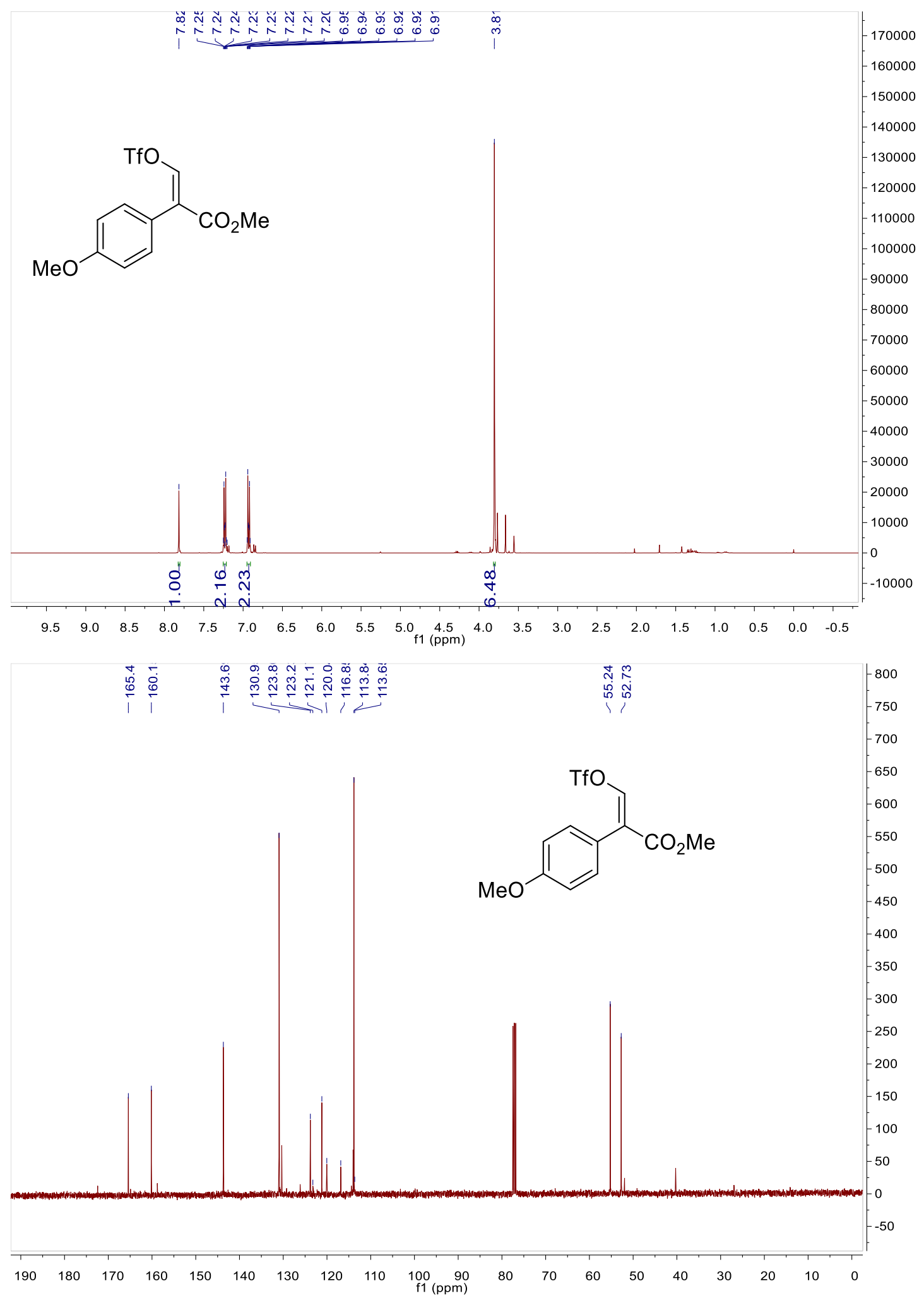


methyl (E)-2-(4-chlorophenyl)-3-(((trifluoromethyl)sulfonyl)oxy)acrylate (S3x)

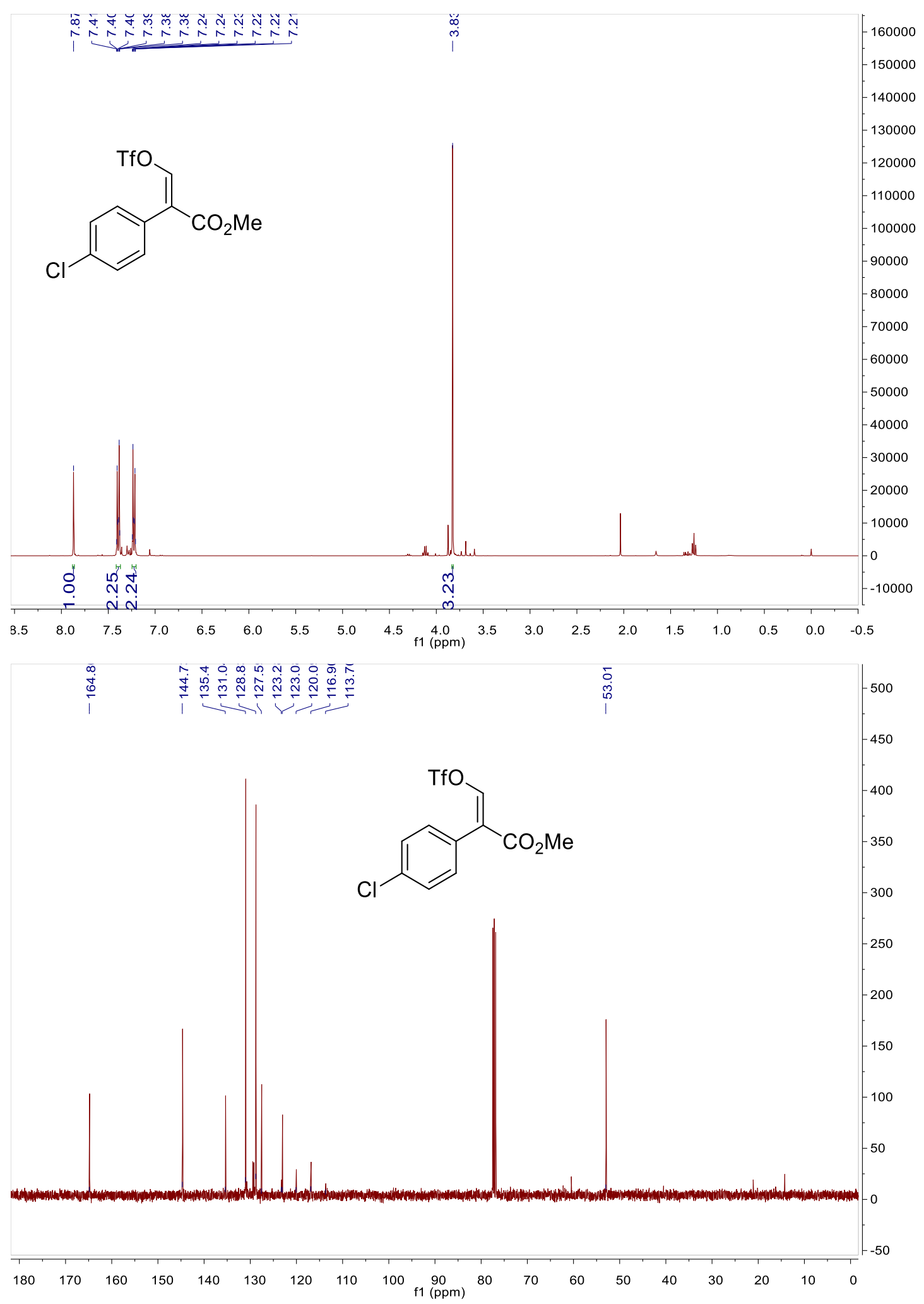



(S3y)

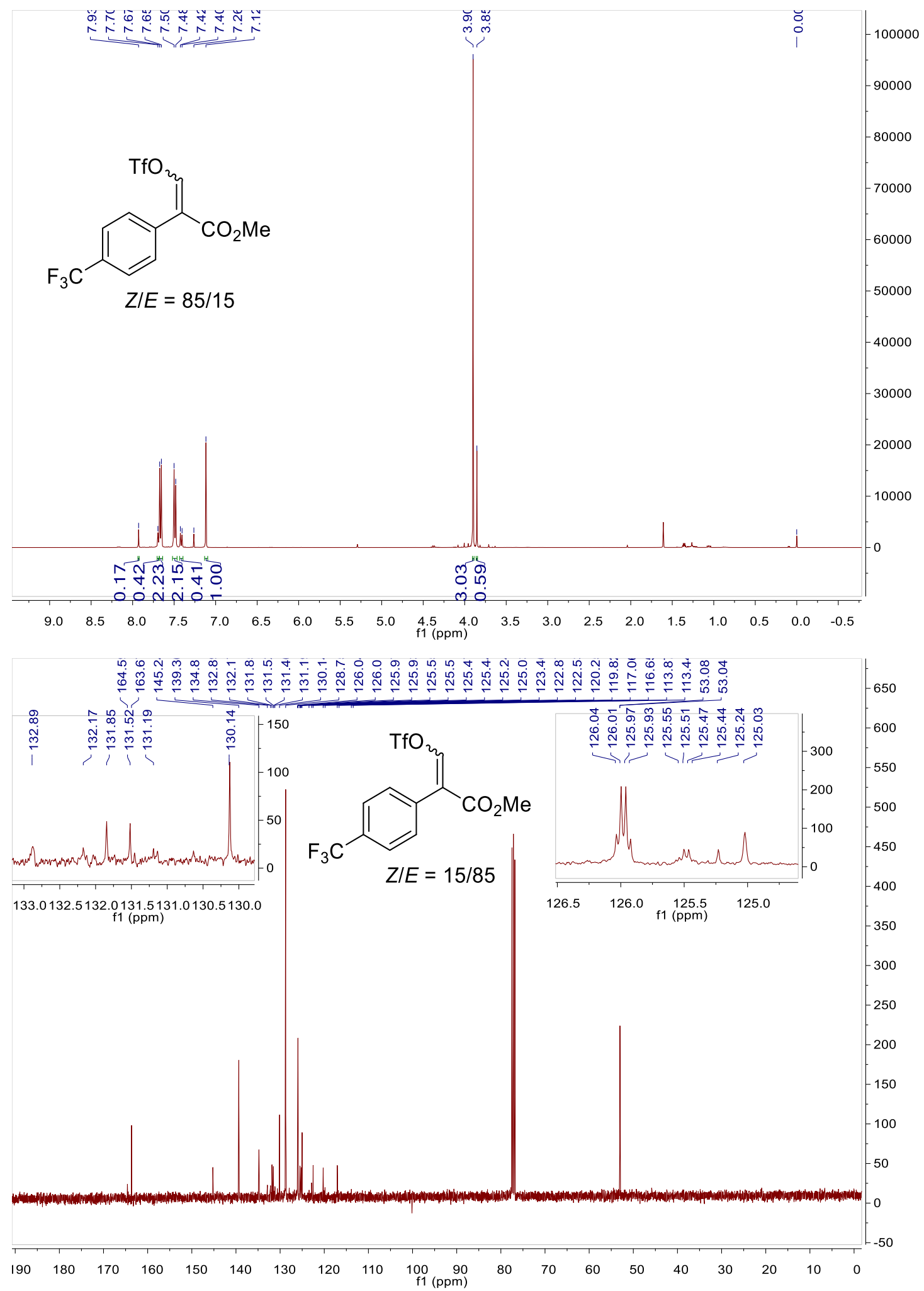



(4a)

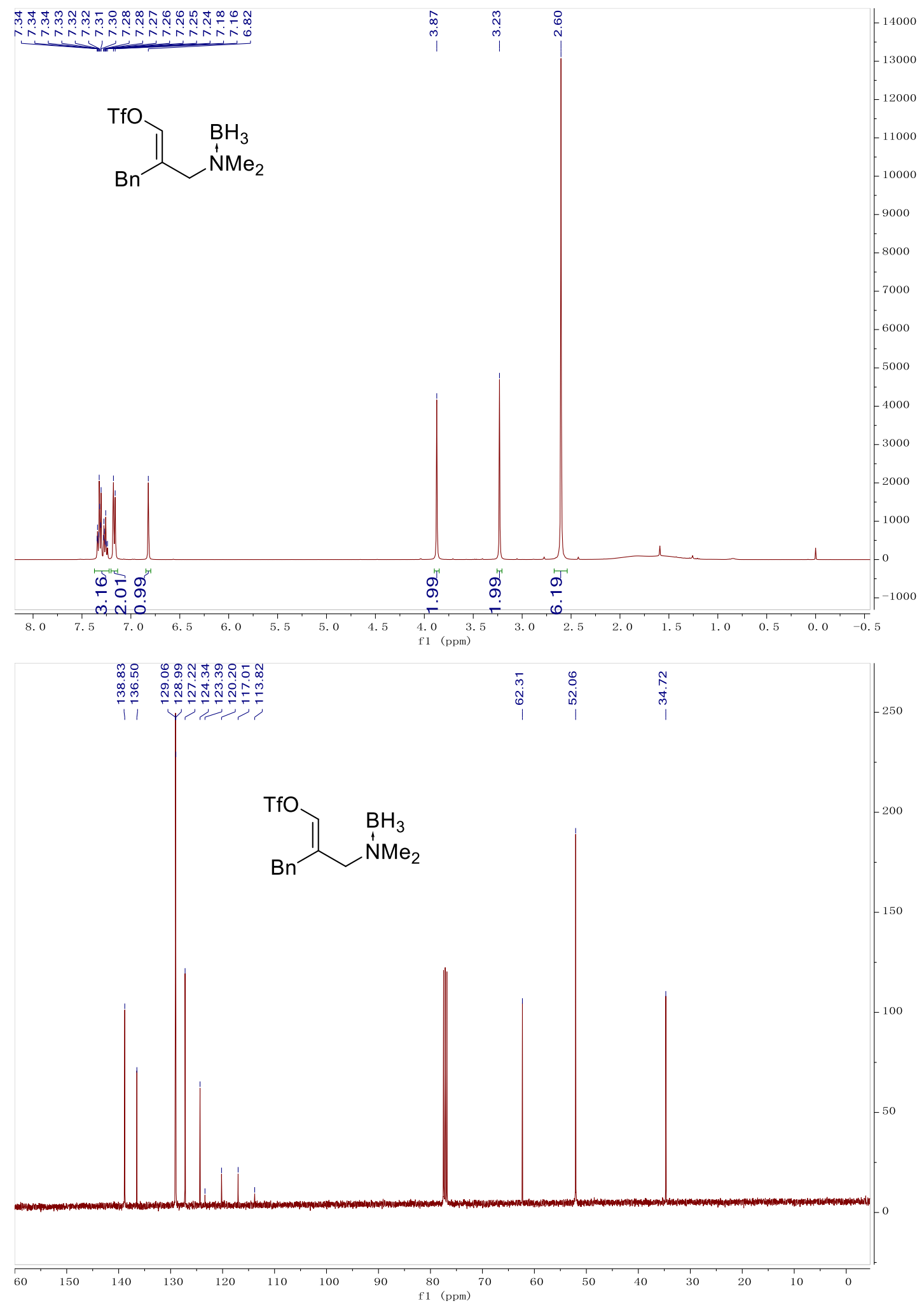



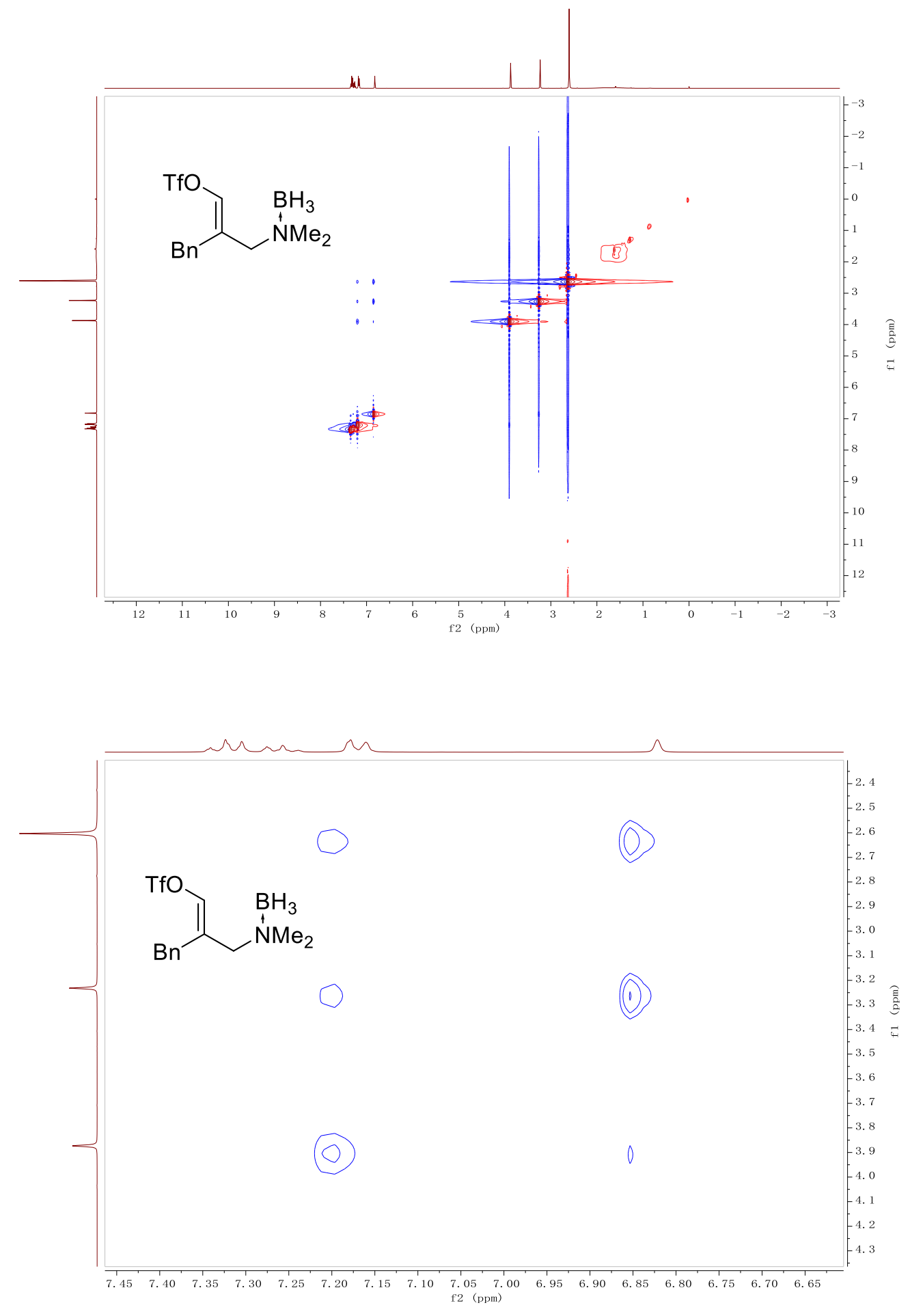


\section{(E)-2-benzyl-3-(butyl(methyl)aminoborane)prop-1-en-1-yl}

\section{trifluoromethanesulfonate $(4 b)$}

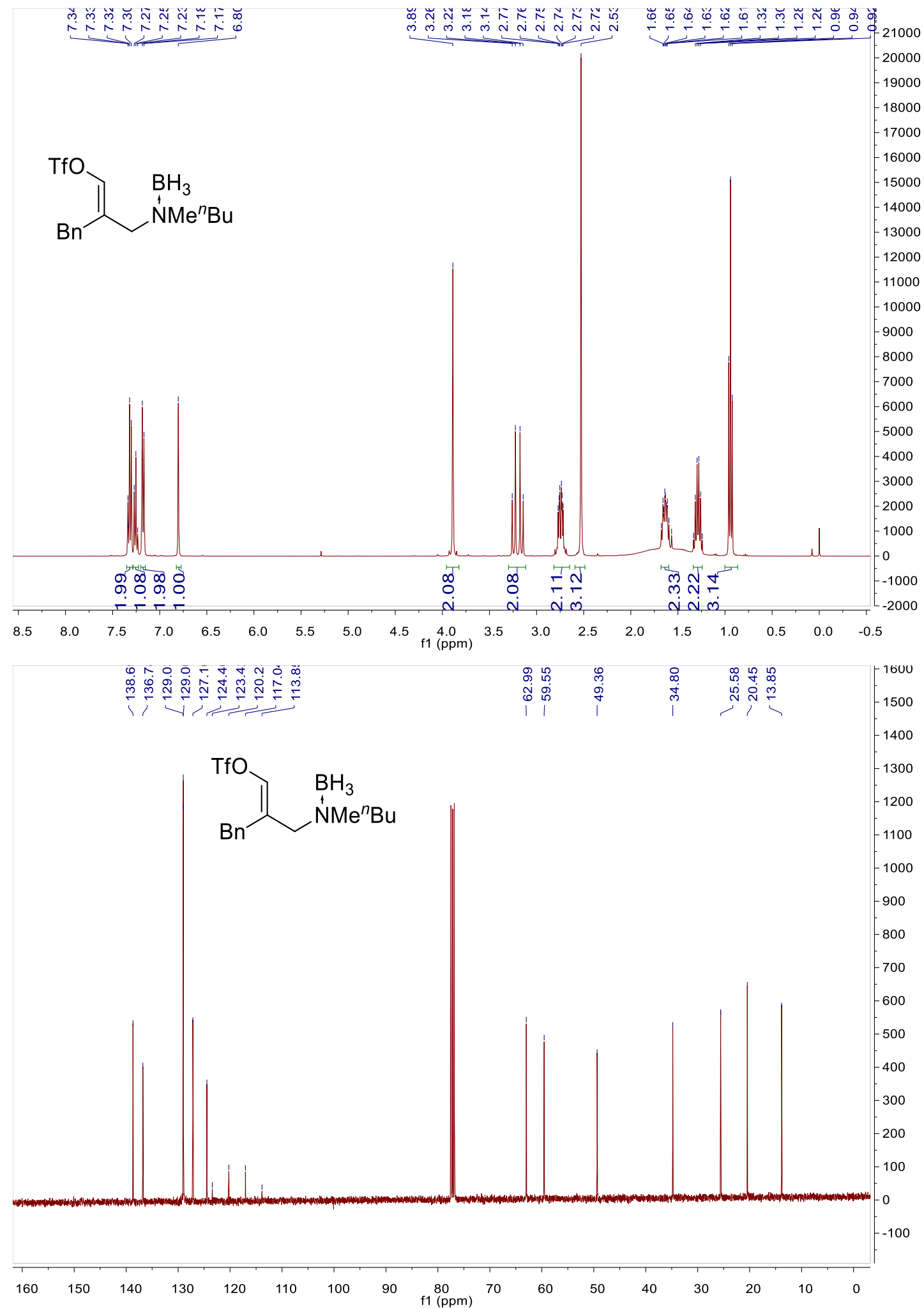




\section{(E)-2-benzyl-3-(benzyl(methyl)aminoborane)prop-1-en-1-yl}

\section{trifluoromethanesulfonate $(4 c)$}

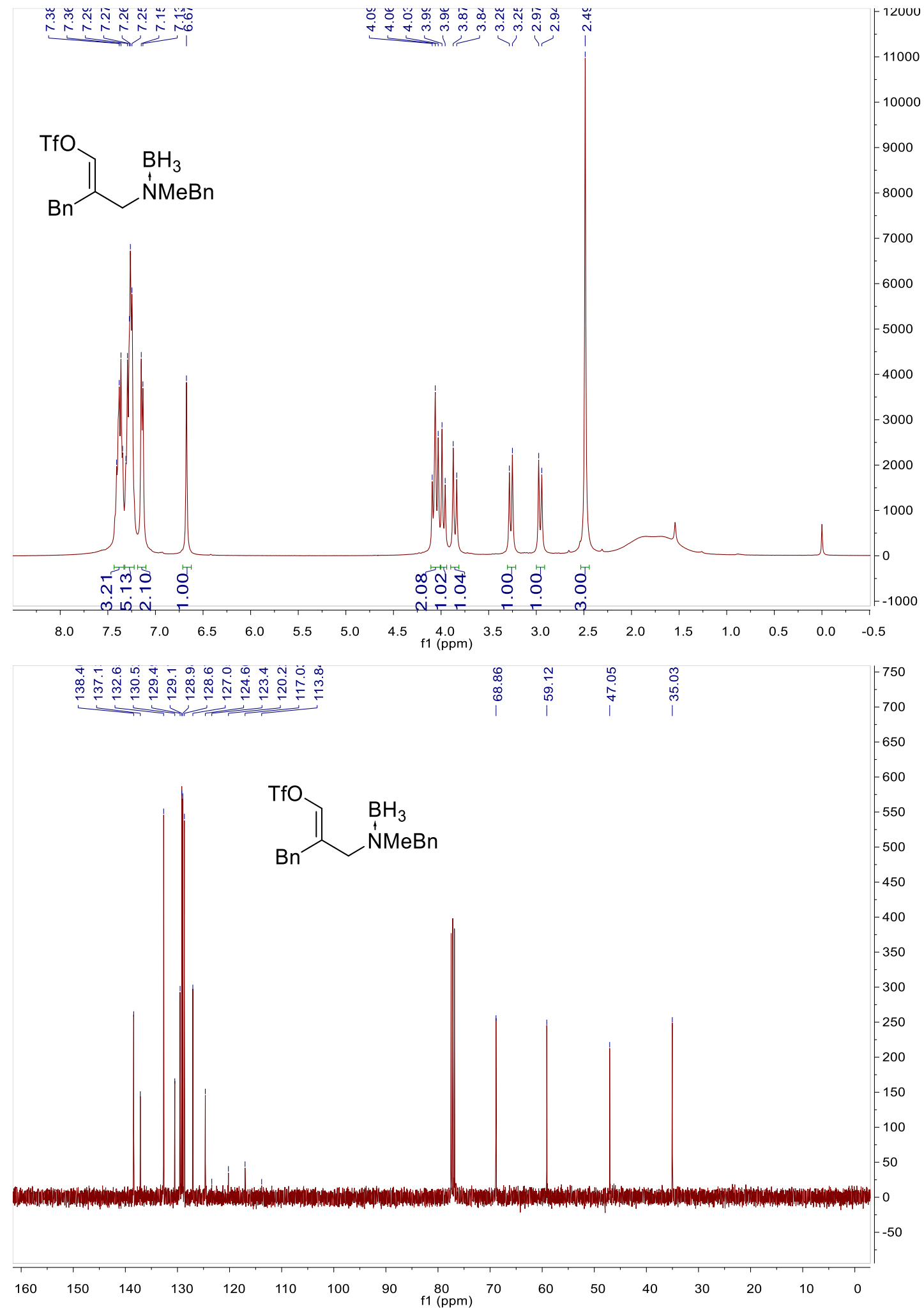



$((Z)-4 d)$

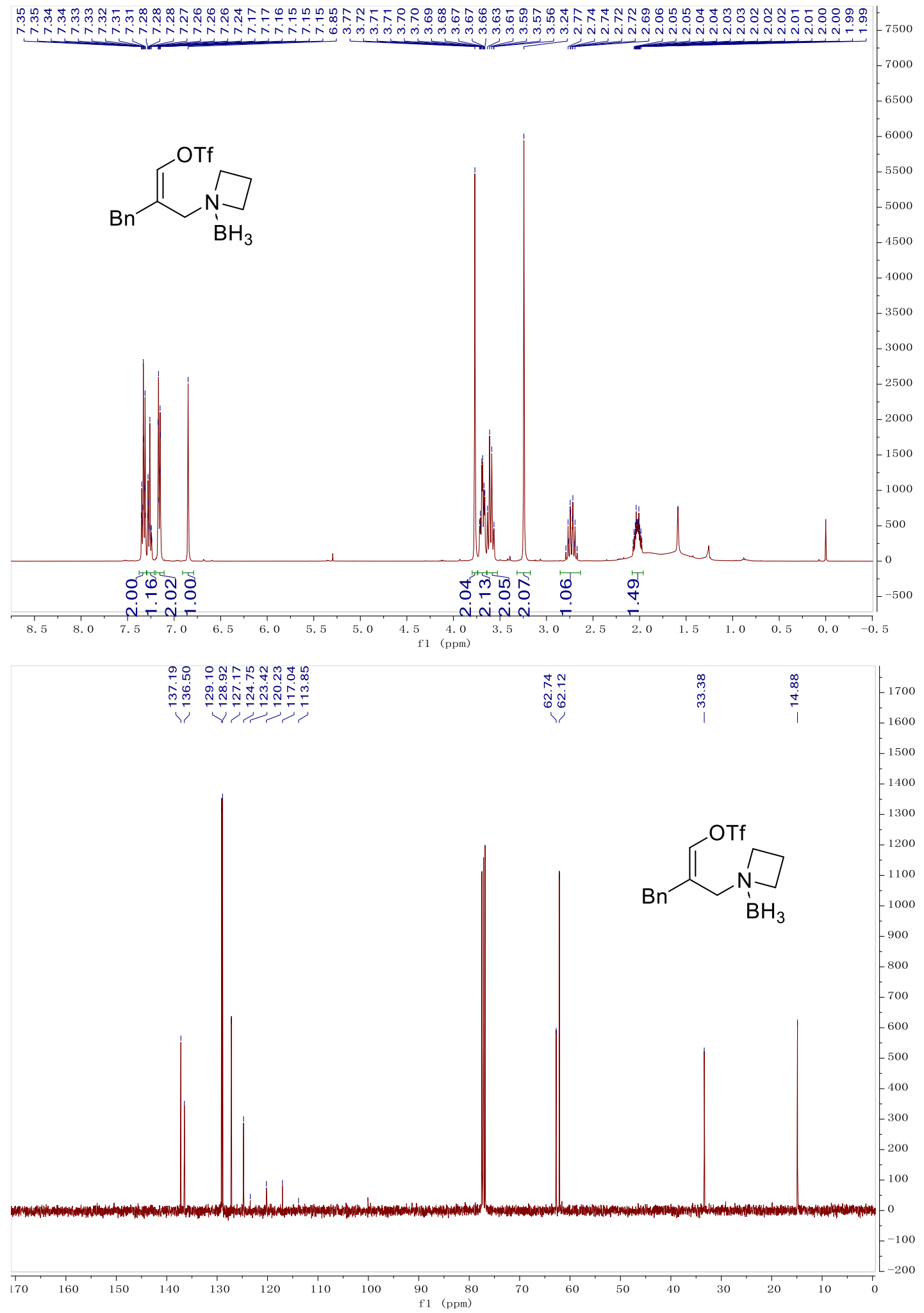



$((E)-4 d)$

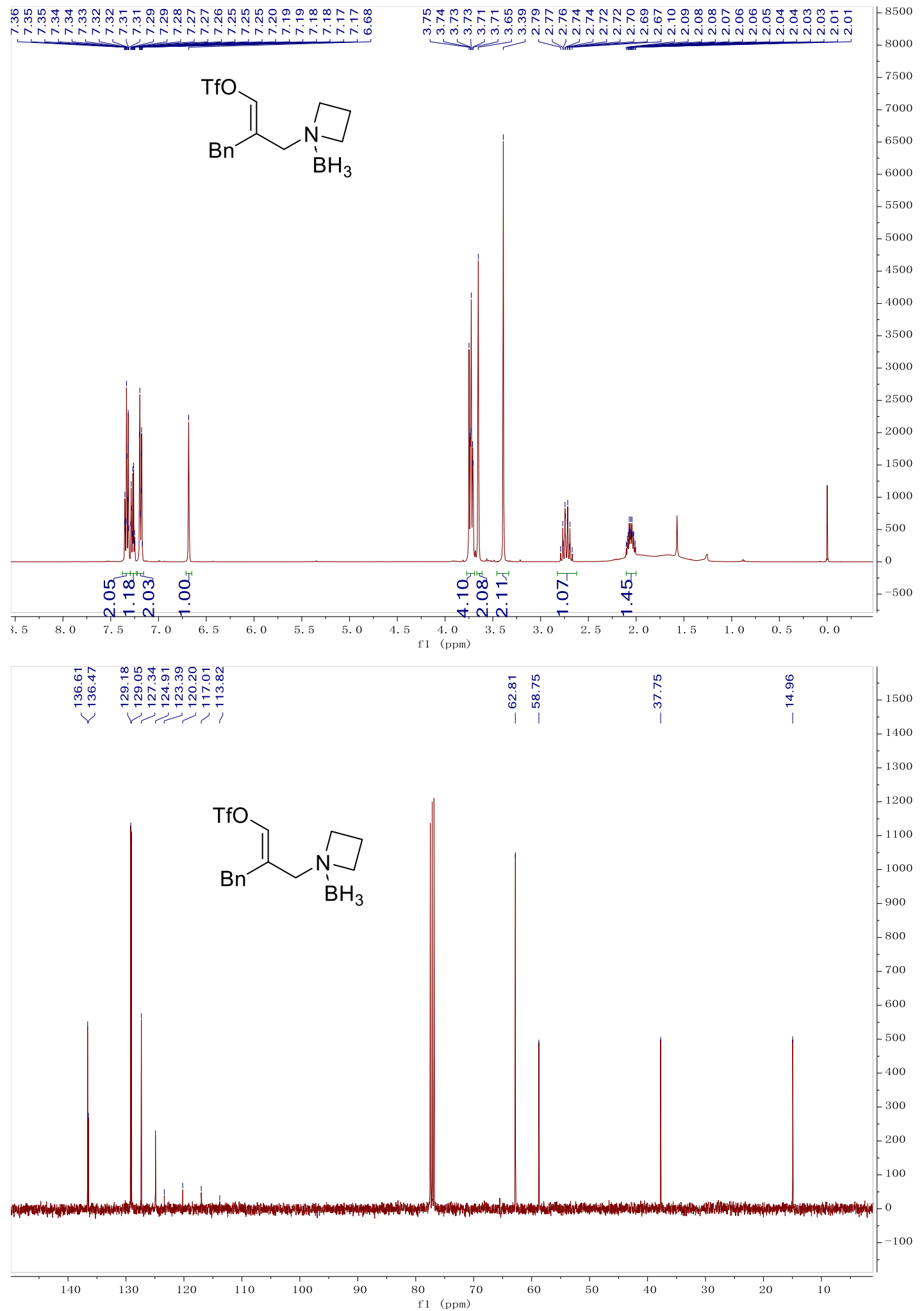



$((Z)-4 e)$

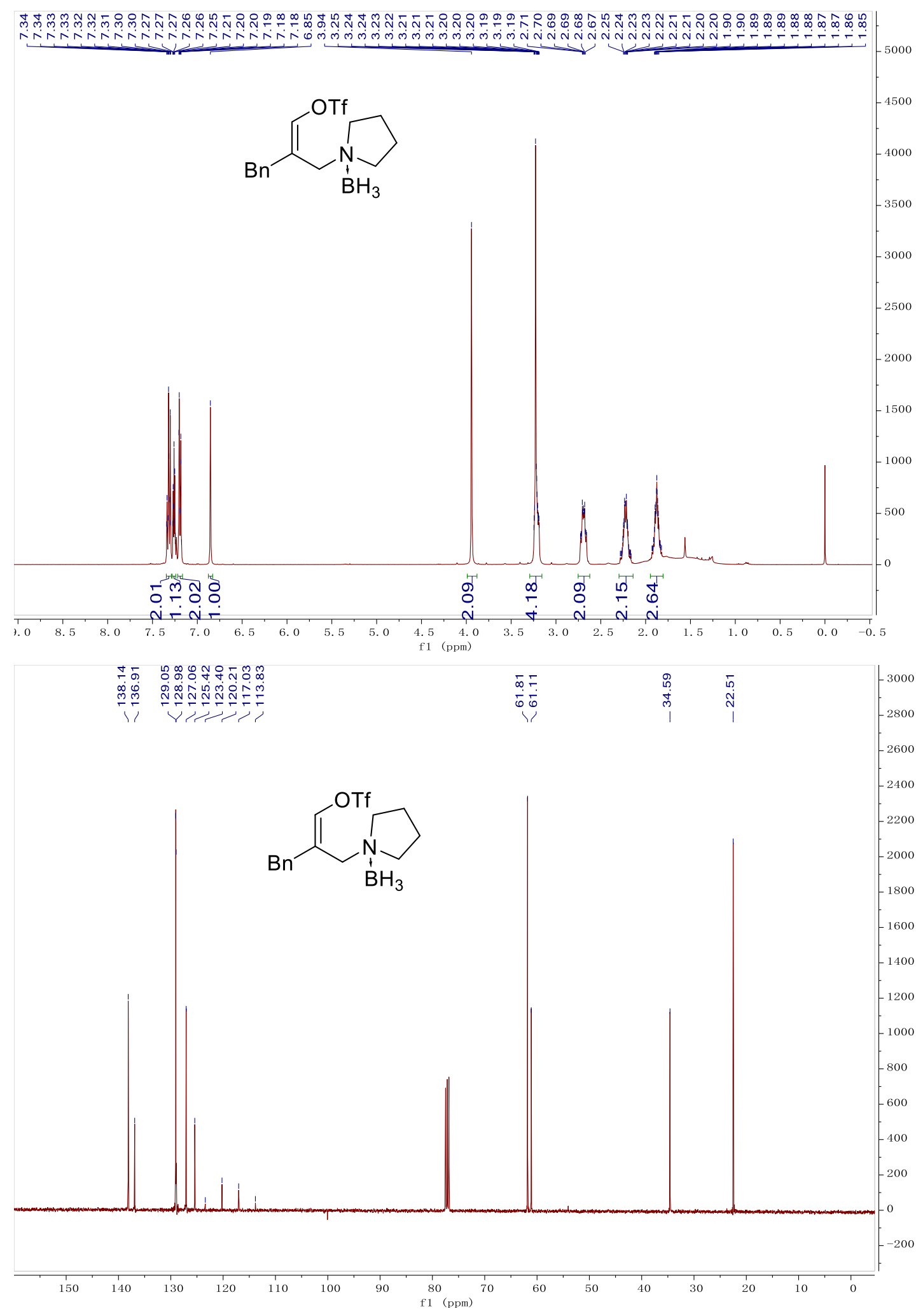



$((E)-4 e)$

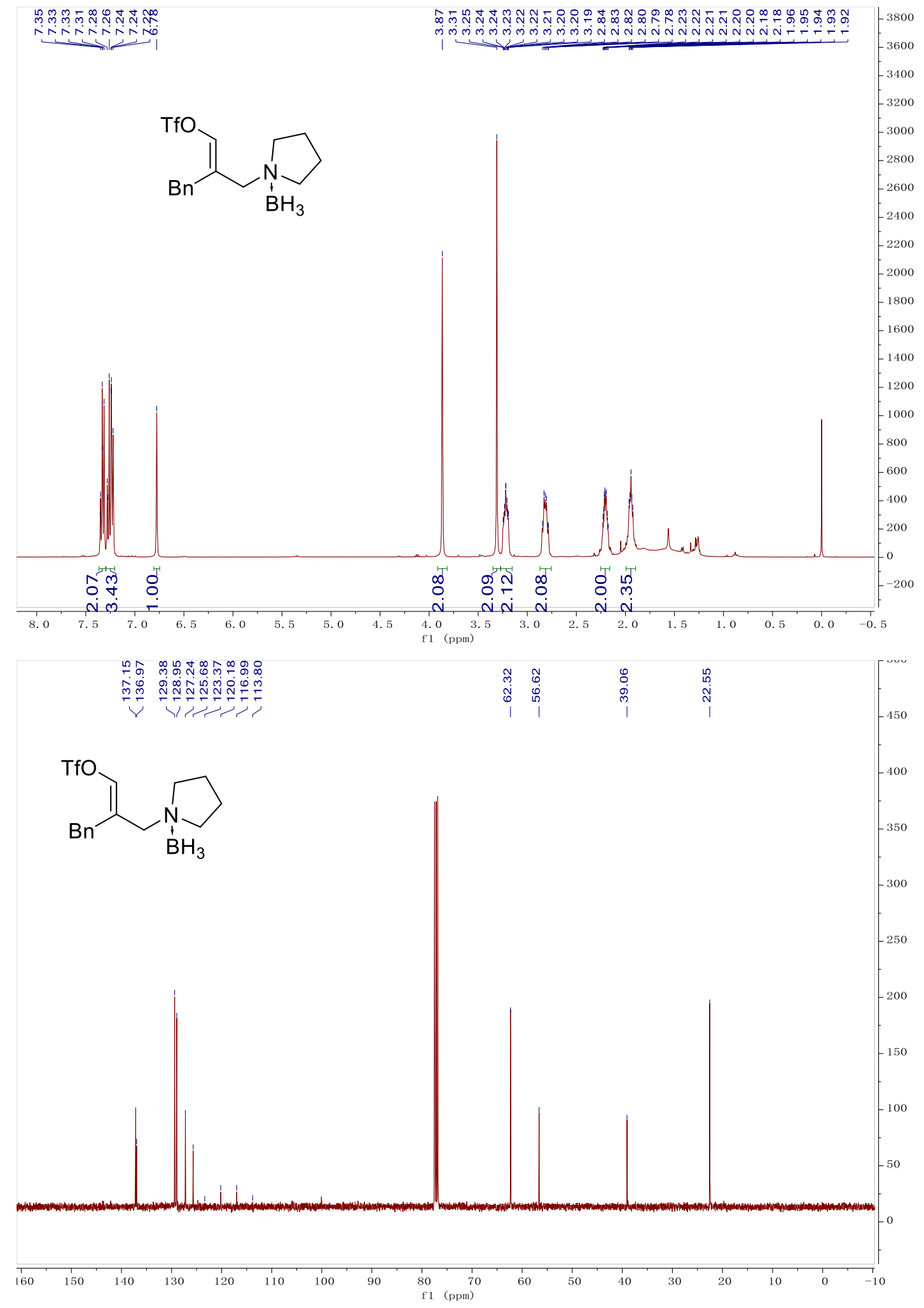




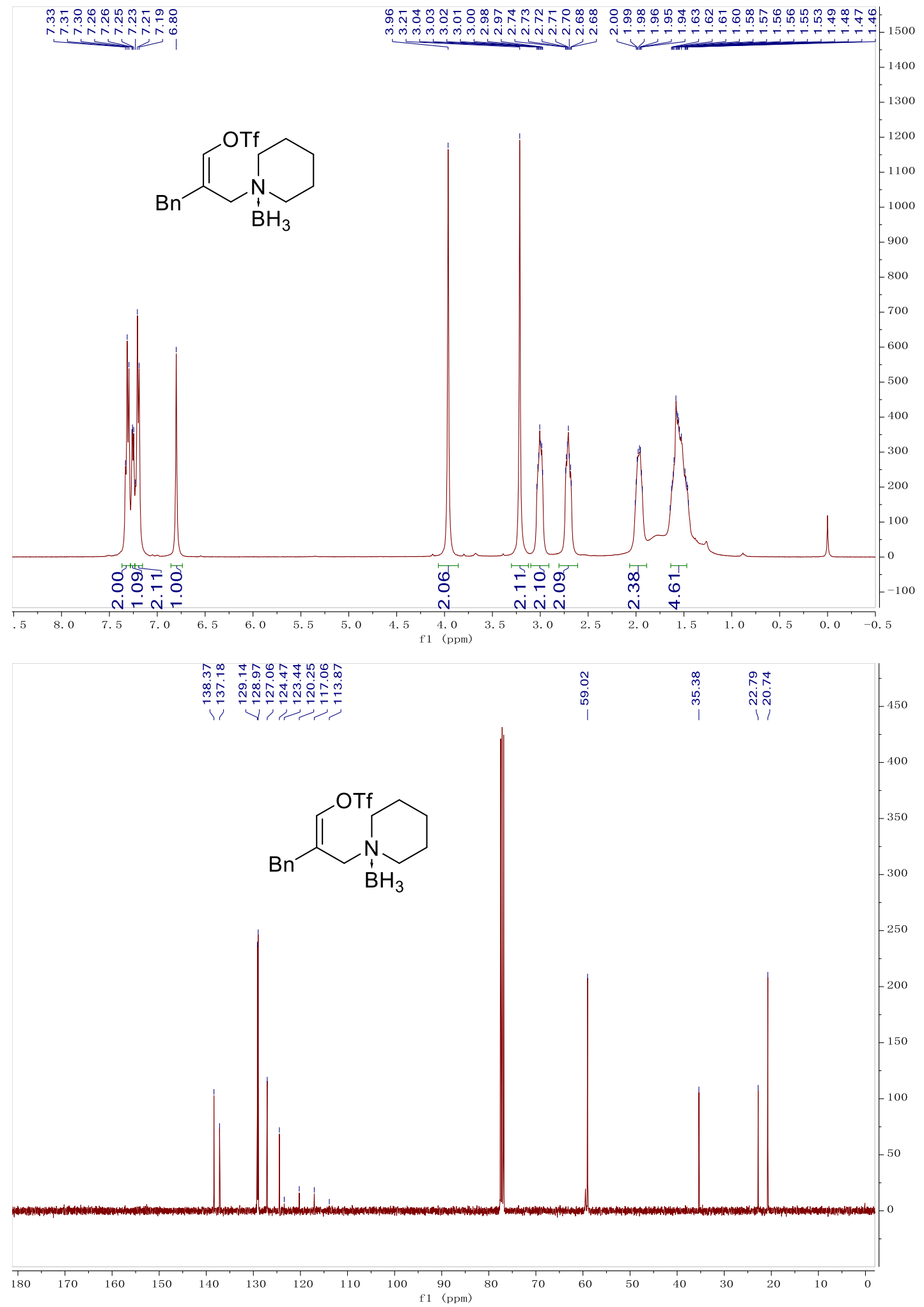



$((Z)-4 g)$

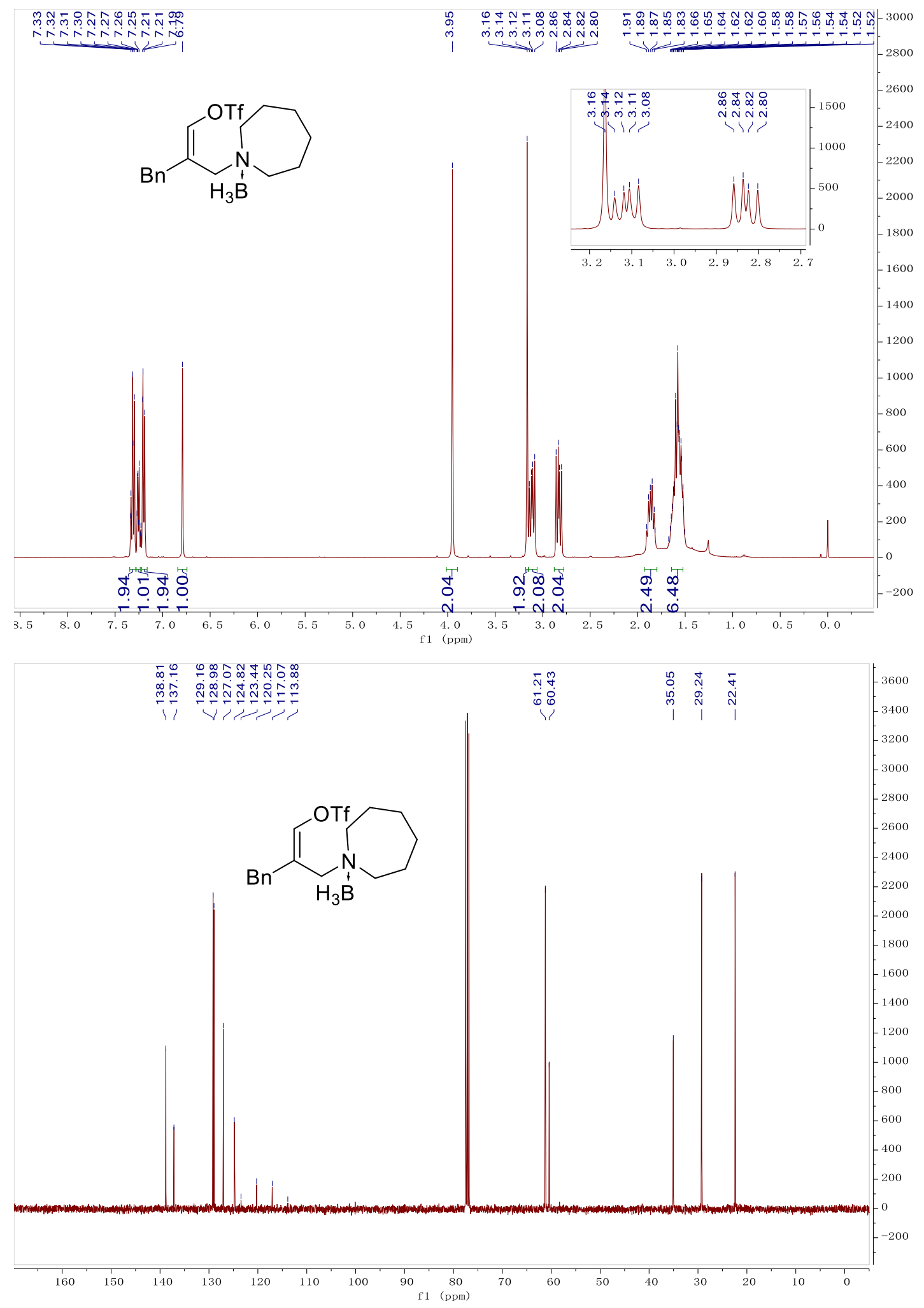



$((E)-4 g)$

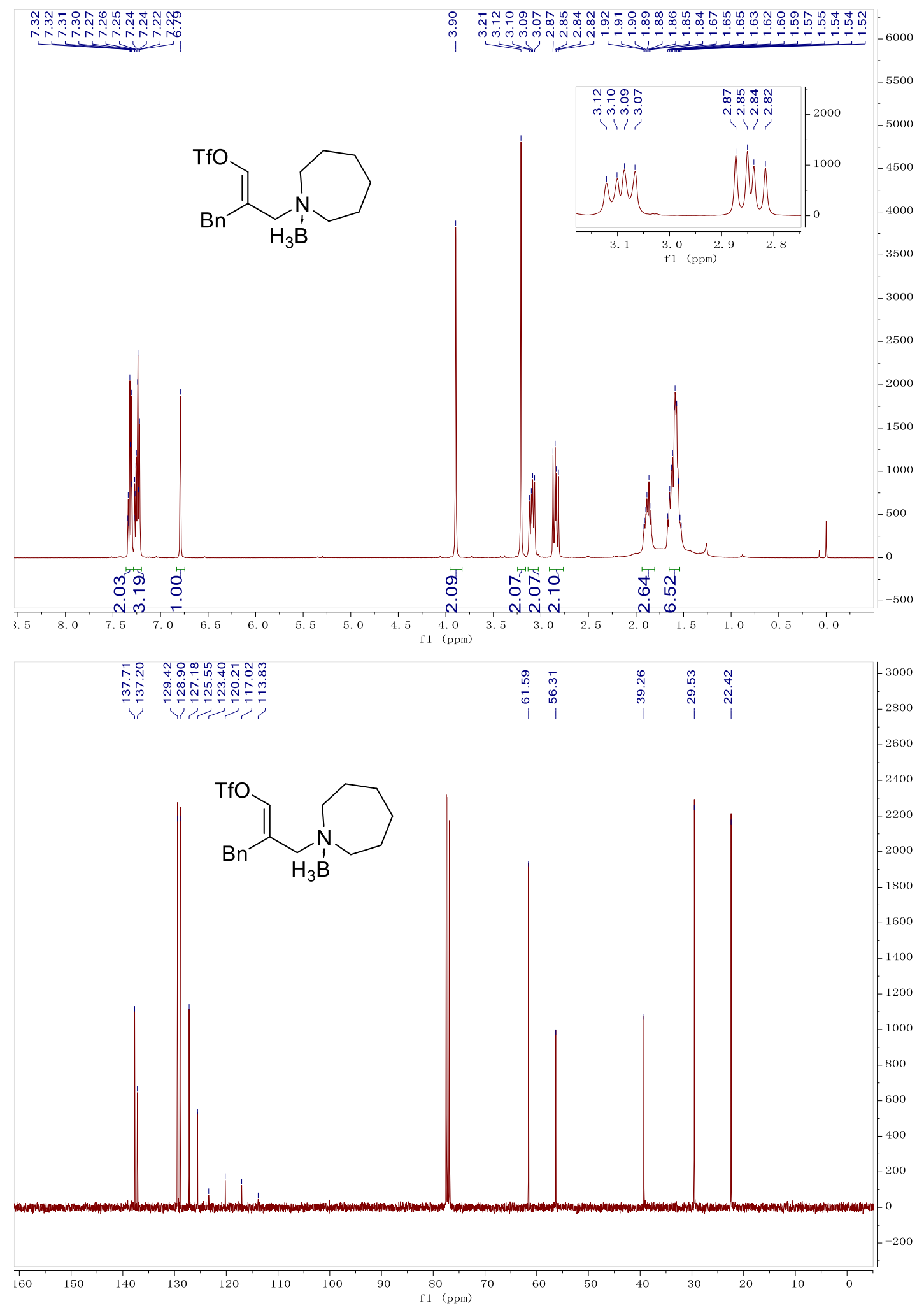


(4h)

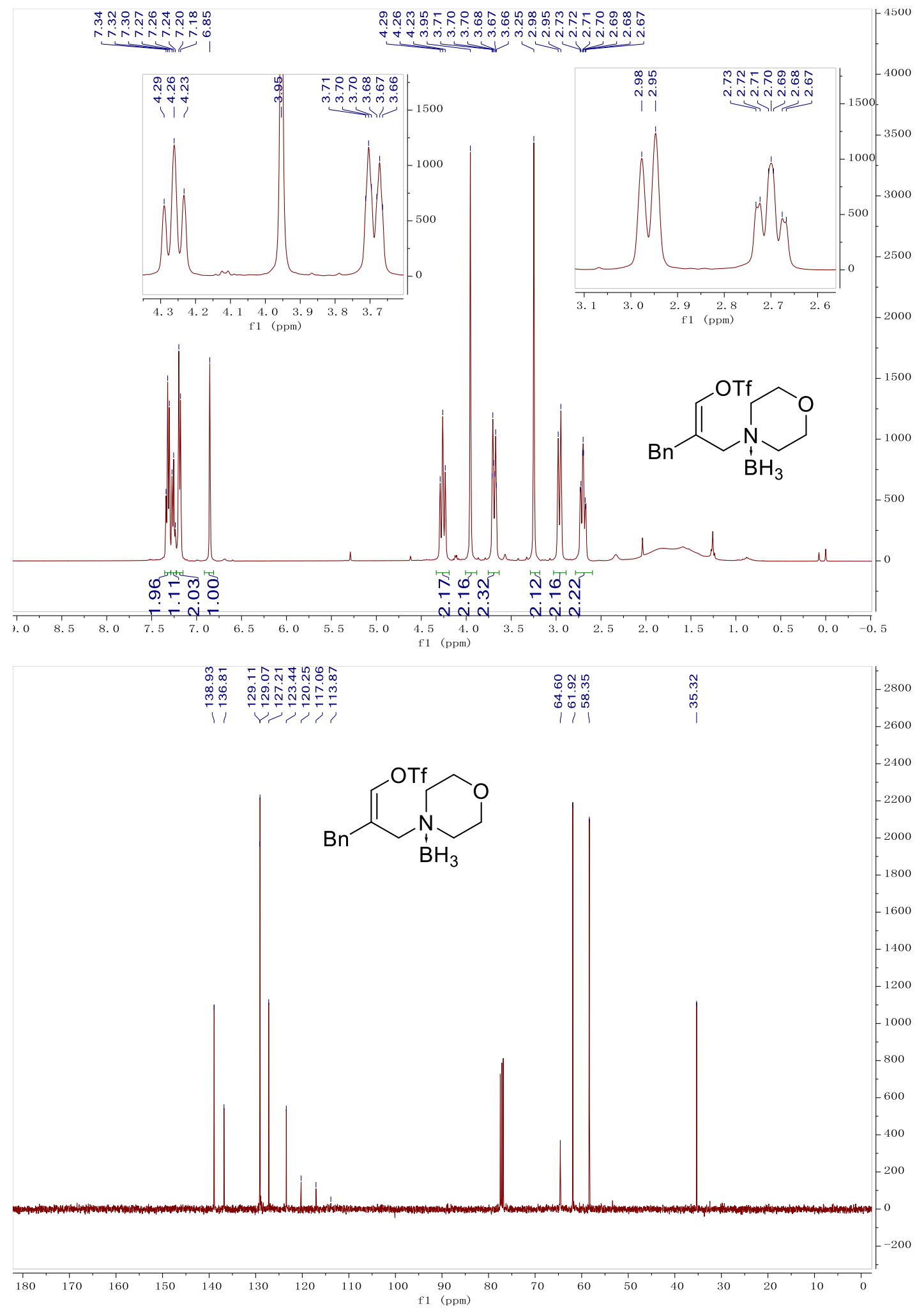



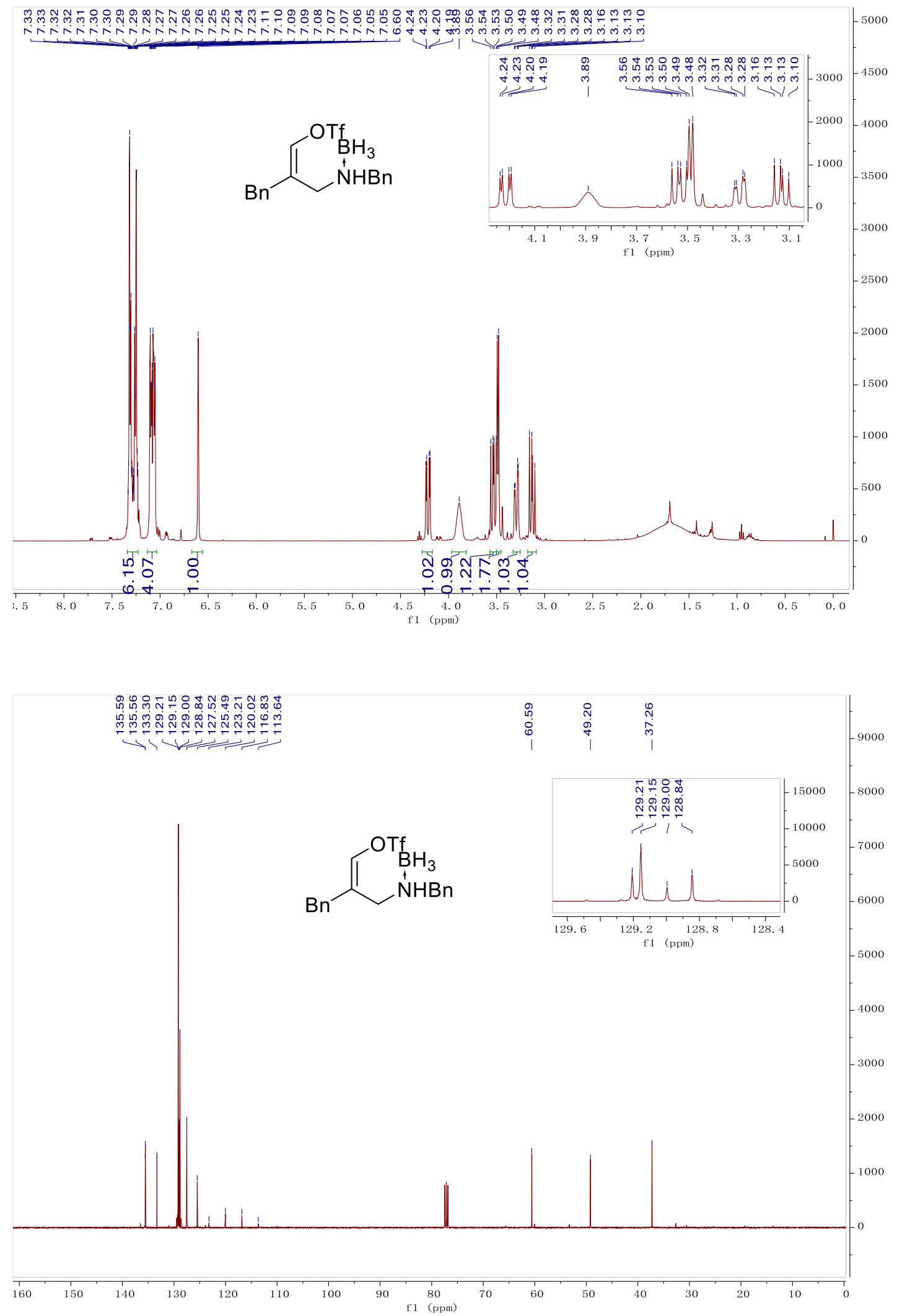


\section{trifluoromethanesulfonate $(4 \mathbf{j})$}

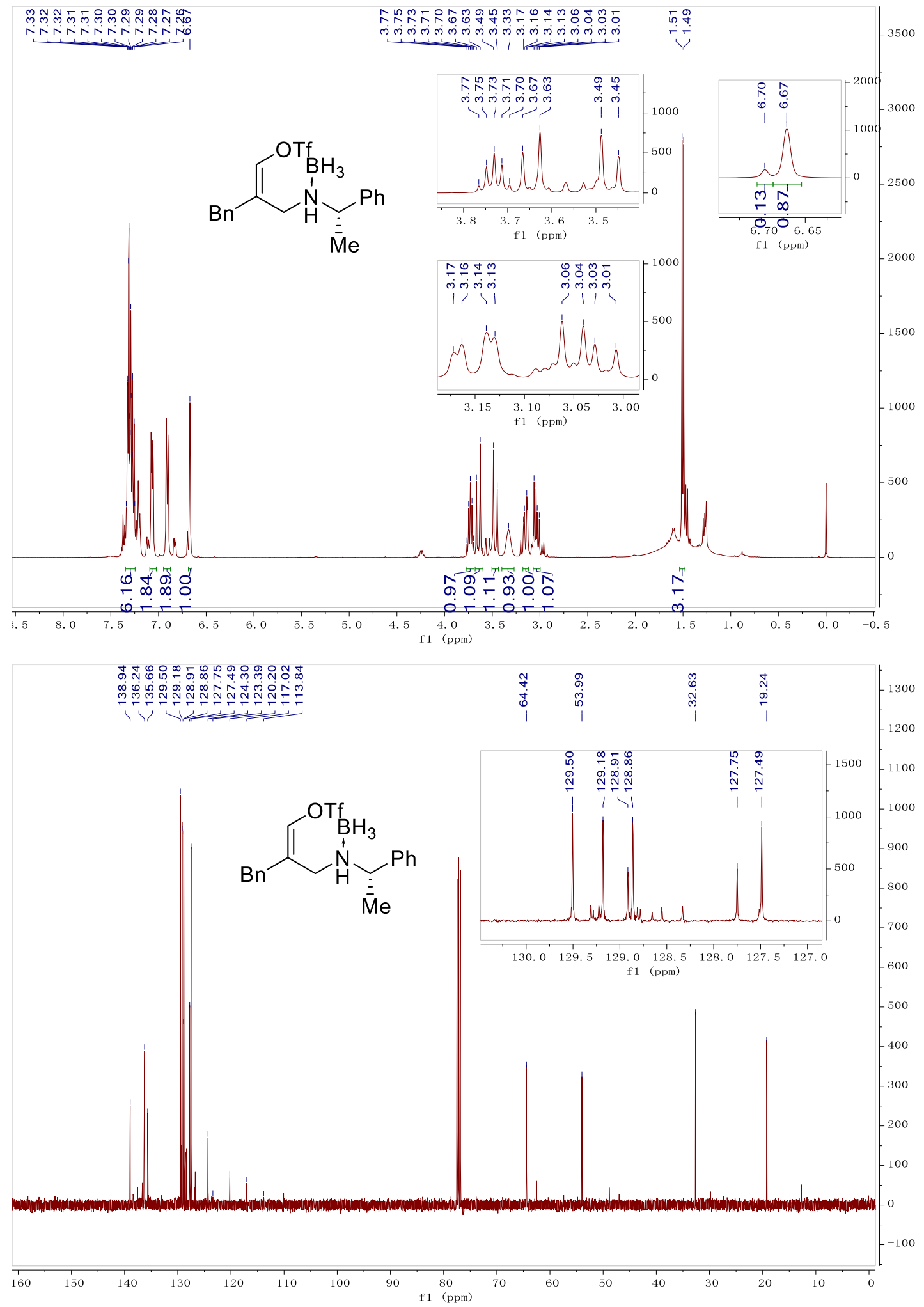


(Z)-2-benzyl- 3-((3S,4R)-3-((benzo[d][1,3]dioxol-5-yloxy)methyl)-4-(4-

fluorophenyl)piperidin-1-yl aminoborane)prop-1-en-1-yl

trifluoromethanesulfonate $(4 k)$

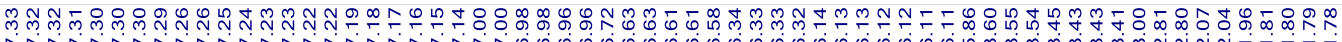

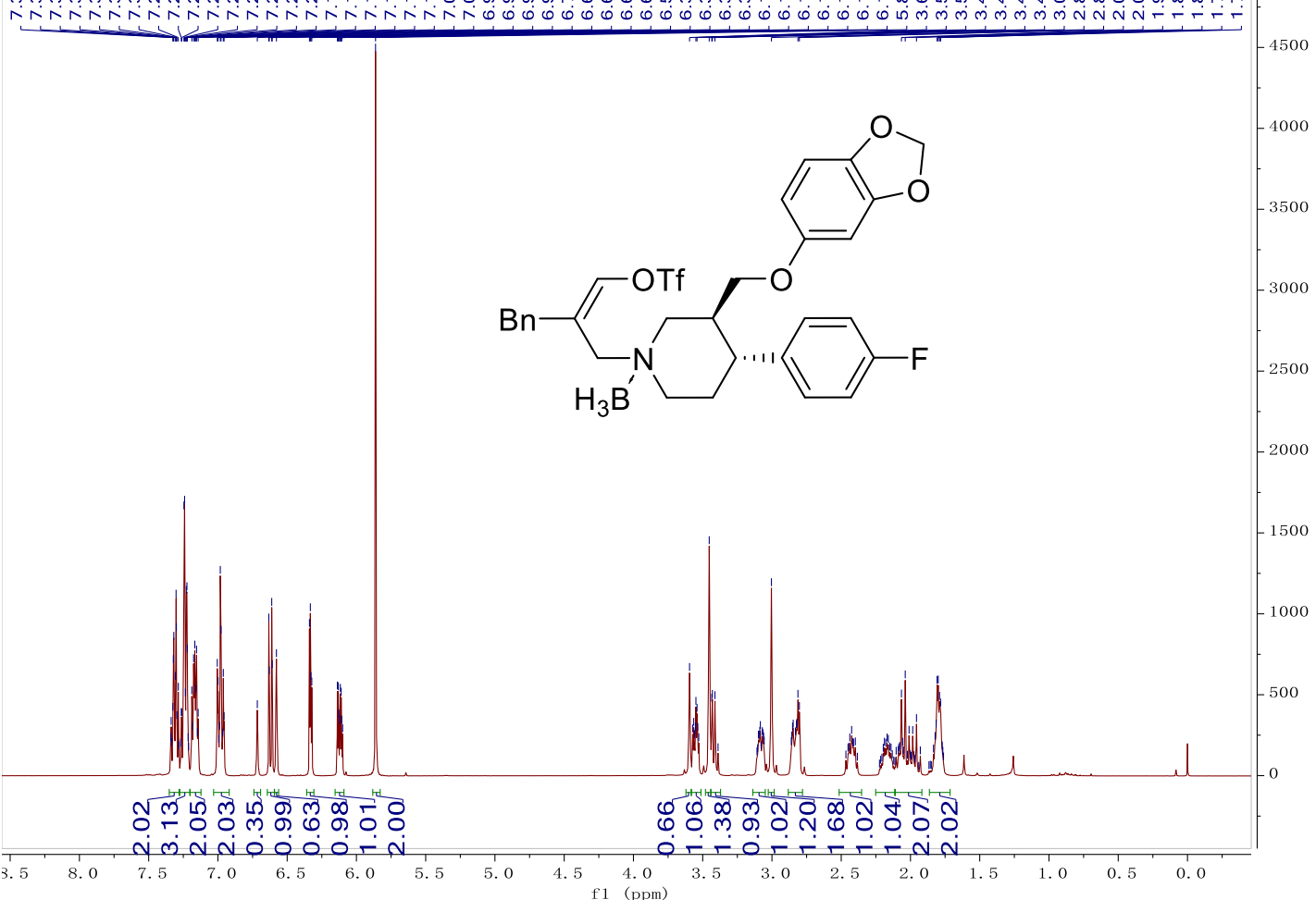

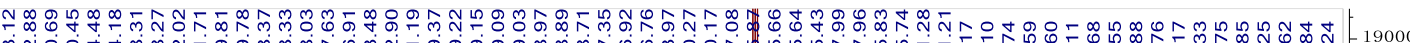

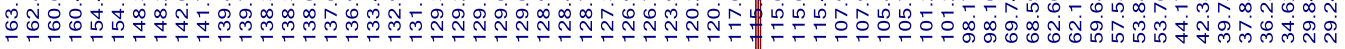

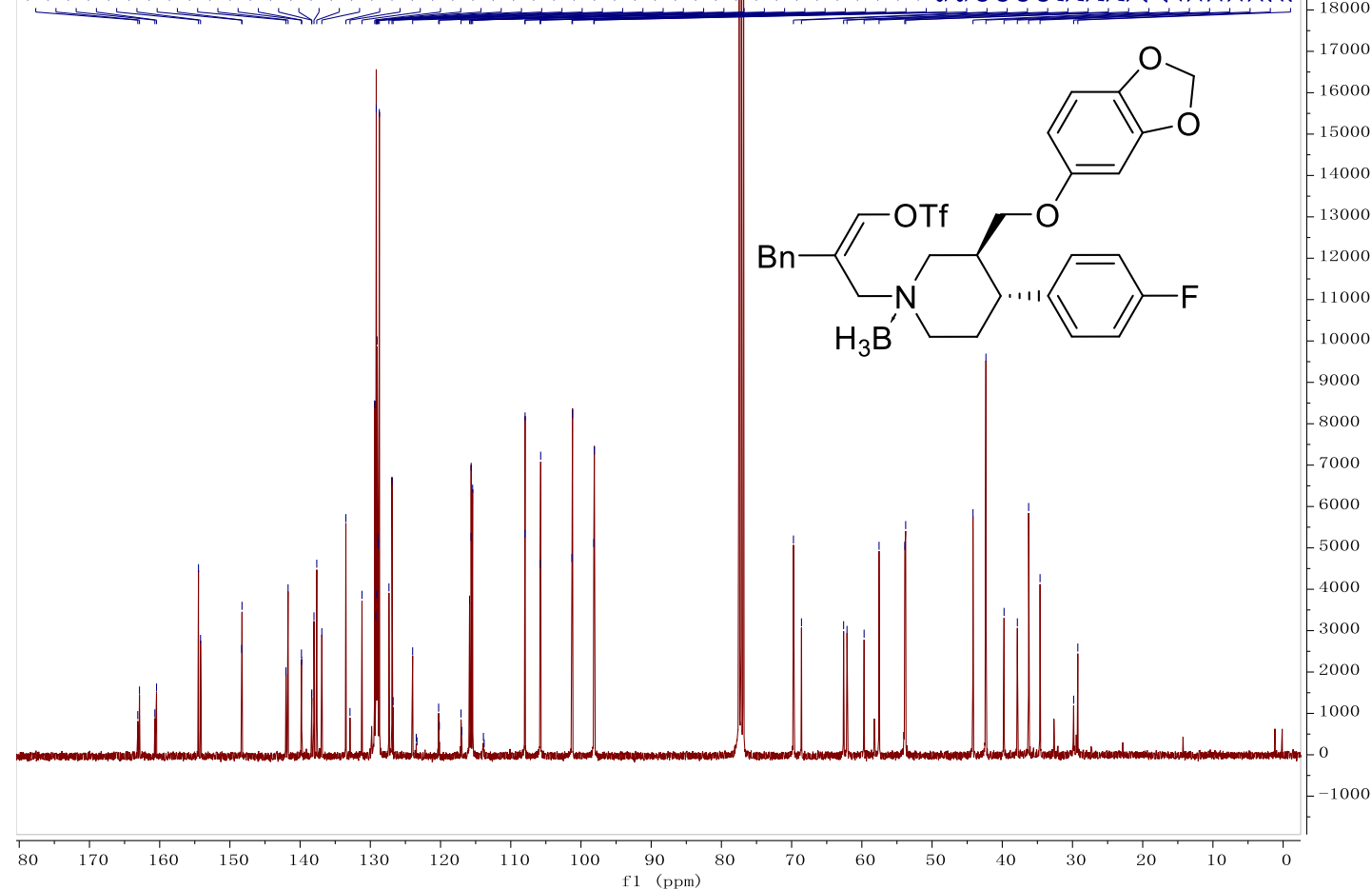


(Z)-2-benzyl-3-((3-(10,11-dihydro-5H-dibenzo[ $[a, d][7]$ annulen-5-

ylidene)propyl)(methyl)aminoborane)prop-1-en-1-yl trifluoromethanesulfonate (4I)

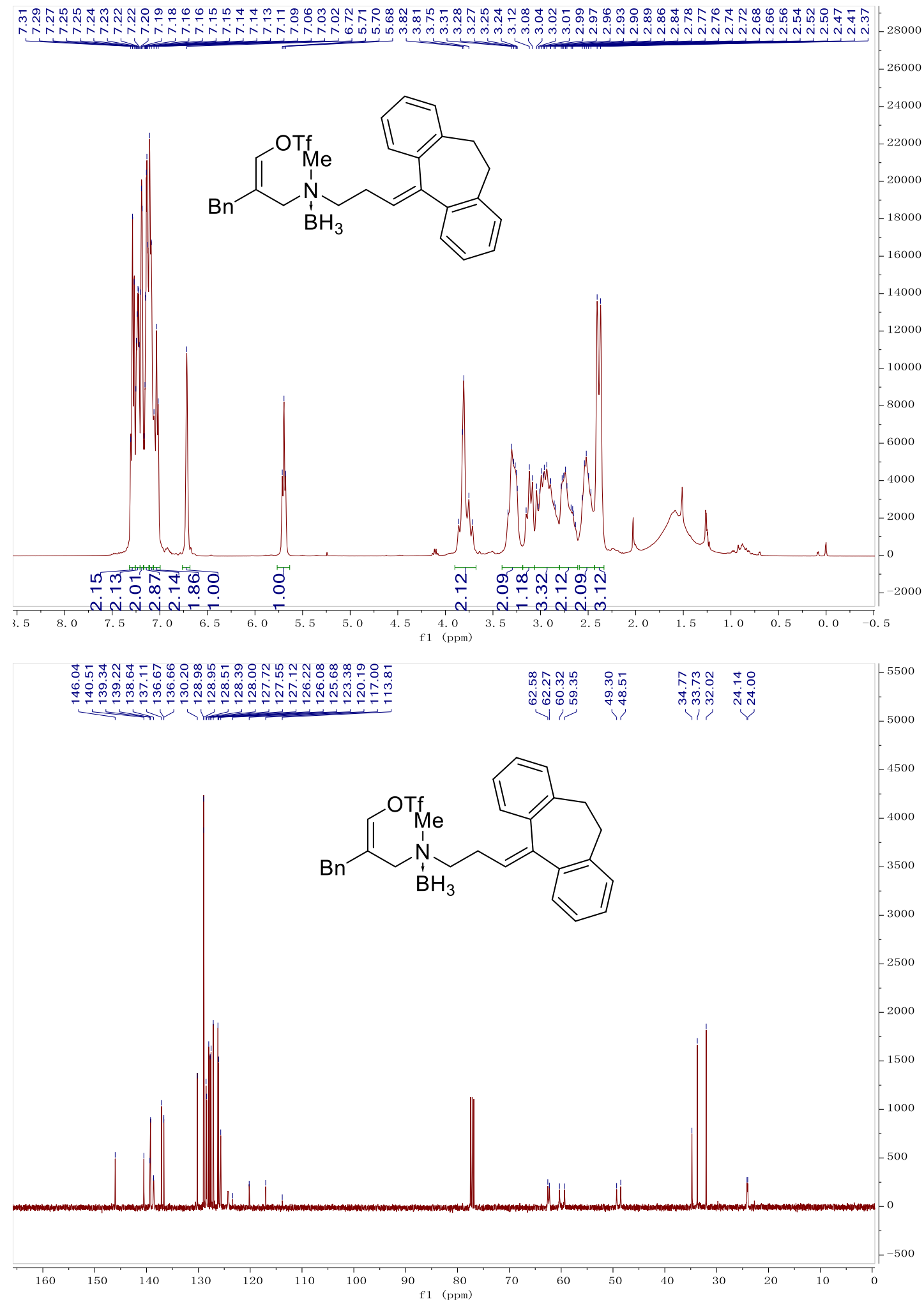




\section{(Z)-2-benzyl-3-(methyl(3-phenyl-3-(4-}

(trifluoromethyl)phenoxy)propyl)aminoborane)prop-1-en-1-yl

\section{trifluoromethanesulfonate $(4 \mathrm{~m})$}

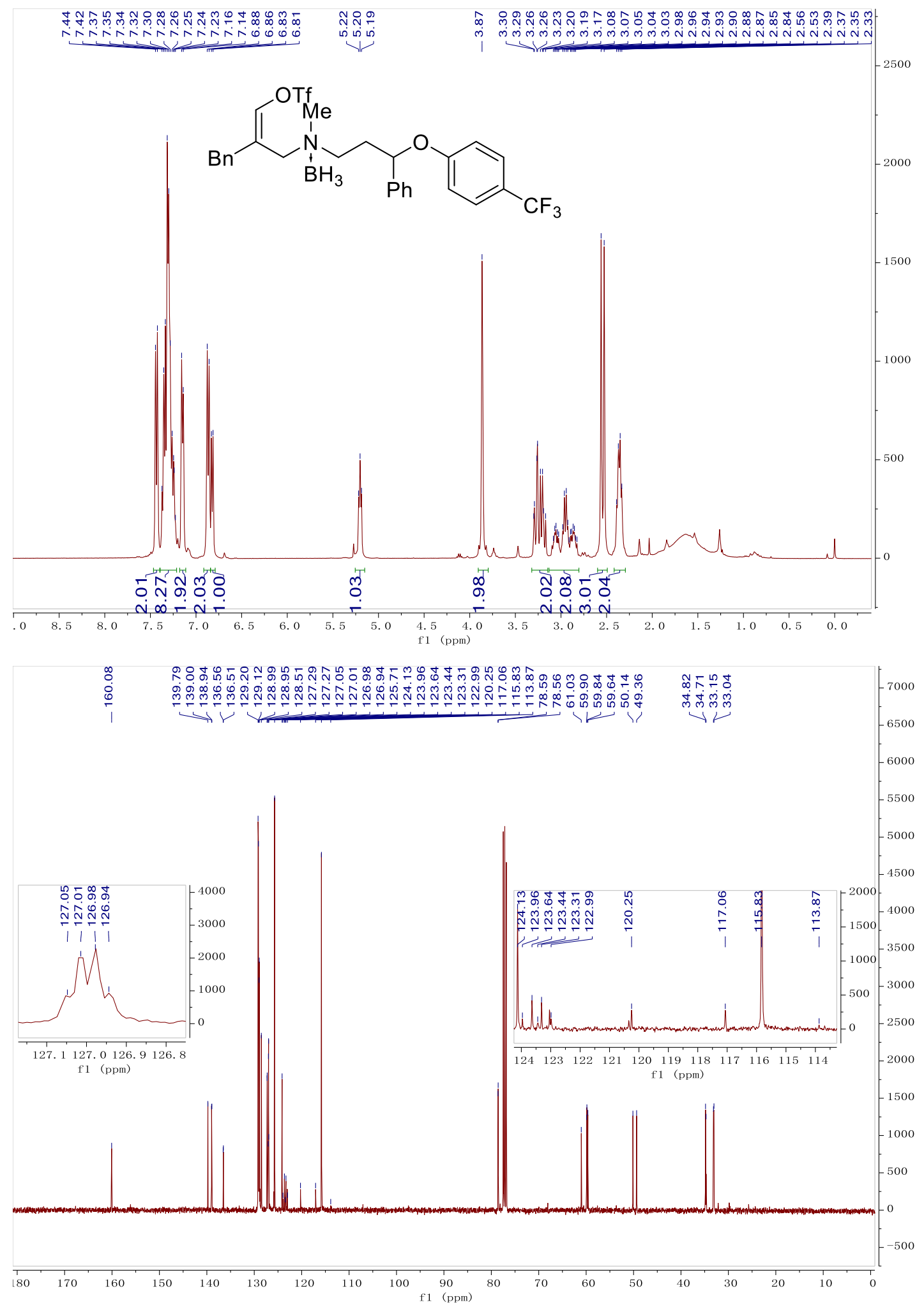




\section{(E)-3-(benzyl(methyl)aminoborane)-2-(4-chlorobenzyl)prop-1-en-1-yl}

\section{trifluoromethanesulfonate $(4 n)$}

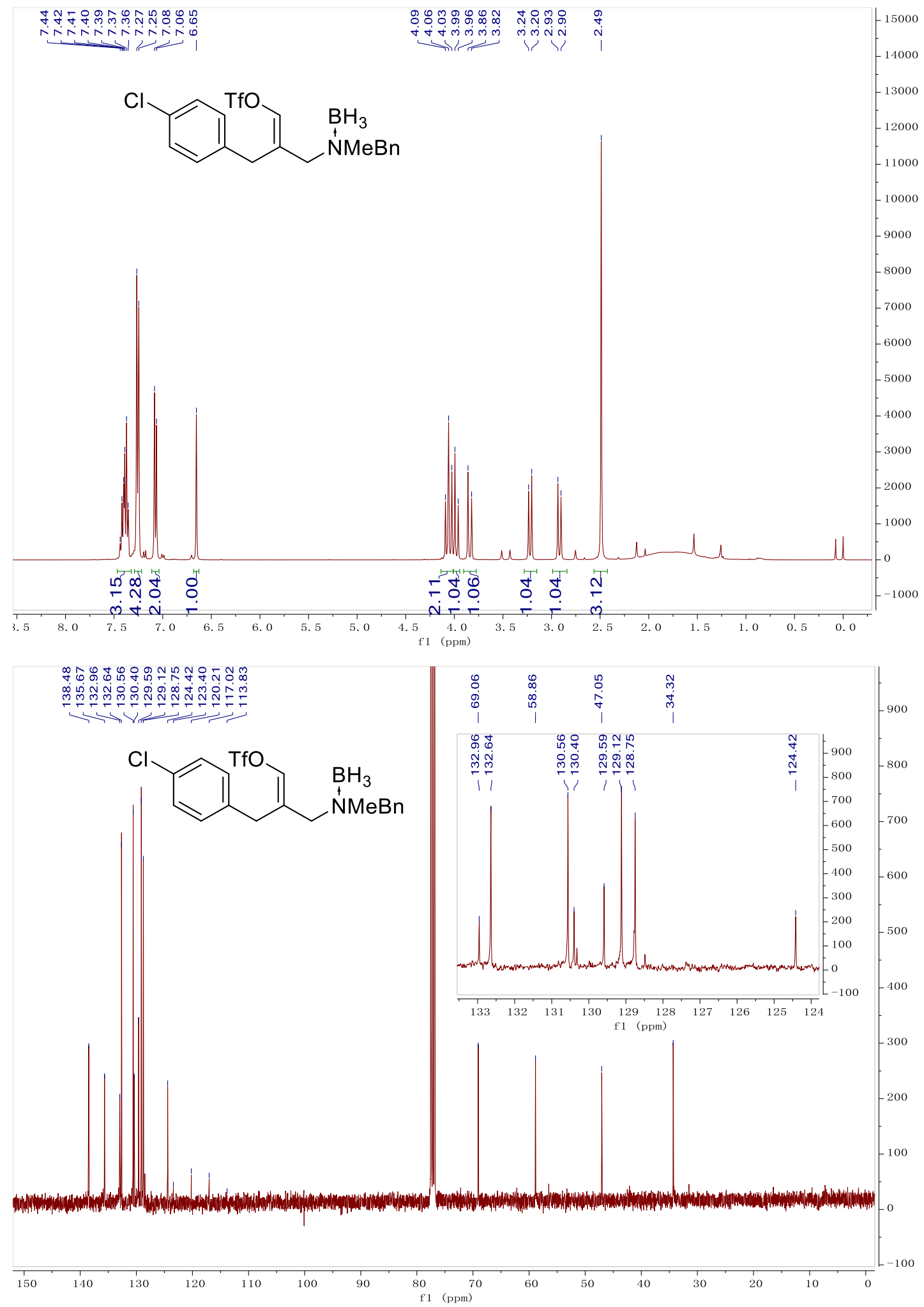




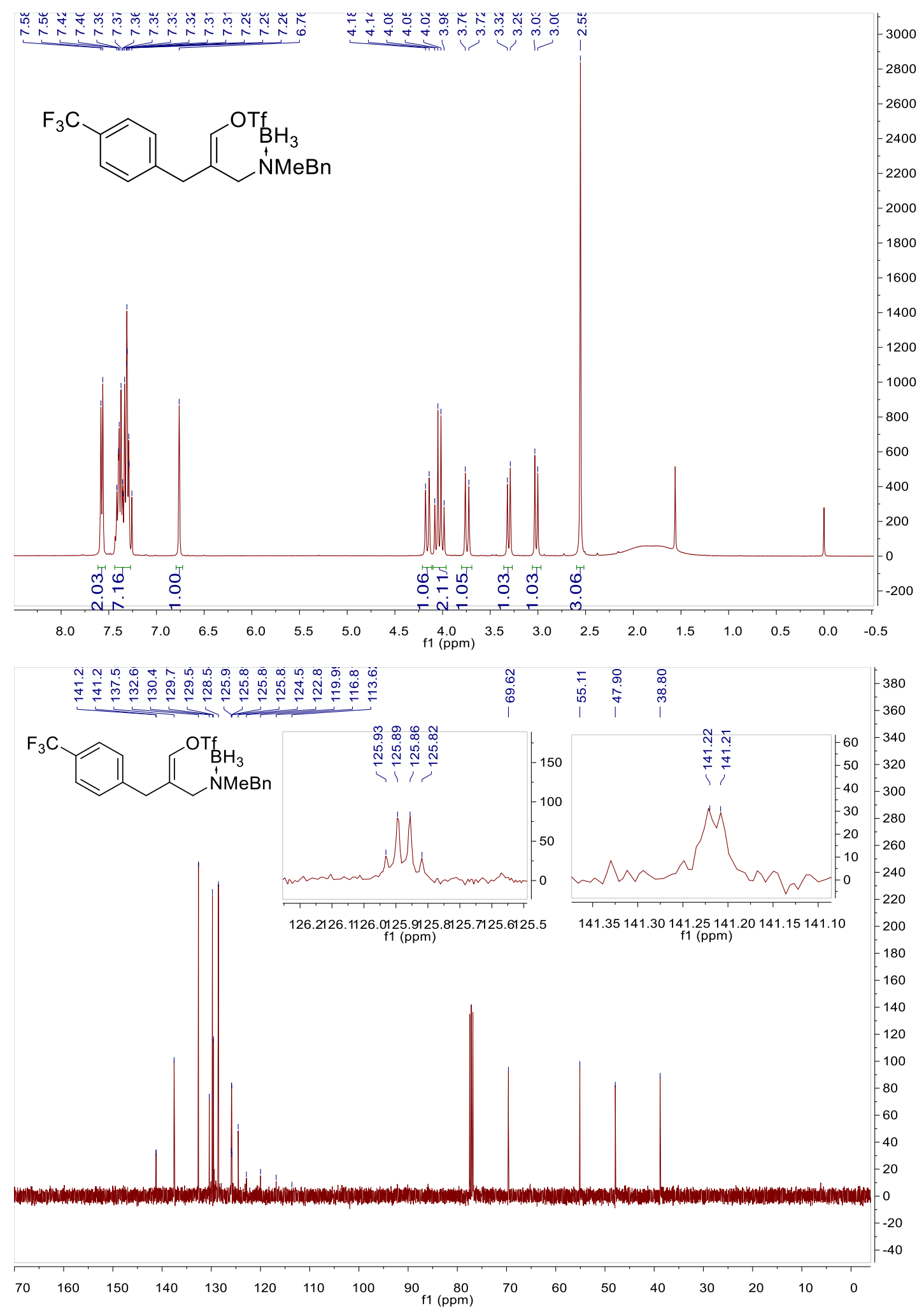



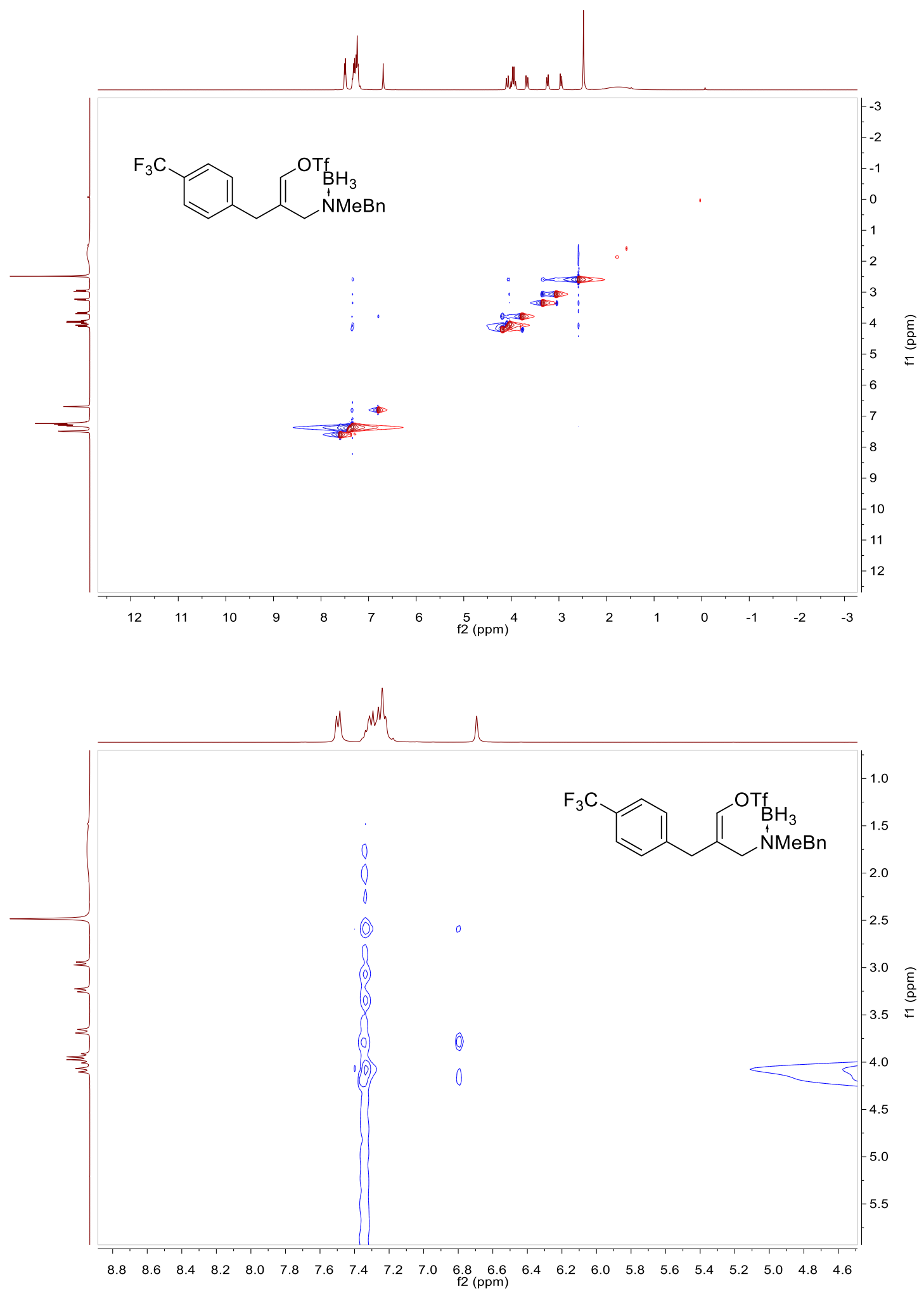

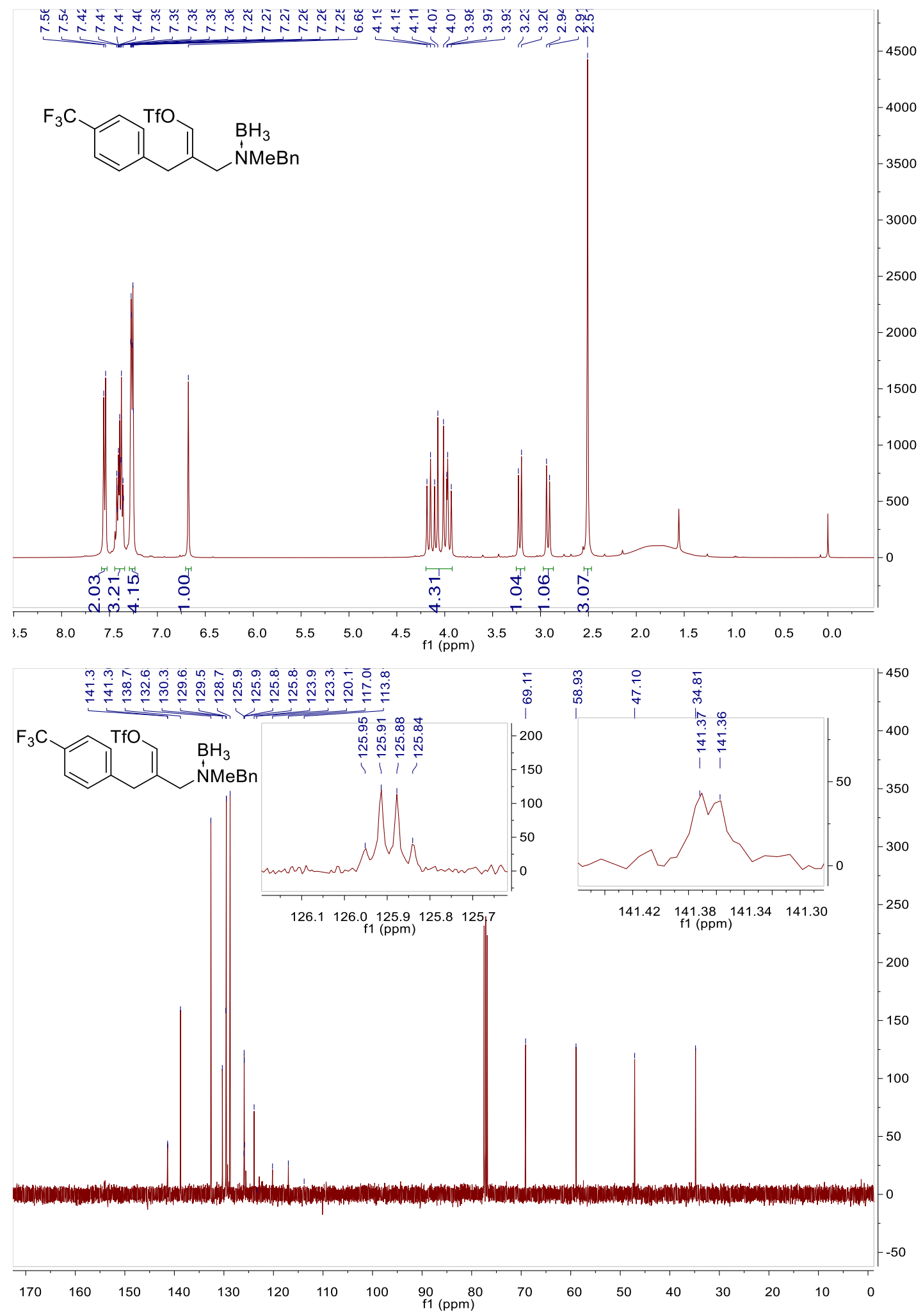

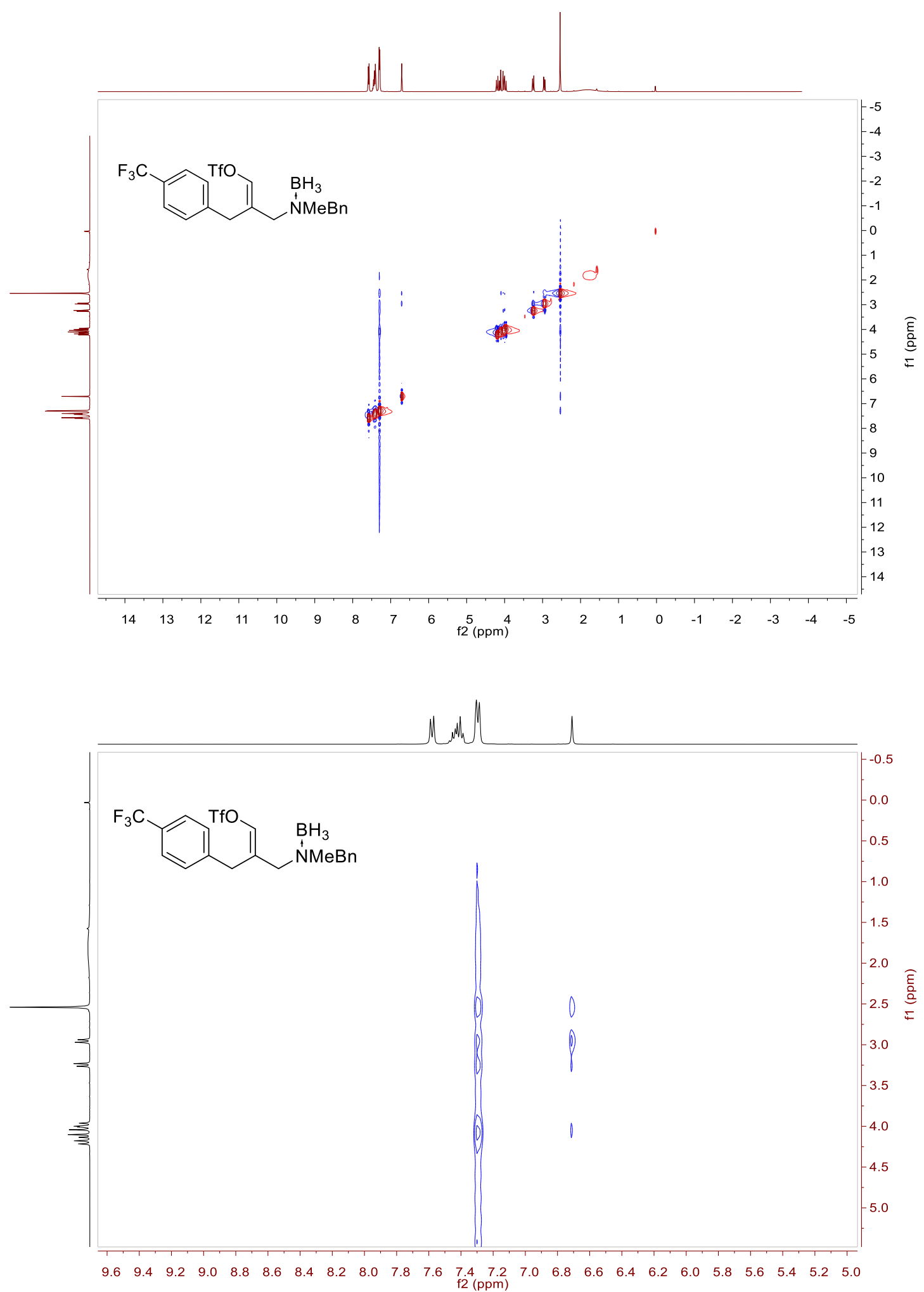


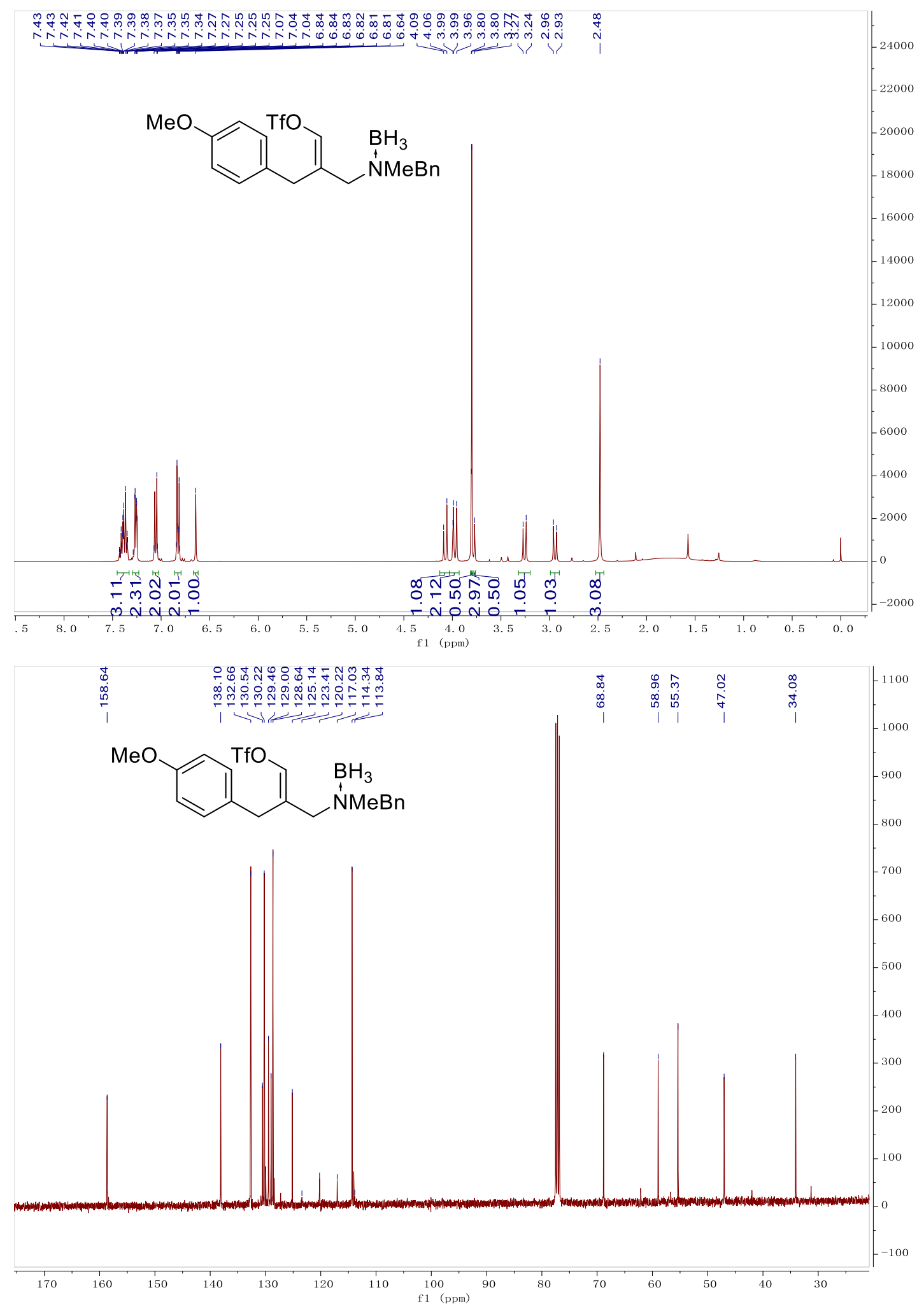




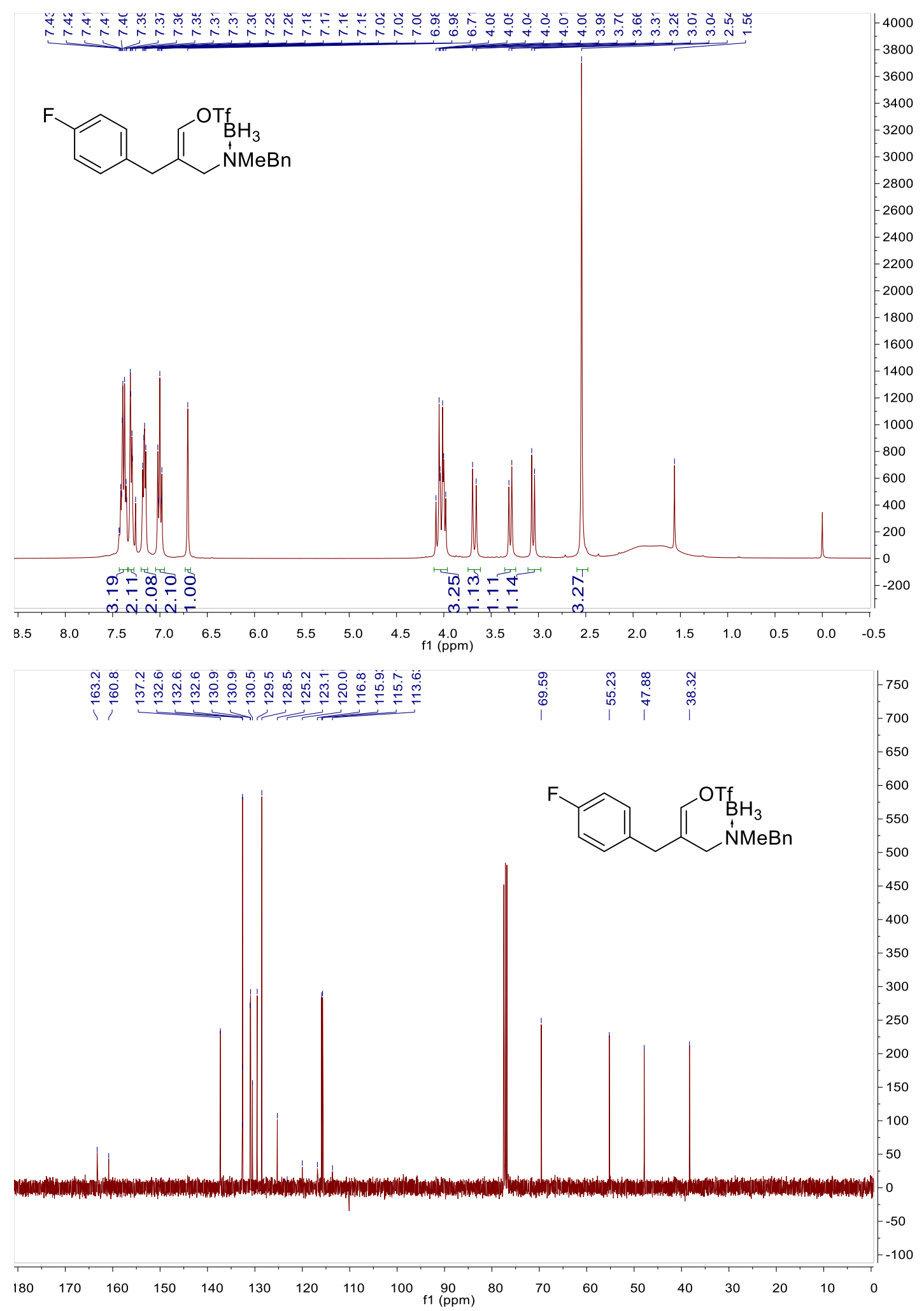




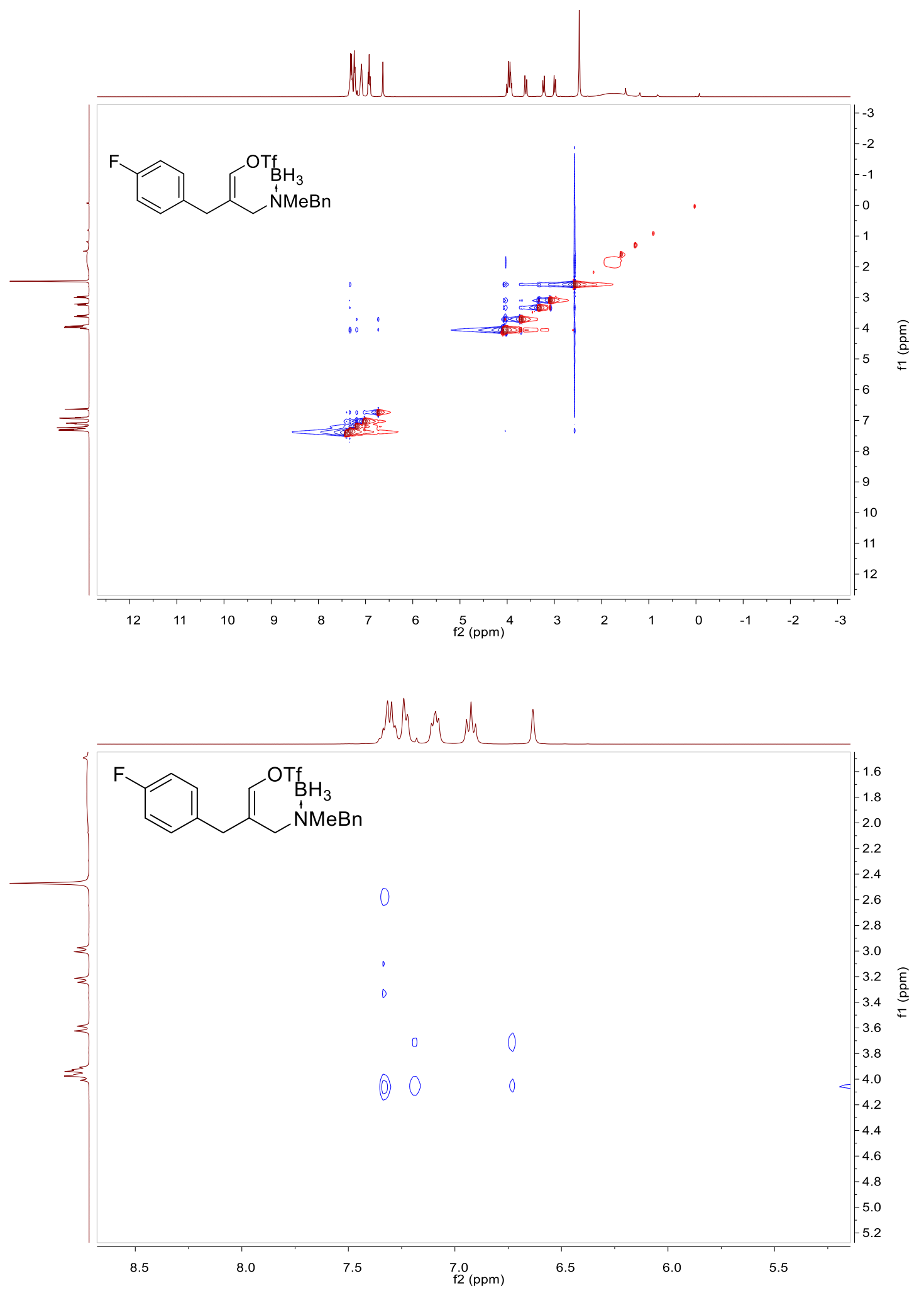




\section{(E)-3-(benzyl(methyl)aminoborane)-2-(4-fluorobenzyl)prop-1-en-1-yl}

trifluoromethanesulfonate $((E)-4 q)$

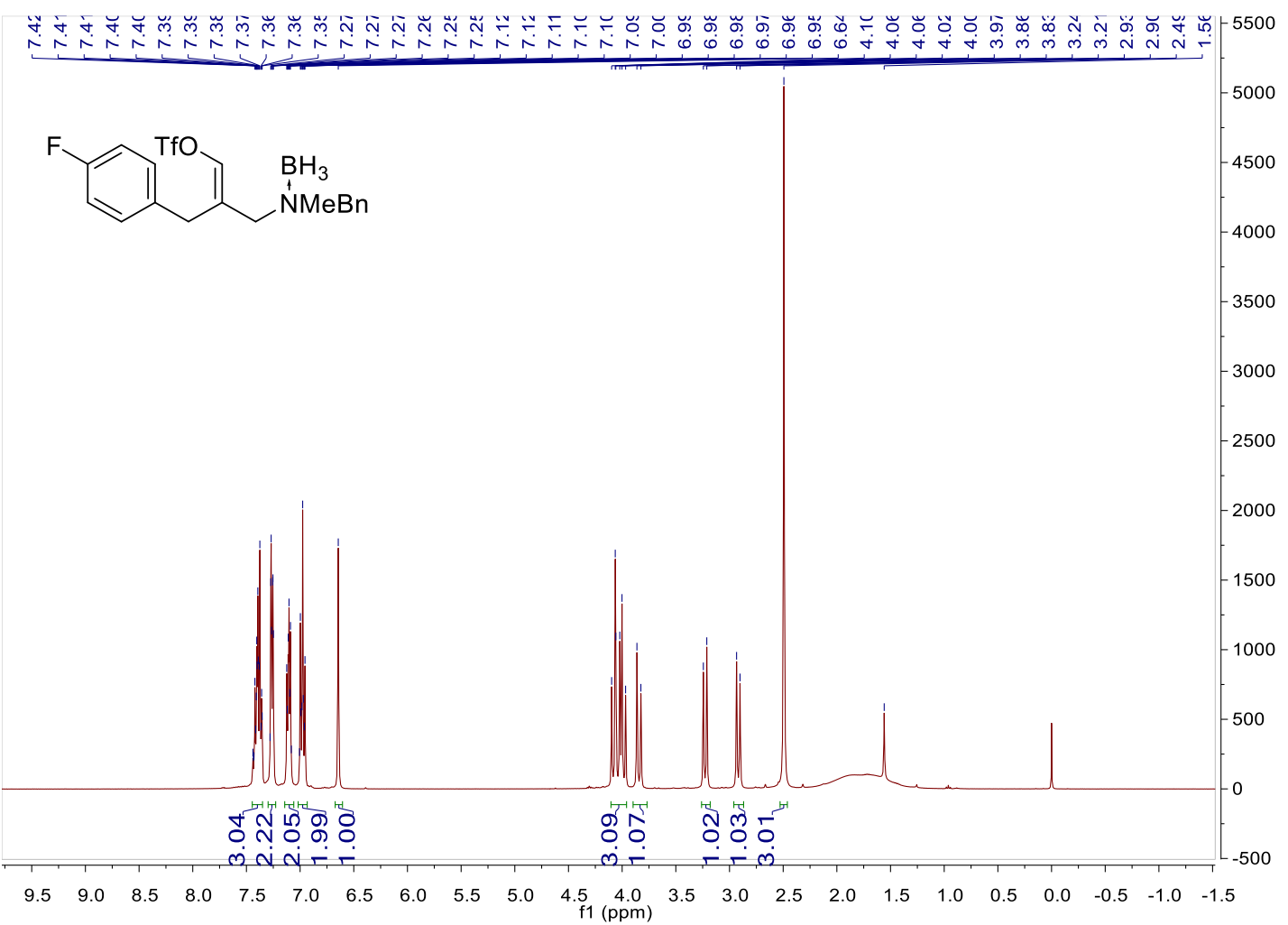

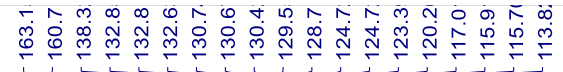

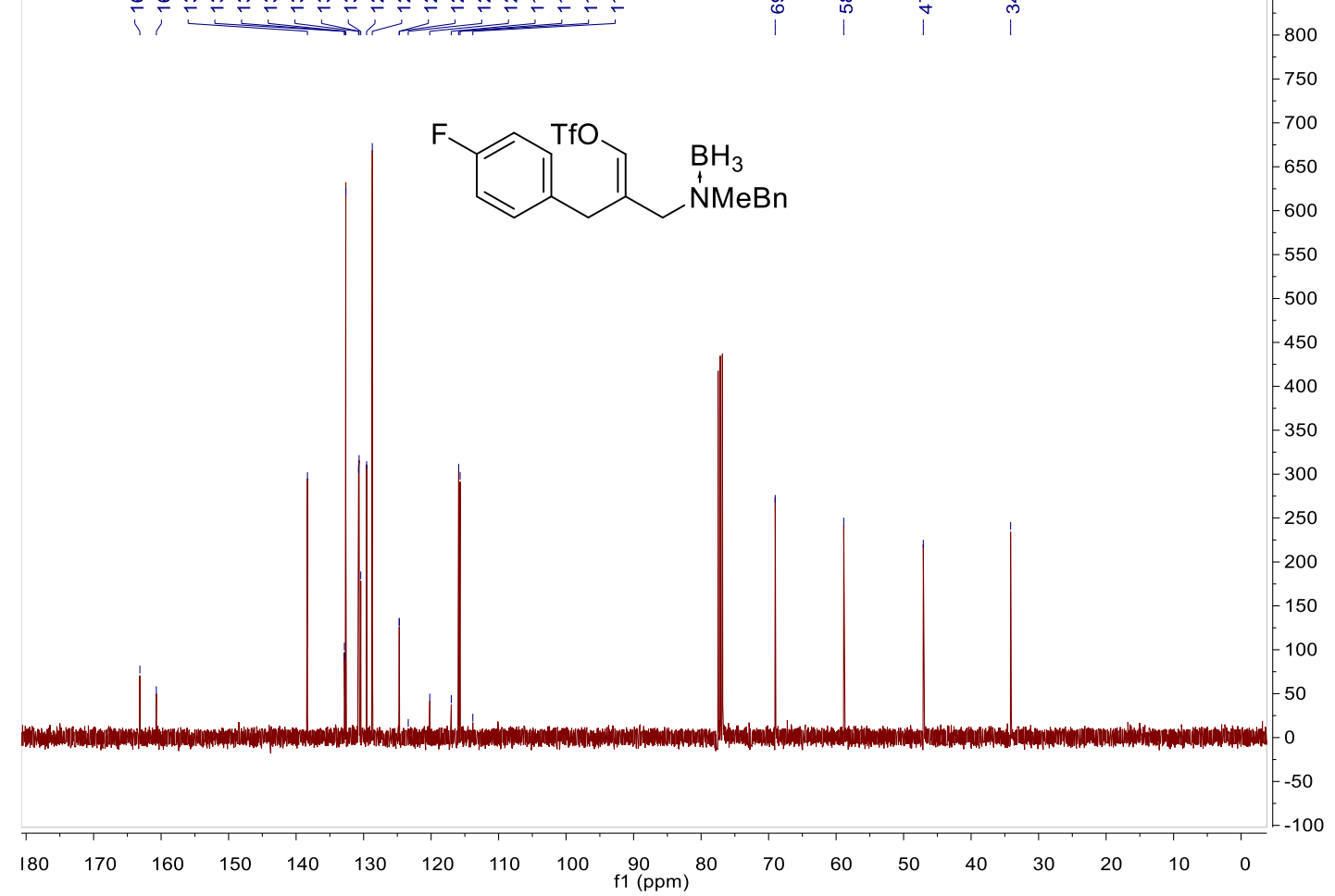



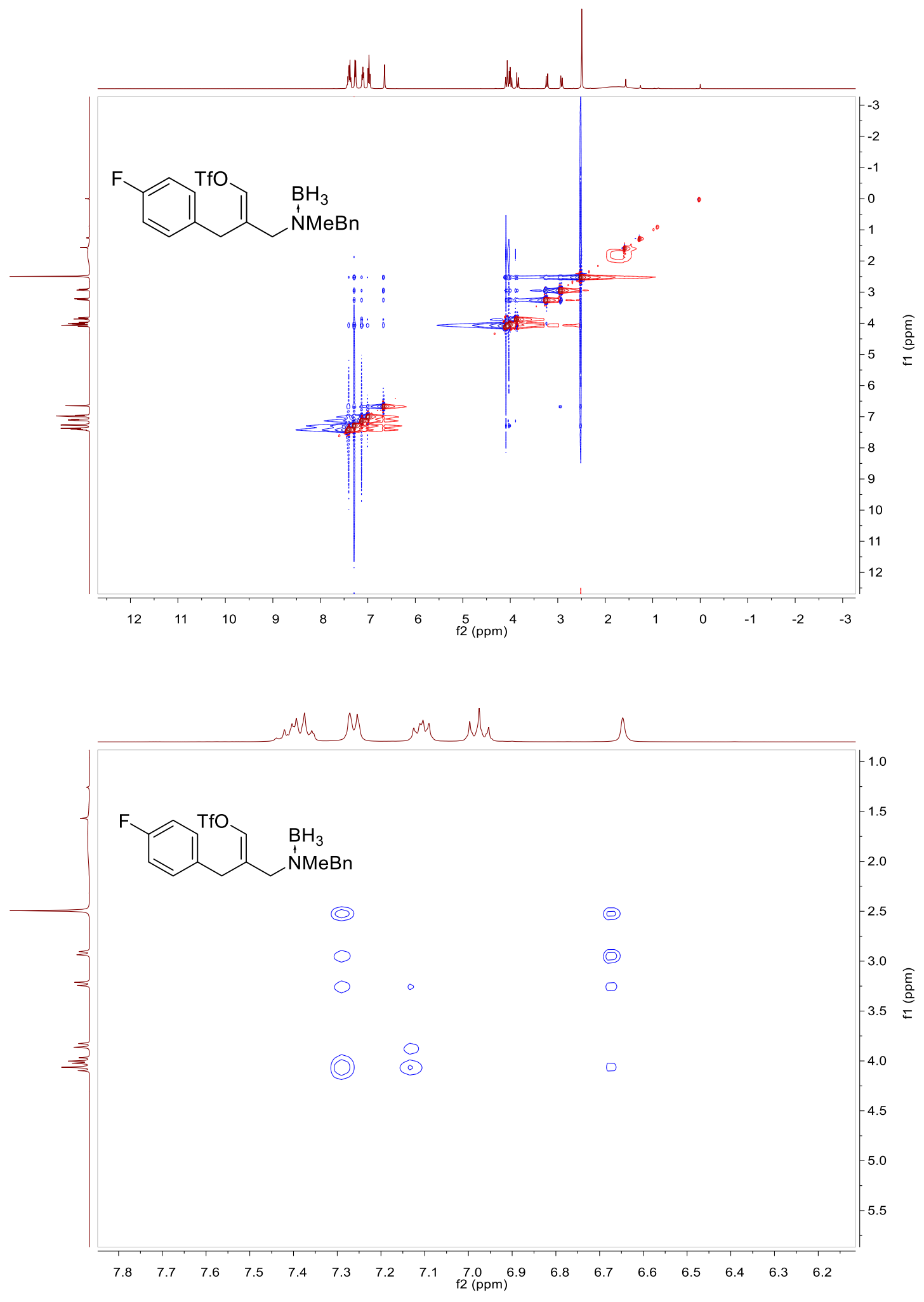
(Z)-3-(benzyl(methyl)aminoborane)-2-methylprop-1-en-1-yl

trifluoromethanesulfonate $(4 r)$

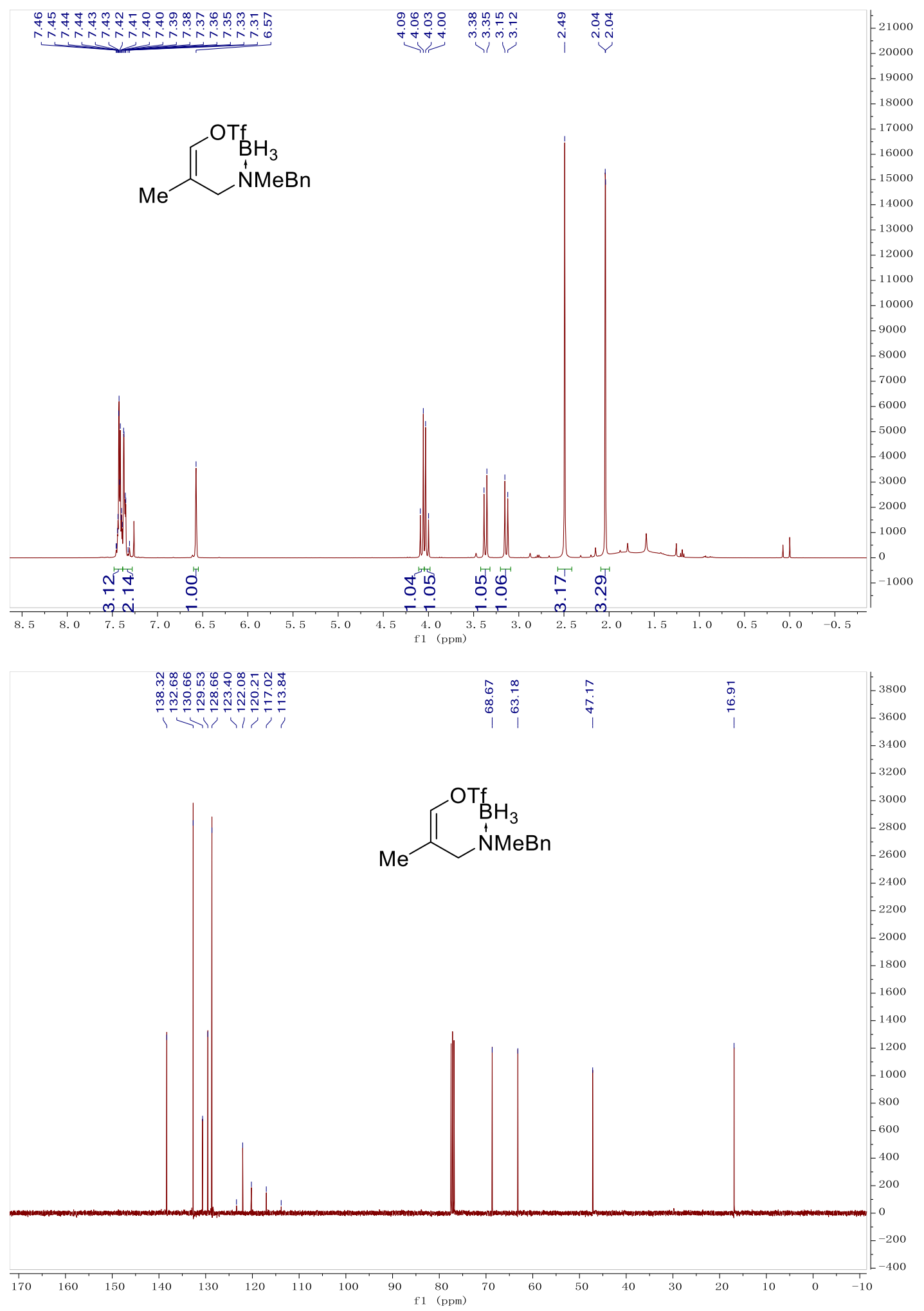




\section{(Z)-2-((benzyl(methyl)aminoborane)methyl)hex-1-en-1-yl}

\section{trifluoromethanesulfonate $(4 s)$}

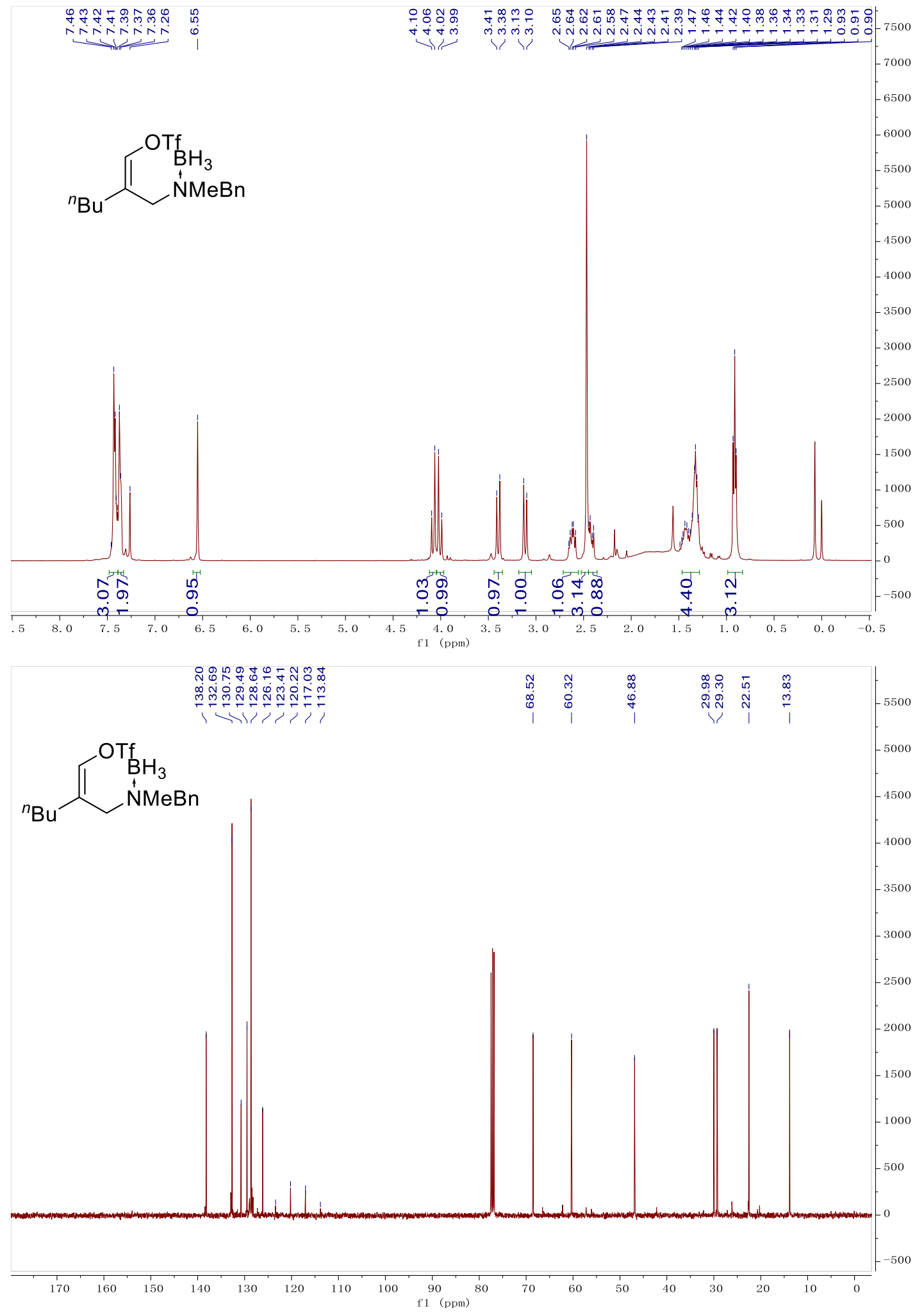


(Z)-2-((benzyl(methyl)aminoborane)methyl)-3-methylbut-1-en-1-yl

trifluoromethanesulfonate $(4 t)$

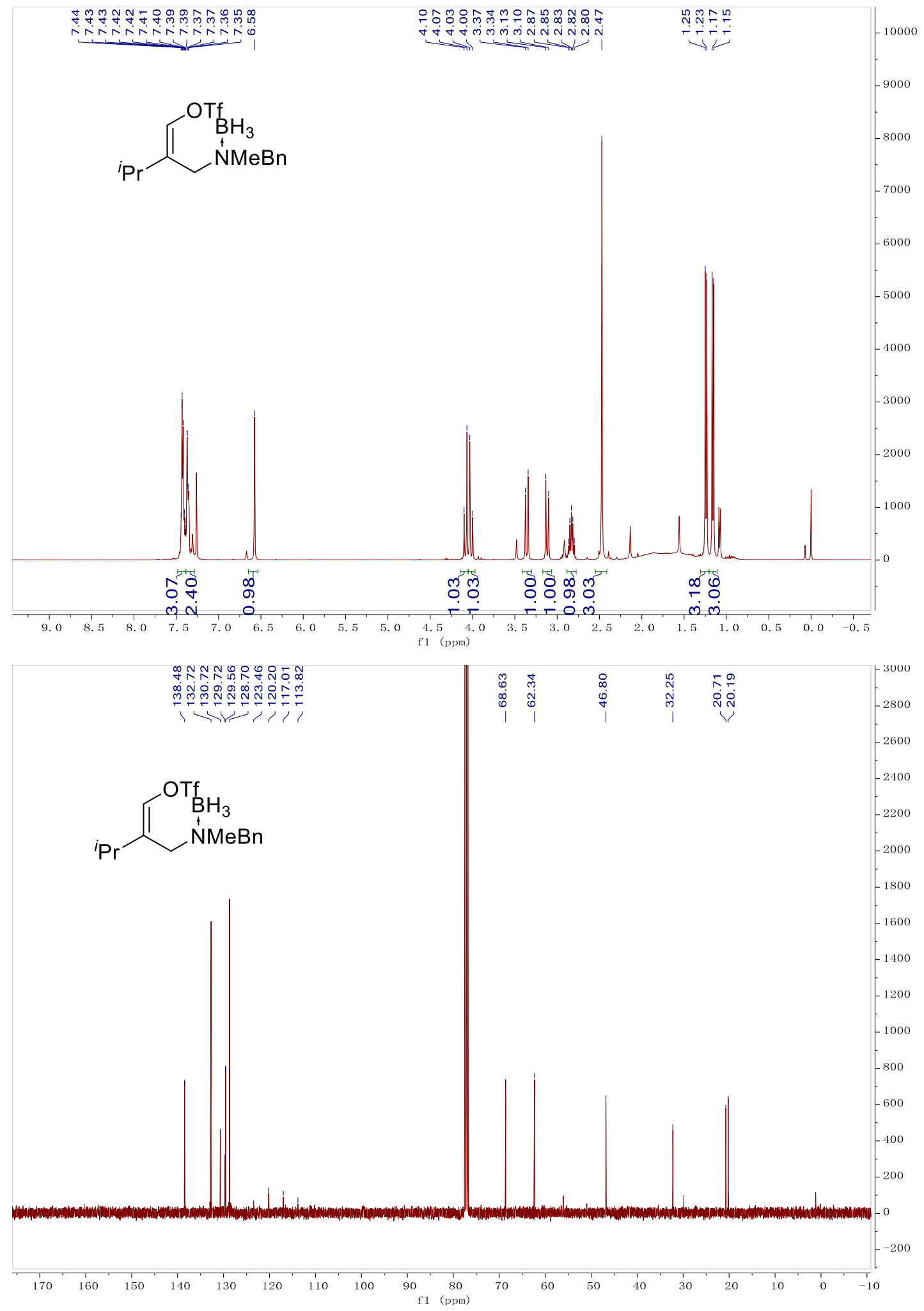




\section{(E)-3-(benzyl(methyl)aminoborane)-2-cyclohexylprop-1-en-1-yl}

\section{trifluoromethanesulfonate $(4 \mathrm{u})$}

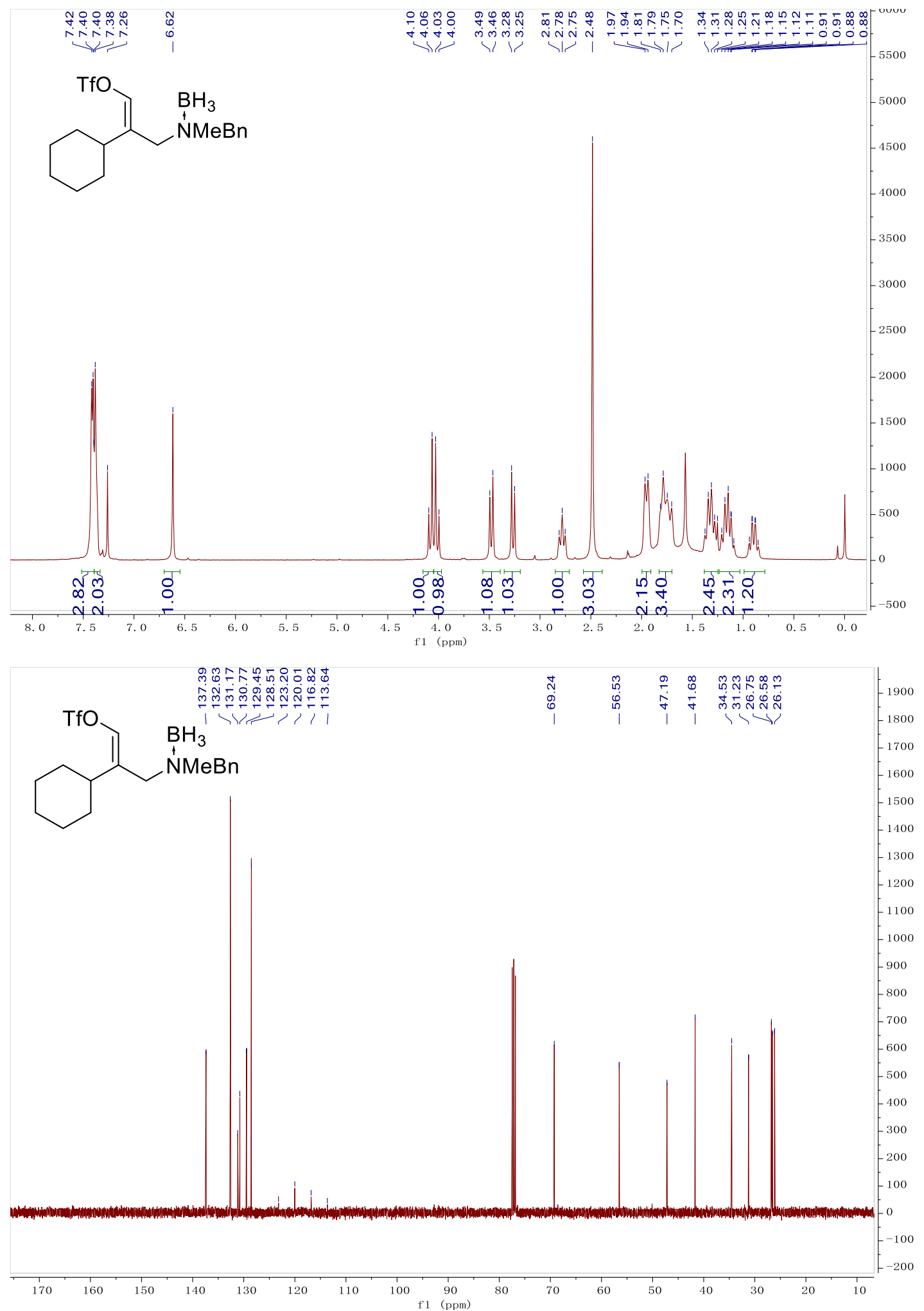


(E)-3-(dimethylaminoborane)-2-phenylprop-1-en-1-yl trifluoromethanesulfonate $(4 v)$

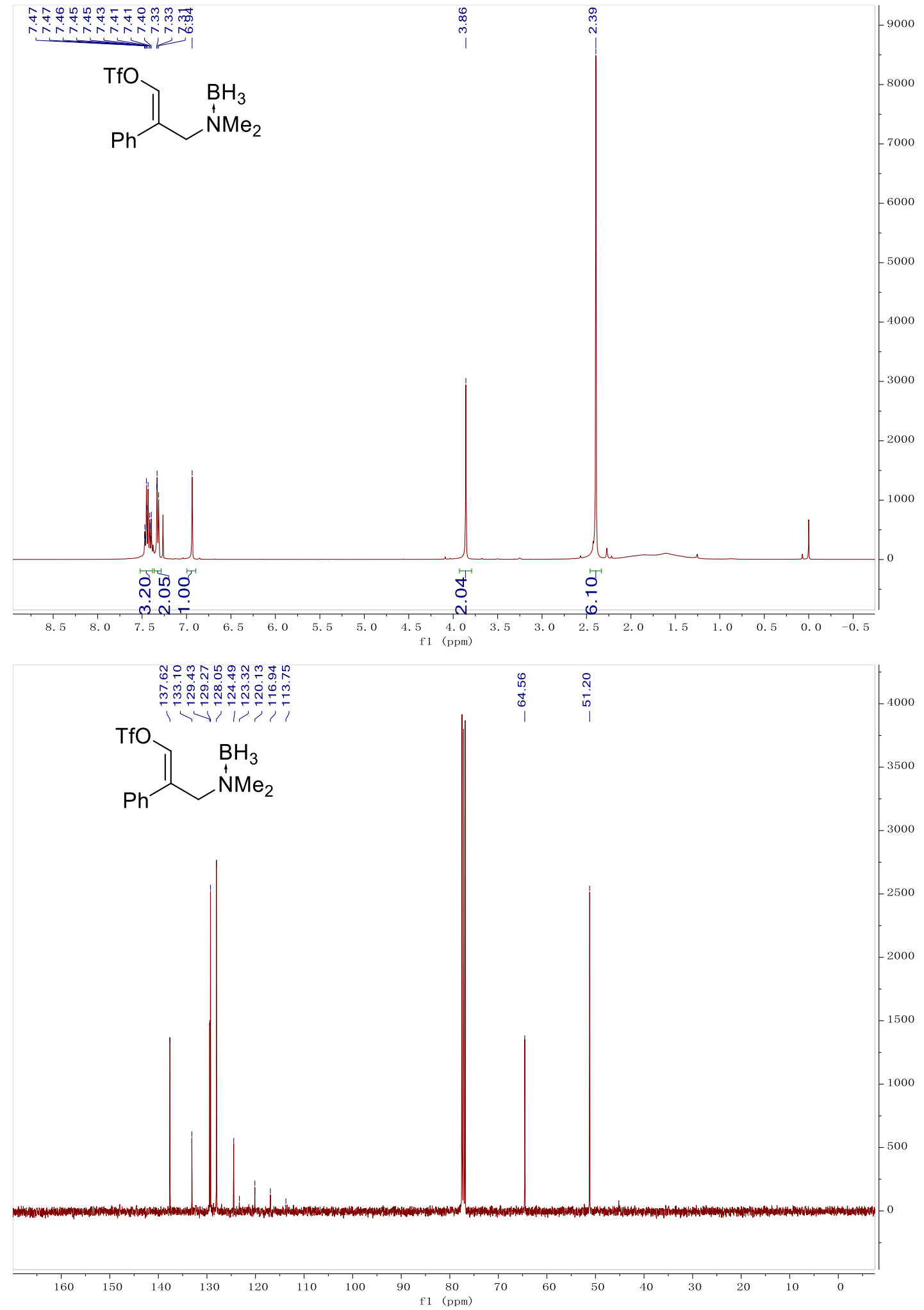




\section{(E)-3-(dimethylaminoborane)-2-(4-methoxyphenyl)prop-1-en-1-yl}

\section{trifluoromethanesulfonate $(4 w)$}

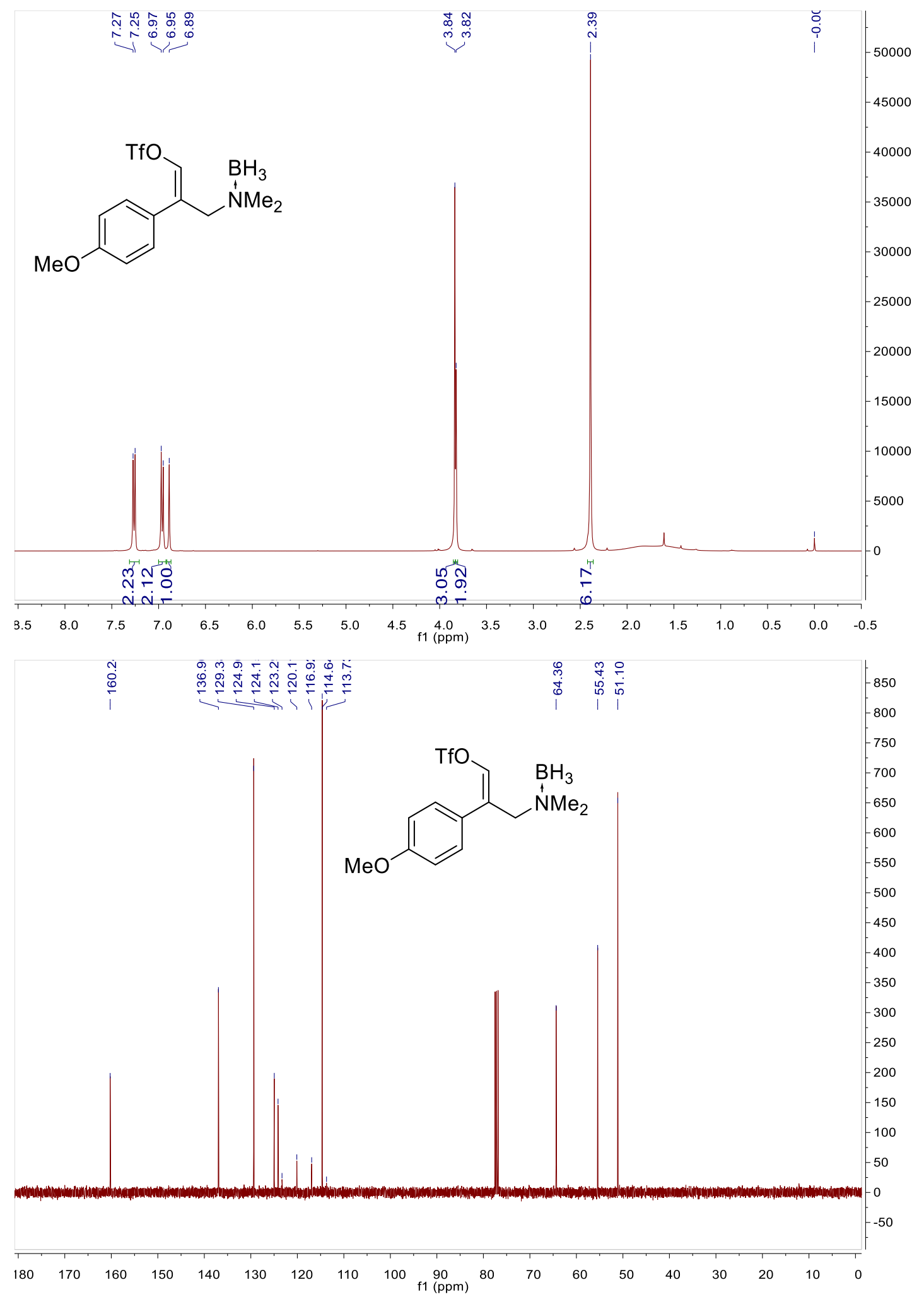




\section{(E)-2-(4-chlorophenyl)-3-(dimethylaminoborane)prop-1-en-1-yl}

\section{trifluoromethanesulfonate $(4 x)$}
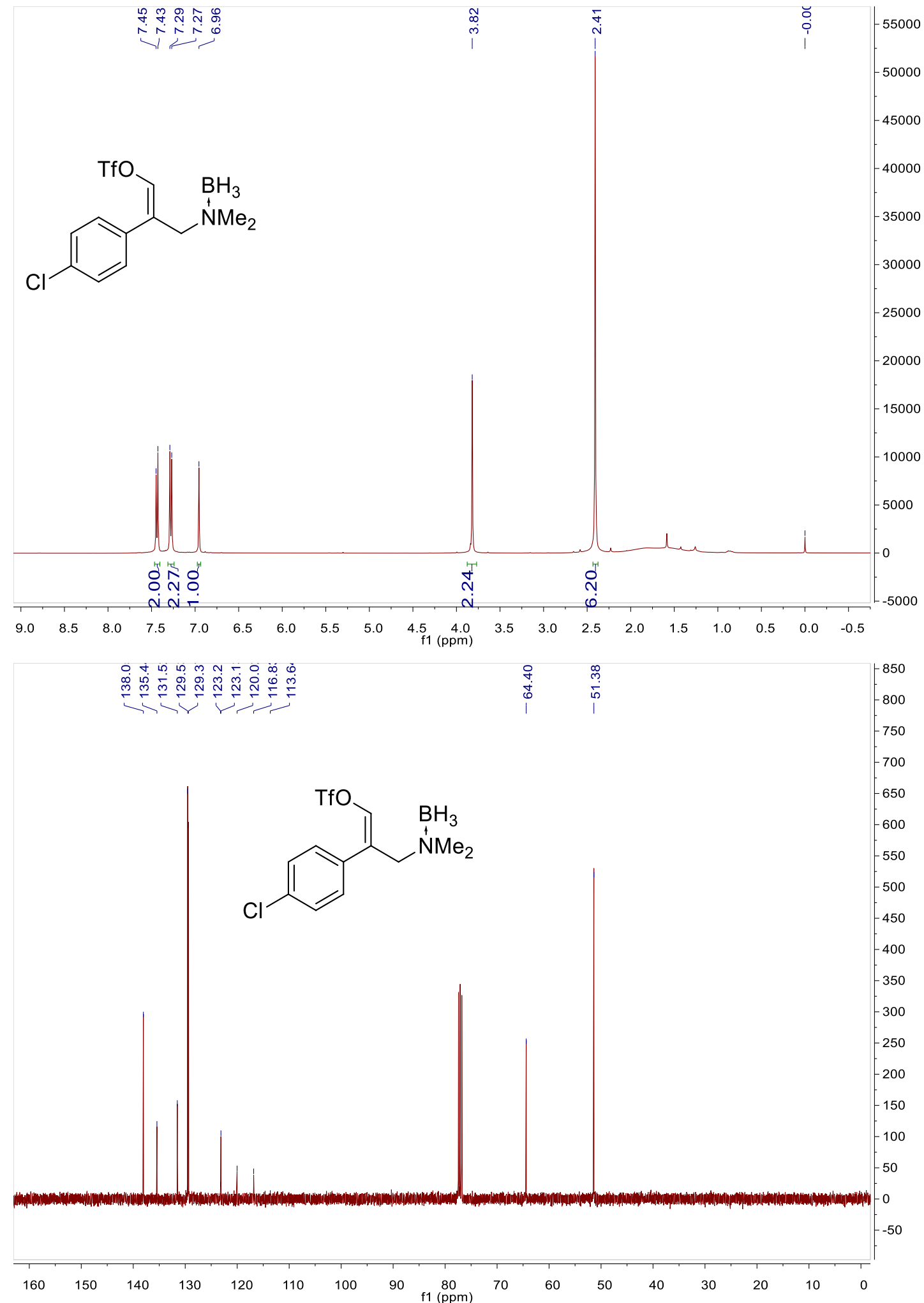
(Z)-3-(dimethylaminoborane)-2-(4-(trifluoromethyl)phenyl)prop-1-en-1-yl

trifluoromethanesulfonate $(4 y)$
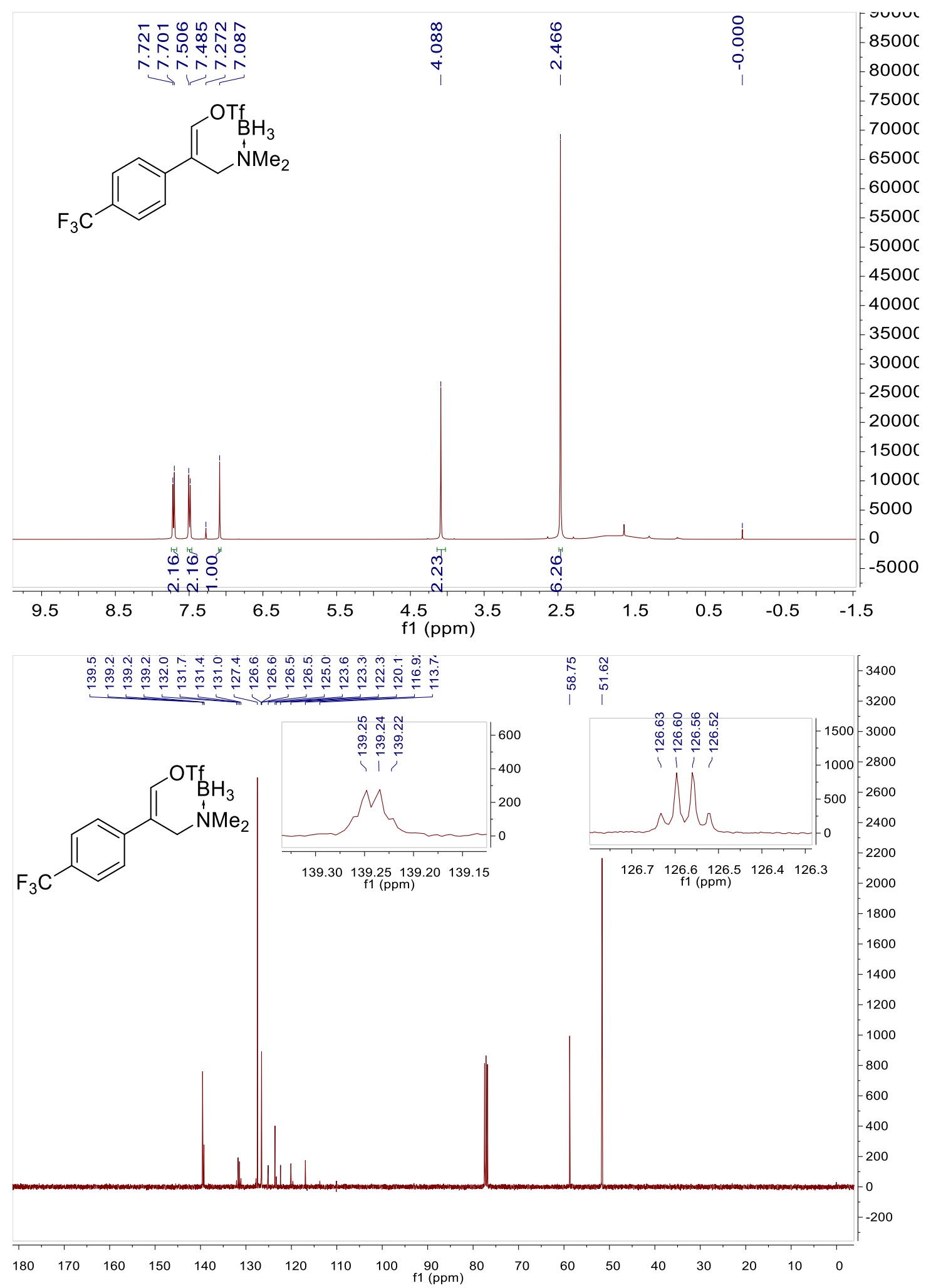


\section{(4-(trimethylamine-boranylmethylene)cyclohexyl)benzene (3aa)}

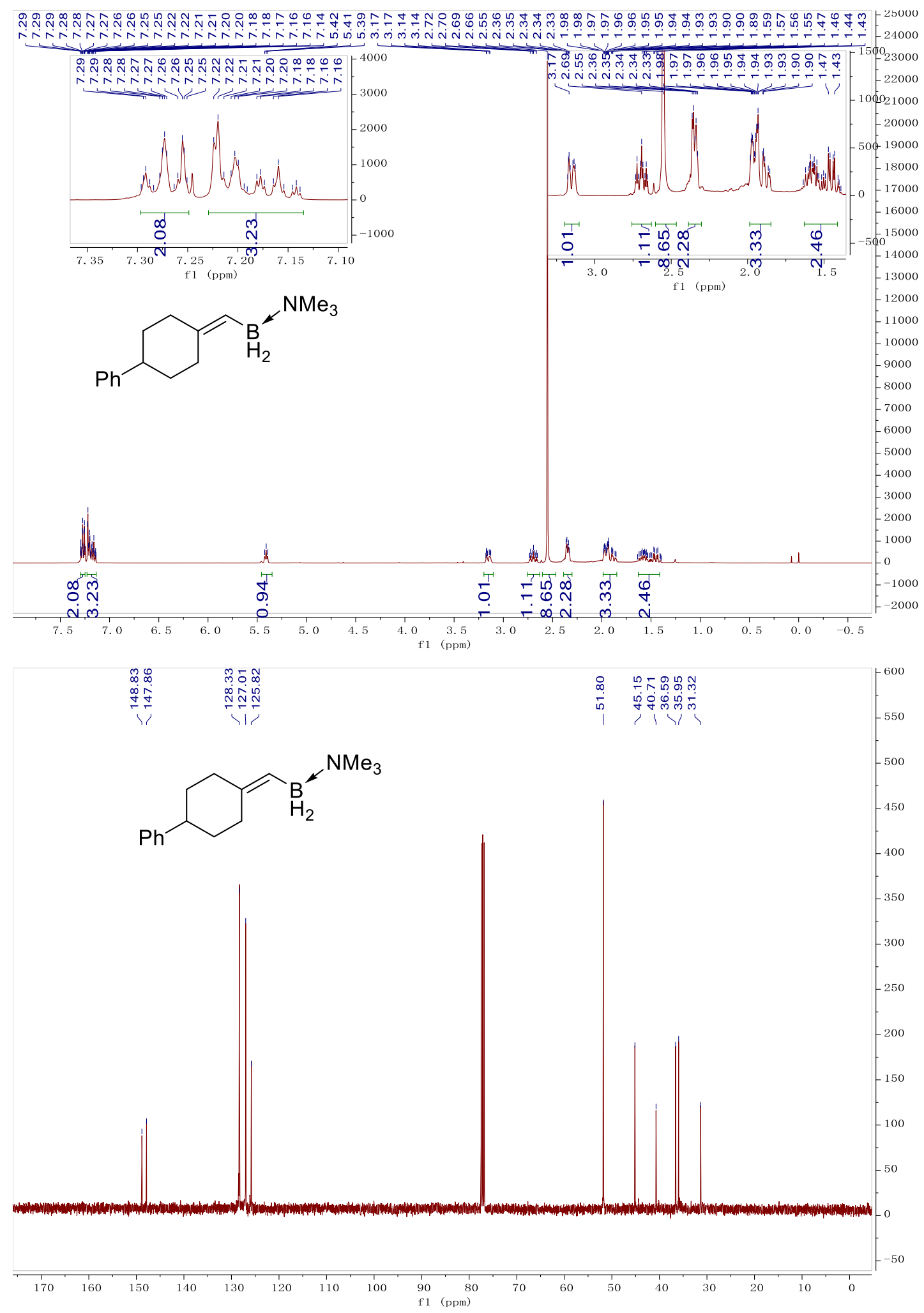


(4-(tributylphosphane-boranylmethylene)cyclohexyl)benzene (3ab)

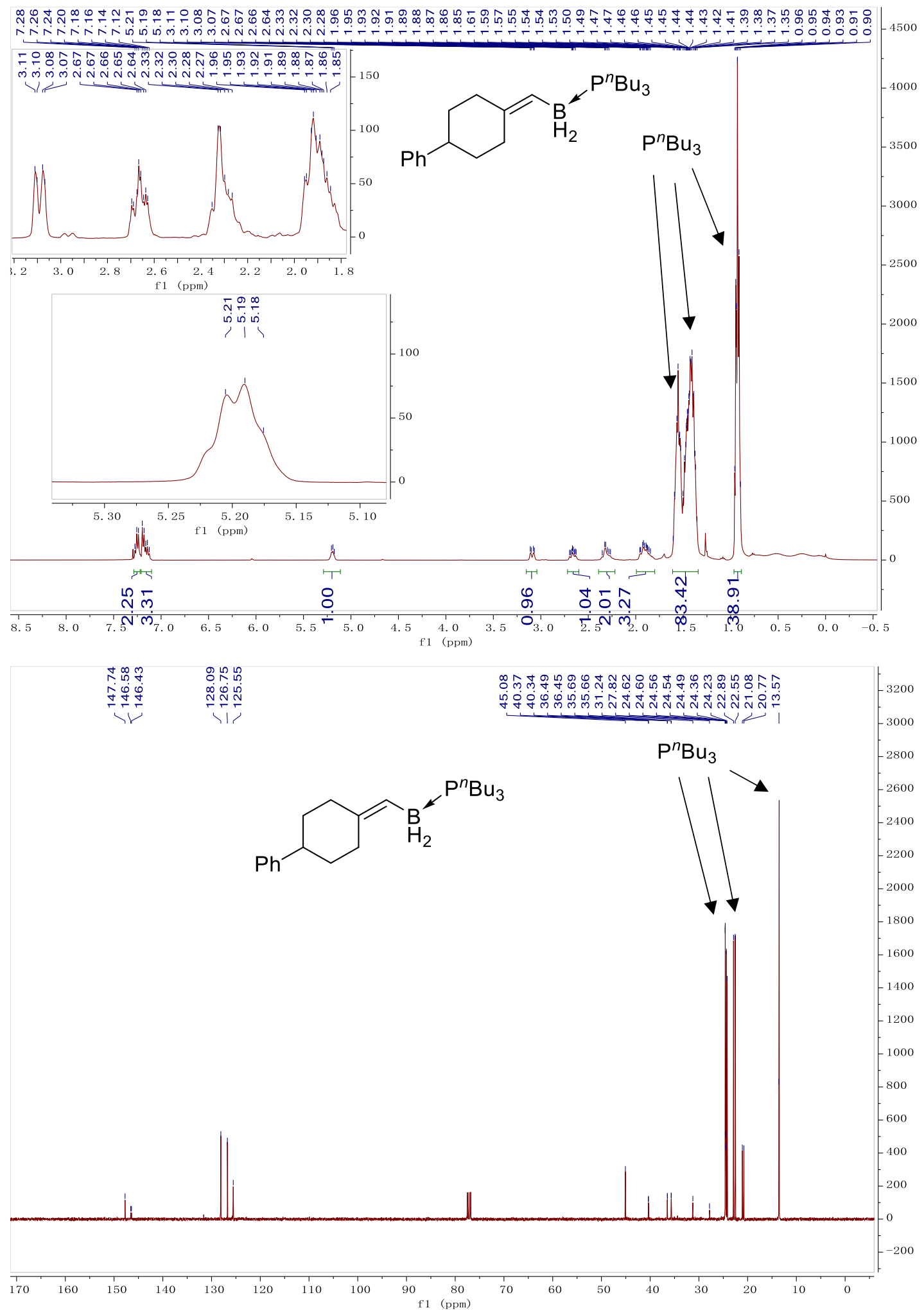




\section{(1,3-dimethyl-1H-imidazol-3-ium-2-yl)((4-}

phenylcyclohexylidene)methyl)dihydroborate (3ac)

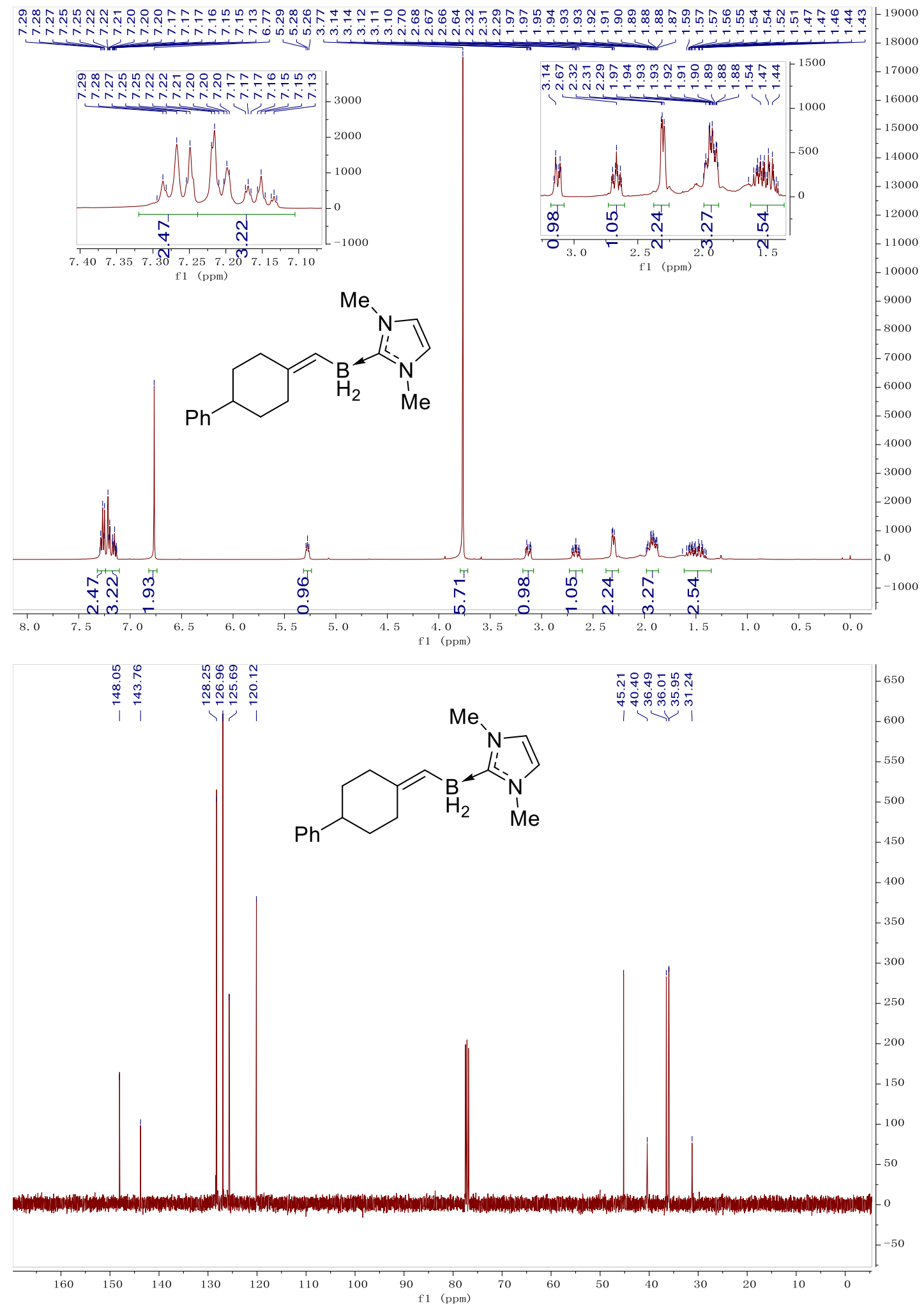


(trimethylamine-boranylmethylene)cyclopentane (3ba)

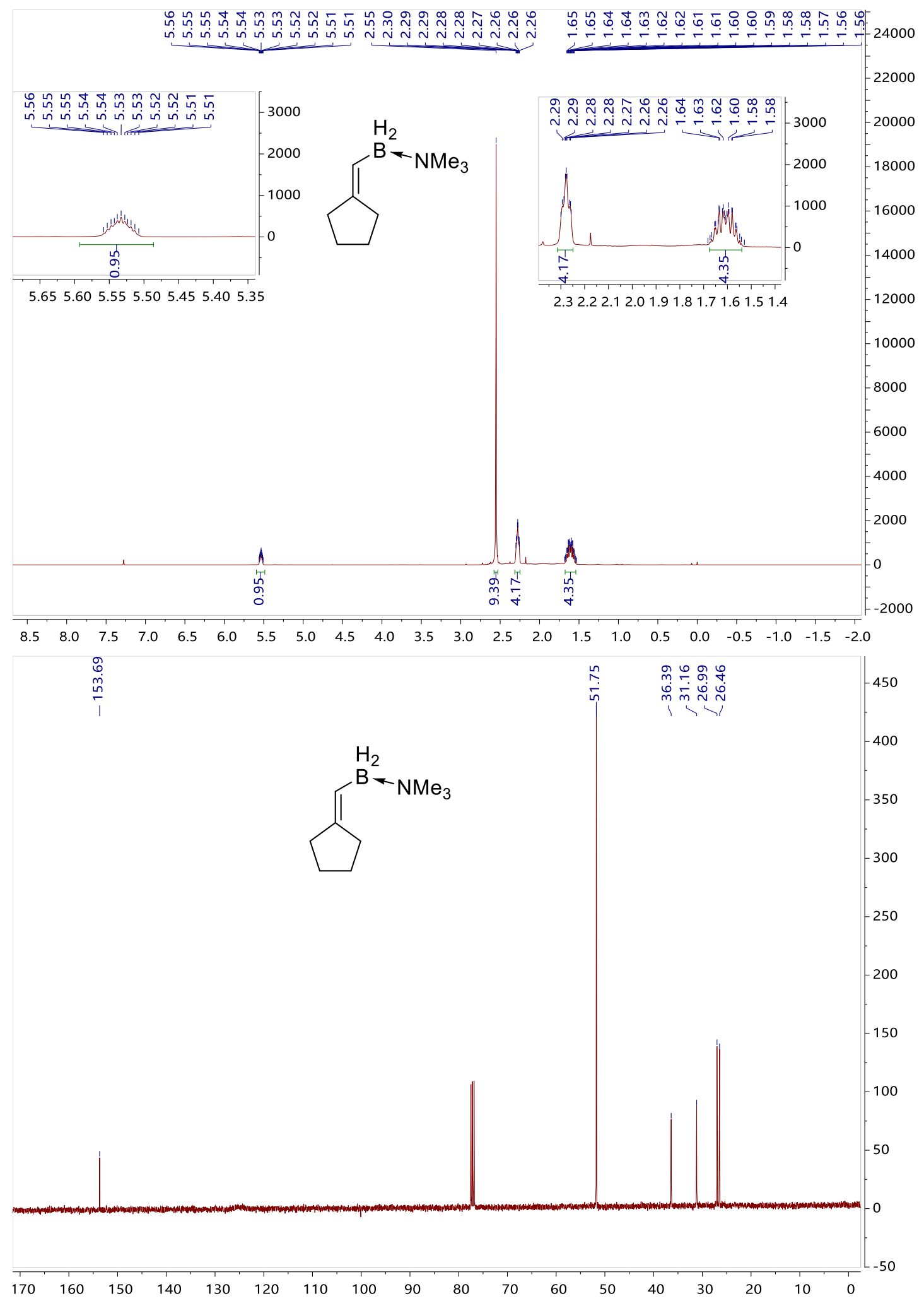


(trimethylamine-boranylmethylene)cyclohexane (3ca)

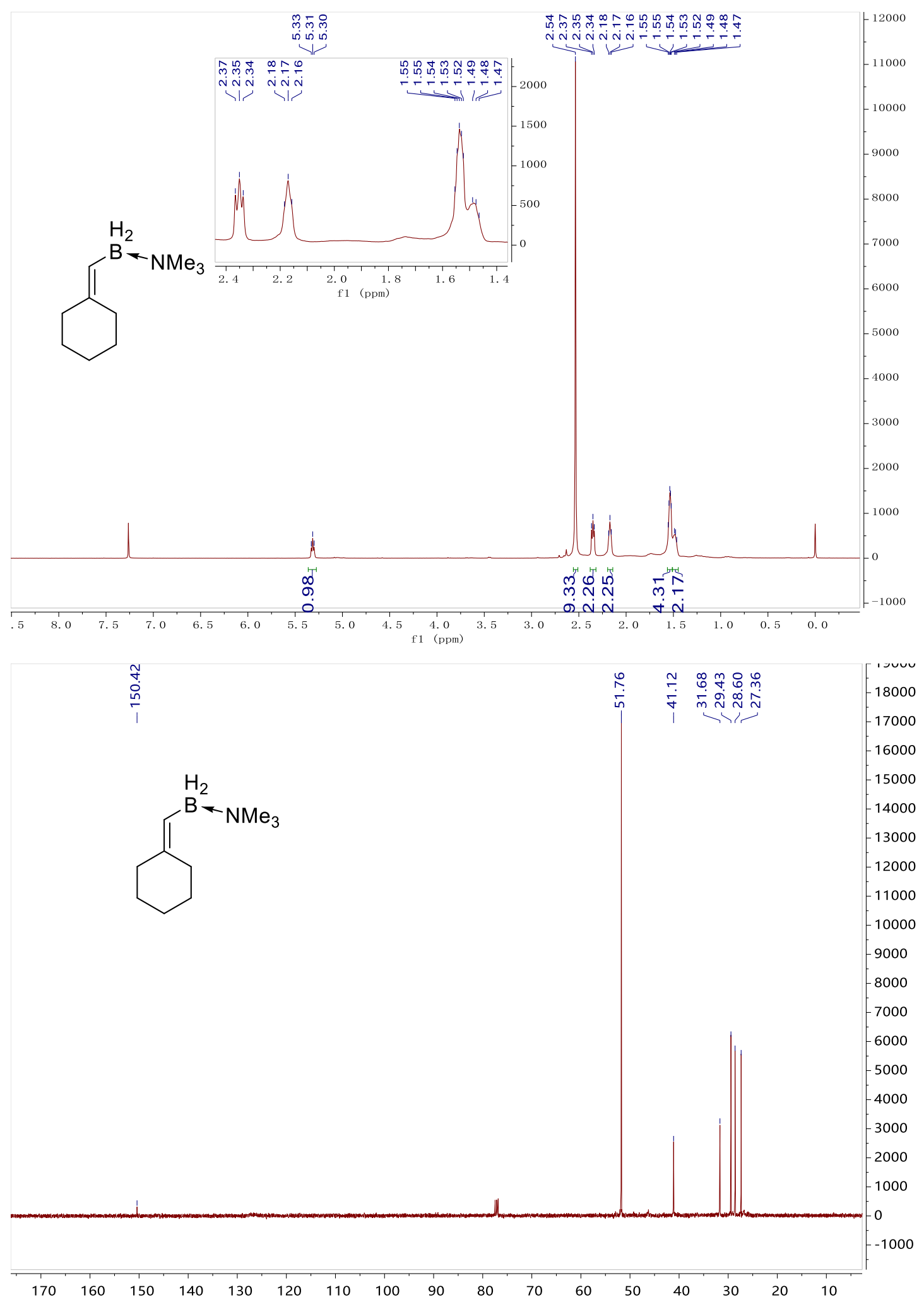


(trimethylamine-boranylmethylene) cycloheptane (3da)

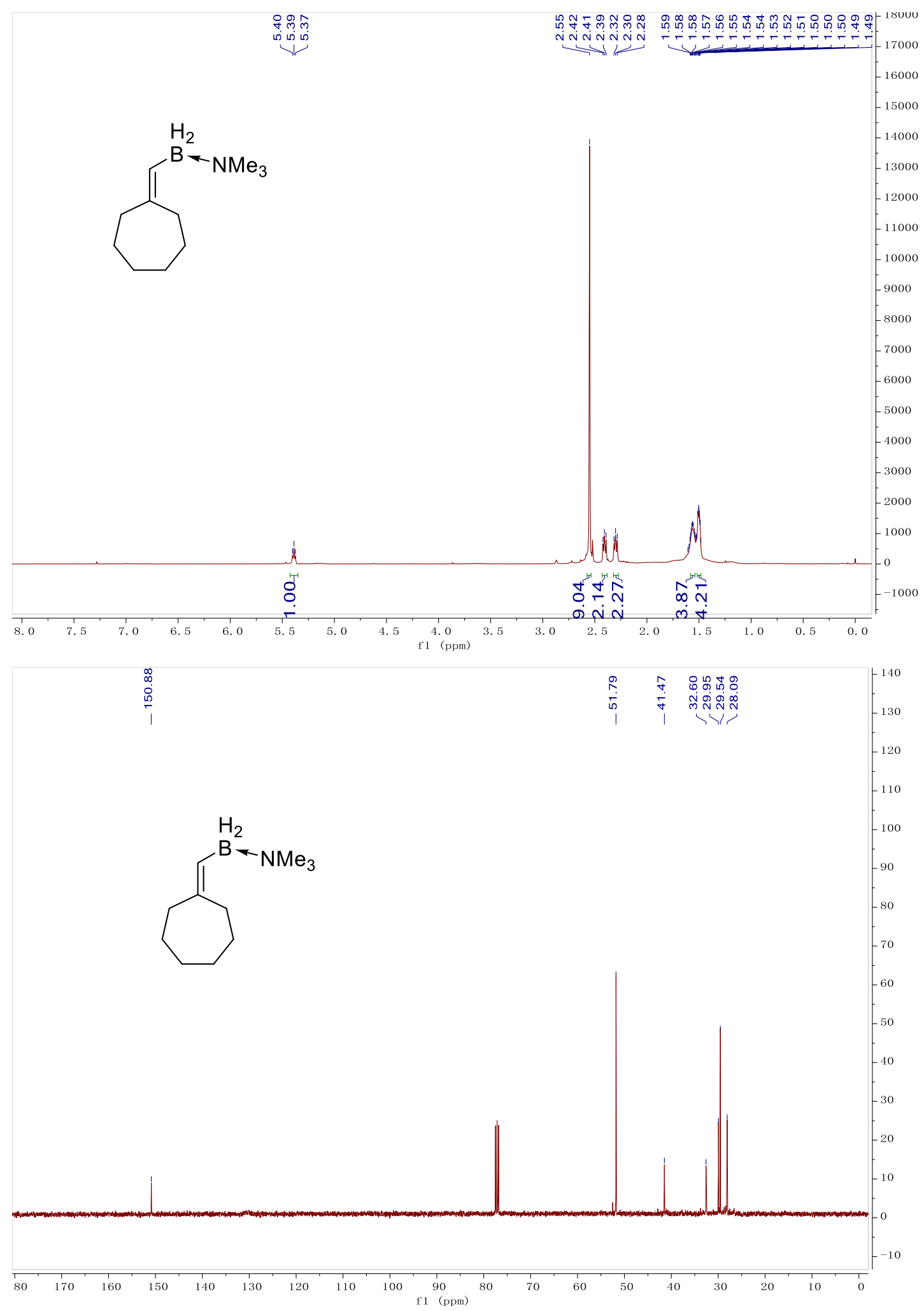


1-(trimethylamine-boranyl)-2-methylprop-1-ene (3ea)

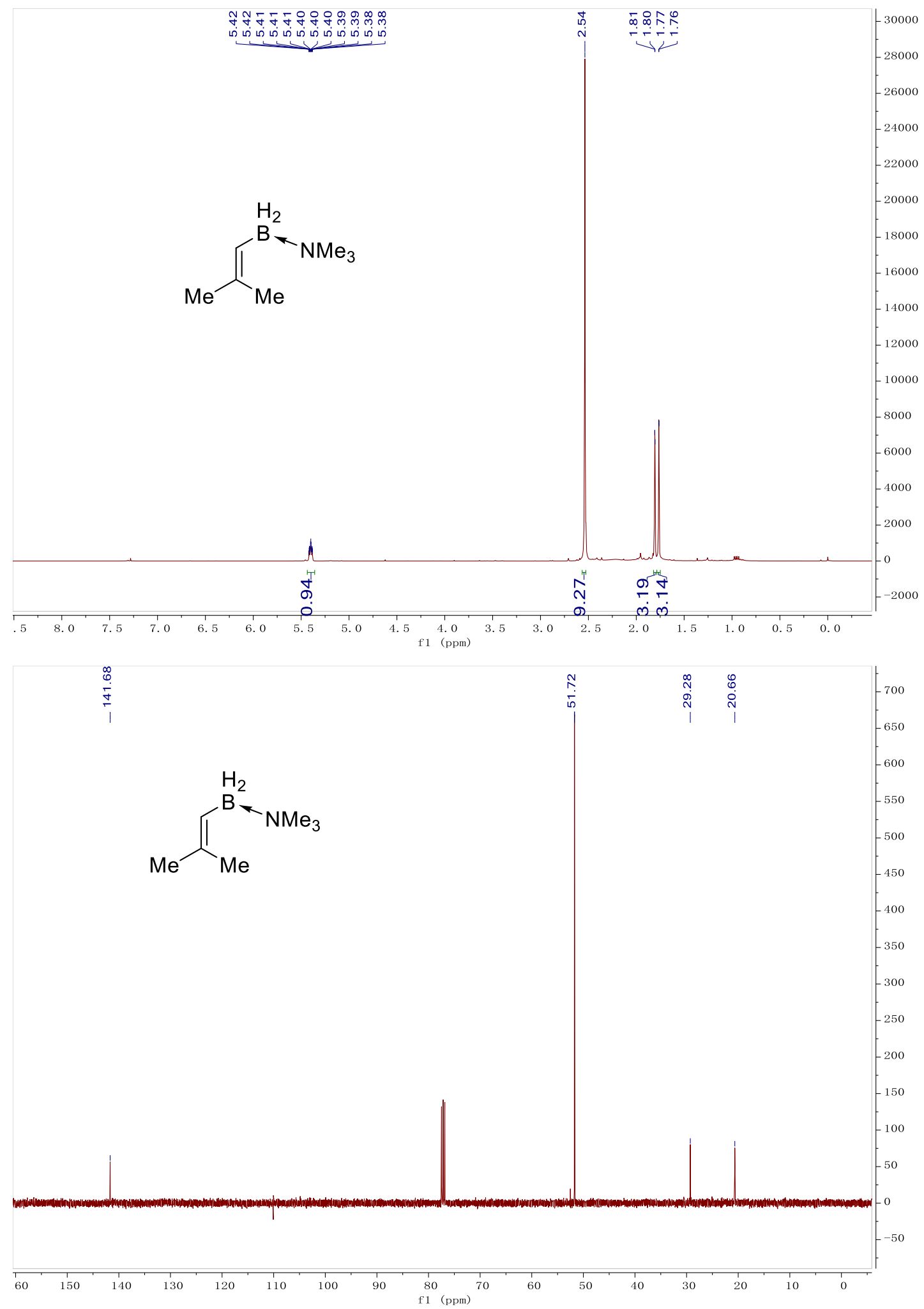




\section{3-(trimethylamine-boranylmethylene)pentane (3fa)}

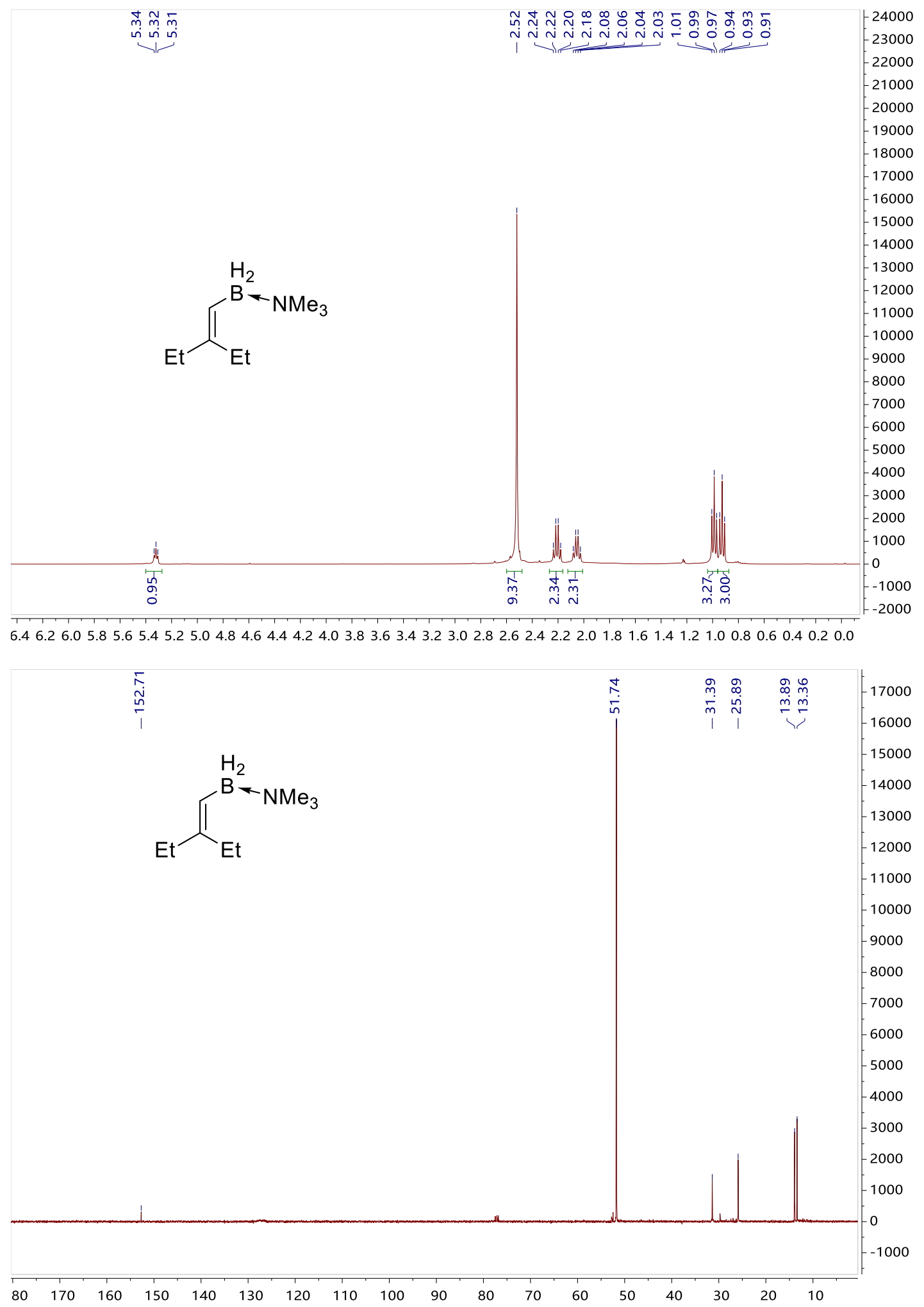


7-(trimethylamine-boranylmethylene)tridecane (3ga)

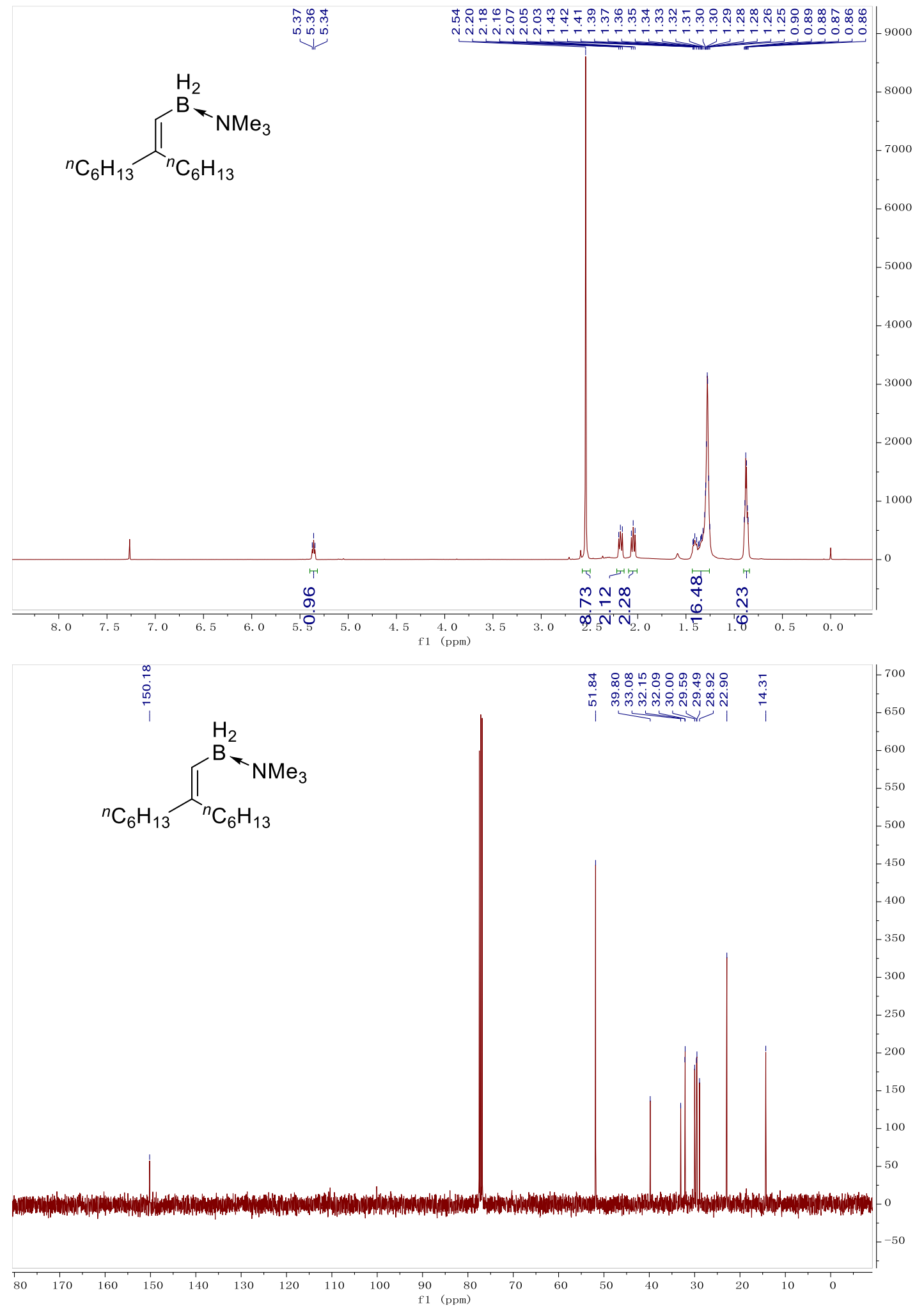


1-(3-(trimethylamine-boranyl)-2-methylallyl)-4-(tert-butyl)benzene (3ha)

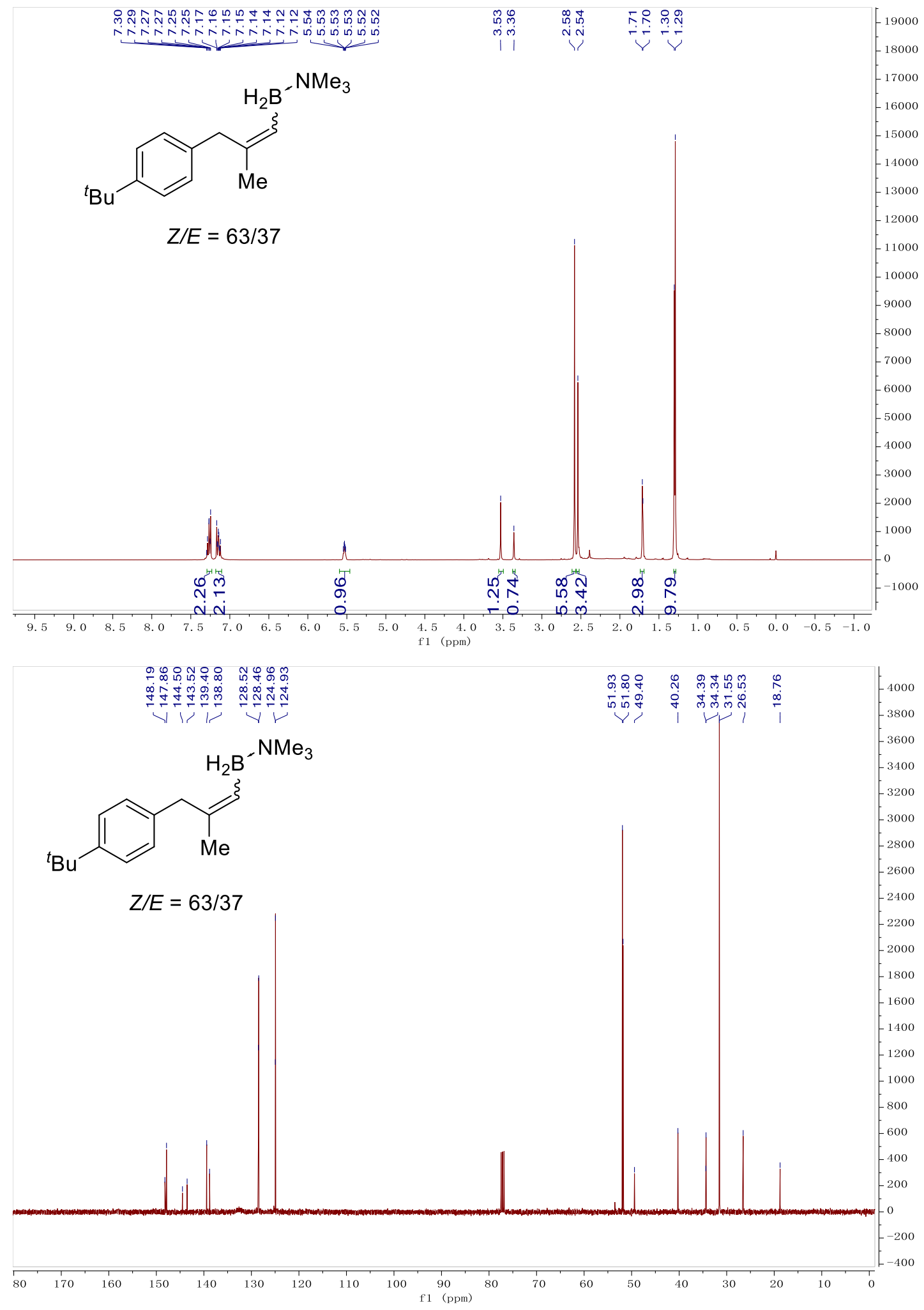


4-benzyl-1,1-dimethyl-2,5-dihydro-1 $H$-1 $\lambda^{4}, 2$-azaborole (5a)

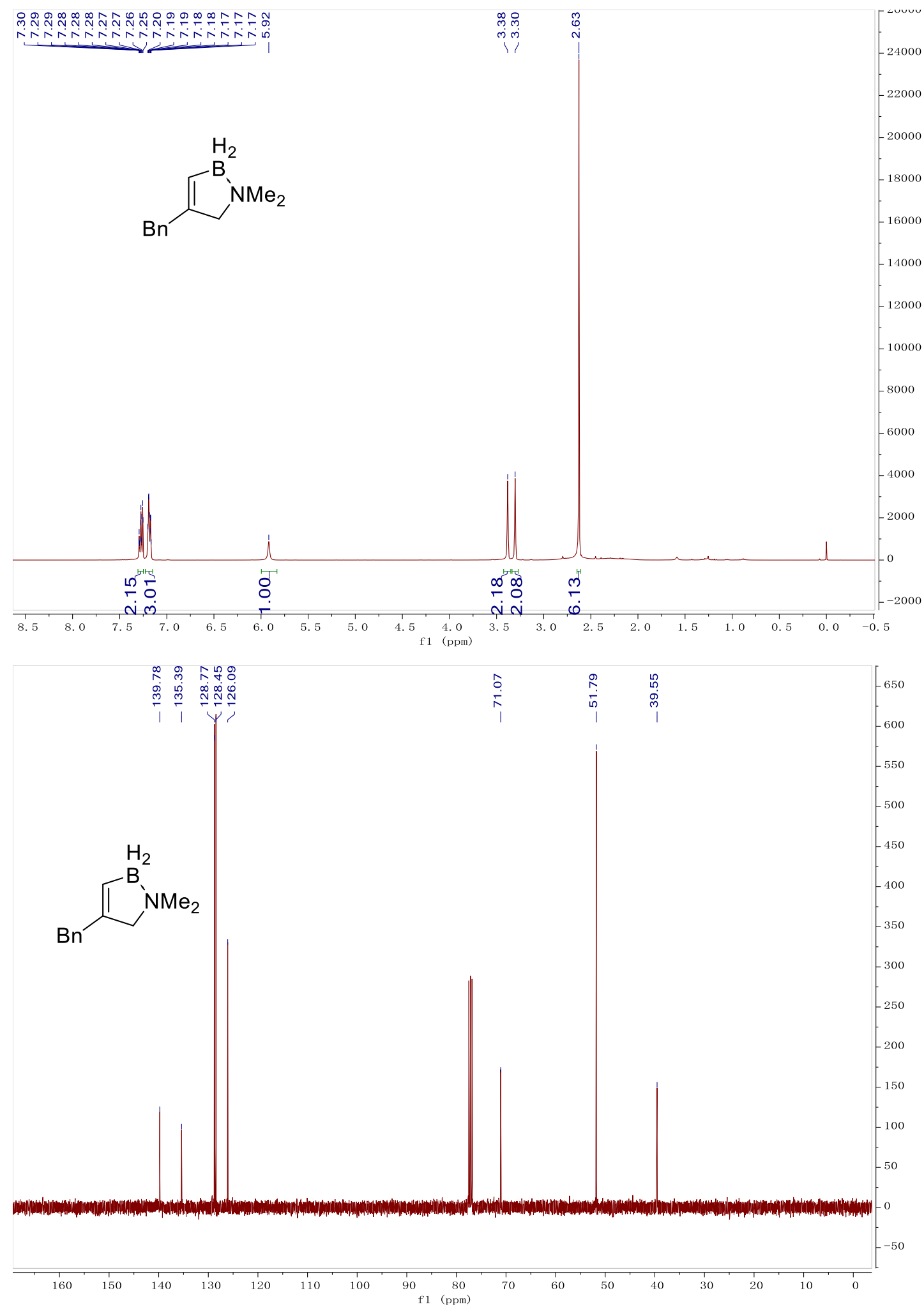




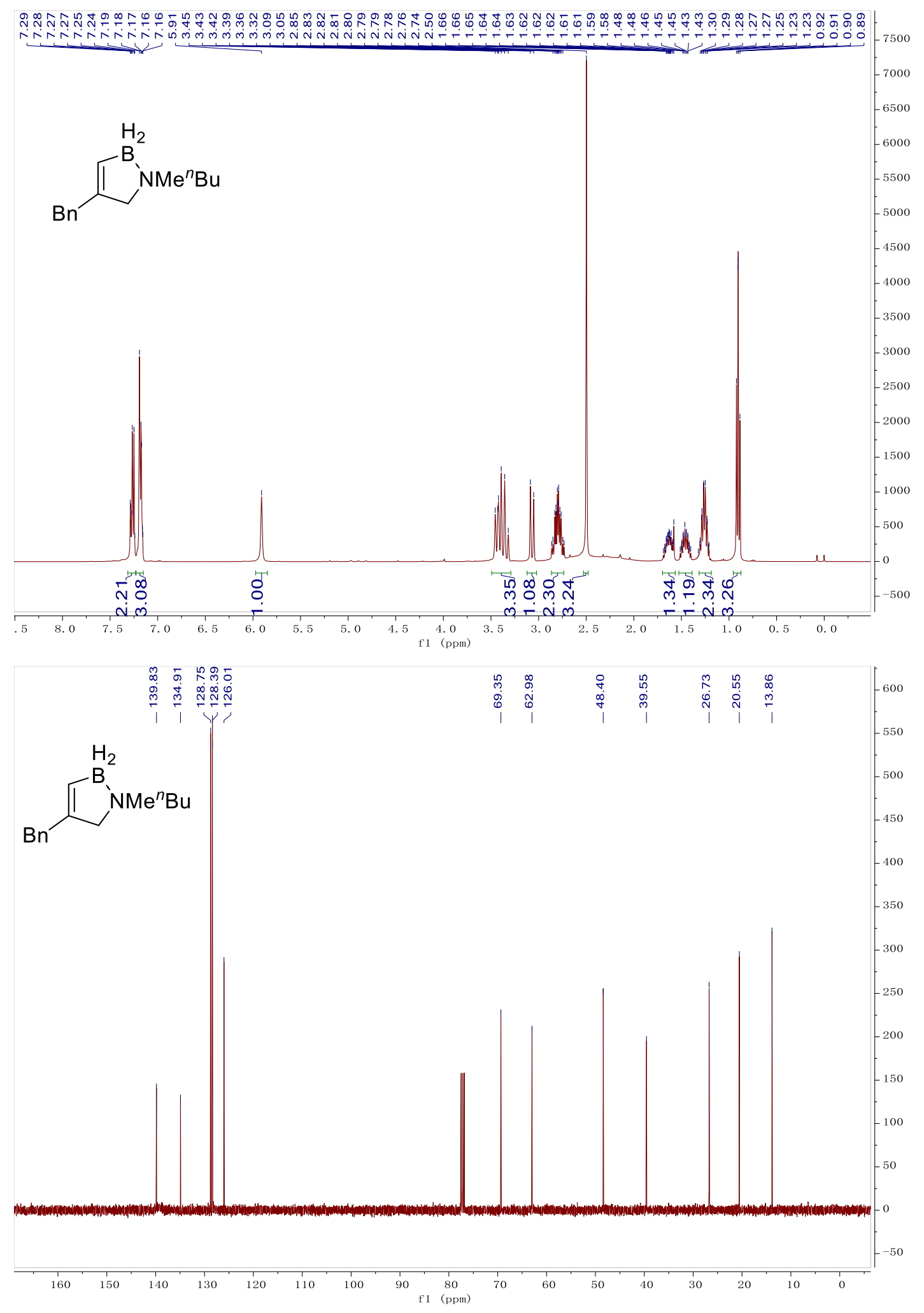


1,4-dibenzyl-1-methyl-2,5-dihydro- $1 H$-1 $\lambda^{4}, 2$-azaborole (5c)

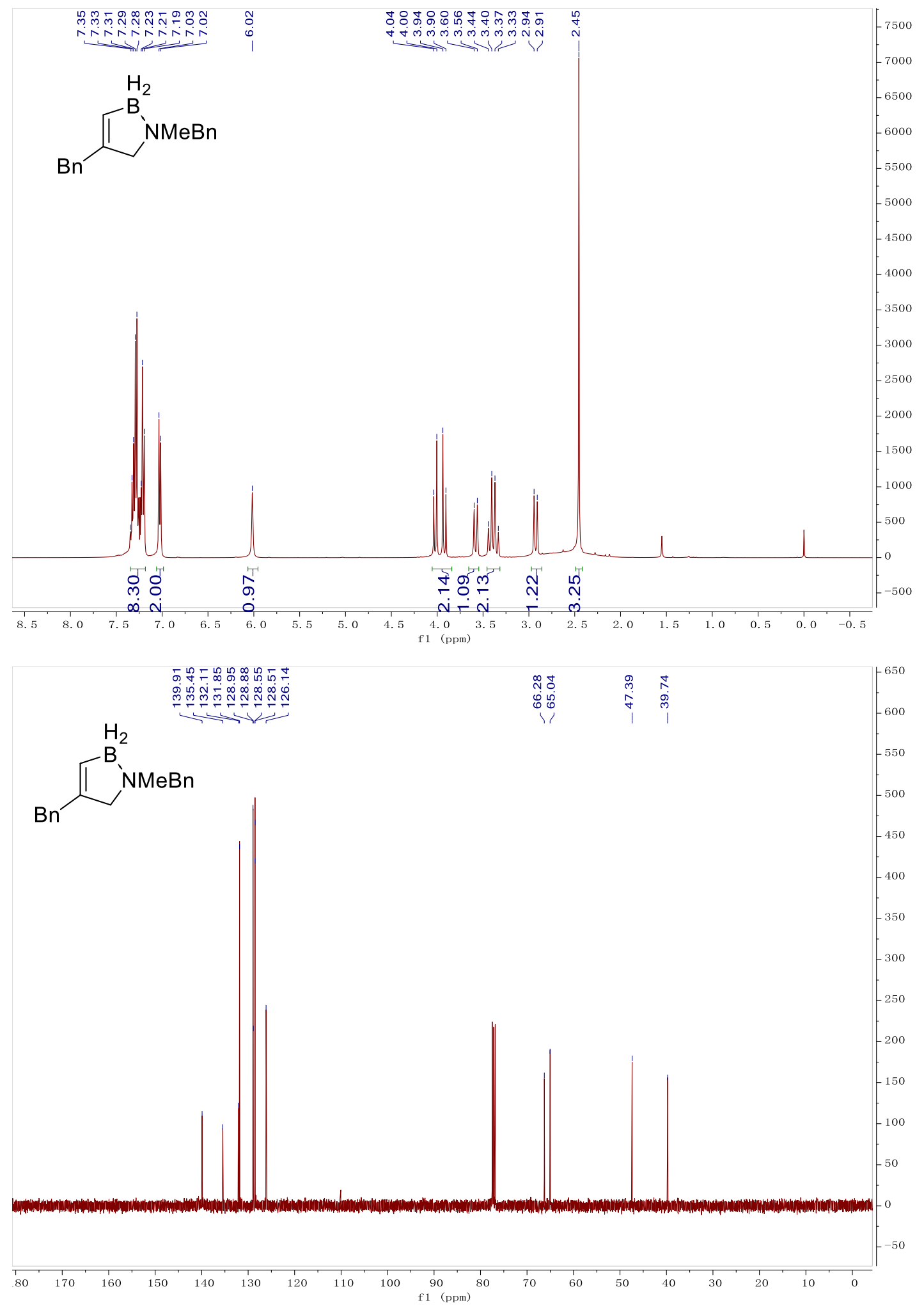


7-benzyl-4 $\lambda^{4}$-aza-5-boraspiro[3.4]oct-6-ene (5d)

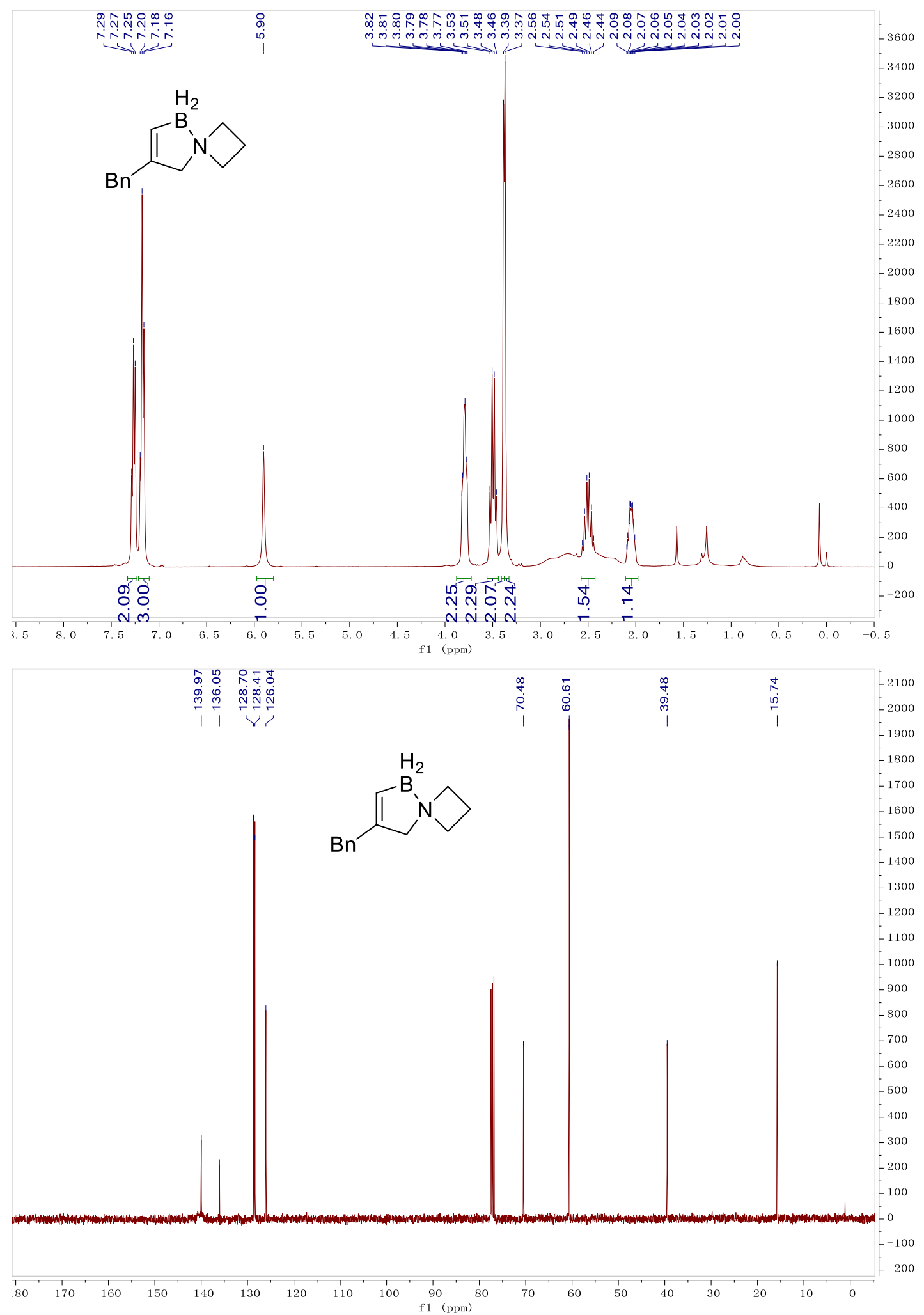




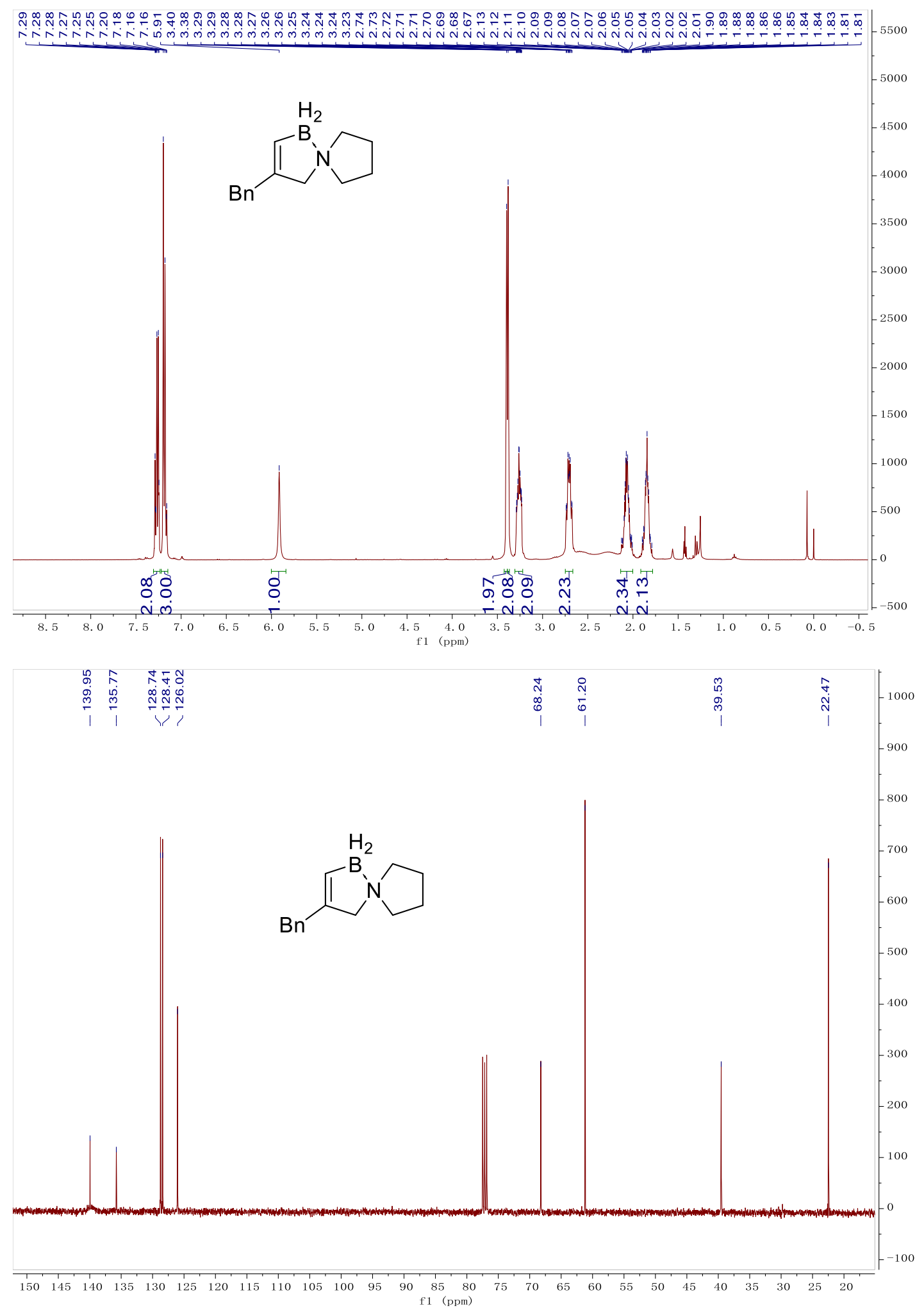


3-benzyl-5 $\lambda^{4}$-aza-1-boraspiro[4.5]dec-2-ene (5f)

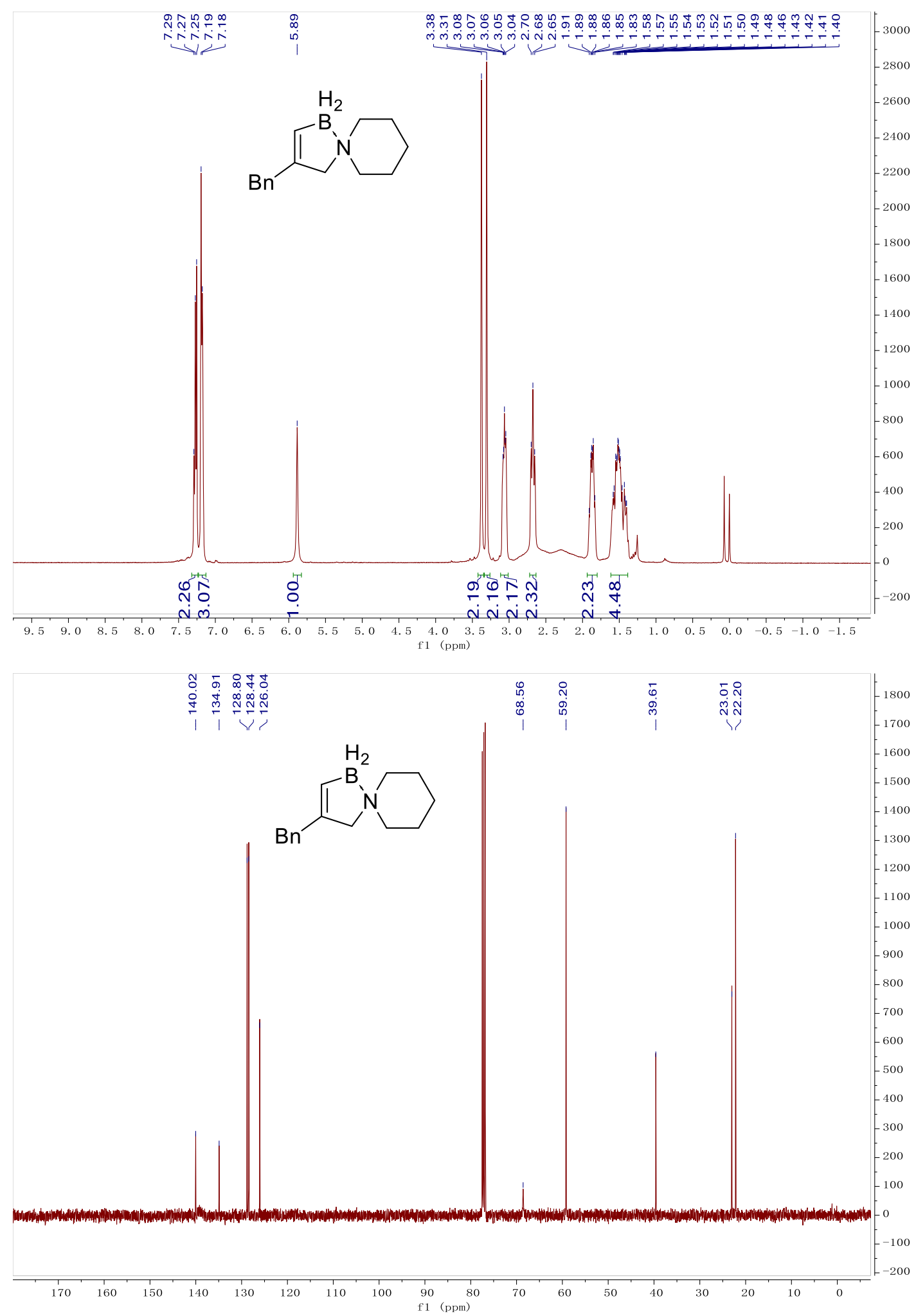


3-benzyl-5 $\lambda^{4}$-aza-1-boraspiro[4.6]undec-2-ene (5g)

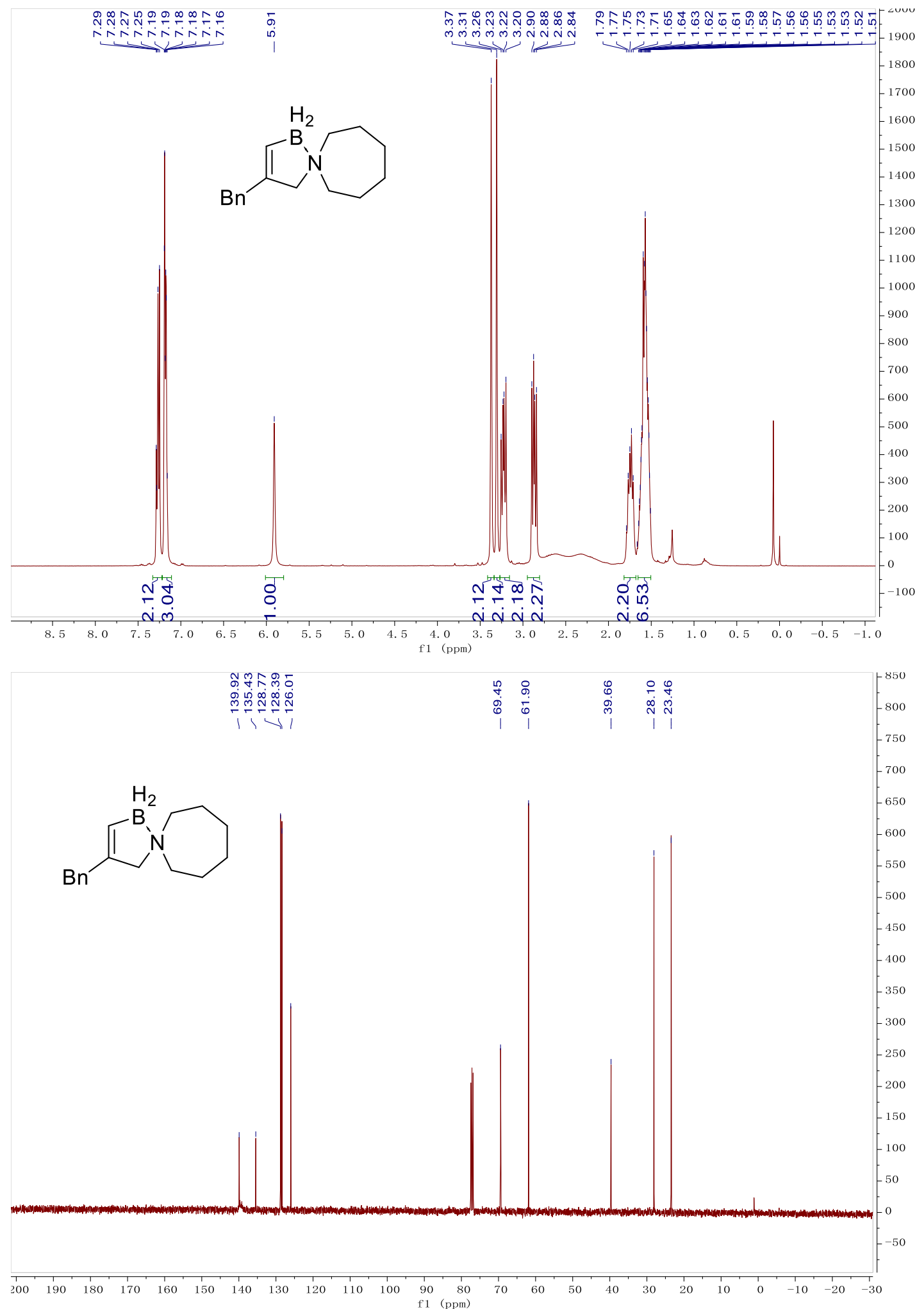


3-benzyl-8-oxa-5 $\lambda^{4}$-aza-1-boraspiro[4.5]dec-2-ene (5h)

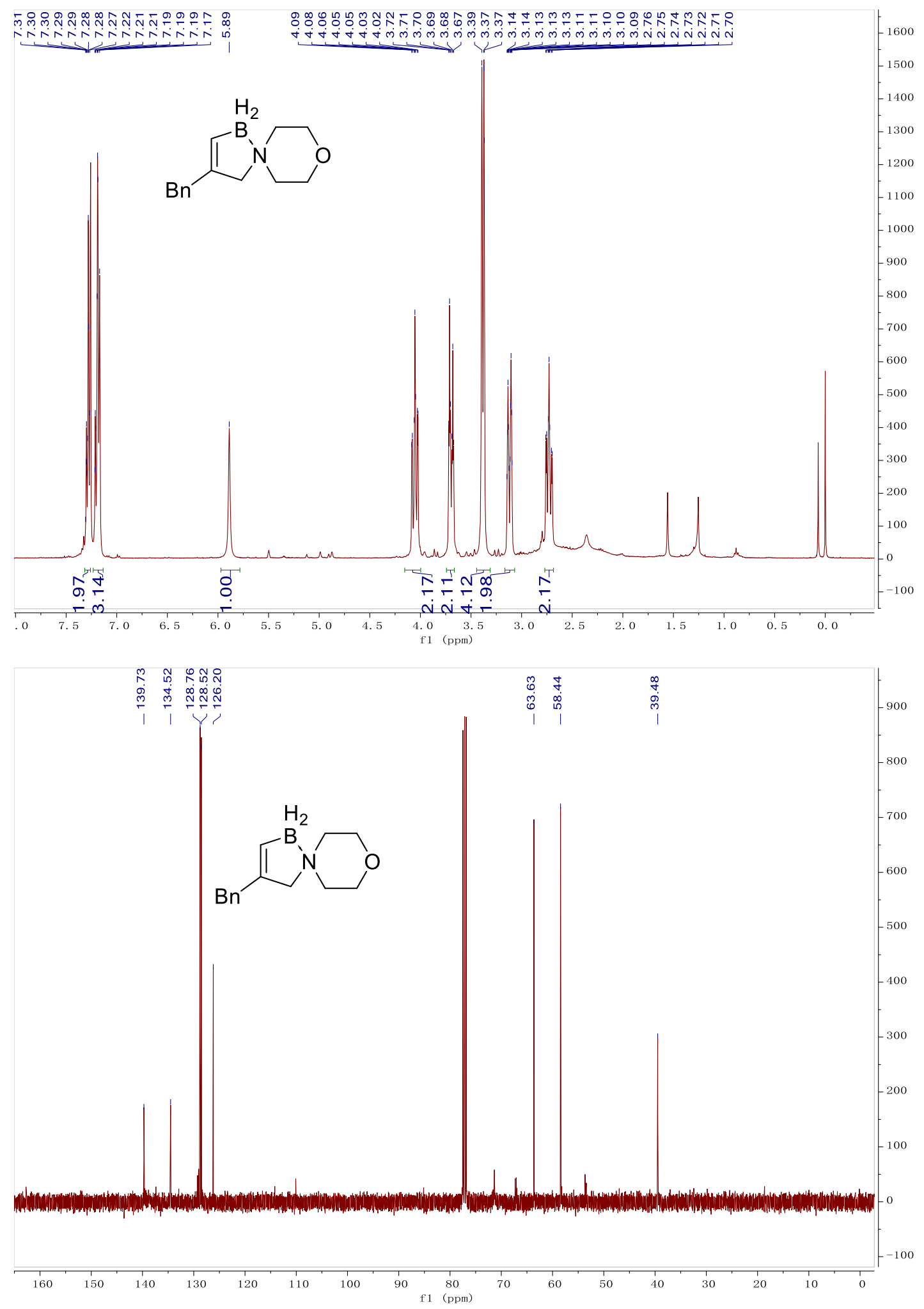




\section{1,4-dibenzyl-2,5-dihydro-1H-1,2-azaborole (5i)}

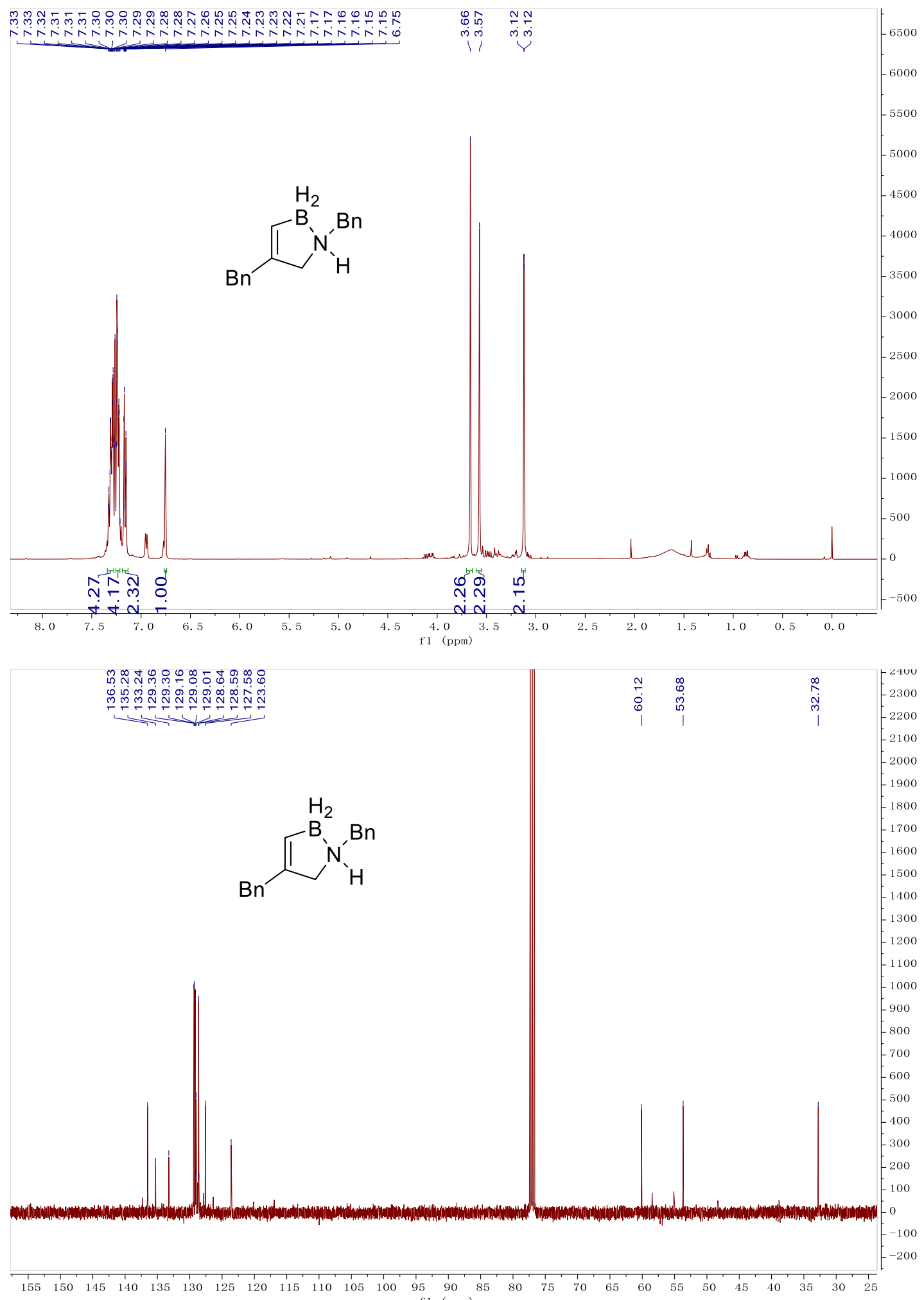


(S)-4-benzyl-1-(1-phenylethyl)-2,5-dihydro- $1 H-1 \lambda^{4}, 2$-azaborole (5j)

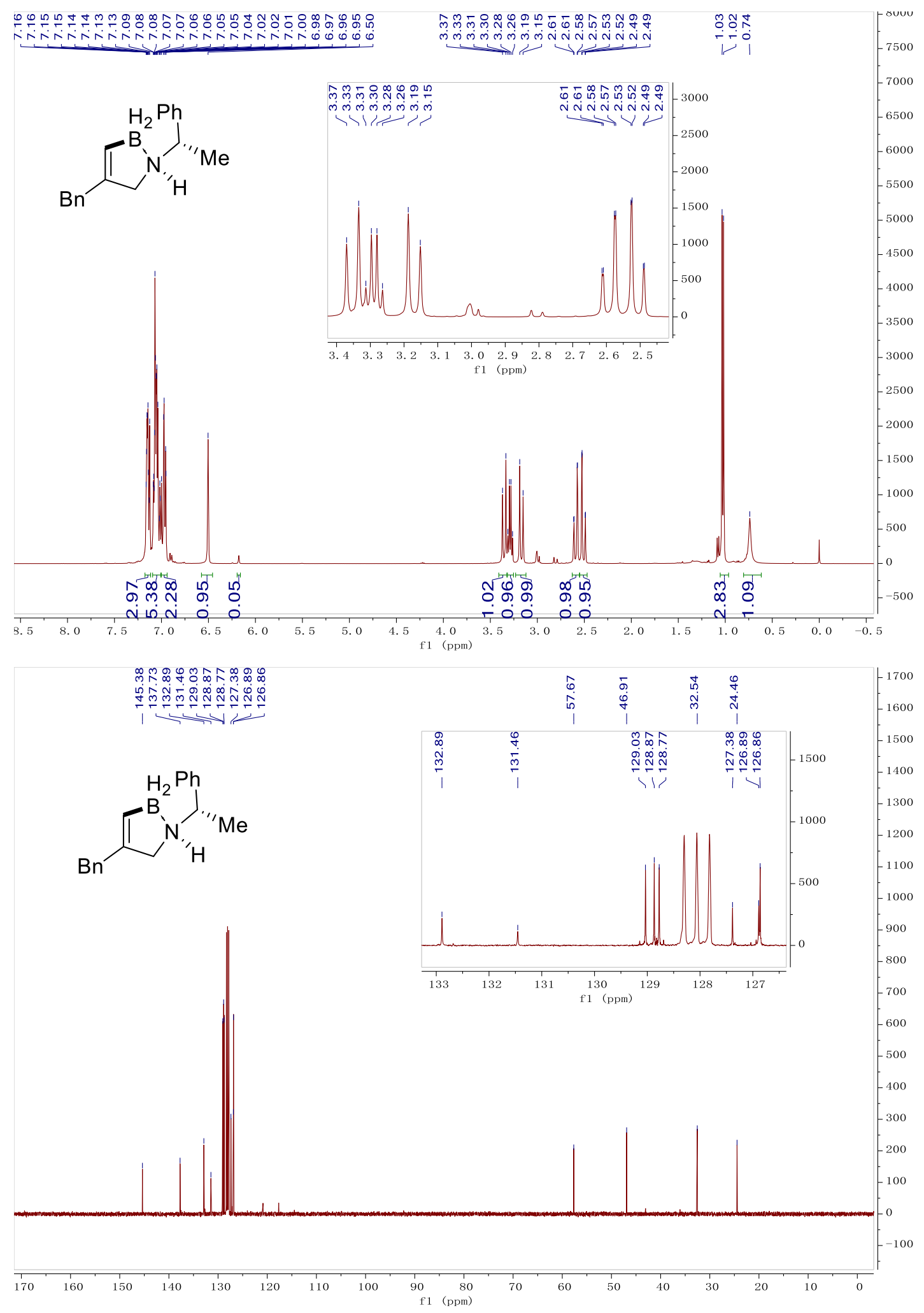



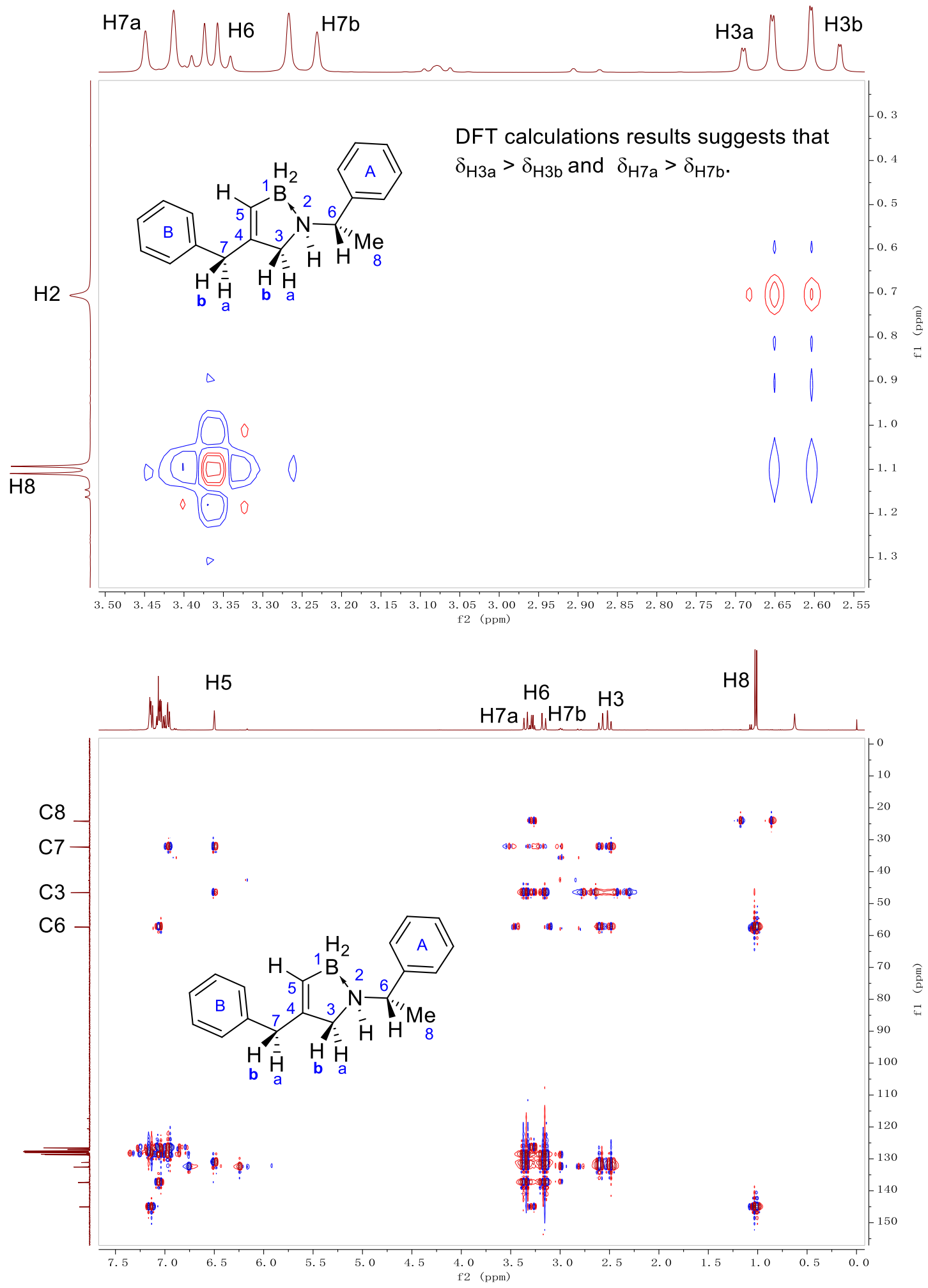
(7S,8R)-7-((benzo[d][1,3]dioxol-5-yloxy)methyl)-3-benzyl-8-(4-fluorophenyl)-5 $\lambda^{4}$ aza-1-boraspiro[4.5]dec-2-ene (5k)

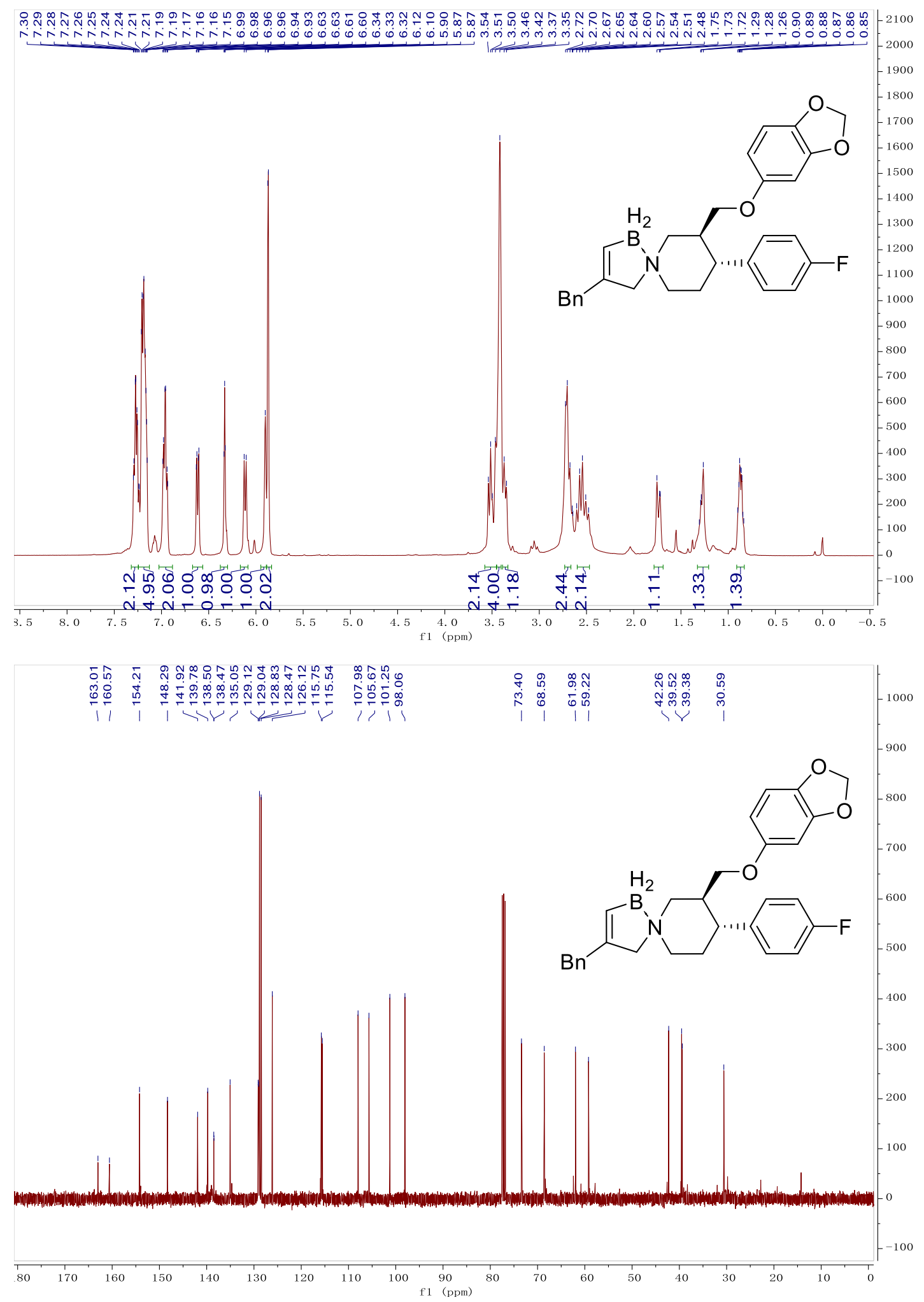


4-benzyl-1-(3-(10,11-dihydro-5H-dibenzo[a,d][7]annulen-5-ylidene)propyl)-1methyl-2,5-dihydro-1H-1 $\lambda^{4}, 2$-azaborole (5l)

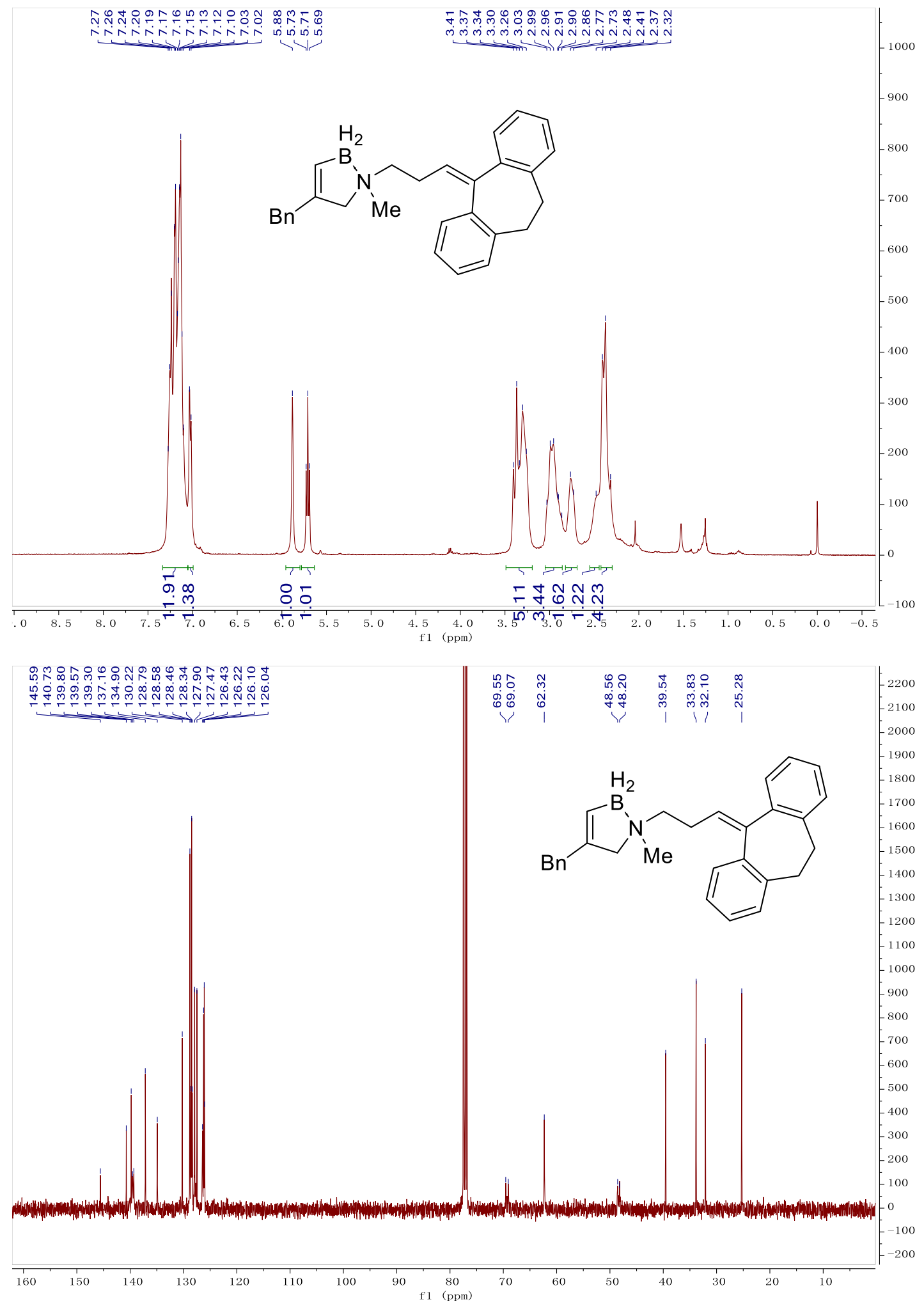


4-benzyl-1-methyl-1-(3-phenyl-3-(4-(trifluoromethyl)phenoxy)propyl)-2,5-

dihydro- $1 H-1 \lambda^{4}, 2$-azaborole $(5 \mathrm{~m})$

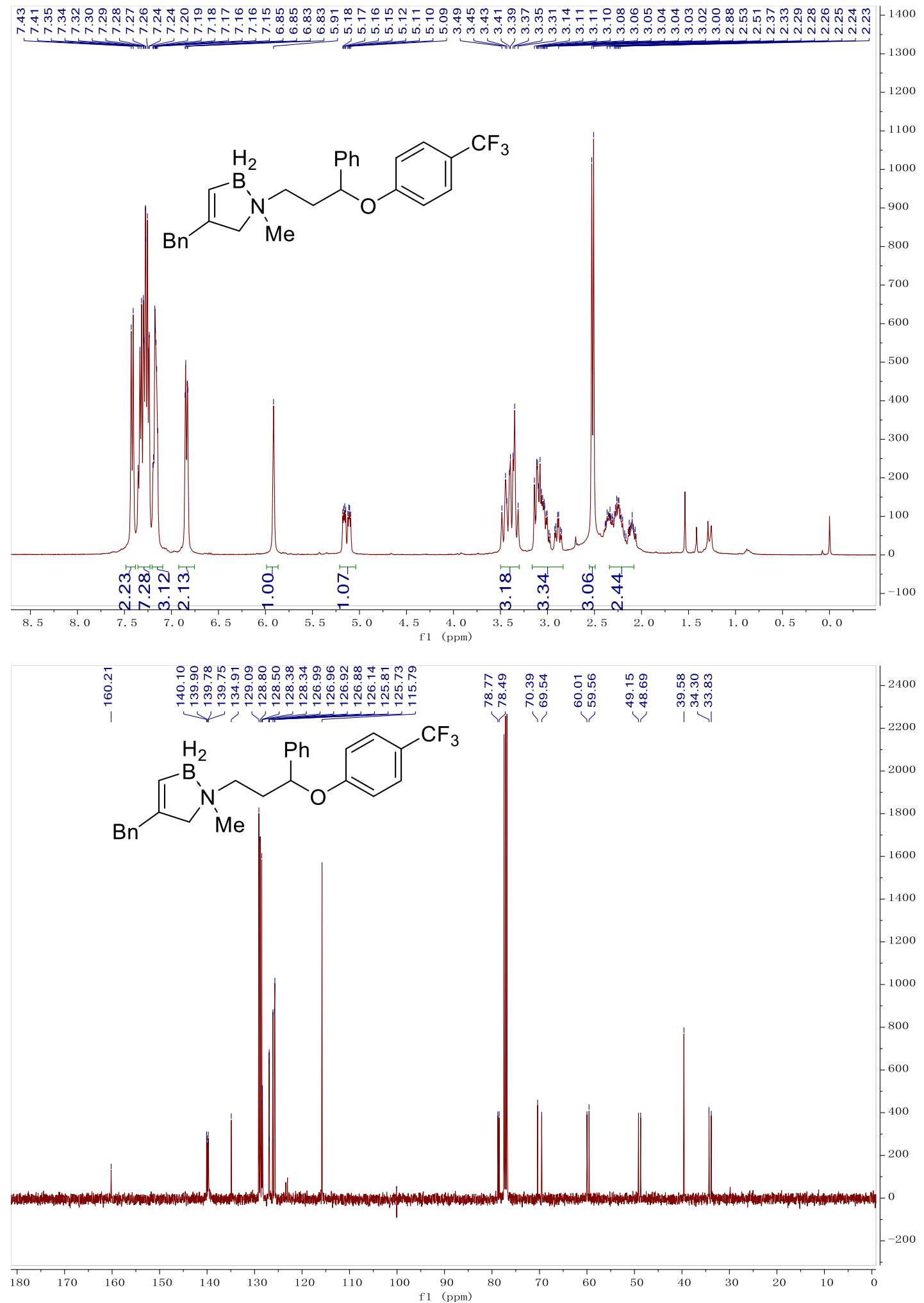




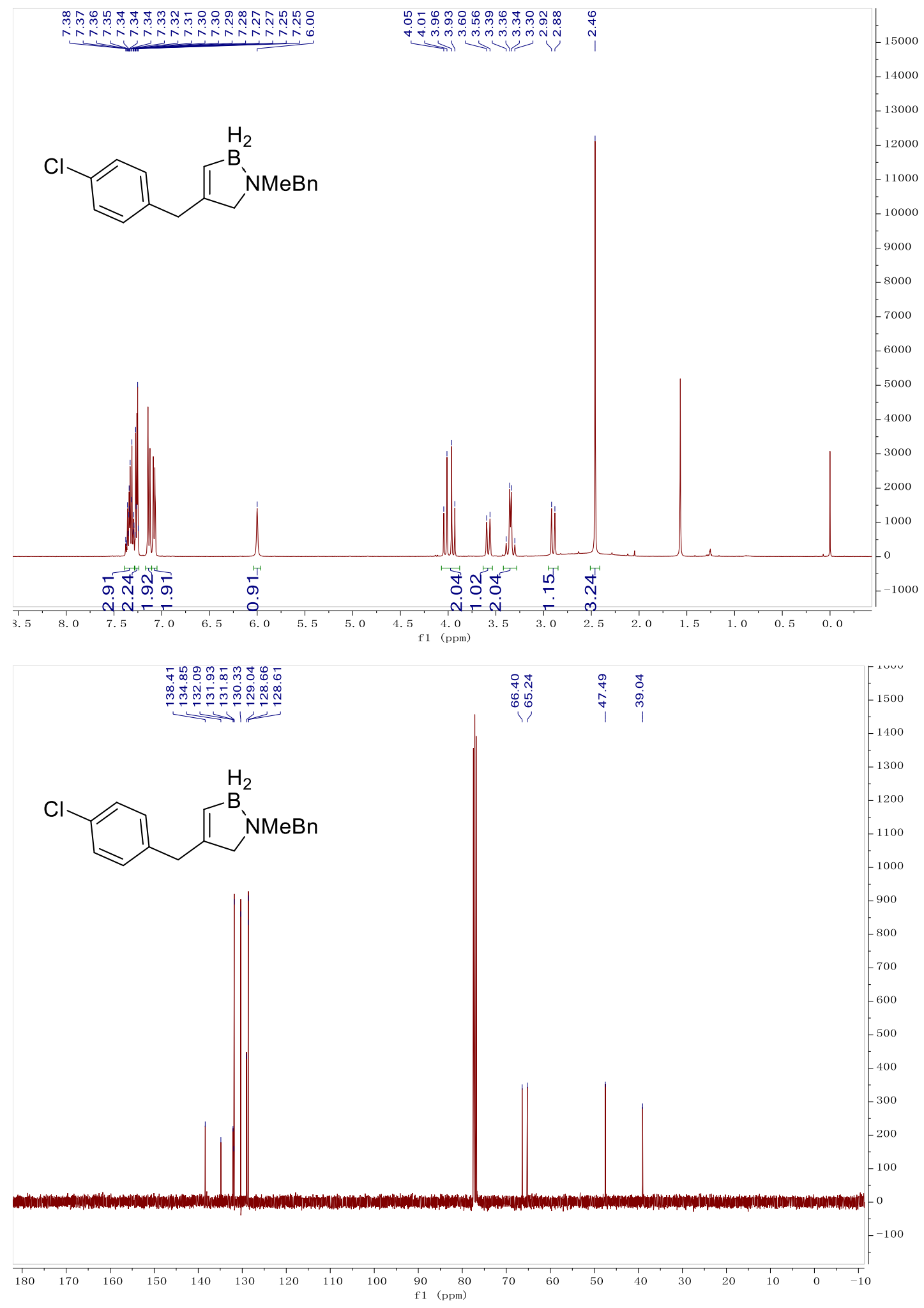



(5o)

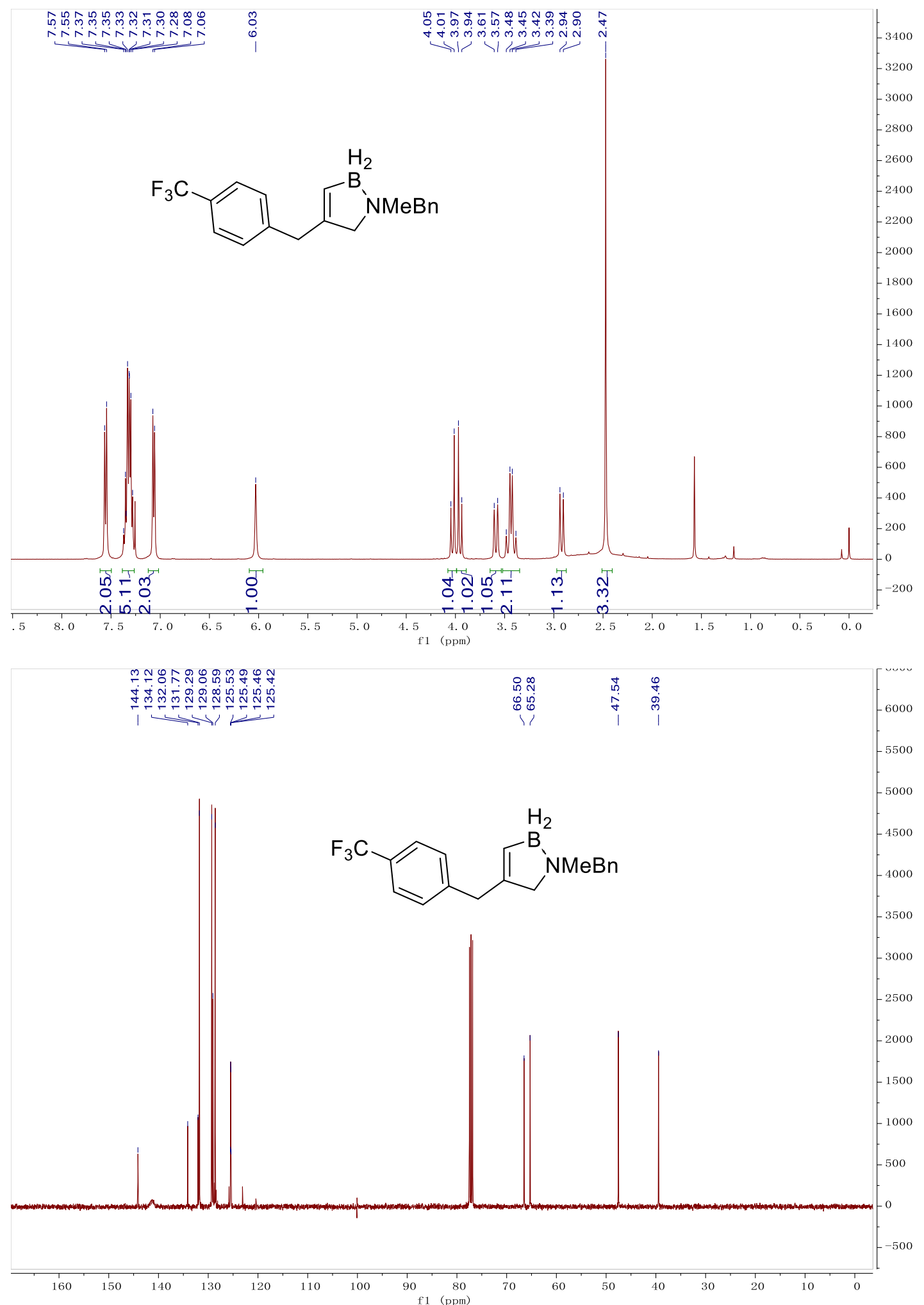


1-benzyl-4-(4-methoxybenzyl)-1-methyl-2,5-dihydro- $1 H$ - $1 \lambda^{4}, 2$-azaborole (5p)

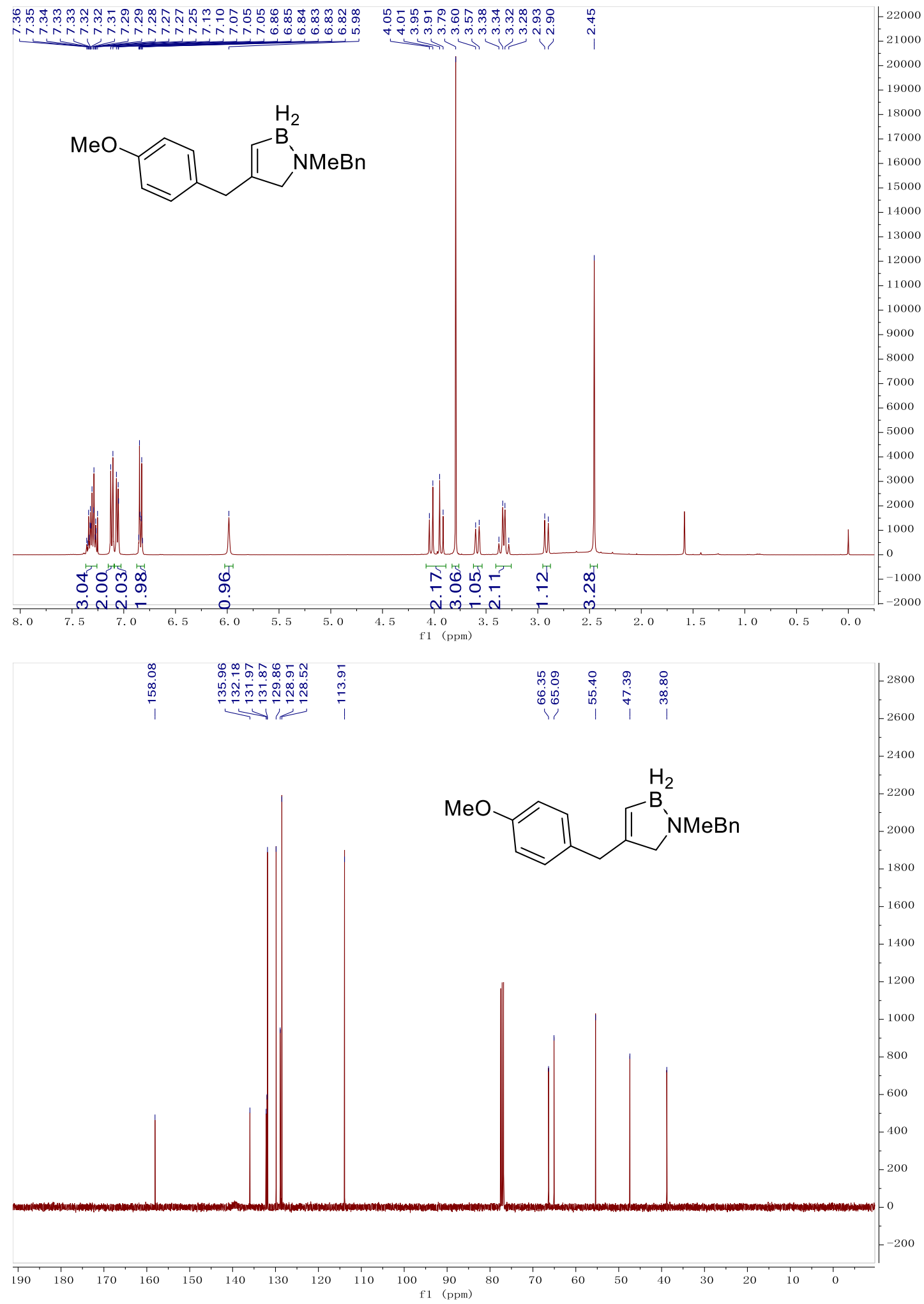


1-benzyl-4-(4-fluorobenzyl)-1-methyl-2,5-dihydro-1 $H$-1 $\lambda^{4}, 2$-azaborole (5q)

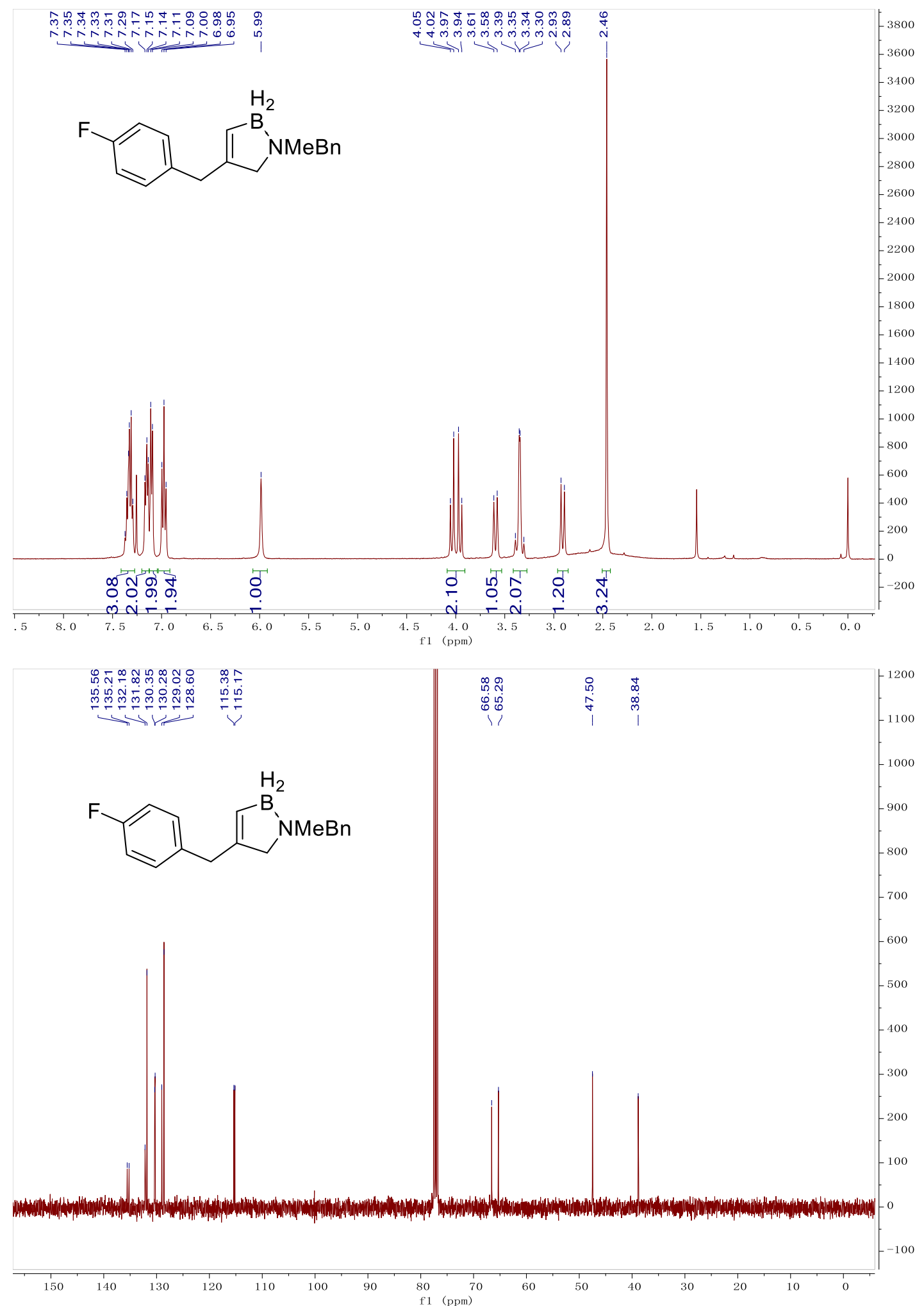


1-benzyl-1,4-dimethyl-2,5-dihydro- $1 H$ - $1 \lambda^{4}, 2$-azaborole (5r)

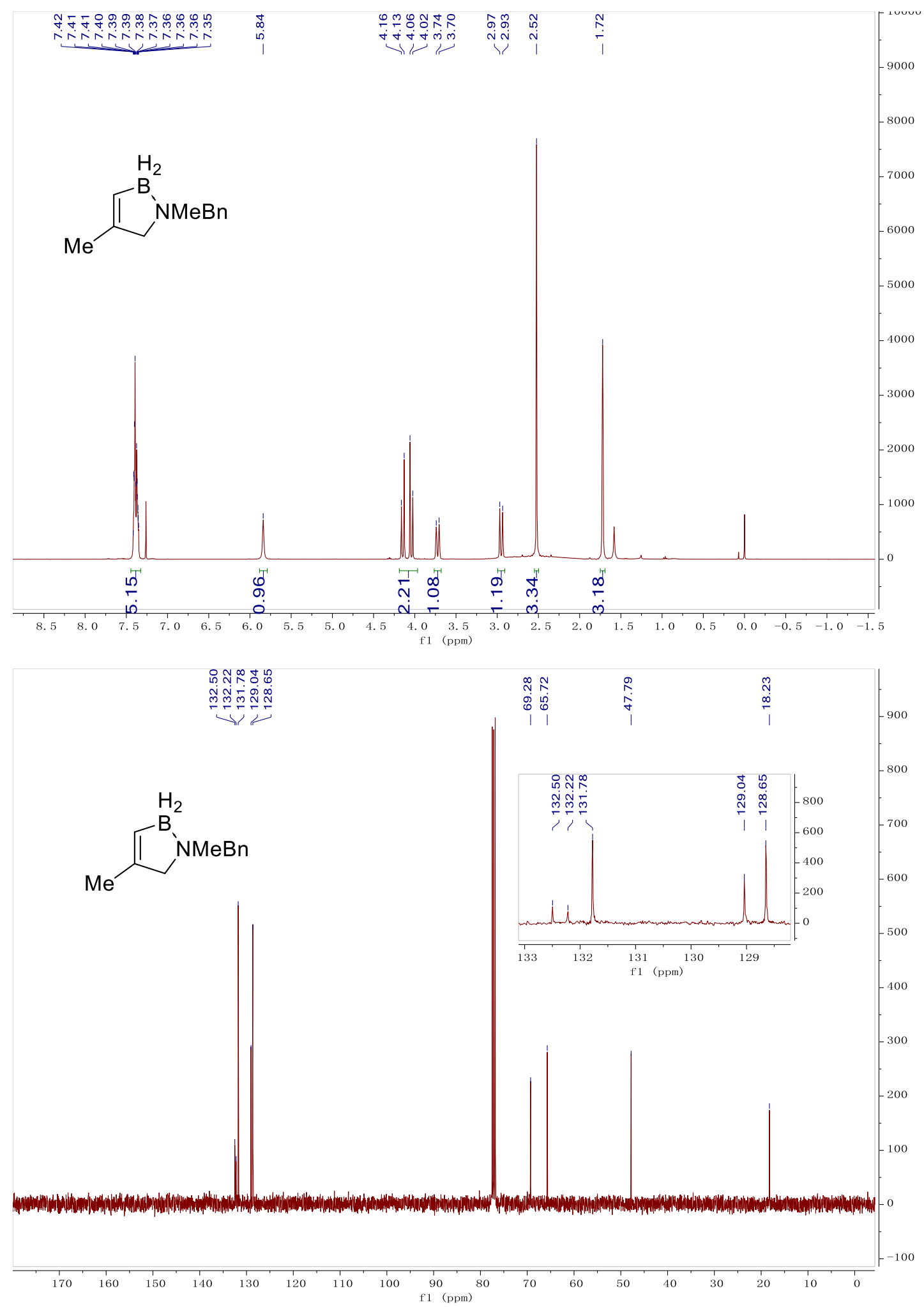


1-benzyl-4-butyl-1-methyl-2,5-dihydro- $1 H-1 \lambda^{4}, 2$-azaborole (5s)

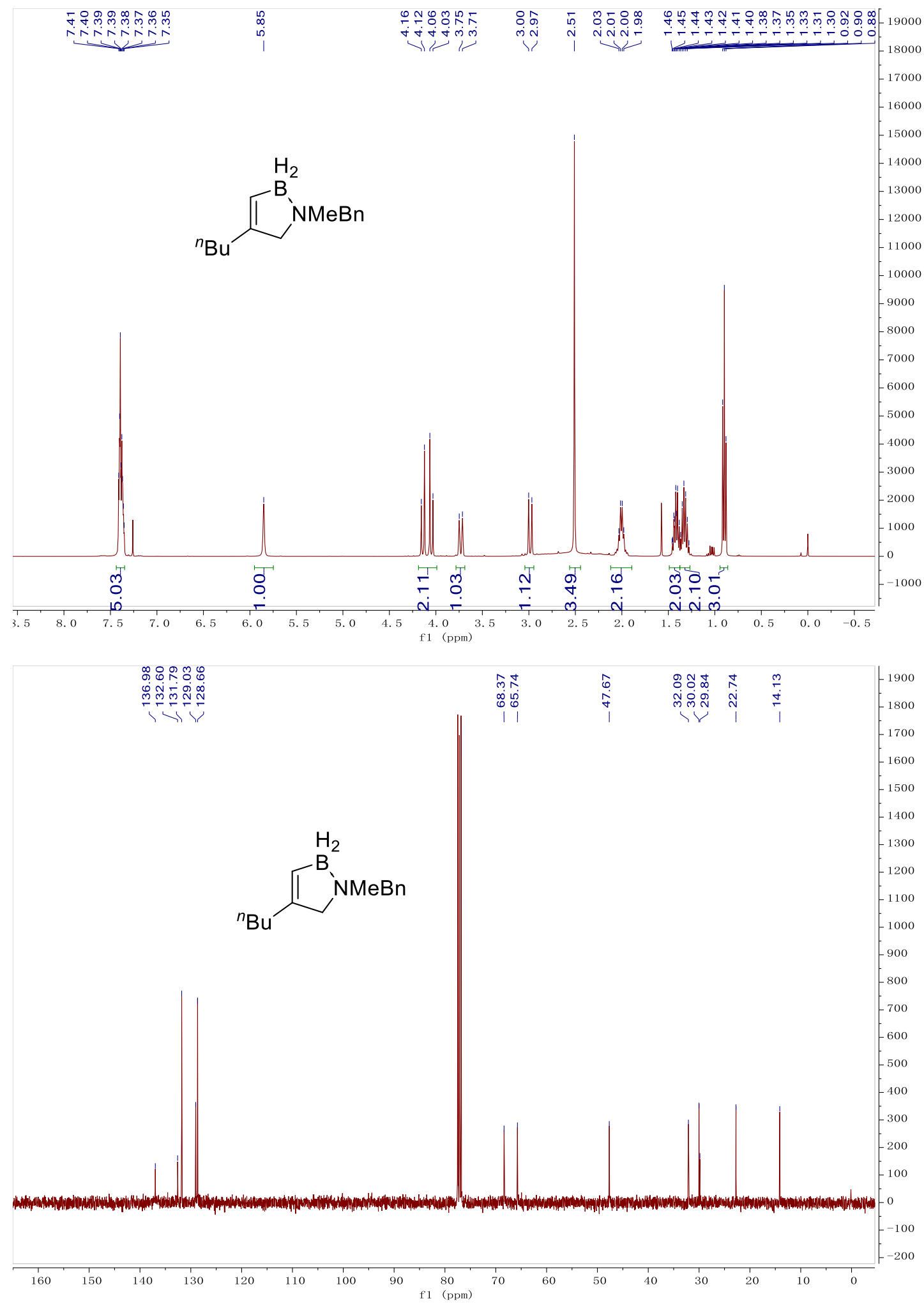


1-benzyl-4-isopropyl-1-methyl-2,5-dihydro- $1 H$-1 $1 \lambda^{4}, 2$-azaborole (5t)

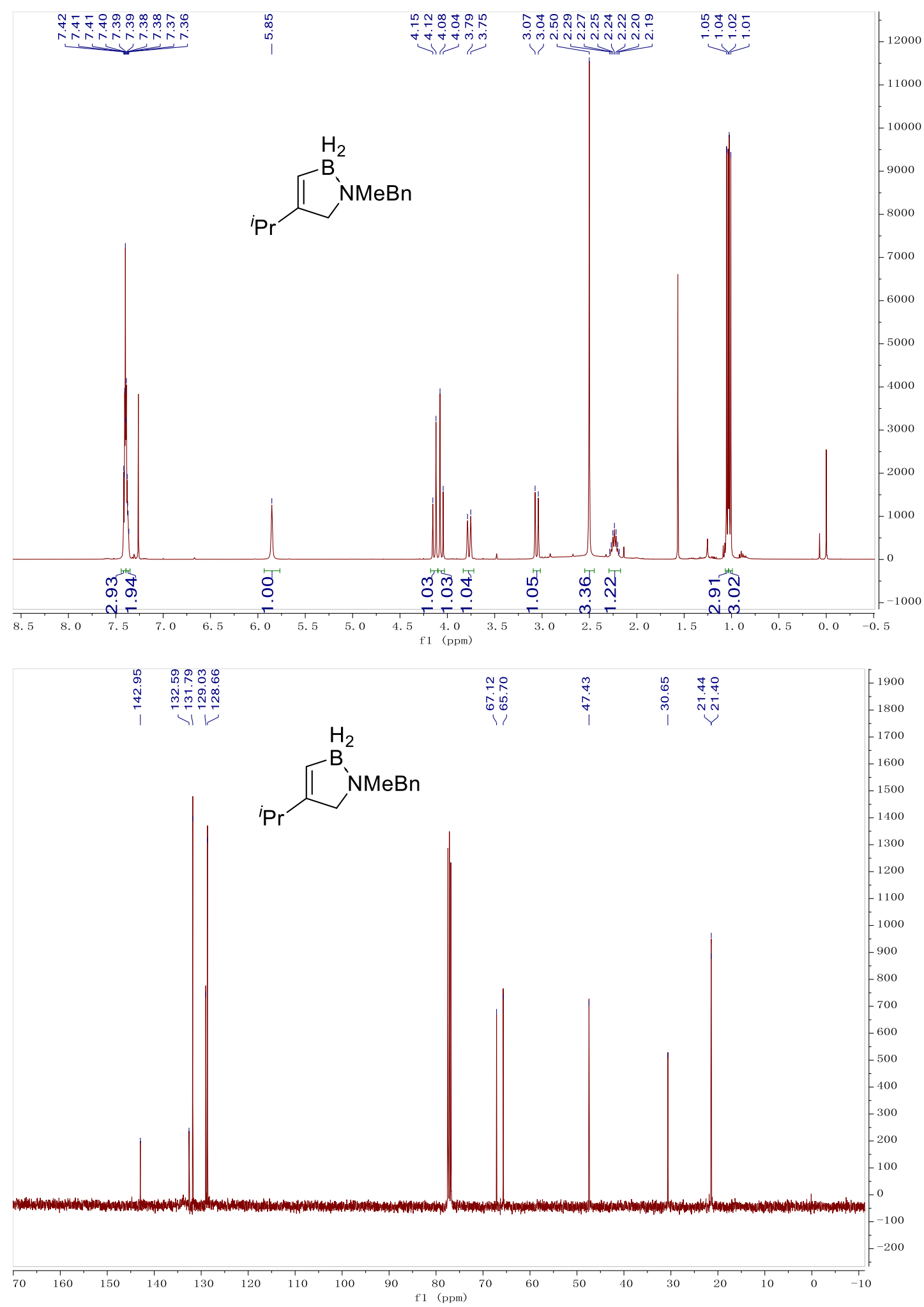




\section{1-benzyl-4-cyclohexyl-1-methyl-2,5-dihydro- $1 H$ - $1 \lambda^{4}, 2$-azaborole (5u)}

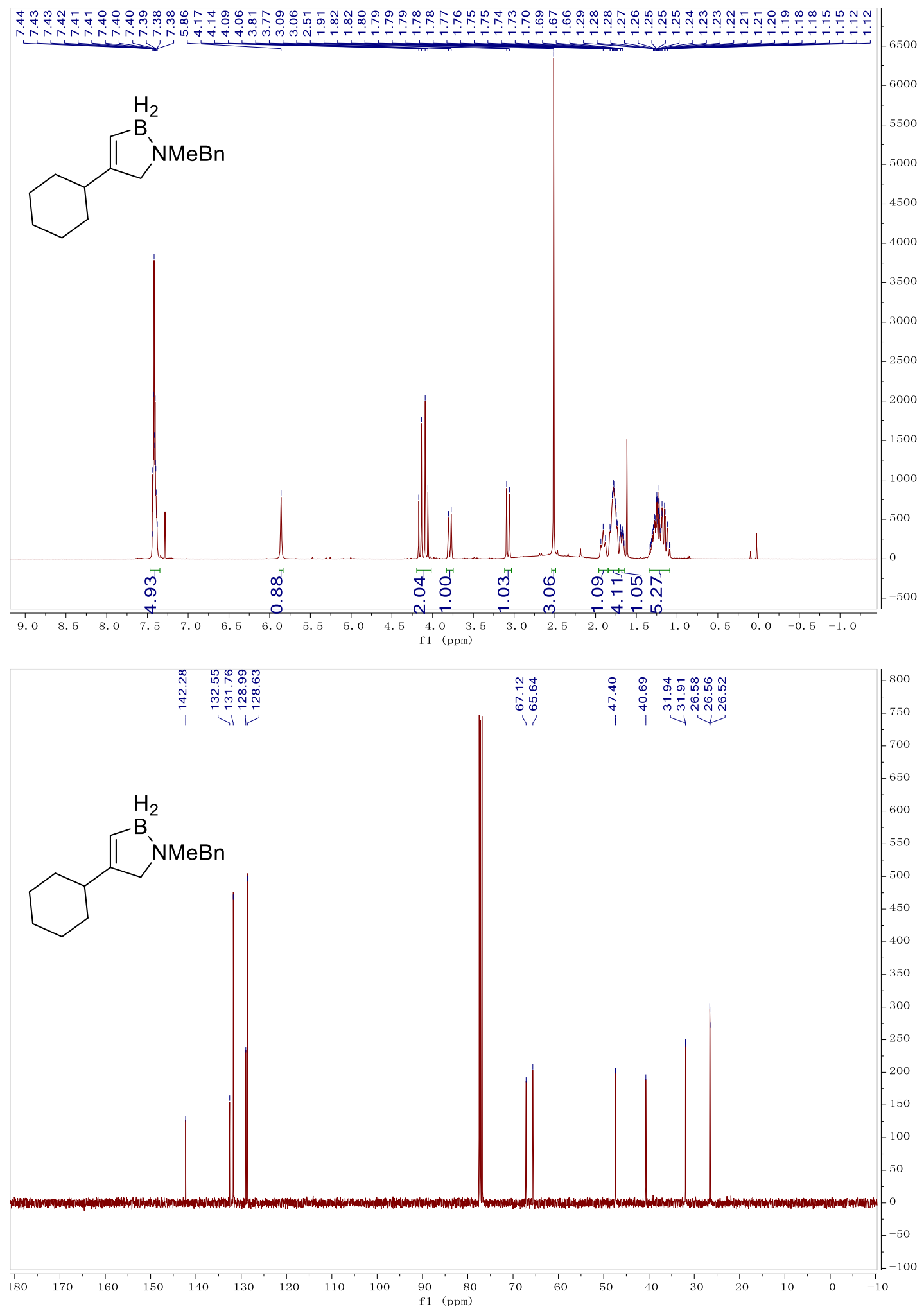


1,1-dimethyl-4-phenyl-2,5-dihydro- $1 H-1 \lambda^{4}, 2$-azaborole (5v)

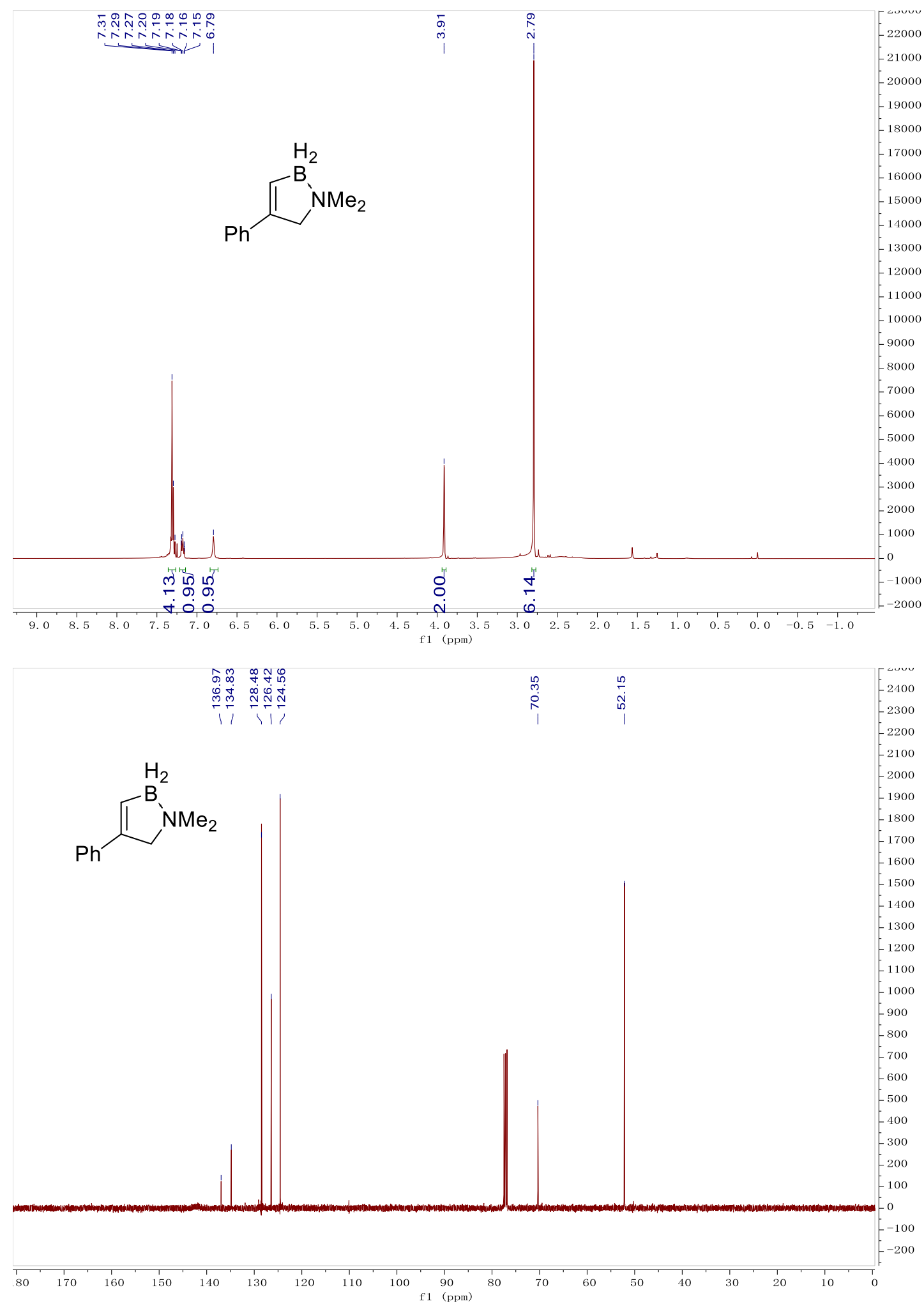


4-(4-methoxyphenyl)-1,1-dimethyl-2,5-dihydro-1 $H$-1 $\lambda^{4}, 2$-azaborole (5w)

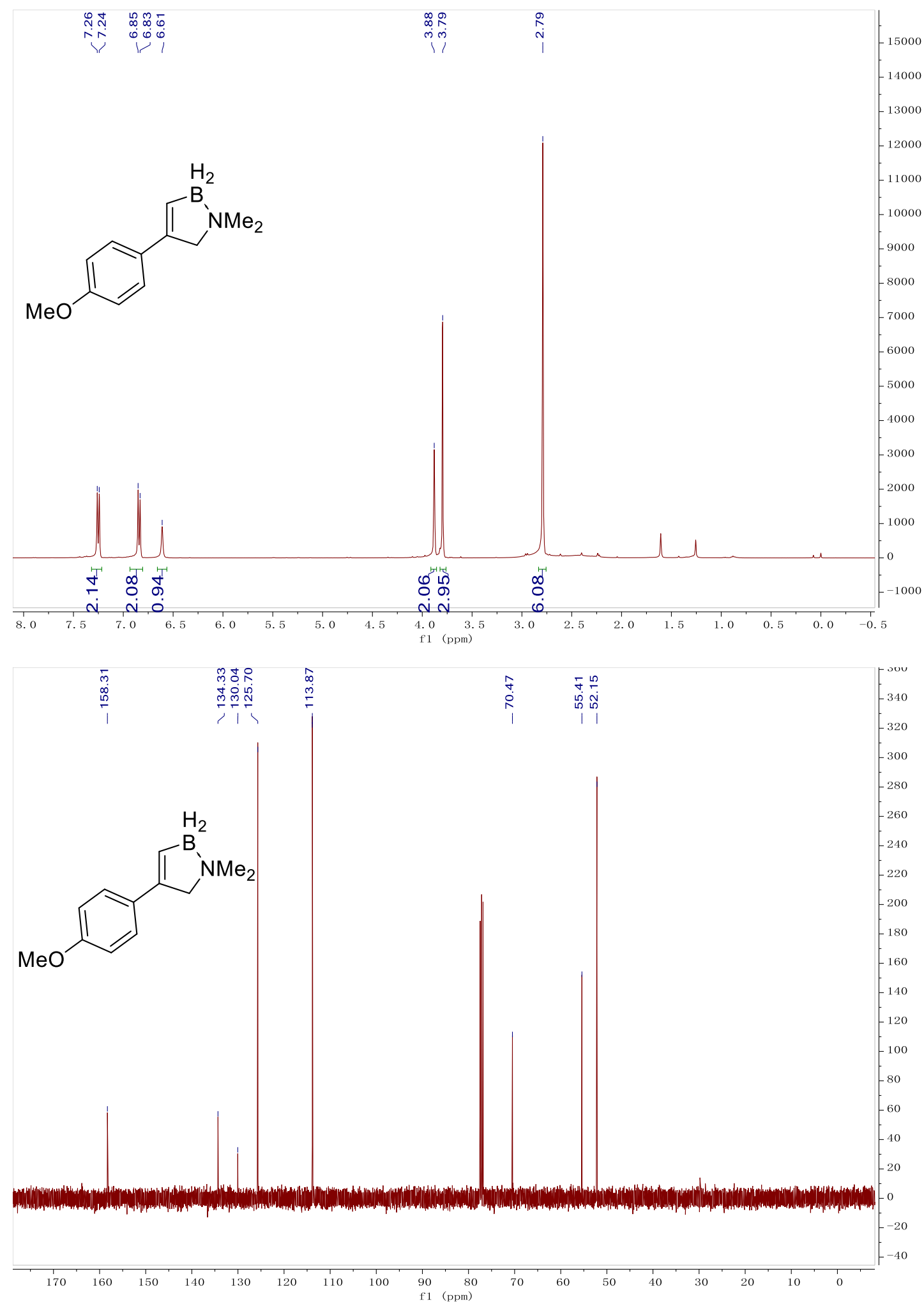


4-(4-chlorophenyl)-1,1-dimethyl-2,5-dihydro-1 $H-1 \lambda^{4}, 2$-azaborole (5x)

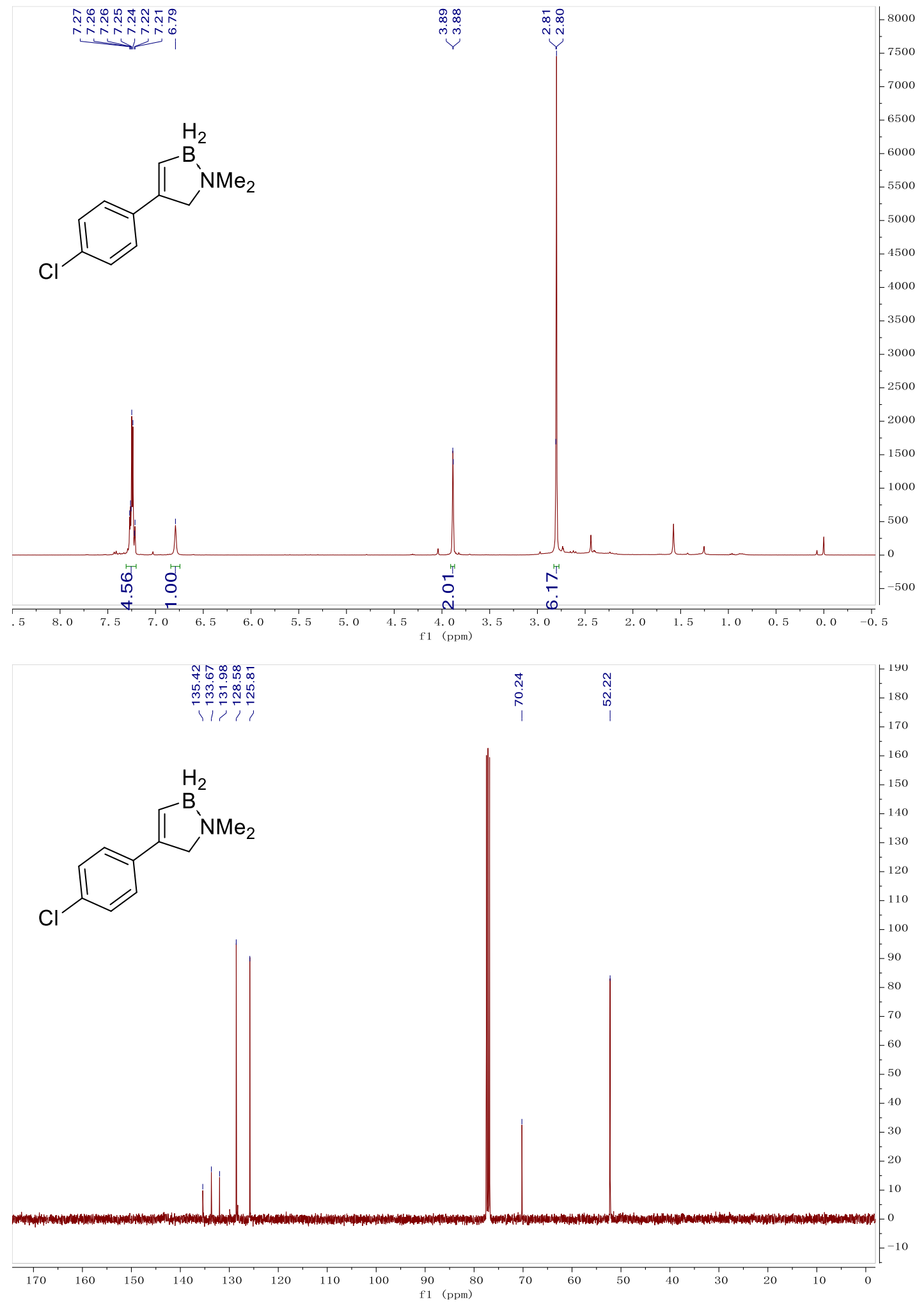




\section{4-(4-trifluoromethy)-1,1-dimethyl-2,5-dihydro- $1 H$-1 $\lambda^{4}, 2$-azaborole (5y)}

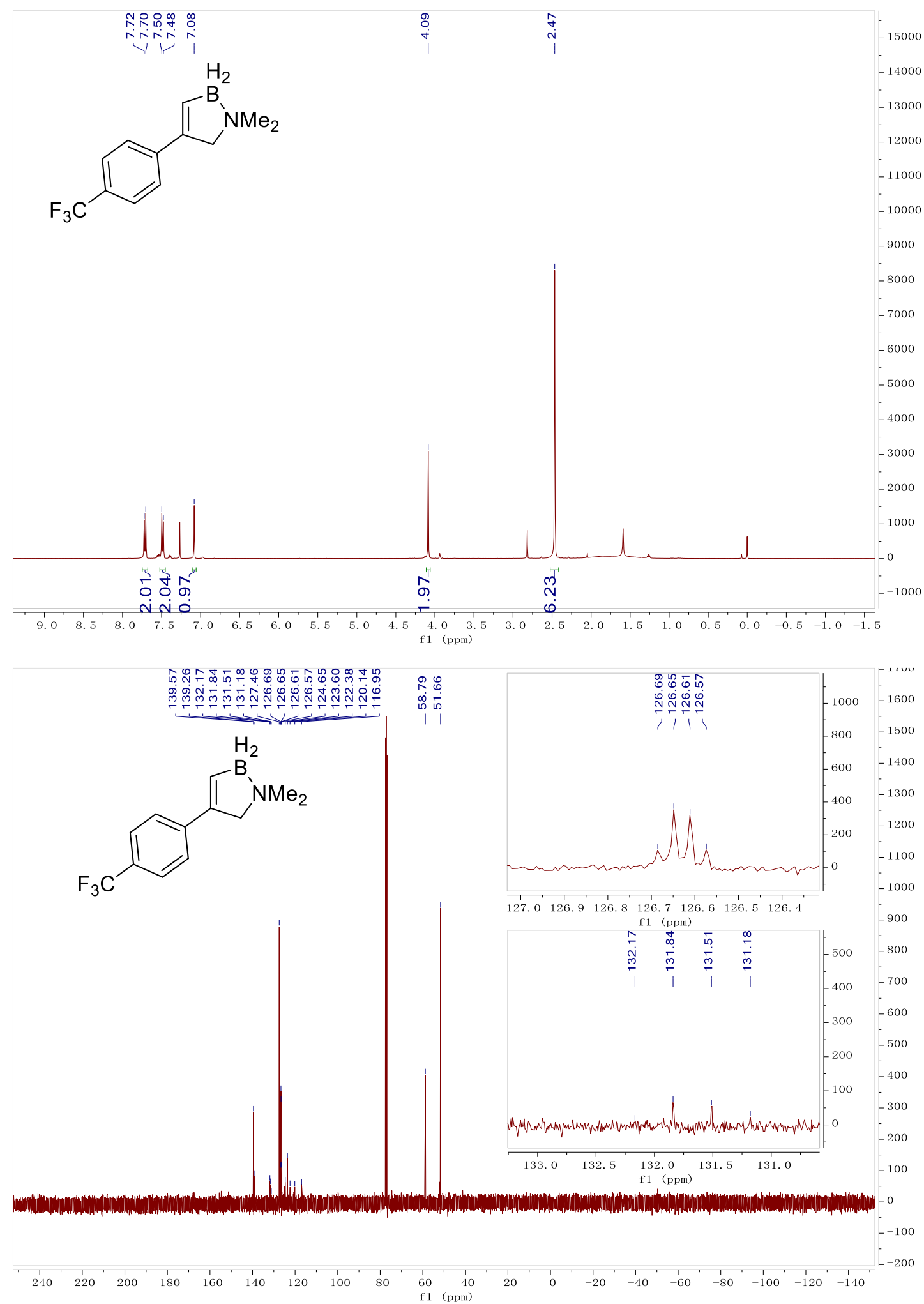


(4-benzylidenecyclohexyl)benzene (6)

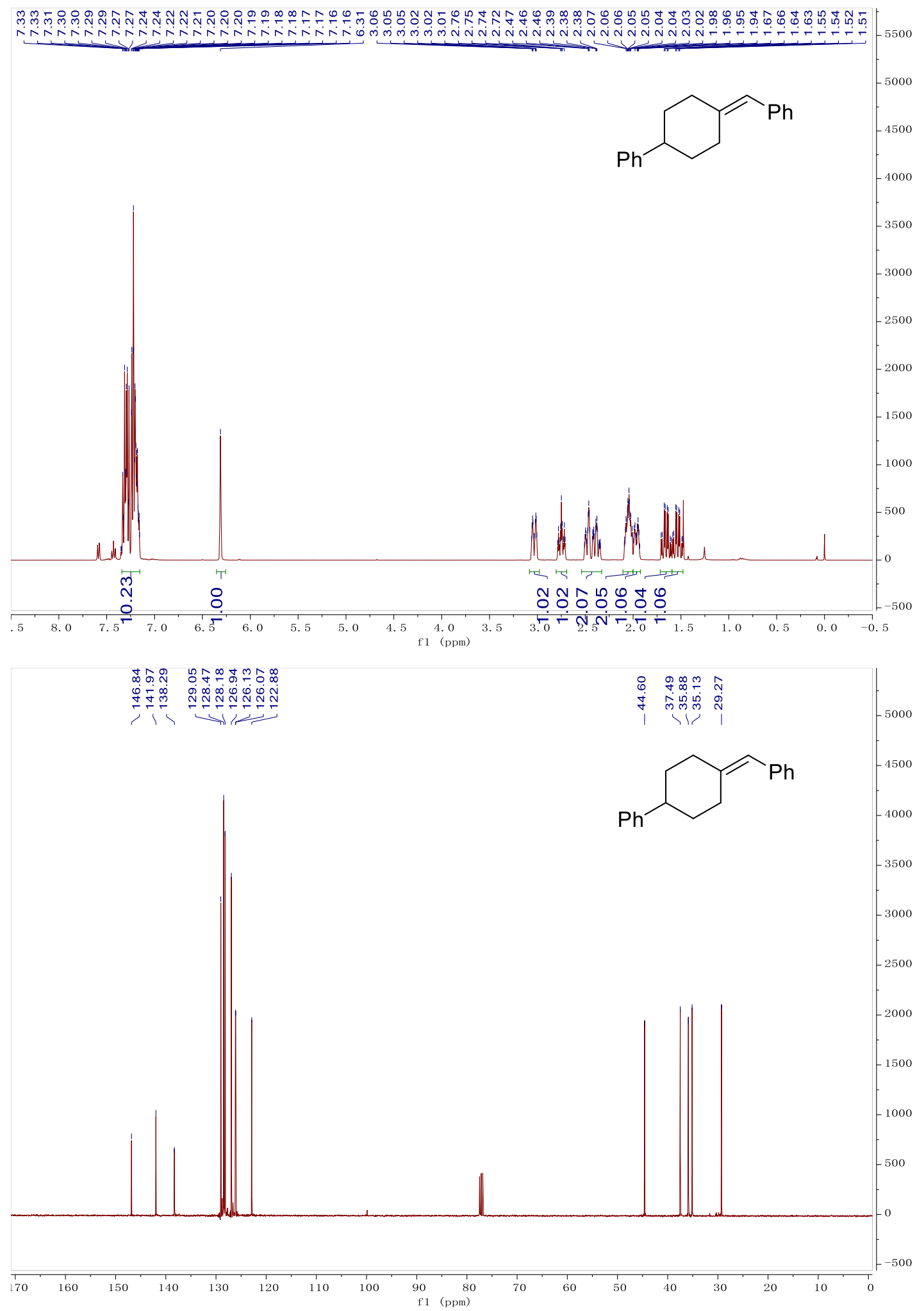




\section{(4-(3-methylbut-2-en-1-ylidene)cyclohexyl)benzene (7)}

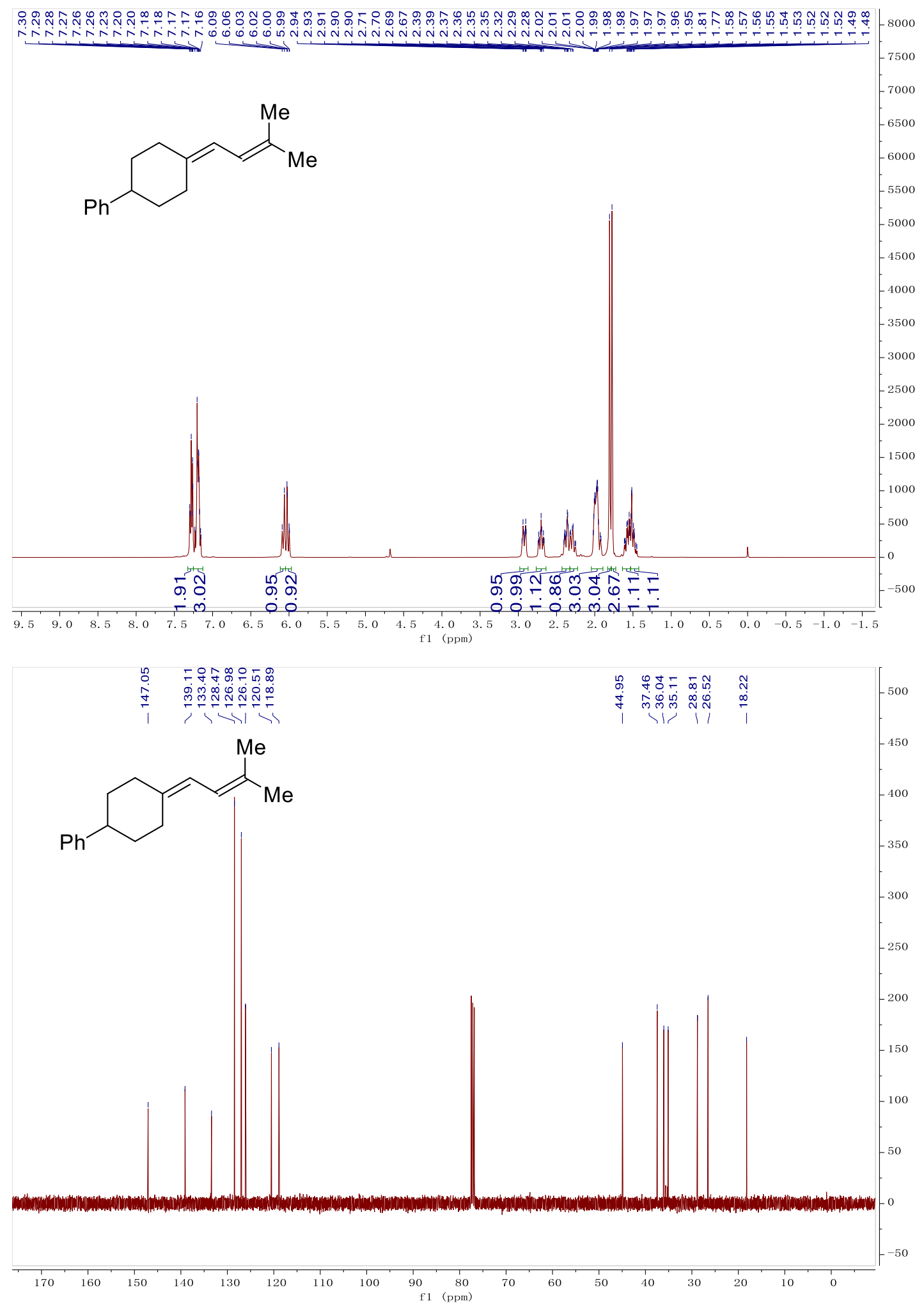


1-((4-phenylcyclohexylidene)methyl)-1H-imidazole (8)

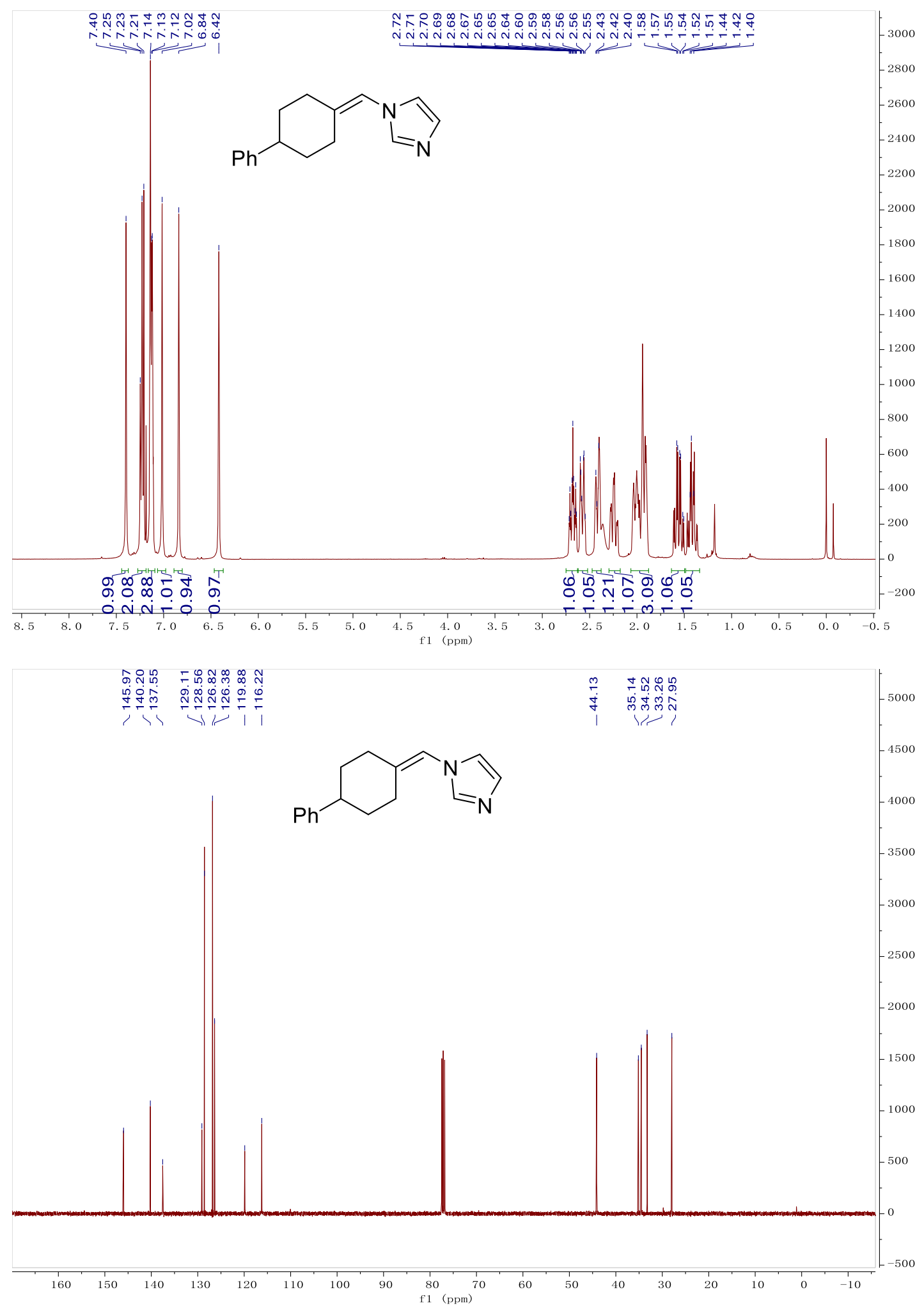


(4-(chloromethylene)cyclohexyl)benzene (9a)

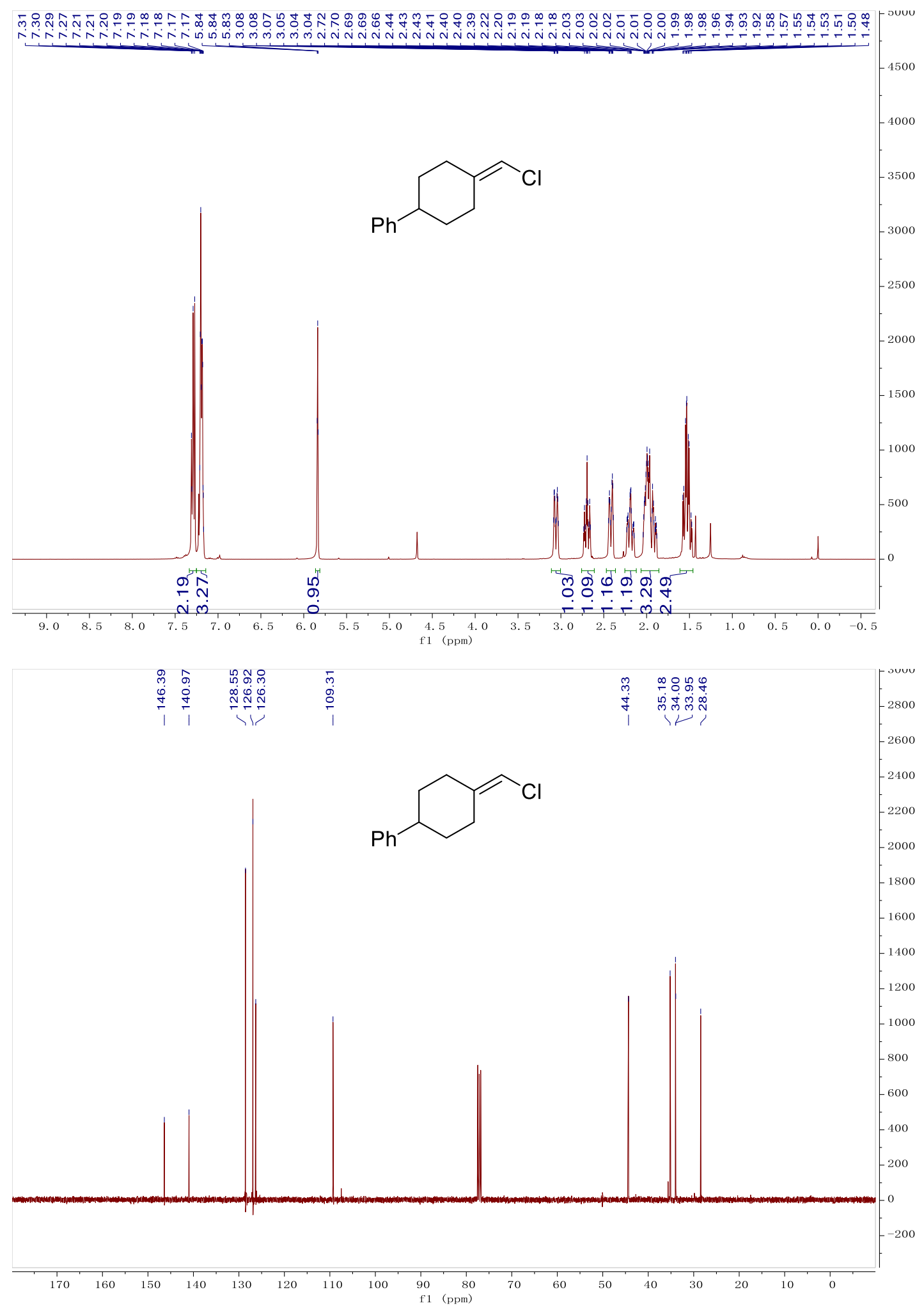


(4-(bromomethylene)cyclohexyl)benzene (9b)

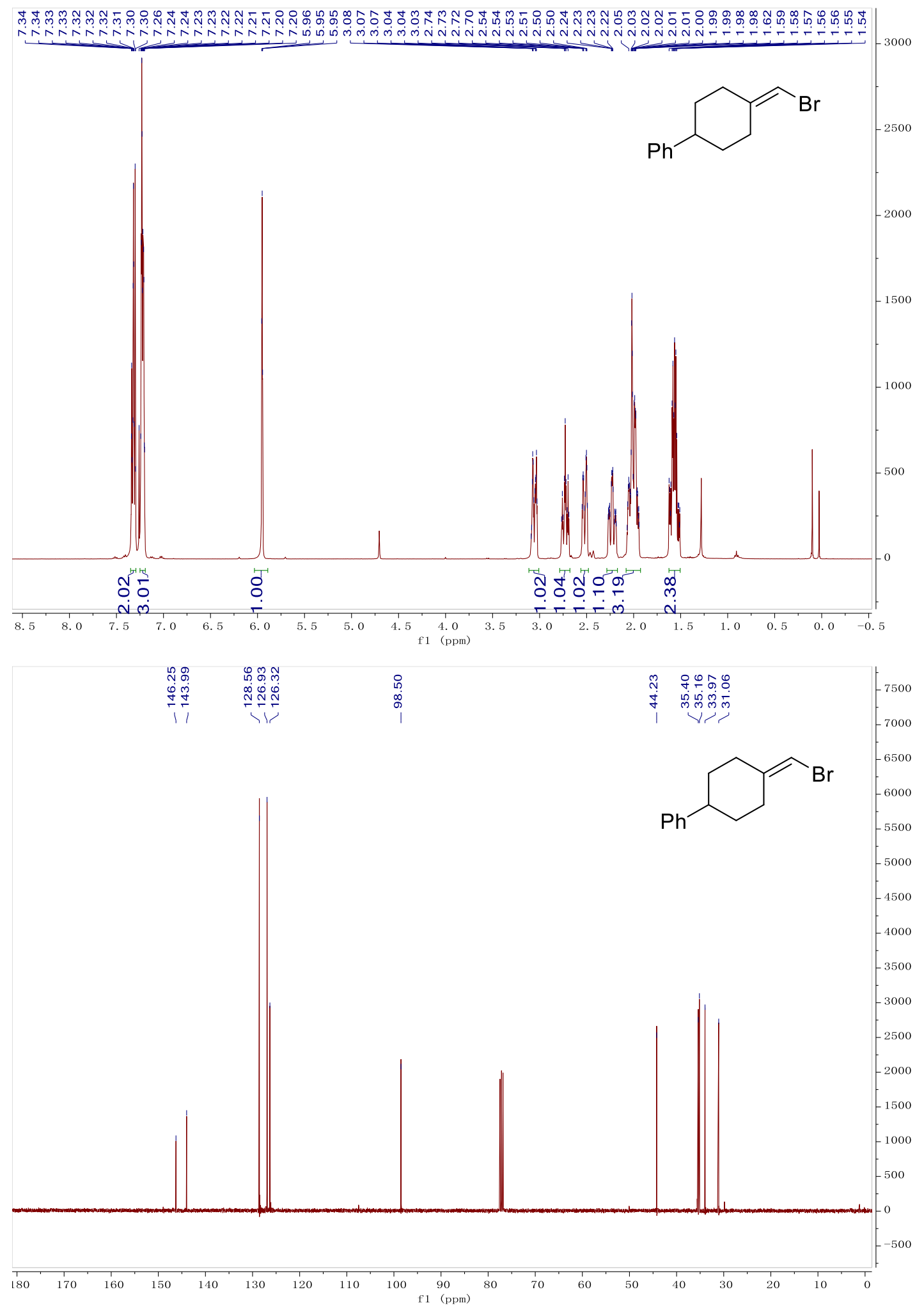


4,4,5,5-tetramethyl-2-((4-phenylcyclohexylidene)methyl)-1,3,2-dioxaborolane (10)

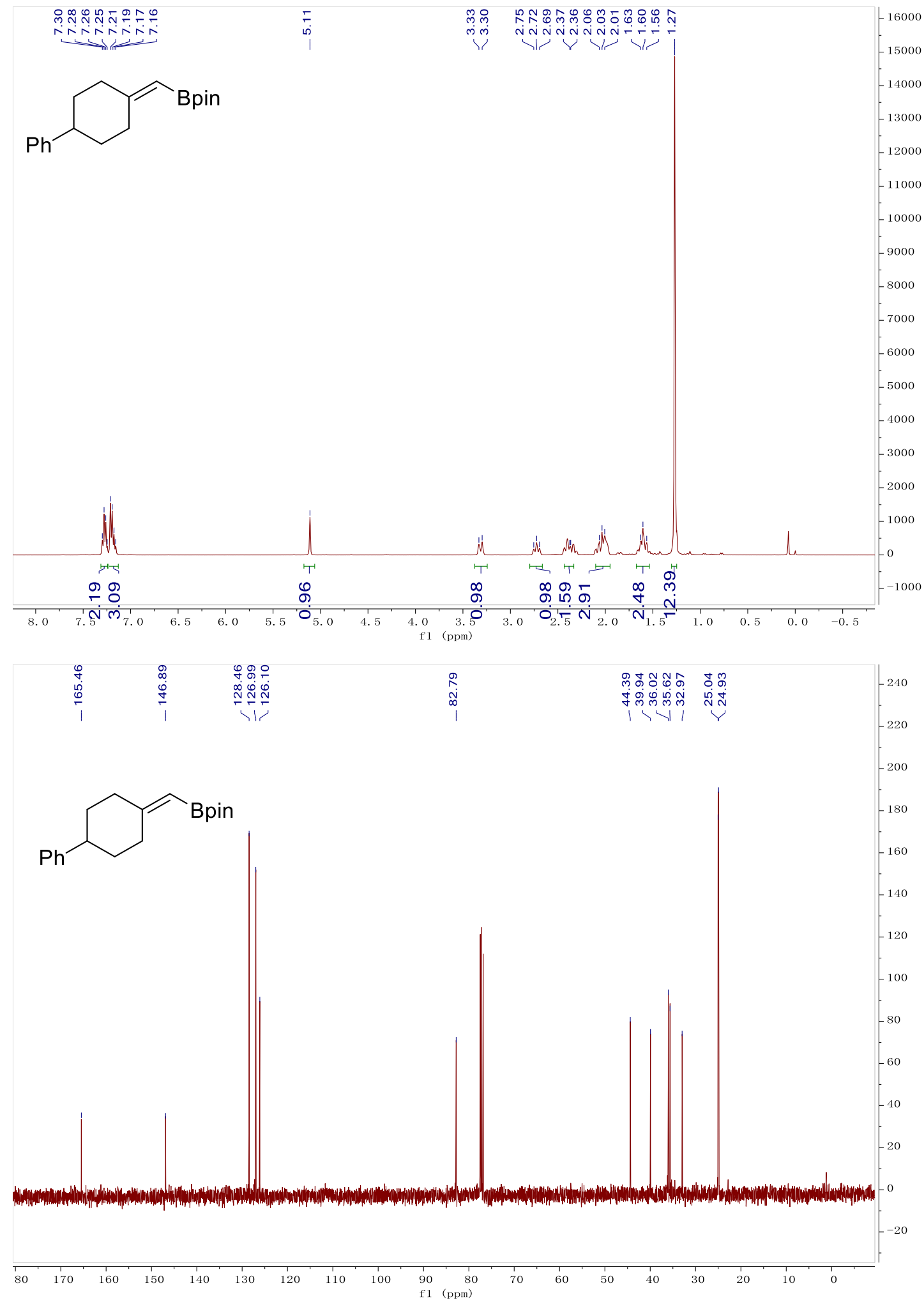


4-methyl-8-((4-phenylcyclohexylidene)methyl)dihydro- $4 \lambda^{4}, 8 \lambda^{4}-$

\section{[1,3,2] oxazaborolo[2,3-b][1,3,2] oxazaborole-2,6(3H,5H)-dione (11)}

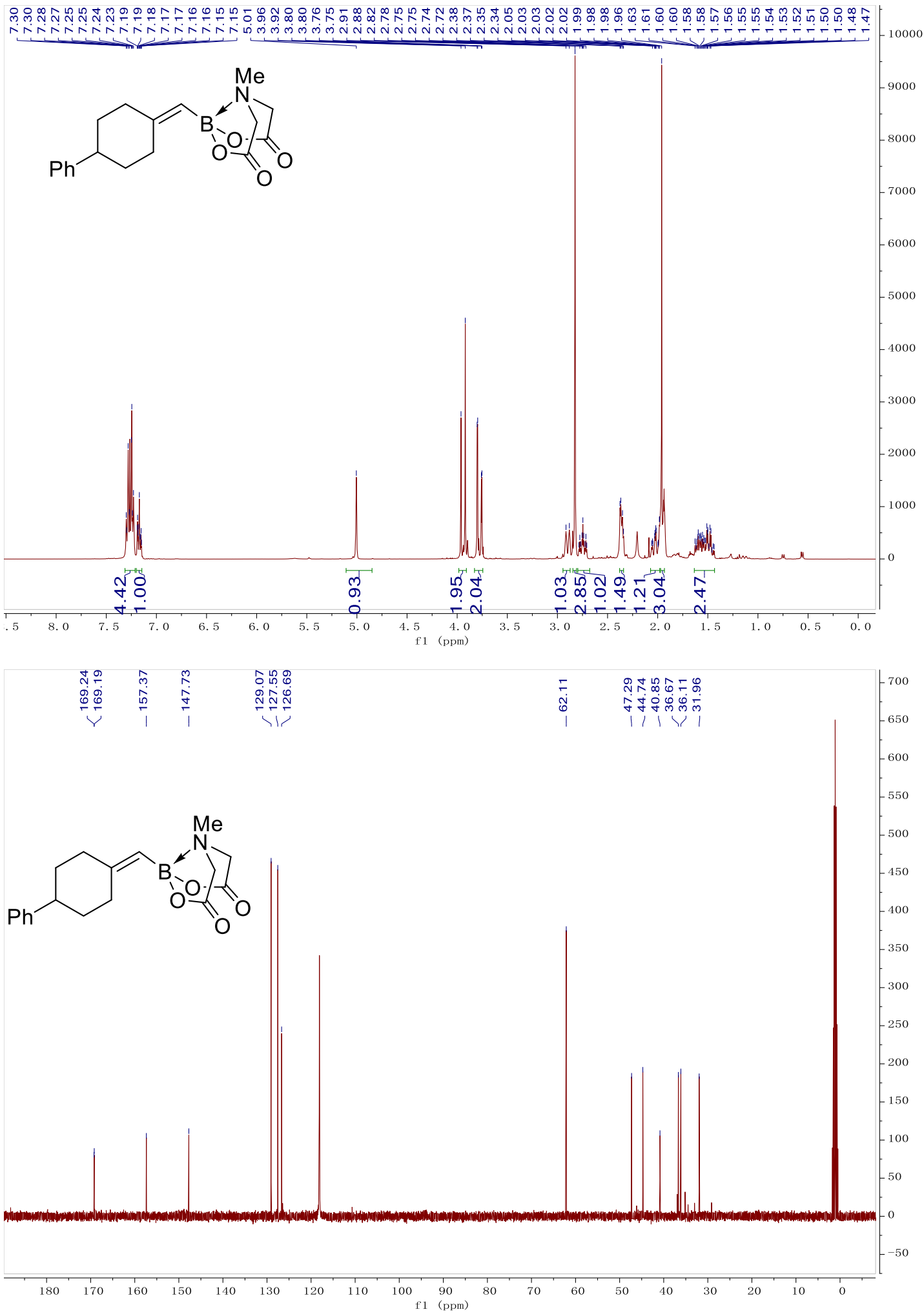




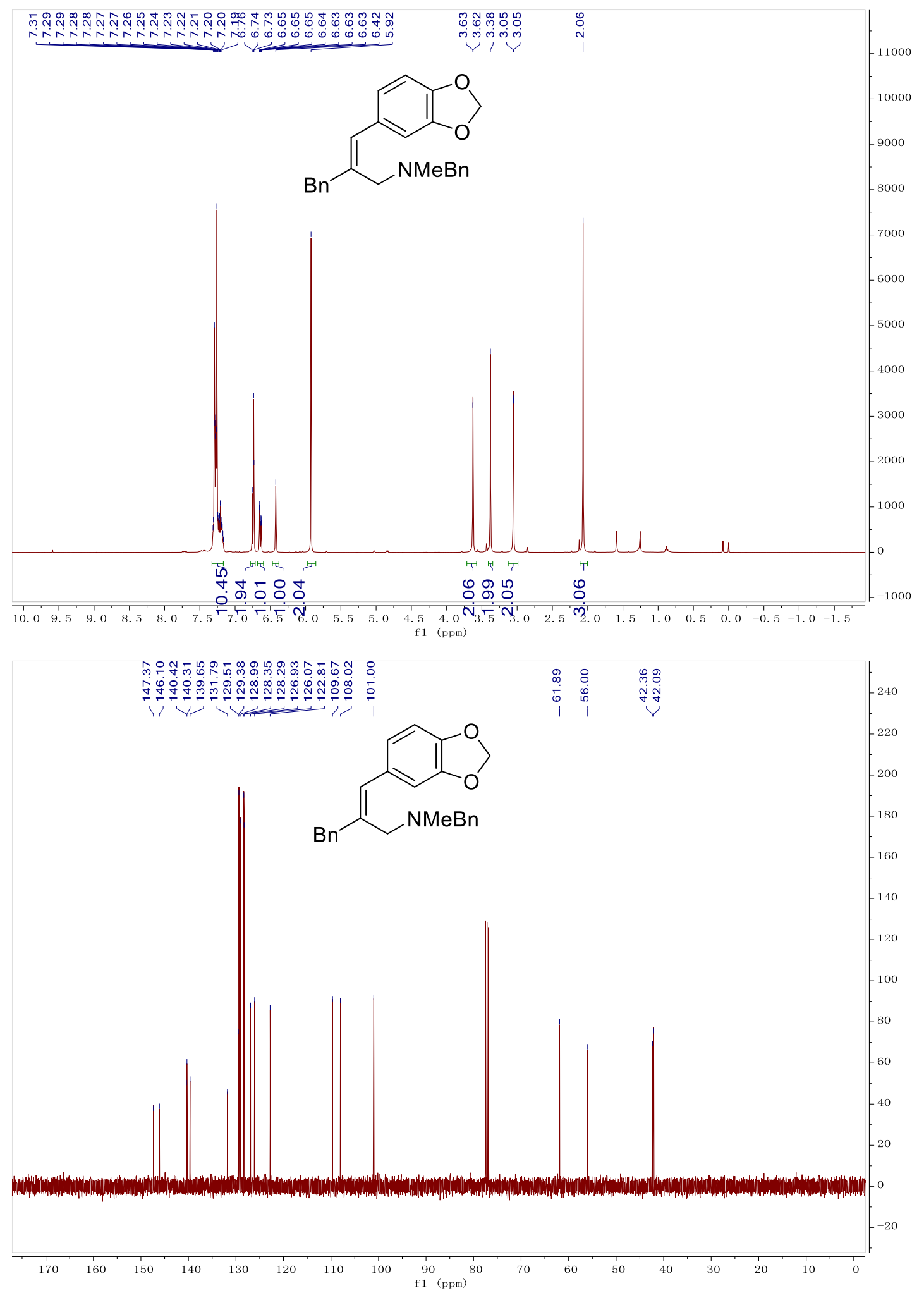




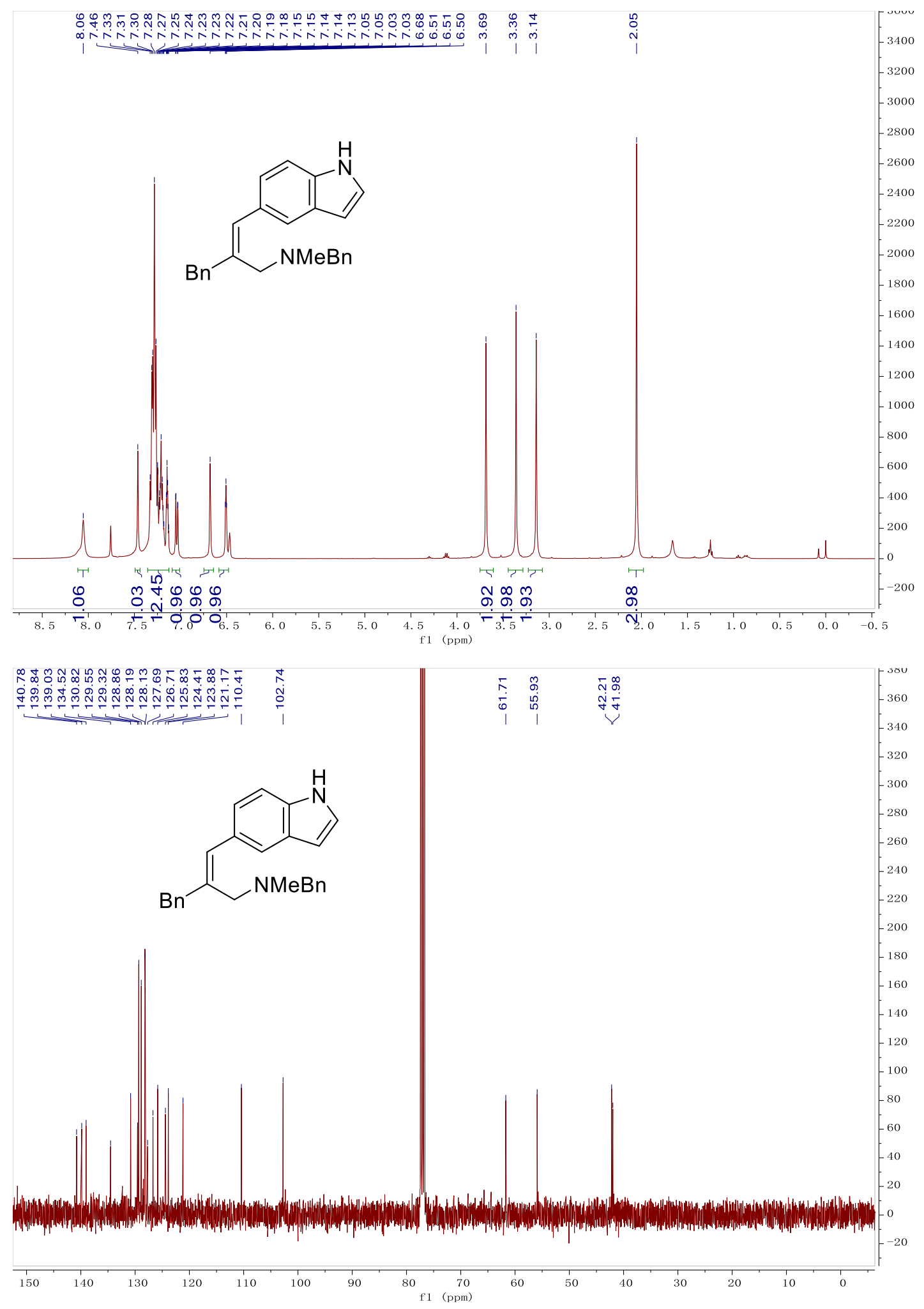



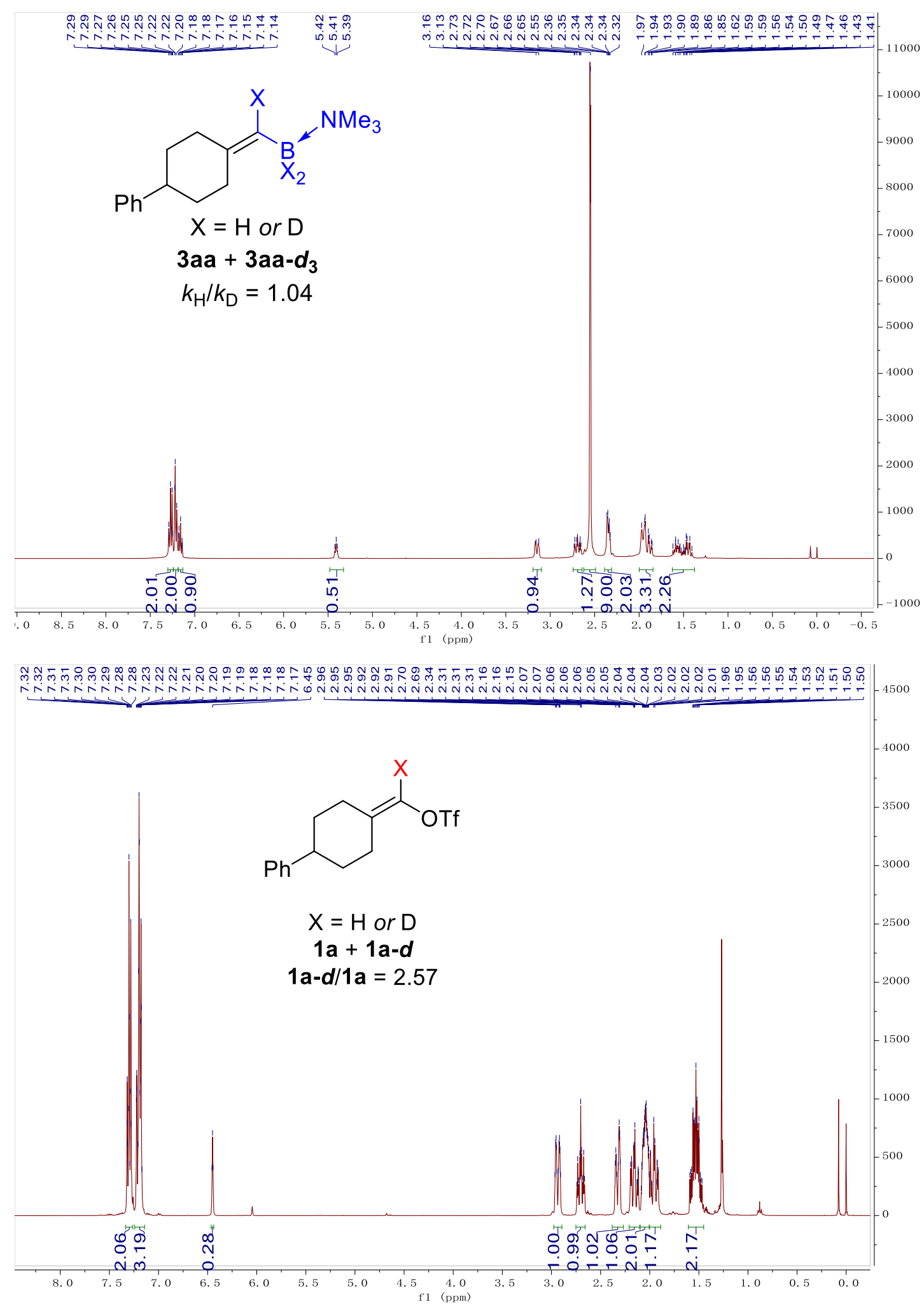


\section{References}

1 Armarego W. L. F.; Chai, C. L. L. Purification of Laboratory Chemicals-Six Edition. Elsevier Inc.: London, 2009.

${ }^{2}$ (a) Nainan, K. C.; Ryschkewitsch, G. E. A New Synthesis of Amine- and PhosphineBoranes. Inorg. Chem. 1969, 8, 2671-2674. (b) Hurtado, M.; Yánez, M.; Herrero, R.; Guerrero, A.; Dávalos, J. Z.; Abboud, J.-L. M.; Khater, B.; Guillemin, J.-C. The EverSurprising Chemistry of Boron: Enhanced Acidity of Phosphine · Boranes. Chem. Eur. J. 2009, 15, 4622-4629. (c) Gardner, S.; Kawamoto, T.; Curran, D. P. Synthesis of 1,3-Dialkylimidazol-2-ylidene Boranes from 1,3-Dialkylimidazolium Iodides and Sodium Borohydride. J. Org. Chem. 2015, 80, 9794-9797.

3 Stang, P. J.; Treptow, W. Single-Step Improved Synthesis of Primary and Other Vinyl Trifluoromethanesulfonates. Synthesis, 1980, 4, 283-284.

${ }^{4}$ Engesser, T.; Brückner, R. Synthesis of trans-Configured Enol Ethers by a Sequence of syn-Selective Glycolate Aldol Addition, Hydrolysis, and Grob Fragmentation. Eur. J. Org. Chem. 2017, 5789-5794.

5 a) Wang, C.; Tobrman, T.; Xu, Z.-Q.; Negishi, E. Highly Regio- and Stereoselective Synthesis of (Z)-Trisubstituted Alkenes via Propyne Bromoboration and Tandem PdCatalyzed Cross-Coupling. Org. Lett. 2009, 11, 4092-4095. b) Chen, Jack. L.-Y.; Aggarwal, V. K. Highly Diastereoselective and Enantiospecific Allylation of Ketones and Imines Using Borinic Esters: Contiguous Quaternary Stereogenic Centers. Angew. Chem. Int. Ed. 2014, 53, 10992 -10996. c) Zhurakovskyi, O.; Dias, R. M. P.; Noble, A.; Aggarwal, V. K. Stereo- and Regiocontrolled Methylboration of Terminal Alkynes. Org. Lett. 2018, 20, 3136-3139.

${ }^{6}$ Nakamura, S.; Aoki, T.; Ogura, T.; Wang, L.-B.; Toru, T. Highly Enantioselective Reaction of a-Selenoorganolithium Compounds with Chiral Bis(oxazoline)s and Preparation of Enantioenriched Benzylidencyclohexanes. J. Org. Chem. 2004, 69, 8916-8923.

7 Uenishi, J.; Kawahama, R.; Yonemitsu, O.; Tsuji, J. Stereoselective Hydrogenolysis of 1,1-Dibromo-1-alkenes and Stereospecific Synthesis of Conjugated (Z)-Alkenyl Compounds. J. Org. Chem., 1998, 63, 8965-8975.

8 a) Becke, A. D. A New Mixing of Hartree-Fock and Local Density-Functional 
Theories. J. Chem. Phys. 1993, 98, 1372-1377. b) Becke, A. D. Density-Functional Thermochemistry. III. The Role of Exact Exchange. J. Chem. Phys. 1993, 98, 56485652.

9 Hehre, W. J.; Ditchfeld, R.; Pople, J. A. Self-Consistent Molecular Orbital Ethods. XII. Further Extensions of Gaussian-Type Basis Sets for Use in Molecular Orbital Studies of Organic Molecules. J. Chem. Phys. 1972, 56, 2257-2261.

${ }^{10}$ a) Zhao, Y.; Truhlar, D. G. The M06 Suite of Density Functionals for Main Group hermochemistry, Thermochemical Kinetics, Noncovalent Interactions, Excited States, and Transition Elements. Theor. Chem. Acc. 2008, 120, 215-241. b) Frisch, M. J.; Pople, J. A.; Binkley, J. S. Self-Consistent Molecular Orbital Methods 25. Supplementary Functions for Gaussian Basis Sets. J. Chem. Phys. 1984, 80, 32653269.

11 Grimme, S.; Antony, J.; Ehrlich, S.; Krieg, H. A Consistent and Accurate ab Initio Parametrization of Density Functional Dispersion Correction (DFT-D) for the 94 Elements H-Pu. J. Chem. Phys. 2010, 132, 154104-154123.

12 Marenich, A. V.; Cramer, C. J.; Truhlar, D. G. Universal Solvation Model Based on Solute Electron Density and on a Continuum Model of the Solvent Defined by the Bulk Dielectric Constant and Atomic Surface Tensions. J. Phys. Chem. B, 2009, 113, 6378-6396.

${ }^{13}$ Legault, C. Y. CYLview, 1.0b. 2009, http://www.cylview.org.

${ }^{14}$ Pierens, J. K. ${ }^{1} \mathrm{H}$ and ${ }^{13} \mathrm{C}$ NMR Scaling Factors for the Calculation of Chemical Shifts in Commonly Used Solvents Using Density Functional Theory. J. Comput. Chem. 2014, 35, 1388-1394. 